\title{
EXPERIMENTAL AND NUMERICAL EVALUATION OF THE PERFORMANCES OF ELECTROMAGNETIC MINIATURE ENERGY HARVESTERS DRIVEN BY AIR FLOW
}

\author{
HAN NUOMIN \\ School of Mechanical and Aerospace Engineering
}

A thesis submitted to the Nanyang Technological University

in partial fulfillment of the requirements

for the degree of Doctor of Philosophy 


\section{Acknowledgement}

I would like to express my deepest gratitude to my PhD supervisor-Prof. Dan Zhao for the invaluable guidance, advice and lessons during my PhD study in Nanyang Technological University. I especially thank him for giving me freedom and support in pursuing my research interest in renewable and green energy.

My sincere appreciation also goes to all those who helped me during my $\mathrm{PhD}$ research. I would like to thank Prof WeiminHuang for lending me the MakerBot 3D Printer. I also would like to thank Mr. Zhou Ye who trained me about 3D Printer operation. Additionally, I would like to thank Mr. Cheo Hock Leong and Mr. Lam Kim Kheong for kindly giving me valuable suggestions and help. Without their rich experience in 3D printing, my research could not proceed so smoothly. I would like to thank Miss $\mathrm{Ng}$ Chai Chi for her help in conducting experimental study on conventional wind turbines. And I would also like to thank Dr. Arne Reinecke and Dr. Ernest Goh for their help and inspiring discussion on CFD simulations.

Last but not least, I would like to express my appreciation for the support and inspiring discussion received from my friends and families. 


\section{Table of contents}

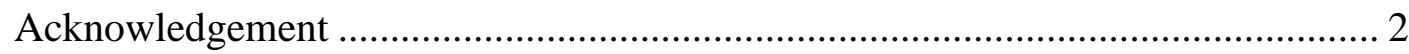

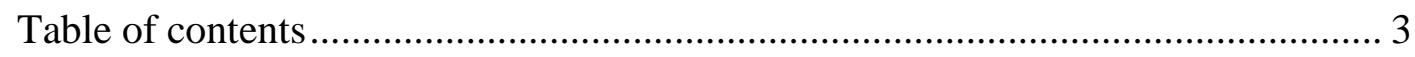

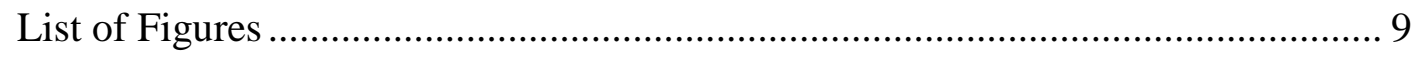

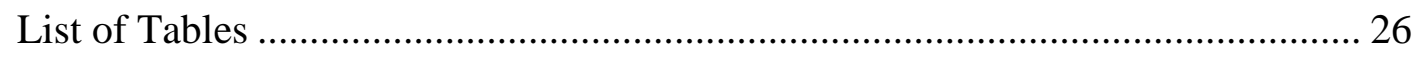

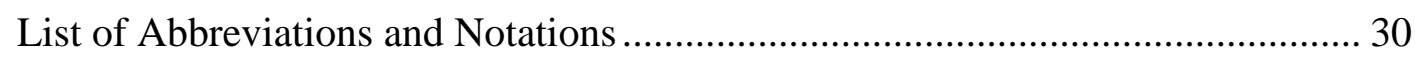

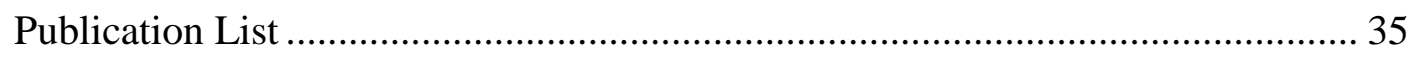

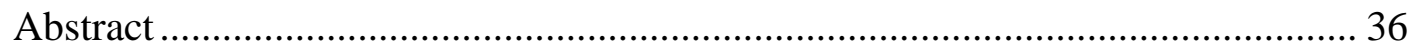

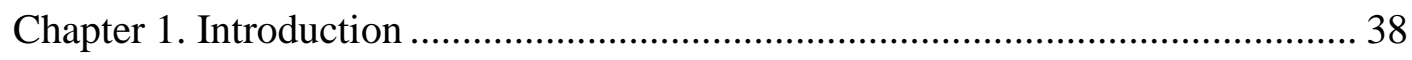

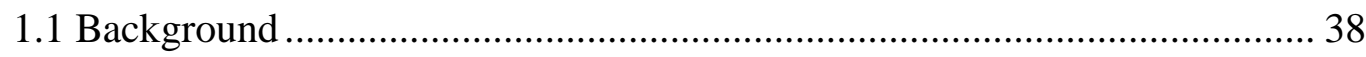

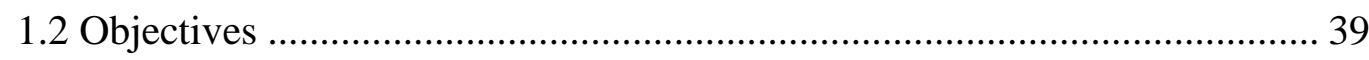

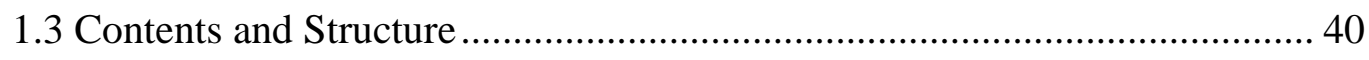

Chapter 2. Literature Review ......................................................................... 42

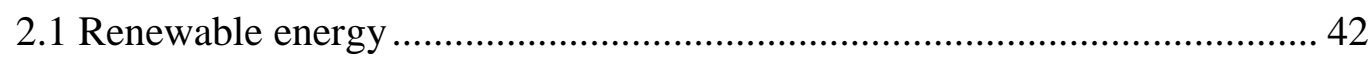

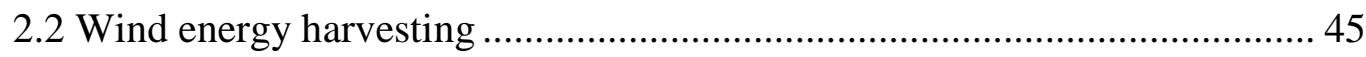

2.2.1 Wind turbine aerodynamics ................................................................ 46

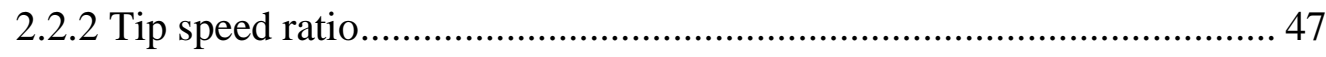

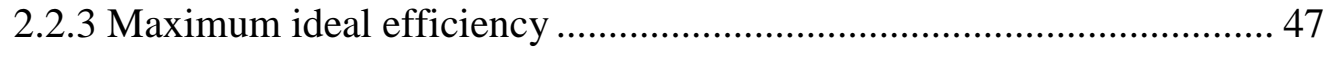

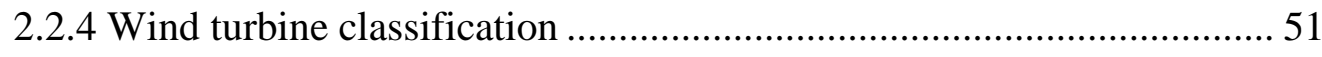


2.2.5 Urban wind energy

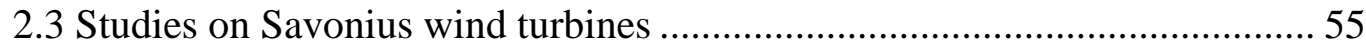

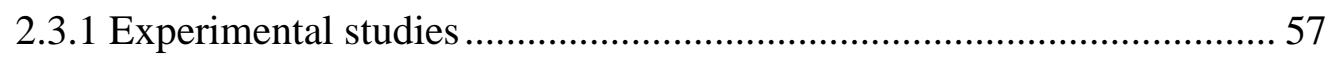

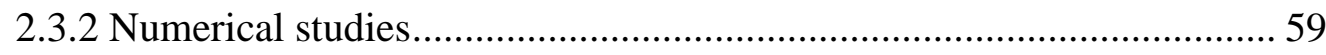

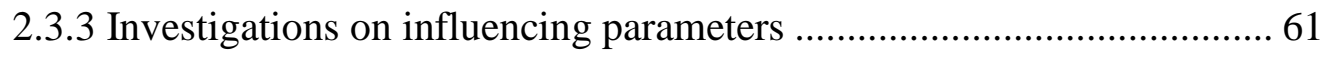

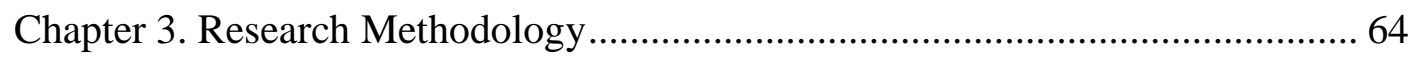

3.1 Energy harvester performance evaluation and hypothesis.............................. 64

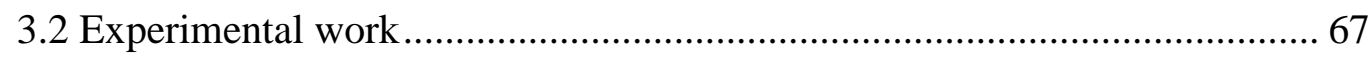

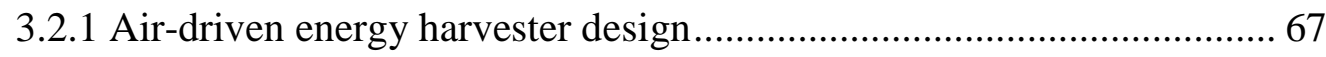

3.2.2 Air-driven energy harvester auxiliary parts design .............................. 75

3.2.3 Fabrication of air-driven energy harvesters and auxiliary parts ............ 78

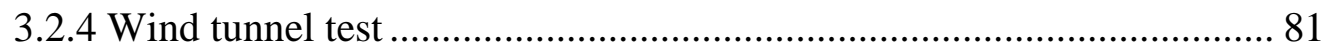

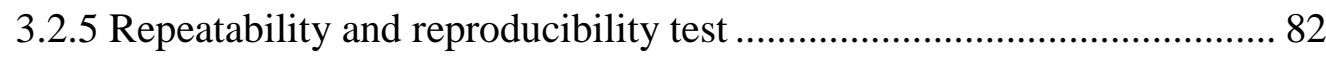

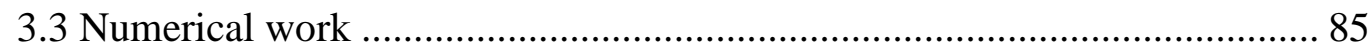

3.3.1 Numerical model description ................................................................ 85

3.3.2 Static performance of air-driven energy harvester................................ 96

3.3.3 Dynamic performance of air-driven energy harvesters.......................... 97

Chapter 4. Results and Discussion..................................................................... 99

4.1 Preliminary parametric measurements ........................................................ 99 
4.1.1 Performance of the benchmark model

4.1.2 Effect of the blade number $\boldsymbol{N}$

112

4.1.3 Effect of the energy harvester geometric size $(\boldsymbol{S R})$

4.1.4 Effect of the energy harvester aspect ratio $(\boldsymbol{A R})$.

129

4.1.5 Effect of types of the energy harvester central part ........................ 136

4.1.6 Effect of energy harvester end plates ......................................... 142

4.1.7 Effect of the energy harvester orientation..................................... 147

4.18 Concluding remarks ............................................................... 151

4.2 Effect of the harvester orientation....................................................... 153

4.2.1 Combined effect of energy harvester orientation and number of blades

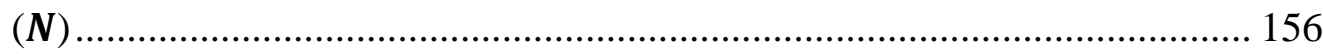

4.2.2 Combined effect of energy harvester orientation and geometric size $(\boldsymbol{S} \boldsymbol{R})$ 161

4.2.3 Combined effect of energy harvester orientation and aspect ratio (AR) 166

4.2.4 Combined effect of energy harvester orientation and central part....... 170

4.2.5 Combined effect of energy harvester orientation and end plates........ 172

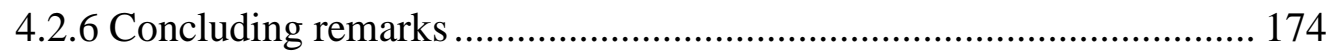

4.3 Study on blade shape profiles ...................................................... 178

4.3.1 Energy harvester with cross-sectional profile shape $\mathrm{S} 2550$.............. 181 
4.3.2 Energy harvester with cross-sectional profile shape S7550

4.3.3 Energy harvester with cross-sectional profile shape S5025 ................ 186

4.3.4 Energy harvester with cross-sectional profile shape S2525 ................ 188

4.3.5 Energy harvester with cross-sectional profile shape S7525 ................ 190

4.3.6 Energy harvester with cross-sectional profile shape D2550-7550 „..... 193

4.3.7 Energy harvester with cross-sectional profile shape D2525-7525 ...... 195

4.3.8 Concluding remarks ........................................................................... 197

4.4 Comparison with other air-driven energy harvesters.................................... 199

4.4.1 Comparison with miniature horizontal axis wind turbine..................... 199

4.4.2 Comparison with other air-driven harvesters reported in literature..... 201

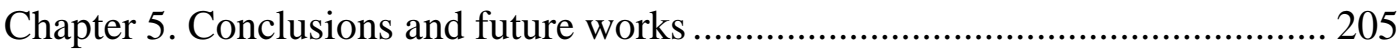

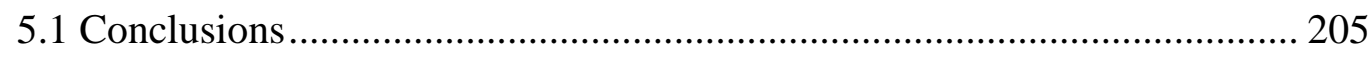

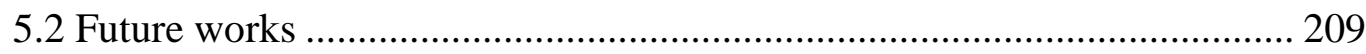

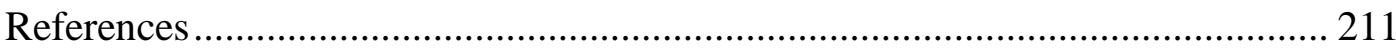

Appendix A: Energy harvester model design .................................................... 225

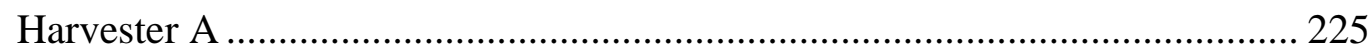

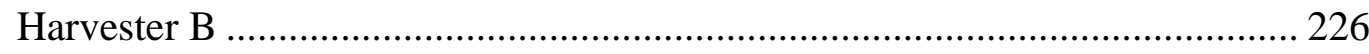

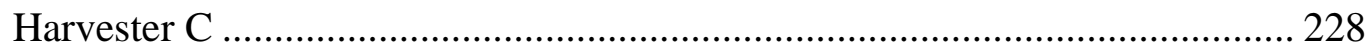

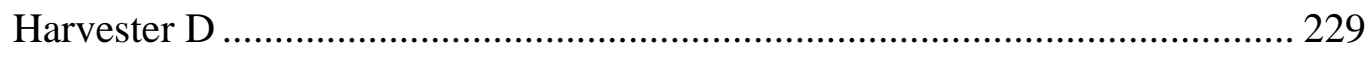


Harvester E.................................................................................... 231

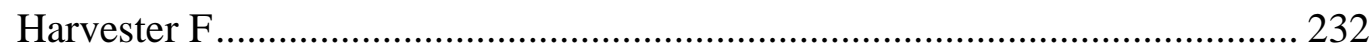

Harvester G .................................................................................... 234

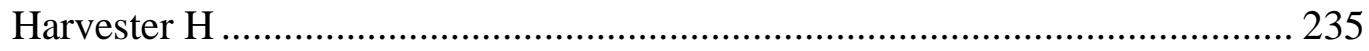

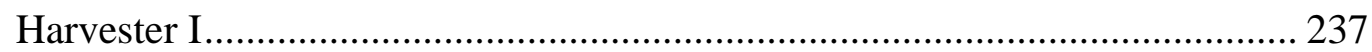

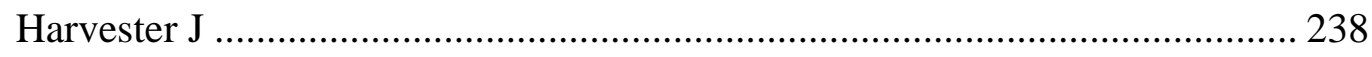

Appendix B: Wind tunnel results for preliminary parametric study ................... 240

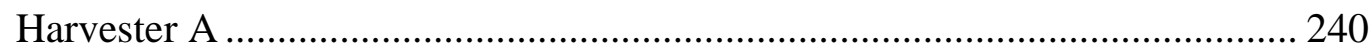

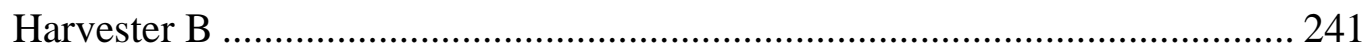

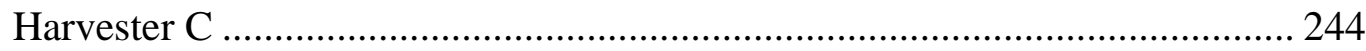

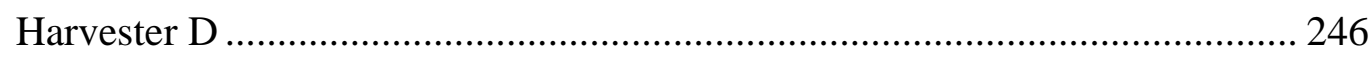

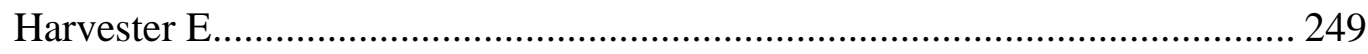

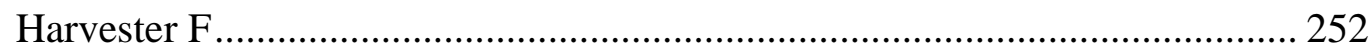

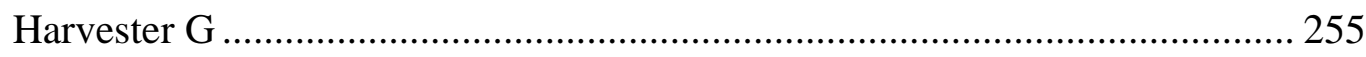

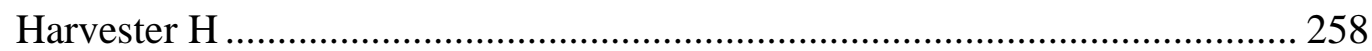

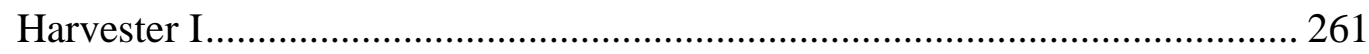

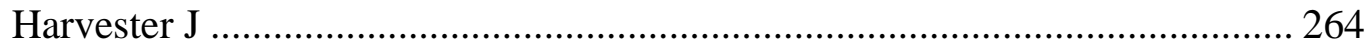

Appendix C: Wind tunnel results for harvester orientation case study ............... 269

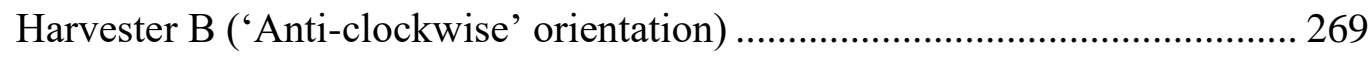




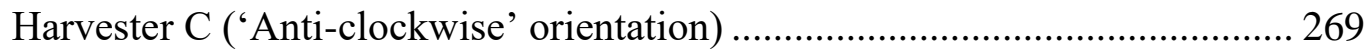

Harvester D ('Anti-clockwise' orientation) ................................................ 270

Harvester E ('Anti-clockwise' orientation) ............................................... 270

Harvester F ('Anti-clockwise' orientation).............................................. 271

Harvester G ('Anti-clockwise’ orientation) ............................................... 272

Harvester H ('Anti-clockwise' orientation) .............................................. 272

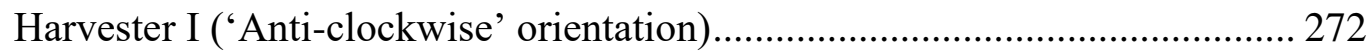




\section{List of Figures}

Fig.1 Estimated renewable energy share of global electricity production, end-2014.

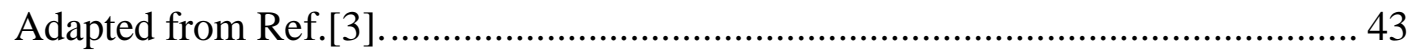

Fig.2(a) Global annual installed wind capacity 2000-2015. (b) Global cumulative installed wind capacity 2000-2015. Adapted from Ref.[6]................................ 44

Fig.3 Global cumulative wind power capacity projection (GW). Adapted from

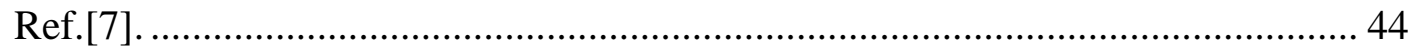

Fig.4 Control volume for actuator disk model of wind turbine. V is air velocity; 1,

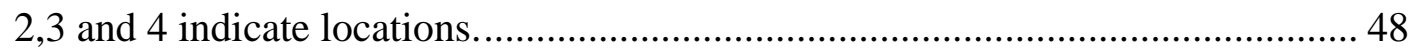

Fig.5 The power coefficient as a function of tip speed ratio for different wind turbine machines designs. Adapted from Ref. [13]. Note that in some previous publications (Ref. [12]), the efficiency curves of the American multiblade and the Savonius designs were inadvertently switched........................................... 50

Fig.6 Specific wind speeds in relation to wind turbine operation. Adapted from Ref. [16] 54

Fig.7 Schematic diagram of the experimental set-up for Savonius rotor testing built

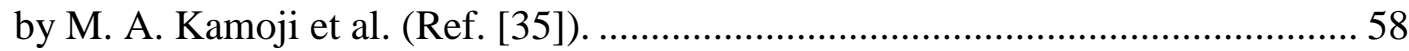

Fig.8 Views of helical Savonius rotors with various shapes and sizes of end plates

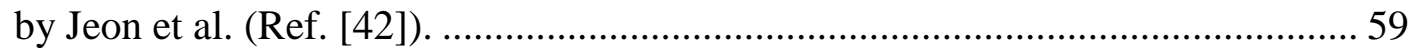

Fig.9 Illustration of air-driven energy harvesting system............................. 69 
Fig.10 Orientation of (a) Model A (Lower/clockwise orientation) and (b) Model J

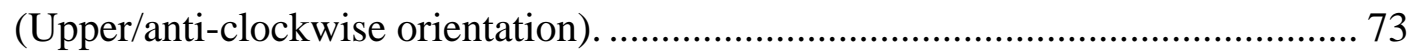

Fig.11 (a) Air-driven energy harvester support and (b) induction coil holder....... 75

Fig.12 Schematic diagram illustrating air-driven energy harvesting system

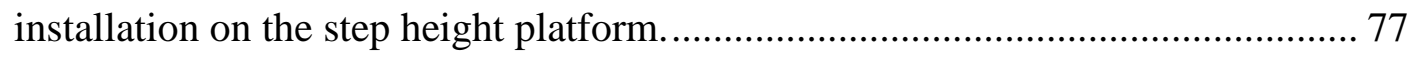

Fig.13 Energy harvesting system and step height platform installed in the wind

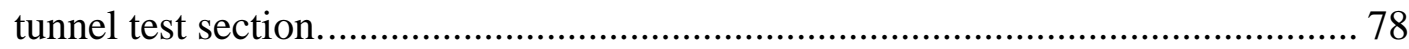

Fig.14 Classification of 3DP techniques. Adapted from Ref. [86]...................... 79

Fig.15 (a) Software preparing printer instruction file and (b) MakerBot Replicator 2

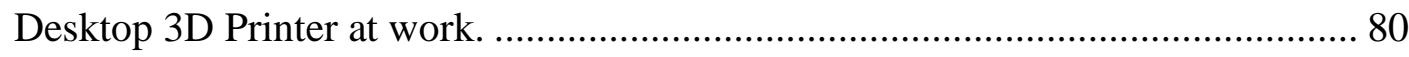

Fig.16 Closed loop wind tunnel at Nanyang Technological University............... 81

Fig.17 Comparison of measured results at different runs............................... 83

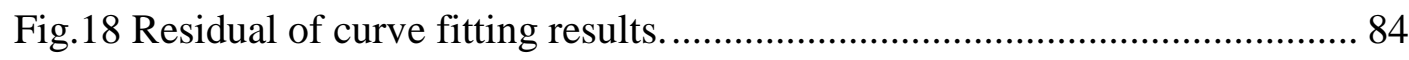

Fig.19 Relative errors in experimentally measured results.............................. 84

Fig.20 Side and top views of computational domain.................................... 88

Fig.21 View of meshed situation for (a) whole computational domain and (b) closer view of 'Rotor sub-domain' containing the energy harvester..................... 90

Fig.22 Variation of torque value with different levels of mesh fineness. ('num' denotes numerically predicted results and 'exp' denotes experimental results).... 92 Fig.23 Validation of numerical study results. (Specification of Model 1 and 2 can be found in Table 8.Pair of empty markers from numerical and experimental results 
share the same conditions. 'num' denotes numerically predicted results and 'exp'

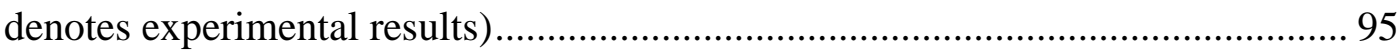

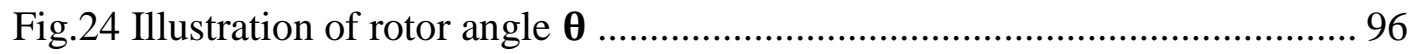

Fig.25 Time evolution of measured emf from harvester A at various Reynolds

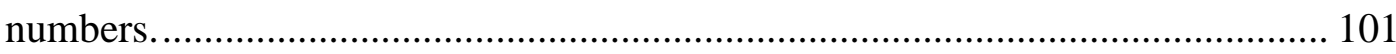

Fig.26 FFT plot of measured emf from harvester Model A at various Reynolds

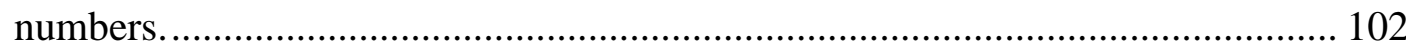

Fig.27 Phase plot of measured emf from harvester Model A at various Reynolds numbers. 103

Fig.28 (a) RPM variation with Reynolds number. (b) Electrical power output varied with RPM. (c) Electrical power and (d) Overall energy conversion efficiency varied with Reynolds number for energy harvester Model A. (c-f denotes curve fitting and e denotes experimental results). 104

Fig.29 Red and white LED lights powered by the energy harvester A. 106

Fig.30 Variation of static torque coefficients at different rotor angles at different Reynolds numbers for harvester A. 107

Fig.31 Pressure distribution patterns at $\operatorname{Re}=0.99 \times 10^{5}$ when (a) $\theta=40^{\circ}$, (b) $\theta=100^{\circ}$ and at $\operatorname{Re}=2.97 \times 10^{5}$ when (c) $\theta=40^{\circ}$, (d) $\theta=100^{\circ}$ for energy harvester A.......... 108 Fig.32 Performance characteristic curves for harvester $A$ at $\operatorname{Re}=1.49 \times 10^{5}$, $\operatorname{Re}=2.97 \times 10^{5}$ and $\operatorname{Re}=4.46 \times 10^{5}$ 109 
Fig.33Pressure distribution around energy harvester $\mathrm{A}$ at (a) $\lambda=0.167$, $\operatorname{Re}=1.49 \times 10^{5}$, (b) $\lambda=1.0, \operatorname{Re}=1.49 \times 10^{5}$ and (c) $\lambda=0.167, \operatorname{Re}=2.97 \times 10^{5}$.

Fig.34 Instantaneous dynamic torque coefficient at (a) $\operatorname{Re}=1.49 \times 10^{5}:$ at $\lambda=1.0$ the highest possible power coefficient is achieved and at $\lambda=0.167$ the rotation speed is set to be the same in experiments. And at (b) $\operatorname{Re}=2.97 \times 10^{5}$ : at $\lambda=1.25$ the highest possible power coefficient is achieved and at $\lambda=0.5$ the rotation speed is set to be the same in experiments.

Fig.35 (a) RPM variation with Reynolds number. (b) Electrical power output varied with RPM. (c) Electrical power and (d) Overall energy conversion efficiency varied with Reynolds number for energy harvester B. (c-f denotes curve fitting and e denotes experimental results). 114

Fig.36 (a) RPM variation with Reynolds number. (b) Electrical power output varied with RPM. (c) Electrical power and (d) Overall energy conversion efficiency varied with Reynolds number for energy harvester C. (c-f denotes curve fitting and e denotes experimental results).

Fig.37 Comparison of overall energy conversion efficiency from harvester A, B and $\mathrm{C}$ with different blade numbers, as the Reynolds number is increased. (c- $\mathrm{f}$ denotes curve fitting and e denotes experimental results).

Fig.38 Variation of static torque coefficients at different rotor angles at different Reynolds numbers for harvester B 118

Fig.39 Variation of static torque coefficients at different rotor angles at different Reynolds numbers for harvester C 
Fig.40 Performance characteristic curves for harvester $B$ at $\operatorname{Re}=4.46 \times 10^{5}$. (The blue line indicates values attained by numerical work and the red line indicates experimentally achieved tip speed ratio).

Fig.41 Performance characteristic curves for harvester $C$ at $R e=4.46 \times 10^{5}$. (The blue line indicates values attained by numerical work and the red line indicates experimentally achieved tip speed ratio).

Fig.42 (a) RPM variation with Reynolds number. (b) Electrical power output varied with RPM. (c) Electrical power and (d) Overall energy conversion efficiency varied with Reynolds number for energy harvester D. (c-f denotes curve fitting and e denotes experimental results)....

Fig.43 (a) RPM variation with Reynolds number. (b) Electrical power output varied with RPM. (c) Electrical power and (d) Overall energy conversion efficiency varied with Reynolds number for energy harvester E. (c-f denotes curve fitting and e denotes experimental results). 123

Fig.44 Comparison of overall energy conversion efficiency from harvester A, D and E with different geometric sizes, as the Reynolds number is increased. (c-f denotes curve fitting and e denotes experimental results).

Fig.45 Variation of static torque coefficients at different rotor angles at different Reynolds numbers for harvester $\mathrm{D}$. 125

Fig.46 Variation of static torque coefficients at different rotor angles at different Reynolds numbers for harvester E. 126 
Fig.47 Performance characteristic curves for harvester $D$ at $R e=4.46 \times 10^{5}$. (The blue line indicates values attained by numerical work and the red line indicates experimentally achieved tip speed ratio).

Fig.48 Performance characteristic curves for harvester $E$ at $R e=4.46 \times 10^{5}$. (The blue line indicates values attained by numerical work and the red line indicates experimentally achieved tip speed ratio).

Fig.49 (a) RPM variation with Reynolds number. (b) Electrical power output varied with RPM. (c) Electrical power and (d) Overall energy conversion efficiency varied with Reynolds number for energy harvester F. (c-f denotes curve fitting and e denotes experimental results). 129

Fig.50 (a) RPM variation with Reynolds number. (b) Electrical power output varied with RPM. (c) Electrical power and (d) Overall energy conversion efficiency varied with Reynolds number for energy harvester G. (c-f denotes curve fitting and e denotes experimental results).

Fig.51 Comparison of overall energy conversion efficiency from harvester A, F and $\mathrm{G}$ with different aspect ratios, as the Reynolds number is increased. (c- $\mathrm{f}$ denotes curve fitting and e denotes experimental results).

Fig.52 Variation of static torque coefficients at different rotor angles at different Reynolds numbers for harvester F.

Fig.53 Variation of static torque coefficients at different rotor angles at different Reynolds numbers for harvester $\mathrm{G}$. 134 
Fig.54 Performance characteristic curves for harvester $F$ at $R e=4.46 \times 10^{5}$. (The blue line indicates values attained by numerical work and the red line indicates experimentally achieved tip speed ratio). 135

Fig.55 Performance characteristic curves for harvester $G$ at $R e=4.46 \times 10^{5}$. (The blue line indicates values attained by numerical work and the red line indicates experimentally achieved tip speed ratio). 136

Fig.56 (a) RPM variation with Reynolds number. (b) Electrical power output varied with RPM. (c) Electrical power and (d) Overall energy conversion efficiency varied with Reynolds number for energy harvester H. (c-f denotes curve fitting and e denotes experimental results) 138

Fig.57 Comparison of overall energy conversion efficiency from harvester A and $\mathrm{H}$ with different types of central part, as the Reynolds number is increased. (c-f denotes curve fitting and e denotes experimental results).

Fig.58 Variation of static torque coefficients at different rotor angles at different Reynolds numbers for harvester $\mathrm{H}$. 140

Fig.59 Pressure distribution at minimum static torque coefficient value for energy harvester $\mathrm{H}$ 141

Fig.60 Performance characteristic curves for harvester $H$ at $R e=4.46 \times 10^{5}$. (The blue line indicates values attained by numerical work and the red line indicates experimentally achieved tip speed ratio).

Fig.61 (a) RPM variation with Reynolds number. (b) Electrical power output varied with RPM. (c) Electrical power and (d) Overall energy conversion 
efficiency varied with Reynolds number for energy harvester I. (c-f denotes curve fitting and e denotes experimental results). 142

Fig.62 Comparison of overall energy conversion efficiency from harvester A and I with or without end plates, as the Reynolds number is increased. (c-f denotes curve fitting and e denotes experimental results).

Fig.63 Variation of static torque coefficients at different rotor angles at different Reynolds numbers for harvester I. 144

Fig.64 Pressure distribution at minimum static torque coefficient value for energy harvester I. 145

Fig.65 Performance characteristic curves for harvester I at $\mathrm{Re}=4.46 \times 10^{5}$. (The blue line indicates values attained by numerical work and the red line indicates experimentally achieved tip speed ratio).

Fig.66 (a) RPM variation with Reynolds number. (b) Electrical power output varied with RPM. (c) Electrical power and (d) Overall energy conversion efficiency varied with Reynolds number for energy harvester J. (c-f denotes curve fitting and e denotes experimental results). 148

Fig.67 Comparison of overall energy conversion efficiency from harvester A and J installed in different orientations, as the Reynolds number is increased. (c-f denotes curve fitting and e denotes experimental results).

Fig.68 Variation of static torque coefficients at different rotor angles at different Reynolds numbers for harvester $\mathrm{J}$ 150 
Fig.69 Performance characteristic curves for harvester $\mathrm{J}$ at $\mathrm{Re}=4.46 \times 10^{5}$. (The blue line indicates values attained by numerical work and the red line indicates experimentally achieved tip speed ratio).

Fig.70 Pressure distribution and velocity vector diagram of benchmark energy harvester model with (a) 'clockwise' orientation and (b) 'anti-clockwise' orientation.

Fig.71 Variation of RPM with Re for 'clockwise'(harvester A) and 'anticlockwise'(harvester J) installation orientation. ('exp' denotes experimental data and 'cf' denotes curve fitting line) 154

Fig.72 Pressure distribution at $R e=1.49 \times 10^{5}$ for benchmark energy harvester with 'clockwise' and 'anti-clockwise' installation orientation ..... 155

Fig.73 Variation of RPM with Re for (a) harvester B and (b) harvester C with 'clockwise' and 'anti-clockwise' installation orientation. ('exp' denotes experimental data and 'cf' denotes curve fitting line) 157

Fig.74 Instantaneous $\mathrm{C}_{\mathrm{Td}}$ within one revolution for energy harvester (a) B and (b) $\mathrm{C}$ at $\operatorname{Re}=1.49 \times 10^{5}$ and $\omega=5 \mathrm{rad} / \mathrm{s}$.

Fig.75 Pressure distribution at $R e=1.49 \times 10^{5}$ for energy harvester (a) B and (b) C with 'clockwise' and 'anti-clockwise' installation orientation. 160

Fig.76 Variation of RPM with Re for harvester (a) D and (b) E with 'clockwise' and 'anti-clockwise' installation orientation. ('exp' denotes experimental data and 'cf' denotes curve fitting line) 162 
Fig.77 Instantaneous $\mathrm{C}_{\mathrm{Td}}$ within one revolution for energy harvester (a) D and (b) E at $\operatorname{Re}=1.49 \times 10^{5}$ and $\omega=5 \mathrm{rad} / \mathrm{s}$

Fig.78 Pressure distribution at $R e=1.49 \times 10^{5}$ for energy harvester (a) D and (b) E with 'clockwise' and 'anti-clockwise' installation orientation. 164

Fig.79 Variation of RPM with Re for harvester (a) F and (b) G with 'clockwise' and 'anti-clockwise' installation orientation. ('exp' denotes experimental data and 'cf' denotes curve fitting line). 166

Fig.80 Instantaneous $C_{\text {Td }}$ within one revolution for energy harvester (a) $F$ and (b) $G$ at $\operatorname{Re}=1.49 \times 10^{5}$ and $\omega=5 \mathrm{rad} / \mathrm{s}$ 168

Fig.81Pressure distribution at $R e=1.49 \times 10^{5}$ for energy harvester (a) $F$ and (b) G with 'clockwise' and 'anti-clockwise' installation orientation. 169

Fig.82 Variation of RPM with Re for harvester $H$ with 'clockwise' and 'anticlockwise' installation orientation. ('exp' denotes experimental data and 'cf' denotes curve fitting line) 170

Fig.83 Instantaneous $\mathrm{C}_{\mathrm{Td}}$ within one revolution for energy harvester $\mathrm{H}$ at $\mathrm{Re}=1.49 \times 10^{5}$ and $\omega=5 \mathrm{rad} / \mathrm{s}$ 171

Fig.84 Pressure distribution at $R e=1.49 \times 10^{5}$ for energy harvester H with 'clockwise' and 'anti-clockwise' installation orientation. 171

Fig.85 Variation of RPM with Re for harvester I with 'clockwise' and 'anticlockwise' installation orientation. ('exp' denotes experimental data and 'cf' denotes curve fitting line) 172 
Fig.86 Instantaneous $\mathrm{C}_{\mathrm{Td}}$ within one revolution for energy harvester $\mathrm{I}$ at $\mathrm{Re}=1.49 \times 10^{5}$ and $\omega=5 \mathrm{rad} / \mathrm{s}$ 173

Fig.87 Pressure distribution at $R e=1.49 \times 10^{5}$ for energy harvester I with 'clockwise' and 'anti-clockwise' installation orientation. 174

Fig.88 Velocity variation in the domain of wind tunnel test section. 178

Fig.89 Blade cross-sectional profile design of S5050 model (harvester J). 179

Fig.90 Variation of static torque coefficients at different rotor angles at $\mathrm{Re}=1.98 \times 10^{5}$ forenergy harvester $\mathrm{J}$

Fig.91 Performance characteristic curves for energy harvester J, at $\operatorname{Re}=1.49 \times 10^{5}$. (c-f denotes curve fitting and $n$ denotes numerical results).

Fig.92 Blade cross-sectional profile design of S2550 model 181

Fig.93 Variation of static torque coefficients at different rotor angles at $\mathrm{Re}=1.98 \times 10^{5}$ for energy harvester with $\mathrm{S} 2550$ cross sectional profile shape 182

Fig.94Performance characteristic curves for energy harvester with S2550 cross sectional profile shape, at $\operatorname{Re}=1.49 \times 10^{5}$. (c-f denotes curve fitting and $\mathrm{n}$ denotes numerical results) 183

Fig.95 Blade cross-sectional profile design of S7550 model 183

Fig.96 Variation of static torque coefficients at different rotor angles at $\mathrm{Re}=1.98 \times 10^{5}$ for energy harvester with $\mathrm{S} 7550$ cross sectional profile shape 184 
Fig.97 Performance characteristic curves for energy harvester with S7550 cross sectional profile shape, at $\operatorname{Re}=1.49 \times 10^{5}$. (c-f denotes curve fitting and $\mathrm{n}$ denotes numerical results) 185

Fig.98 Blade cross-sectional profile design of S5025 model 186

Fig.99Variation of static torque coefficients at different rotor angles at $\mathrm{Re}=1.98 \times 10^{5}$ for energy harvester with $\mathrm{S} 5025$ cross sectional profile shape....... 187

Fig.100Performance characteristic curves for energy harvester with S5025 cross sectional profile shape, at $\mathrm{Re}=1.49 \times 10^{5}$. (c-f denotes curve fitting and $\mathrm{n}$ denotes numerical results) 187

Fig.101 Blade cross-sectional profile design of S2525 model 188

Fig.102 Variation of static torque coefficients at different rotor angles at $\mathrm{Re}=1.98 \times 10^{5}$ for energy harvester with $\mathrm{S} 2525$ cross sectional profile shape....... 189

Fig.103Performance characteristic curves for energy harvester with S2525 cross sectional profile shape, at $\mathrm{Re}=1.49 \times 10^{5}$. (c-f denotes curve fitting and $\mathrm{n}$ denotes numerical results) 190

Fig.104 Blade cross-sectional profile design of S7525 model 190

Fig.105 Variation of static torque coefficients at different rotor angles at $\mathrm{Re}=1.98 \times 10^{5}$ for energy harvester with $\mathrm{S} 7525$ cross sectional profile shape....... 191

Fig.106 Performance characteristic curves for energy harvester with S7525 cross sectional profile shape, at $\mathrm{Re}=1.49 \times 10^{5}$. (c-f denotes curve fitting and $\mathrm{n}$ denotes numerical results) 192 
Fig.107 Blade cross-sectional profile design of D2550-7550 model 193

Fig.108 Variation of static torque coefficients at different rotor angles at $\operatorname{Re}=1.98 \times 10^{5}$ for energy harvester with D2550-7550 cross sectional profile shape.

194

Fig.109 Performance characteristic curves for energy harvester with D2550-7550 cross sectional profile shape, at $R e=1.49 \times 10^{5}$. (c-f denotes curve fitting and $n$ denotes numerical results).

Fig.110 Blade cross-sectional profile design of D2525-7525 model 195

Fig.111Variation of static torque coefficients at different rotor angles at $\operatorname{Re}=1.98 \times 10^{5}$ for energy harvester with D2525-7525 cross sectional profile shape. 196

Fig.112 Performance characteristic curves for energy harvester with D2525-7525 cross sectional profile shape, at $R e=1.49 \times 10^{5}$. (c-f denotes curve fitting and $n$ denotes numerical results) 196

Fig.113 Two-blade and three-blade horizontal axis wind energy harvesters, both with diameter of 7 inch. 199

Fig.114 Overall energy conversion efficiencies varied with Reynolds number for 3blade HAWH with different swept area diameters. (c-f denotes curve fitting and e

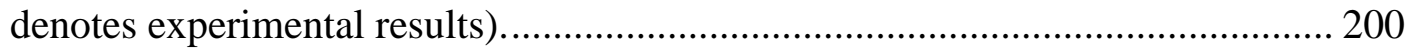


Figure115 Overall energy conversion efficiencies varied with Reynolds number for 3-blade HAWH with different swept area diameters. (c-f denotes curve fitting and e

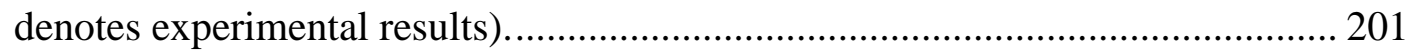

Fig.116 Comparison of maximum power density of other reported different scales energy harvesters. EWEA [91]. IEA [92]. Freere et al. [93]. Singh et al. [94].

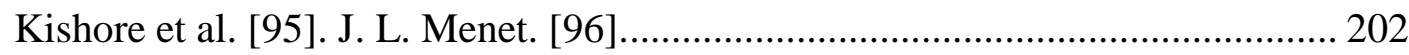

Fig.117Comparison of power coefficients of other reported energy harvesters at micro scale. Kamoji et al. [35]. Damak et al. [41]. Jeon et al. [42]. Nasef et al. [80]. Blackwell et al. [97]. Kacprazk et al. [98]. Mojola et al. [27] Saha et al (1) [33]. Saha et al (2) [34]. Sheldahl et al. (2-blade and 3-blade) [29]. Alexander and Holownia [30]. Baird and Pender [99]. Bergeless and Athanassiadis [100]. Sivasegaram and Sivapalan [101]. Bowden and Mc-Aleese [102]. Ogawa and Yoshida (without and with defector) [103]. Huda et al. (without and with defector)

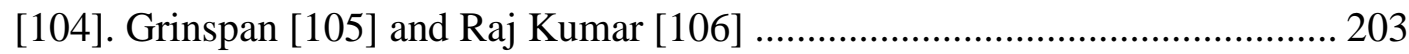

Fig.118 (a) 3D view, (b) Top view and (c) 3D printed model of harvester A...... 225

Fig.119 (a) 3D view, (b) Top view and (c) 3D printed model of harvester B ...... 227

Fig.120 (a) 3D view, (b) Top view and (c) 3D printed model of harvester C ...... 228

Fig.121 (a) 3D view, (b) Top view and (c) 3D printed model of harvester D ...... 230

Fig.122 (a) 3D view, (b) Top view and (c) 3D printed model of harvester E ...... 231

Fig.123 (a) 3D view, (b) Top view and (c) 3D printed model of harvester F....... 233

Fig.124 (a) 3D view, (b) Top view and (c) 3D printed model of harvester G..... 234 
Fig.125 (a) Comparison between harvester $\mathrm{H}$ and A, and (b) 3D printed model of

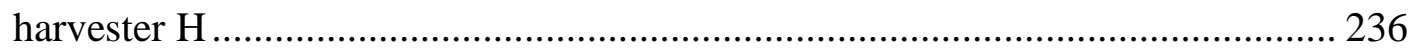

Fig.126 (a) 3D view, (b) Top view and (c) 3D printed model of harvester I ...... 237

Fig.127 Time evolution of measured emf from harvester B at various Reynolds

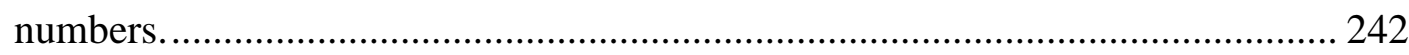

Fig.128 FFT plot of measured emf from harvester B at various Reynolds numbers. 243

Fig.129 Phase plot of measured emf from harvester B at various Reynolds numbers. 244

Fig.130 Time evolution of measured emf from harvester $\mathrm{C}$ at various Reynolds numbers 245

Fig.131 FFT plot of measured emf from harvester $\mathrm{C}$ at various Reynolds numbers. 246

Fig.132 Phase plot of measured emf from harvester $\mathrm{C}$ at various Reynolds numbers. 246

Fig.133 Time evolution of measured emf from harvester D at various Reynolds numbers. 247

Fig.134 FFT plot of measured emf from harvester D at various Reynolds numbers. 248

Fig.135 Phase plot of measured emf from harvester D at various Reynolds numbers. 
Fig.136 Time evolution of measured emf from harvester E at various Reynolds numbers. 250

Fig.137 FFT plot of measured emf from harvester E at various Reynolds numbers. 251

Fig.138 Phase plot of measured emf from harvester E at various Reynolds numbers.

Fig.139 Time evolution of measured emf from harvester $\mathrm{F}$ at various Reynolds numbers. 253

Fig.140 FFT plot of measured emf from harvester $\mathrm{F}$ at various Reynolds numbers. 254

Fig.141 Phase plot of measured emf from harvester F at various Reynolds numbers. 255

Fig.142 Time evolution of measured emf from harvester $\mathrm{G}$ at various Reynolds numbers. 256

Fig.143 FFT plot of measured emf from harvester $\mathrm{G}$ at various Reynolds numbers.

Fig.144 Phase plot of measured emf from harvester $\mathrm{G}$ at various Reynolds numbers.

Fig.145 Time evolution of measured emf from harvester $\mathrm{H}$ at various Reynolds

numbers. 259 
Fig.146 FFT plot of measured emf from harvester $\mathrm{H}$ at various Reynolds numbers. 260

Fig.147 Phase plot of measured emf from harvester $\mathrm{H}$ at various Reynolds numbers. 261

Fig.148 Time evolution of measured emf from harvester I at various Reynolds numbers. 262

Fig.149 FFT plot of measured emf from harvester I at various Reynolds numbers. 263

Fig.150 Phase plot of measured emf from harvester I at various Reynolds numbers. 264

Fig.151 Time evolution of measured emf from harvester $\mathrm{J}$ at various Reynolds numbers. 266

Fig.152 FFT plot of measured emf from harvester $\mathrm{J}$ at various Reynolds numbers. 267

Fig.153 Phase plot of measured emf from harvester $\mathrm{J}$ at various Reynolds numbers. 


\section{List of Tables}

Table 1 Urban wind energy utilization categories. Adapted from Ref. [16].......... 53

Table 2 Specifications of energy harvester models design in preliminary parametric study. 74

Table 3 Characteristic parameters of step height platform. 77

Table 4 Printer settings for energy harvester models and auxiliary parts fabrication.

Table 5 Frontal areas and corresponding blockage factors of energy harvesters investigated in preliminary parametric study 86

Table 6 ANSYS CFX settings for simulating the experiment............................... 88

Table 7 Details for meshes with different levels of fineness................................. 91

Table 8 Design parameters details of energy harvester models used for validation.

Table 9 Summary of performances of designed energy harvester models 152

Table 10 Summary of static performance of energy harvester models in different orientations. (For each orientation, first column is experimental cut-in Reynolds number and second column is range of numerically predicted static torque coefficient.) 174

Table 11Summary of dynamic performance of energy harvester models in different orientations. (For each orientation, first column is experimental overall energy 
conversion efficiency and second column is range of numerically predicted dynamic torque coefficient at $\omega=5 \mathrm{rad} / \mathrm{s}, R e=4.46 \times 10^{5}$. .) ................................ 176

Table 12 Summary of numerically studied static and dynamic performances of energy harvesters with different blade cross-sectional profile shapes, as compared with harvester J 198

Table 13 Design of harvester A 225

Table 14 Characteristic parameters of harvester A ............................................. 226

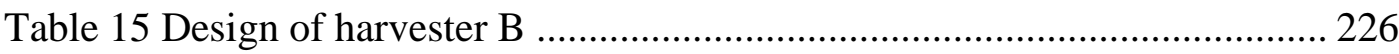

Table 16 Characteristic parameters of harvester B ……….................................... 227

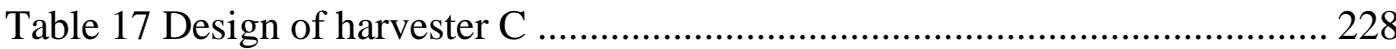

Table 18 Characteristic Parameters of harvester C ................................................. 229

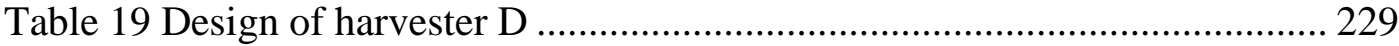

Table 20 Characteristic parameters of harvester D ........................................... 230

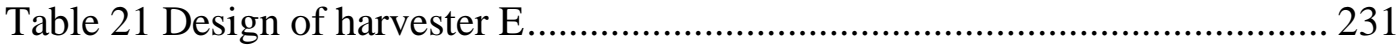

Table 22 Characteristic parameters of harvester E ............................................... 232

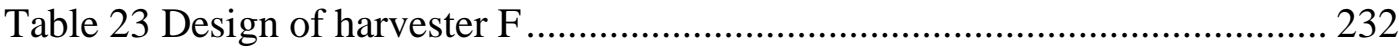

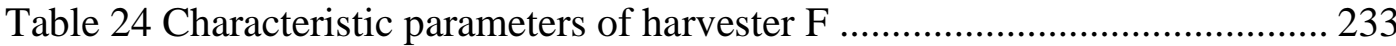

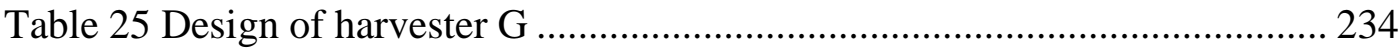

Table 26 Characteristic parameters of harvester G ............................................ 235

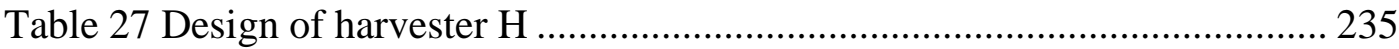


Table 28 Characteristic parameters of harvester $\mathrm{H}$ 236

Table 29 Design of harvester I 237

Table 30 Characteristic parameters of harvester I 238

Table 31 Design of harvester J. 238

Table 32 Characteristic parameters of harvester $\mathbf{J}$ 238

Table 33 Experimental measurements results of energy harvester A 240

Table 34 Experimental measurements results of energy harvester B 241

Table 35 Experimental measurements results of energy harvester C 244

Table 36 Experimental measurements results of energy harvester D 246

Table 37 Experimental measurements results of energy harvester E 249

Table 38 Experimental measurements results of energy harvester $\mathrm{F}$ 252

Table 39 Experimental measurements results of energy harvester $G$ 255

Table 40 Experimental measurements results of energy harvester $\mathrm{H}$ 258

Table 41 Experimental measurements results of energy harvester I 261

Table 42 Experimental measurements results of energy harvester $\mathbf{J}$ 264

Table 43 Experimental measurements results of energy harvester B in 'anticlockwise' orientation 269

Table 44 Experimental measurements results of energy harvester $\mathrm{C}$ in 'anticlockwise' orientation 269 
Table 45 Experimental measurements results of energy harvester D in 'anti-

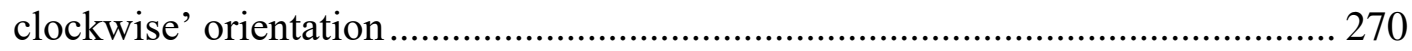

Table 46 Experimental measurements results of energy harvester $\mathrm{E}$ in 'anti-

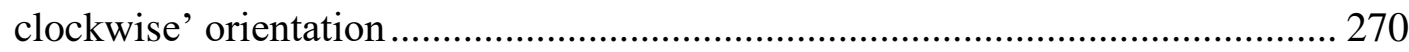

Table 47 Experimental measurements results of energy harvester $F$ in 'anticlockwise' orientation 271

Table 48 Experimental measurements results of energy harvester $G$ in 'anticlockwise' orientation 272

Table 49 Experimental measurements results of energy harvester $H$ in 'anti-

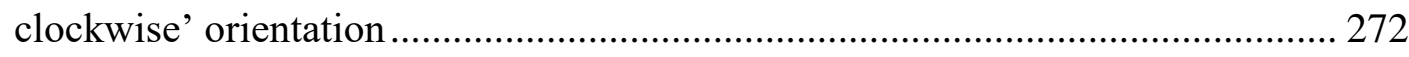

Table 50 Experimental measurements results of energy harvester I in 'anticlockwise' orientation 272 


\section{List of Abbreviations and Notations}

Abbreviations

HAVC Heating, ventilation and air conditioning

3DP Three-dimensional printing

HAWT Horizontal axis wind turbine

VAWT Vertical axis wind turbine

HAWH Horizontal axis wind harvester

OECD The Organization for Economic Co-operation and Development

FDM Finite difference method

FVM Finite volume method

FEM Finite element method

ADINA Automatic dynamic incremental non-linear analysis

DVM Discrete vortex method

SA Spalart-Allmaras

SST Shear stress transport

AR Aspect ratio

TSR Tip speed ratio 
SR

emf

CAD

CAE

$\mathrm{AM}$

FFF

PLA

ABS

STL

RPM

CFD

LED

FDM

SLA

SLS

EBM
Size ratio

Electromotive force

Computer-aided design

Computer-aided engineering

Additive manufacturing

Fused Filament Fabrication

Polylactic acid

Acrylonitrile butadiene styrene

STereoLithography

Revolution per minute

Computational fluid dynamics

Light-emitting diode

Fused Deposition Modeling

Sterolithography Apparatus

Selective Laser Sintering

Electron Beam Melting 


\section{Notations}

\begin{tabular}{|c|c|}
\hline $\mathrm{P}_{\text {wind }}$ & Wind power \\
\hline $\mathrm{E}_{\text {wind }}$ & Wind energy \\
\hline$F$ & Net force on airfoil \\
\hline$L$ & Lift force on airfoil \\
\hline$D$ & Drag force on airfoil \\
\hline$C_{L}$ & Lift coefficient \\
\hline$C_{D}$ & Drag coefficient \\
\hline$\rho$ & Air density \\
\hline$v$ & Air flow velocity \\
\hline$c$ & Chord length \\
\hline$M$ & Pitching moment \\
\hline$C_{M}$ & Pitching moment coefficient \\
\hline$\lambda$ & Tip speed ratio \\
\hline$\omega$ & Angular velocity of harvester/turbine rotor \\
\hline$R$ & Radius of harvester/turbine rotor \\
\hline $\operatorname{Re}$ & Reynolds number \\
\hline
\end{tabular}




$\begin{array}{cl}\mu & \text { Dynamic viscosity of air } \\ D_{h} & \text { Hydraulic diameter in meter } \\ F_{\text {thrust }} & \text { Thrust force } \\ A & \text { Cross-sectional/swept area of harvester/turbine rotor } \\ \dot{m} & \text { Mass flow rate } \\ p & \text { Pressure } \\ a & \text { Axial induction factor } \\ C_{P} & \text { Power coefficient } \\ \theta & \text { Rotor angle } \\ B_{r} & \text { Bagnetic field } \\ \xi_{\mathcal{N}} & \text { Electromotive force, } \mathcal{N} \text { is number of coils } \\ \eta_{g} & \text { Electromagnetic generator efficiency } \\ E_{a} & \text { Available wind power } \\ & \\ & \\ & \end{array}$




\begin{tabular}{|c|c|}
\hline$\eta_{\text {overall }}, \eta$ & Overall energy conversion efficiency \\
\hline $\mathrm{N}$ & Number of blades \\
\hline SR & Size ratio \\
\hline $\mathrm{AR}$ & Aspect ratio \\
\hline$v_{c}$ & Corrected air flow speed \\
\hline$t$ & time \\
\hline$T$ & Torque \\
\hline$J$ & Moment of inertia \\
\hline$R e_{c}$ & Cut-in Reynolds number \\
\hline$T_{s}$ & Static torque \\
\hline$C_{T s}$ & Static torque coefficient \\
\hline$C_{T d}$ & Dynamic torque coefficient \\
\hline$E_{\text {harvester }}$ & Mechanical power of energy harvester \\
\hline$x$ & Direction of oncoming air flow \\
\hline$y$ & Radial direction of energy harvester \\
\hline$z$ & Direction of energy harvester axis \\
\hline
\end{tabular}




\section{Publication List}

1. N. Han, D. Zhao, J.U. Schluter, E.S. Goh, H. Zhao, X. Jin, Performance evaluation of 3D printed miniature electromagnetic energy harvesters driven by air flow. Appl Energy 2016;178:672-80

2. N. Han, D. Zhao, J.U. Schluter, E.S. Goh, Optimum design of miniature wind energy harvesters: static, dynamic performance and energy conversion. Appl Energy. APEN-D-17-00440, Jan 16, 2017 (Under review).

3. N. Han, D. Zhao, 'Study of static and dynamic performances of miniature Savonius-type wind energy harvesters', Energy Procedia (ICAE2016), 2017. 


\section{Abstract}

An increasing concern for energy safety and environmental sustainability has shifted energy research focus to renewable energy sources. Among these energy sources, wind energy has great potential to be harnessed. Most of natural wind energy harvesting is achieved from large scale wind turbines, which are located in less populated areas. However, there are 'man-made' wind resources being neglected or wasted in tropical countries such as Singapore, such as those associated with heating, ventilation and air conditioning (HVAC) systems. Thus there is a need for developing and manufacturing energy-efficient miniature wind energy harvesters. The present work considers the design and optimization of miniature electromagnetic energy harvesters driven by air flow. For this, both experimental and numerical investigations are performed. 10 energy harvesters are designed and fabricated by using 3D printing technology as modified versions of Savonius wind turbines coupled with an electromagnetic generator. Their axes of rotation were parallel to the ground but perpendicular to the wind flow direction. The performances of these energy harvesters are evaluated in a closed-loop wind tunnel in the Aerodynamics Laboratory, Nanyang Technological University in terms of total electrical power and overall energy conversion efficiency. Preliminary parametric measurements are conducted to investigate the effects of 1) the blade number, 2) the geometric size, 3) the aspect ratio, 4) the types of central part, 5) end plates and 6) the orientation of the energy harvester. An optimum design of the miniature energy harvester is obtained in terms of the maximum 
overall energy conversion efficiency $\left(\eta_{\max }\right)$. The optimum harvester is shown to be associated with maximum efficiency of $\eta_{\max }=6.59 \%$. It is a 3 -semi-cylindricalblade energy harvester with size ratio of one, aspect ratio of one, solid central shaft, end plates at both ends and installed with an 'anti-clockwise' orientation. Comparison is then made with the commercially widely used horizontal-axis wind turbines with similar dimensions. To simulate the experiments, 3D numerical simulations are conducted by using ANSYS CFX. The numerical model is validated first by comparing with the experimental results. Finally, the model is used to evaluate the static and dynamic performances of these harvesters and to optimize the harvester's orientation and blade shape.

In summary, miniature 3D printed electromagnetic energy harvesters driven by air flow are systematically studied. It has been shown that these energy efficient harvesters have great potential to be applied in HVAC systems.

KEY WORDS: renewable energy; wind energy; 3D printing; energy harvester; wind turbine; electromagnetic; aerodynamics; 


\section{Chapter 1. Introduction}

\subsection{Background}

With increasing concern for energy safety and environmental impact caused by greenhouse gas emission, as well as rapidly depletion of fossil fuels, renewable energy such as wind power have attracted much research and industry attention during the past 30 years [2]. Such renewable energy resources have been extensively and intensively studied and explored to be utilized as one of the main contributors to replace fossil fuels.

Wind energy has become one of the dominant renewable energy resources [3], for its wide availability and economic viability. Many countries such as China, USA and Germany have invested heavily on large-scale commercial wind farms, both onshore and offshore [4]. Indeed, these large-scale wind turbines provide strong driving force to the development and expansion of wind power industry. Wind turbines for commercial applications reported [1] generally have swept area larger than $100 \mathrm{~m}^{2}$ in order to capture larger air mass and attain high efficiency. However, there are other wind energy resources receiving little attention, such as 'man-made wind' from HVAC (heating, ventilation, and air-conditioning) exhaust systems or complex air flow patterns associated with urban geometries like building rooftop. Harvesting this 'man-made wind' power is challenging due to lack of energyefficient energy harvesters to be applied in limited space available which its swept area at $1 \mathrm{~m}^{2}$ scale. Therefore, there is a recent interest on developing miniature 
energy-efficient wind harvesters to harness electricity from the 'man-made' air flow. This partially motivated the present study to explore these possibilities by designing miniature energy harvesters that can harness energy from ducted air flow in a limited space available.

It has been shown that the energy conversion efficiency of conventional horizontal axis wind turbines (HAWTs) is decreased, as its size is reduced. It is not energyefficient to apply miniature HAWTs in HVAC exhaust systems. Thus there is an interest to develop and design an alternative configuration of wind turbines, which can be explored for energy harvesting from the 'man-made wind' in HVAC systems. This also partially motivated the present study, i.e. to investigate the alternative configuration of wind turbines, besides the conventional HAWTs.

\subsection{Objectives}

The main objectives of the present study include:

1) To design and apply 3D printing technology to fabricate energyefficient miniature Savonius-like energy harvester

2) To evaluate the performances of the Savonius-like wind harvesters.

3) To identify and evaluate critical design parameters affecting the performances of these miniature harvesters.

4) To conduct parametric studies on the harvesters to gain insights on the overall energy conversion efficiency, static and dynamic performances. 
5) To identify the optimum design of the miniature wind harvester.

\subsection{Contents and Structure}

The thesis consists of five chapters, which are briefly summarized as:

1) Introduction. This chapter briefly describes the background on wind power in renewable energy sector. And main research objectives are discussed.

2) Literature review. This chapter reviews previous and on-going researches on wind turbines. Emphasis is placed on summarizing the experimental and numerical investigations conducted on Savonius wind turbines. In addition, renewable energy utilization, and the development and expansion of wind energy industry are discussed.

3) Research methodology. This chapter describes the methodology employed in this study. Before describing the experimental and numerical approaches, how to characterize wind harvester's static and dynamic performances is discussed. Finally, the design and fabrication of the energy harvesters are described.

4) Results and discussion. Experimental and numerical results on preliminary parametric studies are presented. Insightful discussion on the 
effects of critical parameters on these energy harvesters' performance is provided. Emphasis is placed on the effects of the harvester installation orientation and the blade shape, aimed to further improve the miniature harvesters' performance. Finally, comparison with conventional wind energy harvesters is made to confirm that the present design/harvester performs much better in terms of overall energy conversion efficiency.

5) Conclusions and future work. In this chapter, the key findings of the current research are summarized. Optimum design is identified and listed. In addition, further studies on how to improve the miniature harvester's performance and how to rapidly fabricate the energy-efficient miniature harvesters is proposed. 


\section{Chapter 2. Literature Review}

\subsection{Renewable energy}

Energy is an essential part of human life, and affecting many sectors including residence, transportation, agriculture, commercial and industrial activities, etc. Due to rapidly increasing demand for power generation, heat production and transport fuels manufacture, the world primary energy demand is increased by $55 \%$ between 1990 and 2013. It is projected to grow by $45 \%$ under the Current Policies Scenario, which continues the implementation of existing energy measures and policies; $32 \%$ under the New Policies Scenario, which assumes more cautious policies implementation are proposed; and $12 \%$ under the 450 Scenario which has achieved $50 \%$ probability of limiting global temperature increase within 2 degree Celsius [1]. The strong growth in energy demand has triggered increased concern for energy security and environment sustainability. Together with constantly growing demand, it has shaped a new craving for alternative energy sources that are able to supply for escalating demand with minimal impact on the environment.

Most of alternative energy sources being considered are renewable ones, including solar, wind, wave, biomass, geothermal and hydro energies [2]. Renewable energy supplied around $19.1 \%$ of energy consumption globally in 2013 , and contributed $22.8 \%$ of global electricity generation by the end of 2014 (Fig.1). Renewable energies' contributions in terms of global energy consumption and electricity generation both maintain strong growths for many decades [3]. Considering global 
total primary energy demand, the share of renewable energies holds at about $15 \%$ throughout the period to 2040 under the Current Policies Scenario; increases to $19 \%$ in 2040 under the New Policies Scenario; and has to reach nearly 30\% to achieve goals set under the 450 Scenario [1]. The importance of renewable energy is clearly demonstrated.

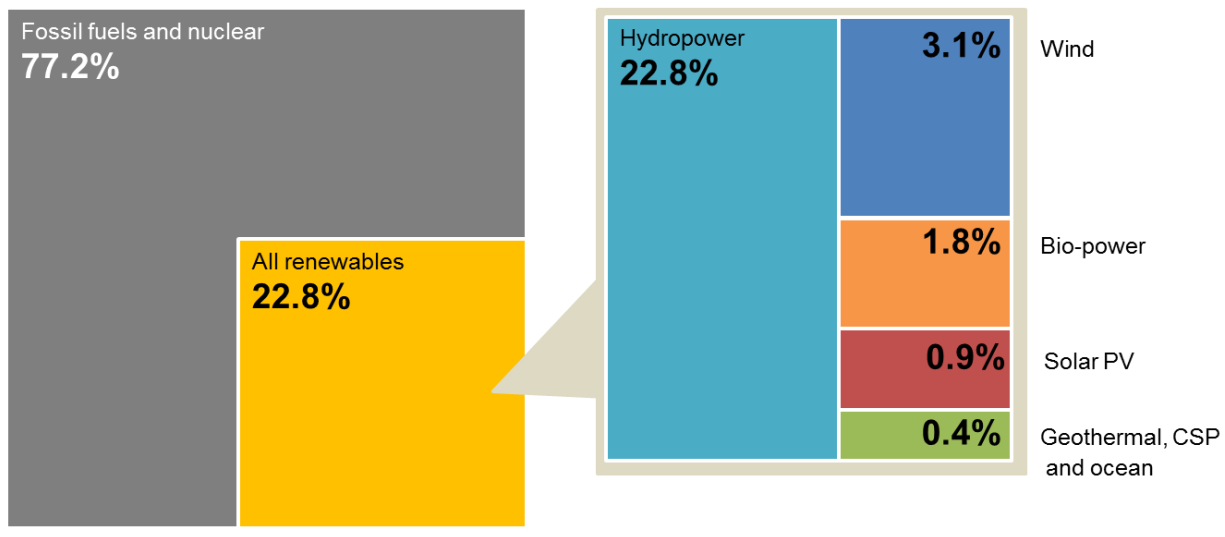

Fig.1 Estimated renewable energy share of global electricity production, end-2014. Adapted from Ref.[3].

Among various renewable energy sources, wind energy and hydro power are the most dominant energy sources in terms of energy generation. Wind energy becomes the most widely implemented non-hydro electricity-generating renewable energy source globally [4]. Examining wind energy industry by regions, it reveals that OECD (Organization for Economic Co-operation and Development) Europe is the leading player in offshore wind generation industry, while OECD Americas, OECD Europe and China are all very active in onshore wind power industry [5].

Due to the fact that these different countries are driven by the major motivations such as preserving environmental sustainability and reducing greenhouse gases 
emission, global wind power generation showed a rapid growth during the past two decades (Fig.2) [6]. Under such high growth rate, the global cumulative wind capacity is expected to reach $1684074 \mathrm{MW}$ under new policy scenario by the year 2050, and corresponding capacity under moderate scenario and advanced scenario are $2672231 \mathrm{MW}$ and $4042475 \mathrm{MW}$ respectively (Fig.3) [7].
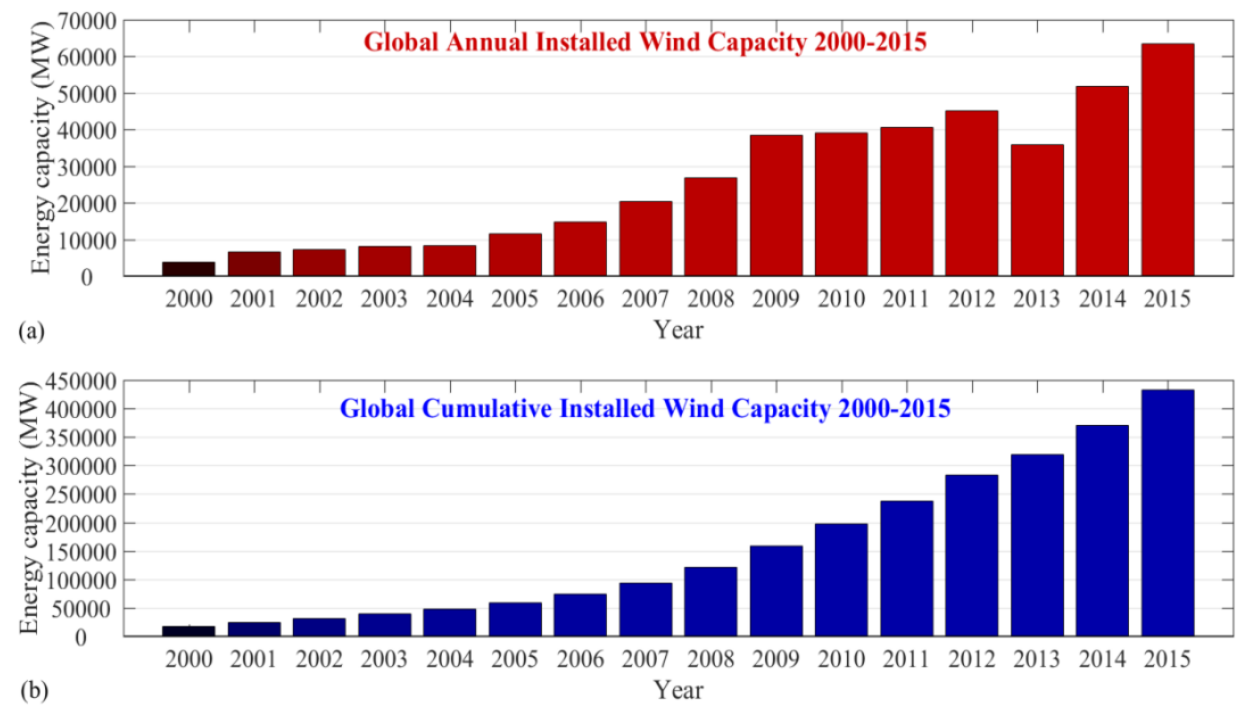

Fig.2(a) Global annual installed wind capacity 2000-2015. (b) Global cumulative installed wind capacity 2000-2015. Adapted from Ref.[6].

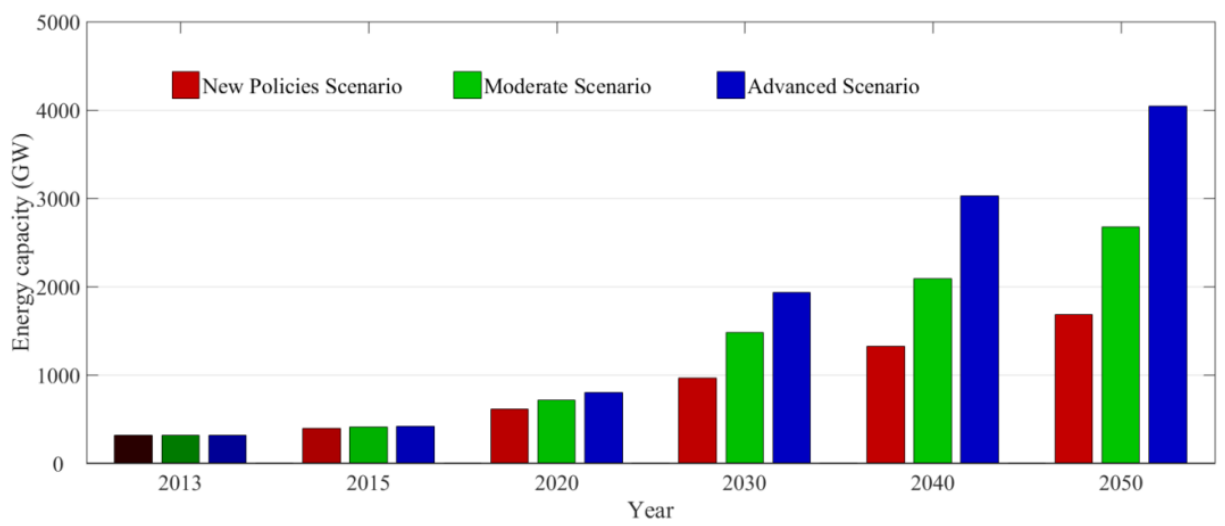

Fig.3 Global cumulative wind power capacity projection (GW). Adapted from Ref.[7]. 


\subsection{Wind energy harvesting}

Wind power has been used in the past in a few different ways, such as sailing, windmills and wind turbine, etc. In modern days, large-scale wind turbine is an energy-converting device that transforms the wind energy into mechanical energy and in turn into electrical energy [8].

Wind energy is kinetic energy of flowing fluid, the air. The amount of available wind kinetic energy depends on two factors, air mass and air flow speed. Instead of measuring air mass, it is more convenient to measure air density, thus it is more practical to find wind power rather than wind energy (Eq. ( 1)) [9]. Additionally, wind power density is another comprehensive index to evaluate the wind resource at a particular location. It is the available wind power in moving air through a unit area of perpendicular cross-sectional plane within a period[8].It can be used to evaluate the effectiveness of wind energy harvester in utilization of space.

$P_{\text {wind }}=\frac{\rho A v^{3}}{2}, \quad E_{\text {wind }}=\frac{m v^{2}}{2}$

where $P_{\text {wind }}$ and $E_{\text {wind }}$ denotes wind power and wind energy, respectively. $\rho$ is air density, $A$ is cross-sectional area, $m$ is air mass and $v$ is air speed. 


\subsubsection{Wind turbine aerodynamics}

For efficient conversion of wind energy into mechanical energy of turbine, modern wind turbine rotors are generally made into airfoil shape. Due to the airfoil curvature, air flowing along upper curvature will be at a higher velocity due to longer path traveled by air particles compared with lower streamlines. Based on Bernoulli's theorem, the higher velocity is compensated by a reduction in pressure. The pressure difference between the two surfaces of airfoil will generate a lift force $L$. Meanwhile, there will be a drag force $D$ on the airfoil exerted by air flow. The net force $F$ on the airfoil will be the resultant force of drag and lift forces $[9,10]$.

The lift is the force perpendicular to direction of oncoming airflow, and is formed due to pressure difference between the upper and lower airfoil surfaces [11]. In contrast, drag force is the force being parallel to the direction of oncoming air flow [11]. It is formed partially due to the unequal pressure on the airfoil surfaces, and partially due to the viscous friction forces on the surfaces. Lift coefficient $\left(C_{L}\right)$, drag coefficient $\left(C_{D}\right)$ and momentum coefficient $\left(C_{M}\right)$ are used to describe these forces, and are defined as:

$$
C_{L}=\frac{L}{1 / 2 \rho v^{2} c}, \quad C_{D}=\frac{D}{1 / 2 \rho c^{2} c}, \quad C_{M}=\frac{M}{1 / 2 \rho v^{2} c^{2}}
$$

where $\rho$ is the air density, $v$ is the air flow velocity and $c$ is the chord length. 


\subsubsection{Tip speed ratio}

The tip speed ratio $(\lambda)$ is another important parameter of wind turbine, and it is defined as the ratio of the blade tip tangential speed to the speed of fluid flow. Its definition can be expressed as:

$\lambda=\frac{\omega R}{v}=\frac{\omega R}{\left(\mu R e / \rho D_{h}\right)}$

where $\omega$ is the angular velocity of the wind turbine rotor, $R$ is the radius of the turbine rotor and $v$ is the wind speed. If tip speed ratio is expressed in Reynolds number, $\mathcal{D}_{H}$ is the hydraulic diameter of the wind tunnel in meter. $\mu$ is fluid dynamic viscosity.Generally a higher tip speed ratio indicates the wind turbine is associated with higher efficiency, but a higher level of noise will be produced as well.

\subsubsection{Maximum ideal efficiency}

In 1920, A. Betz predicted the ideal and frictionless efficiency of a wind turbine by applying linear momentum theory a simple one-dimensional model [11]. The model consists of a rotor represented by a uniform "actuator disk" confined in the assumed control volume. The control volume is defined by the surface and cross sections of a stream tube in which the fluid passes through the rotor disk. The rotor "actuator disk" creates pressure discontinuity of fluid flowing through it. A simple schematic illustration of the model is shown in Fig.4. 


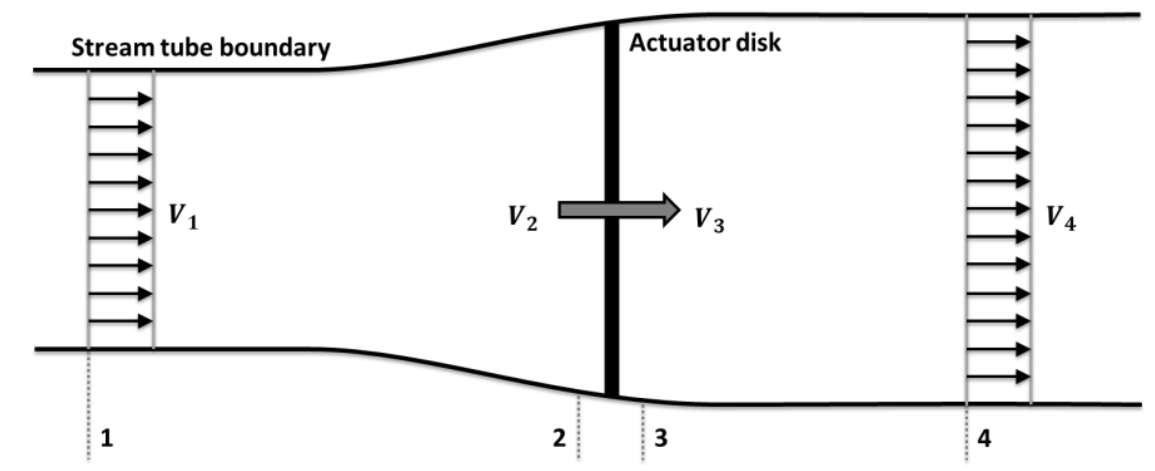

Fig.4 Control volume for actuator disk model of wind turbine. $V$ is air velocity; 1, 2,3 and 4 indicate locations.

This analysis based on actuator disk model adopts the following assumptions:

- The fluid flow is ideal across the control volume, means it is steady, homogenous, inviscid, incompressible and irrotational.

- There are an infinite number of blades, thus fits the actuator disk description.

- Both flow and trust are uniform across the disk area.

- Undisturbed ambient static pressure is assumed for the static pressure far upstream and downstream of the rotor disk.

In order to attain the net force on the contents of the control volume, the conservation of linear momentum is applied. The net force is equal in magnitude but in opposite direction to the trust, $F_{\text {thrust }}$, which is the force exerted on the turbine by the wind. For one-dimensional, incompressible and time-invariant flow, as linear momentum is conserved, the thrust can be obtained from the change in momentum of air flow: $F_{\text {thrust }}=V_{1}(\rho A V)_{1}-V_{4}(\rho A V)_{4}$, where the subscript is indicating cross section location as labeled in Fig.4, $\rho$ is the air density, $A$ is the 
cross sectional area of the rotor disk and $V$ is the air velocity of the flow. Assume the flow is steady, and pressures far upstream and far downstream are equal $\left(p_{1}=p_{4}\right)$, and the Bernoulli function is applied to upstream and downstream of the disk. Thrust can also be expressed as $F_{\text {thrust }}=A_{2}\left(P_{2}-P_{3}\right)=\frac{1}{2} \rho A_{2}\left(V_{1}^{2}-V_{4}^{2}\right)$.

An axial induction factor, $a$, is defined to express the fractional decrease in wind velocity between the free stream and the rotor disk: $a=\frac{V_{1}-V_{2}}{V_{1}}$. The maximum possible value of $a$ is $a=1 / 2$, as it requires $V_{3}$ to decrease to zero. Therefore, the thrust at the turbine plane can be written as: $F_{\text {thrust }}=\frac{1}{2} \rho A V_{1}^{2}[4 a(1-a)]$ The power coefficient, defined as the ratio of power extracted to the total power available, can be defined in terms of $a$ also: $C_{p}=4 a(1-a)^{2}$.

Examining the power coefficient at different values of axial induction factor, it can be seen that power coefficient reaches a maximum value of 0.593 , which is known as the Betz limit for an ideal frictionless turbine. This theoretical maximum efficiency is difficult to be achieved practically for many reasons, such as:

- Pressure distribution at the rotor disc is not uniform.

- Viscous effect will produce aerodynamic drag.

- Vortices formed at the blades tips will lead to energy loss.

Betz's analysis on wind turbine efficiency is not limited to any particular type of wind turbine [12]. Fig.5 is showing the relationships between the turbine power coefficient and the tip speed ratios for different types of wind turbines. It is obvious 
that for different wind turbine designs, the maximum power coefficient is achieved at different tip speed ratios [13]. This means the suitability of wind turbine design to a particular working environment must be considered. Regarding to the present study, the tentative use of energy harvester is associated with lower wind speed and small installation space, thus it is unlikely to achieve high tip speed ratio. Hence, Savonius rotor is taken as the primary candidate for the research.

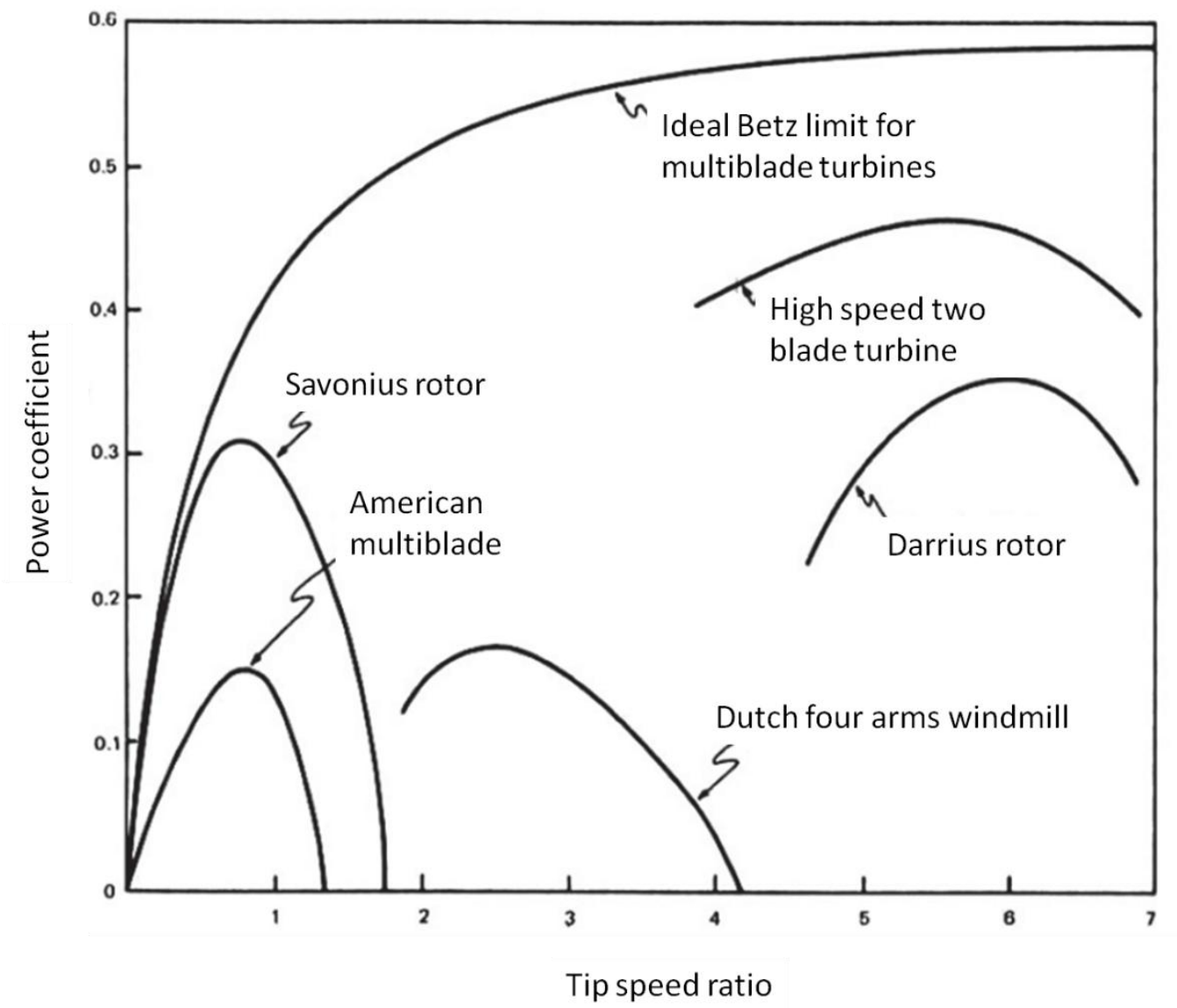

Fig.5 The power coefficient as a function of tip speed ratio for different wind turbine machines designs. Adapted from Ref. [13]. Note that in some previous publications (Ref. [12]), the efficiency curves of the American multiblade and the Savonius designs were inadvertently switched. 


\subsubsection{Wind turbine classification}

Wind turbine classification can be based on turbine axis of rotation, relative position of wind turbine, wind turbine capacity, type of generator used, the power supply mode, and the turbine location [8]. The classification method being used in this study is based on turbine axis of rotation.

Horizontal axis wind turbines (HAWTs) are those whose axes of rotation are horizontal or nearly horizontal to the ground and almost parallel to the wind direction[9]. Most of the commercial wind turbines are HAWTs [10, 11]. Those include the most commonly seen propeller turbines, which are lift-type that work based on the lift force on the blades [9]. Unlike vertical axis wind turbines (VAWTs),HAWTs are preferred in electricity generation due to relatively higher energy conversion efficiencies [14].

There are a number of advantages involved in HAWTs [10]:

- HAWTs are the most stable and commercially applied designs.

- HAWTs produces power resulted from relatively lower cut-in wind speed and involve with higher energy efficiency.

- It is possible to utilize them at higher elevation using taller towers.

- The system performs better under fluctuating wind speed due to better angle of attack control.

- There is easier furling by turning the rotor away from wind stream.

Unfortunately, there are some inherent drawbacks as well [10]: 
- HAWTs require yaw drives to turn the turbine toward oncoming wind.

- Stronger structural support is needed for heavy generator and gearbox.

- Installation and maintenance costs are higher due to greater tower height.

- Taller towers are detrimental to wind farms' visual acceptability.

In contrast, when a wind turbine's axis of rotation is vertical to the ground and nearly perpendicular to the wind direction, the turbine is an example of VAWT [9, $10]$.

Distinct advantages of VAWTs over HAWTs are[10]:

- VAWTs are insensitive to wind direction, thus no yaw control is necessary.

- Structural requirement is less stringent as heavy components like gearbox and generators can be placed close to ground level.

- Maintenance is easier at ground level.

- Pitch control is not required for VAWTs.

However, VAWTs are not well accepted commercially due to some major disadvantages [10] as following:

- Generally, VAWTs are not self-starting. Hence additional motor may be required.

- The turbines are closer to the ground, where wind speed is lower than higher elevations. 
- Efficiency is limited as the blades have to pass through aerodynamically dead zones in a complete cycle.

- Guy wires may be required to support the structure, and may cause inconveniences for installation and maintenance.

Most of wind energy industry share is contributed by large-scale turbine harvesters, which are generally HAWTs. VAWTs also attract increasing attention for their utilization as small to medium scale wind energy harvesting units[15].

\subsubsection{Urban wind energy}

There are urban areas where wind energy resource is abundant, and many attempts have been made to utilize urban wind energy potential. Urban wind energy utilization can be classified into a few categories as shown in Table 1. However, due to the fact that urban areas are much more populated, many factors need to be taken into cautious consideration, including environmental and social impact of wind turbine installation.

Table 1 Urban wind energy utilization categories. Adapted from Ref. [16].

Category Remarks

Small wind energy "Retrofitting" and building-mounted wind turbines

Large wind energy $\quad$ Stand-alone wind turbines

Building-integrated Sustainable building designed with wind energy in wind turbines mind 
Assessment of on-site wind resource is essential in determining feasibility of wind turbine projects. As discussed, available wind energy for harvesting is very sensitive to the wind speed. A brief summary of wind speeds in relation to commercial wind turbine function is given in Fig.6.

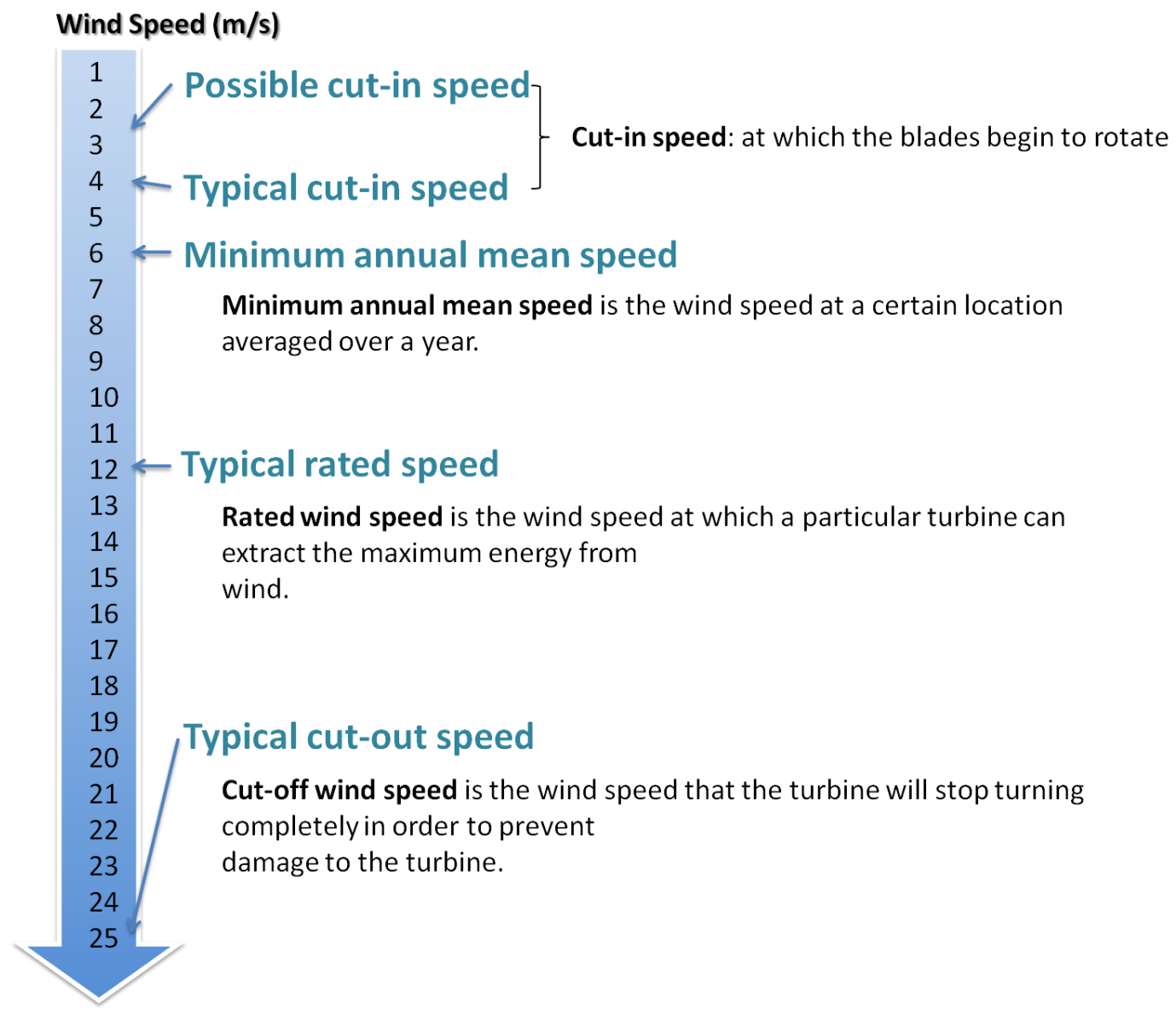

Fig.6 Specific wind speeds in relation to wind turbine operation. Adapted from Ref. [16].

However, not all urban locations are able to fulfill wind speed requirement for wind energy utilization. For the case of Singapore, harnessing wind energy is not a 
viable option as Singapore's average wind speed is about $2 \mathrm{~m} / \mathrm{s}$ to $3 \mathrm{~m} / \mathrm{s}$ [17], and most commercial wind farms require mean annual wind speed above $6.0 \mathrm{~m} / \mathrm{s}$ [16]. Therefore, instead of harnessing natural wind energy in urban areas, which tends to have low and unstable wind flow speed, harnessing from 'man-made' wind energy resources will be a more viable choice.

\subsection{Studies on Savonius wind turbines}

A Savonius rotor was named after its inventor, Siguard J. Savonius in 1920s. Its simplest form consists of two half-cylinder blades fixed to a shaft in the form that its cross section looked like a letter 'S' [9]. There are many variations in Savonius type wind turbine configurations, such as introducing an empty central gap where the blades are joined, implementing end plates, changing blades number or rotor stage number, and modifying the shape of the end plates, etc [18]. The Savonius rotor was widely considered as drag-driven device [9]. However, it was observed that at low angles of attack that the lift force also contributed to rotor rotation [19], thus Savonius rotor could exceed the power coefficient limit established for purely drag-driven machines [11]. Many investigations were done in improving the Savonius rotor's performance efficiency. It is to be noted that as the air-driven energy harvester under investigation has some similarities with Savonius-type wind turbine, therefore literature review on Savonius wind turbines was conducted and presented in details. 
It must be also noted that most of the studies on Savonius wind turbines were conducted in the condition that the turbines were installed as vertical axis wind turbine. However, few studies had been reported on investigation of alternative configuration of Savonius-type wind turbine, which has its axis of rotation perpendicular to oncoming air flow direction but parallel with the ground. Additionally, the study results found in literature were still at meter scale, meaning they were too large to be installed in HVAC ventilation systems. In the HVAC ventilation exhaust system, the duct diameters are generally smaller than 60 inches (duct areas are smaller than $7 \mathrm{~m}^{2}$ ) [20]. To the best knowledge of author, few studies were reported on energy harvester with smaller scales. Hence, there is a need to further reduce device dimension in order to accommodate the installation requirements of energy harvesters. The review of Savonius-type wind turbine would be a starting point to investigate miniature scale energy harvester. This partially motivates the present study to explore this alternative configuration of Savonius-type wind turbine.

Numerous studies on Savonius-type VAWTs had been carried out since its invention in 1920s [21]. Its operation mechanism was very well explained by various groups, including Fernando and Modi [22], Saha et al. [23] and Nakajima et al. [24, 25]. In recent years, theoretical, experimental and simulation achievements all significantly expanded the concept and applications of Savonius wind turbine, by optimizing turbine and accessory parts design to improve power conversion efficiency. 


\subsubsection{Experimental studies}

Experimental investigations on Savonius wind turbine performance are abundant, started with field tests, closed- and open-section wind tunnel tests and hydrodynamic canal measurements. Simonds and Bodek performed measurements on Savonius wind turbine in field [26]. Mojola designed Savonius windmill [27] and Rabah and Osawa built a Savonius wind pump [28], and both designs were tested in field. Sheldahl et al. [29] and Alexander et al. [30] both performed closedsection wind tunnel tests on Savonius wind turbines in 1970s. Open-section wind tunnel tests were also conducted: Fujisawa [23] to analyze torque development mechanism in Savonius rotor; Hayashi et al. [32] tested multi-stage Savonius rotor; Saha et al. [33,34] explored different blade shapes and design configurations. Nakajima et al. [24, 25] also conducted hydrodynamic canal tests to evaluate performance of environmentally friendly Savonius rotor. By these various methods, meaningful results were gathered.

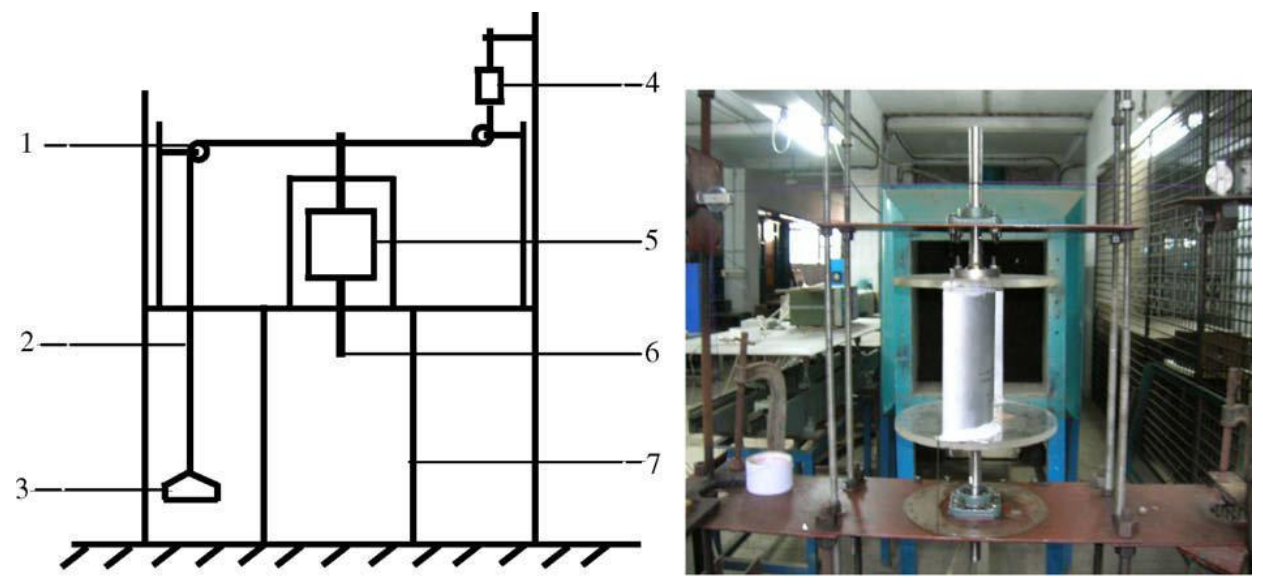

1. Pulley 2. Nylon string 3. Weighing pan 4. Spring balance 5. Savonius rotor 6. Shaft 7. Structure 
Fig.7 Schematic diagram of the experimental set-up for Savonius rotor testing built by M. A. Kamoji et al. (Ref. [35]).

More recently, Kamoji et al. had built an experimental test platform and conducted parametric study on effect of overlap ratio, blade arc angle, aspect ratio and Reynolds number, and reported highest power coefficient of 0.21 [35] (Fig.7). Roy and Saha studied a two-bladed Savonius turbine and achieved maximum power coefficient of 0.348 in open type wind tunnel test section [37]. Driss et al. reported their findings on effects of bucket design and incidence angle on performance of an incurved Savonius wind turbine which is tested in open wind tunnel $[38,39]$. Kamoji et al. also studied the performance of helical Savonius wind turbine, especially the effects of end plates and overlap ratio were studied and attained the maximum power coefficient of 0.174 [40]. Damak et al. also reported more efficient performance of helical Savonius rotor as compared to conversional Savonius turbines [41]. Jeon et al. reported $36 \%$ increment in power coefficient by studying effect of end plates size and shapes on helical Savonius turbine [42] (as shown in Fig.8). Golecha et al. [43] and Mohamed et al. [44]both reported on the enhancing effect of obstacle shielding and the highest increment in power output is $50 \%$. Altan et al. presented a $20 \%$ increment of power coefficient by introducing an additional straight blade [45]. Additionally, Roy and Saha had studied the blockage effect in wind tunnel tests to improve accuracy of Savonius wind turbine study [46]. Savonius turbine is also installed with other types of wind turbines to achieve better energy conversion efficiencies, for instance, Bhuyan and Biswas reported a hybrid H-Savonius wind rotor that achieves maximum power coefficient of 0.28 
[47] and Gupta et al. also reported a Savonius-Darrieus rotor with an improved efficiency of 0.51 [48].
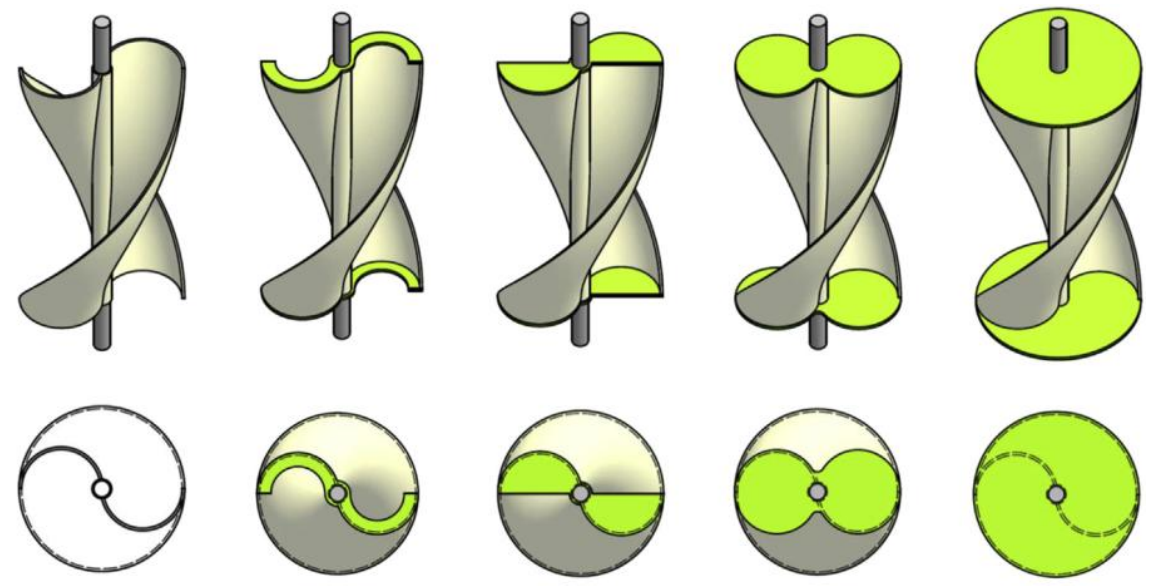

(a) No end plate

(b) End plate \#1

(c) End plate \#2

(d) End plate \#3

(e) End plate \#4

Fig.8 Views of helical Savonius rotors with various shapes and sizes of end plates by Jeon et al. (Ref. [42]).

\subsubsection{Numerical studies}

Computational work on Savonius wind turbine was also intensively done, and was initiated by Wilson et al. [49] and Van Dusen and Kirchhoff [50] in 1970s. Jaohindy et al. has studied the effect of aspect ratio and analyzed the transient forces acting on the rotor [51]. Zhou et al. and Kacprazk et al. both studied the flow field around Savonius wind turbines and suggested changes in blade shape to achieve better performance than conventional semi-circular shape blade [52]. McTavish et al. studied the static and dynamic performance of Savonius wind turbine that consists of several asymmetric vertically-stacked stages, and characteristic static and dynamic torque behavior of rotor are obtained [53]. Grouping of several Savonius turbines to achieve higher efficiency was also 
numerically studied. Shigetomi et al. studied the interactive flow field around two Savonius turbines [54], and El-Baz et al. reported $44 \%$ increment of power coefficient when three Savonius turbines were arranged in a triangular pattern [55]. Tartuferi investigated on new blade shapes that achieved a maximum power coefficient of 0.301 and curtain systems that improved power output by $20 \%$ [56]. Altan and Atilgan also investigated on enhancement effect of curtain system by studying the fluid flow numerically and experimentally [57]. Different methods were adapted to study the performance of Savonius wind turbine. Kawamura and Sato [58] used finite difference method (FDM) to solve the flow around the wind turbine. Finite volume method (FVM) was more popular, especially with widely accepted software packages like ANSYS-Fluent and ANSYS-CFX, to analyze the performance of Savonius rotor [59, 60]. Finite element method (FEM) based software packages like COSMOS-floworks and ADINA (Automatic dynamic incremental non-linear analysis) were reported for solving the flow problems around Savonius rotors $[61,62]$. Other special methods were also reported to analyze flow over Savonius rotor, such as discrete vortex method (DVM). Ogawa [63], Kotb et al. [65] both used DVM to study flow field about Savonius rotors. Modi et al. [64] studied aerodynamics and wake of turbine by using DVM. Fujisawa [66] performed both DVM calculation and velocity measurements. Afungchui et al. had achieved good agreement with experimental data using DVM [67]. Alessandro et al. proposed a mathematical model to study the complex flow field around the Savonius rotor, and gained meaningful insights on flow pattern, turbulent intensity, velocity magnitude profile and central vortex [68]. 
Turbulence models for rotor simulation were also studied. The Spalart-Allmaras (SA) model, being a simple one-equation turbulence model, was less sensitive in the near walls treatment around the turbine rotor as its near wall gradients of the transported variable were much smaller. The standard k- $\varepsilon$ model [69] had shown ability to produce better results than SA model for turbine analysis [70]. However, the computational results might not guarantee the accuracy of k- $\varepsilon$ model under assumptions like unsteadiness and two-dimensional constraint [71-74]. New models like renormalization of the $\mathrm{k}-\varepsilon$ model (RNG $\mathrm{k}-\varepsilon$ model) and the realizable $\mathrm{k}-\varepsilon$ model which gave a new formulation for turbulent viscosity and dissipation rate had found to be significant improvements over the standard k- $\varepsilon$ and SA models [75]. The shear stress transport (SST) k- $\omega$ model is a two-equation eddy viscosity model that combines advantages of both $\mathrm{k}-\varepsilon$ formulation for free stream flow and k- $\omega$ formulations in the rotor boundary layer [76, 77]. Further, Plourde et al. [78] had achieved very good agreement with the experimental results by using threedimensional SST k- $\omega$ model. Comparative study done by Dobrev et al. [79] showed that 2D SST k- $\omega$ model is over-predicting, whereas 3D SST k- $\omega$ model shows better accuracy but slightly under-predicts the experimental results. Nasef et al. also concluded that Shear Stress Transport (SST) gives more accurate results by studying the static and dynamic performances of Savonius rotor [80].

\subsubsection{Investigations on influencing parameters}

For last 30-40 years, computational studies along with the experiments had been carried out to improve the efficiency of the Savonius rotor. Many influencing 
design parameters were studied, and the turbine performance was enhanced significantly. Some of these influencing design parameters being studied were [14, 18]:

- $\quad$ Tip speed ratio (TSR, $\lambda$ ): is defined as ratio of tip speed to the free wind speed.

- Aspect ratio $(A R)$ : is defined as the ratio of height of the rotor to its diameter.

- Overlap ratio: is defined as the ratio of the overlap distance between two blades to the chord length of the blade.

- Rotor angle $(\theta)$ : is defined as the angle between the chord of the blade to the direction of air flow.

- Reynolds number $(R e)$ : is the non-dimensional parameter for defining the flow characteristics around the wind turbine.

- Blockage factor $(f)$ : is an important factor to characterize test conditions in wind tunnel setting.

- Number of blades: is an effective parameter depending upon the operating conditions. Normally two, three and four-bladed rotors have been used.

- Blade shape: is referring to the cross-sectional profile shape.

- End plates: prevent the escape of air from the concave side of the blade to the external flow. Thus the pressure difference between the concave and convex sides of the blades can be maintained at satisfactory levels.

- Interference of shaft and other accessories 
It is to be noted that other augmentation techniques were utilized to further improve Savonius wind turbine performance. These techniques include [14]:

- Use of multi-staging

- Use of curtain design

- Use of wind shields

- Use of convergent nozzle 


\section{Chapter 3. Research Methodology}

\subsection{Energy harvester performance evaluation and hypothesis}

To utilize wind energy in urban areas, miniature wind turbines installed in dwelling areas are to be designed to avoid complicated factors and distortion of flow fields. The potential installation sites could be high-building roof and HVAC ventilation exhaust system. To characterize and evaluate the performance of designed airdriven energy harvesters, static and dynamic performances are to be considered.

Before establishing a framework to evaluate the performance of energy harvester, the following hypotheses are made:

- The air flow is uniform and the air flow rate measured is taken as the rate at which the air flows through the whole swept area of the energy harvester.

- The rotation of energy harvester is uniform throughout whole revolution, thus the time evolution of electromotive force induced is perfectly sinusoidal.

- The effects of temperature and humidity are treated as consistent for all the tests, thus does not affect the performance of energy harvester models.

As a wind energy harvester interacts with moving air flow, kinetic energy of wind is extracted by the harvester and converted into mechanical energy of the turbine rotor, and eventually into electrical energy form by using induction generators. The potential wind power $E_{a}$ to be harvested can be shown as 


$$
E_{a}=\frac{A\left(\mu R e / \mathcal{D}_{H}\right)^{3}}{2 \rho^{2}}
$$

$\rho$ is air density, $A$ is energy harvester swept area, and Re is the Reynolds number: $R e=\frac{\rho v \mathcal{D}_{H}}{\mu}, \mathcal{D}_{H}$ is the hydraulic diameter of the wind tunnel in meter. $\mu$ is fluid dynamic viscosity and $\mu=1.983 \times 10^{-5} \mathrm{~N} \mathrm{~s} / \mathrm{m}^{2}$ for air.

When the wind energy is converted to electrical energy eventually, harvester power output can be assessed in terms of electrical power produced. Electrical power output $E_{e}$ can be related to available wind power by

$\boldsymbol{E}_{\boldsymbol{e}}=\boldsymbol{E}_{\boldsymbol{a}}\left(\boldsymbol{C}_{\boldsymbol{P}} \cdot \boldsymbol{\eta}_{\mathrm{g}}\right)$

where $\eta_{g}$ is generator efficiency and $C_{P}$ is the coefficient of power which is given by the ratio of air-driven energy harvester power output to wind power available[9].

The output electrical power $E_{e}$ is related to induced electromotive force $\left(\mathrm{emf}, \xi_{\mathcal{N}}\right)$ as

$E_{e}=\frac{\xi_{\mathcal{N}}^{2}}{2 R_{r}}=\frac{\xi_{r m s}^{2}}{R_{r}}$

Here $R_{r}$ is the resistance of the copper induction coil.

The induced emf $\left(\xi_{\mathcal{N}}\right)$ due to electromagnetic effect is based on Faraday's law [81] in integral form as:

$\xi_{\mathcal{N}}=\mathcal{N} \oint_{\mathcal{T}} E \cdot d s=\mathcal{N} \iint_{S}(\nabla \times E) \cdot n d A=-\mathcal{N} \frac{\partial}{\partial t} \iint_{S} B \cdot n d A$

where $\mathcal{T}$ is a closed curve, $S$ is the surface bounded by it, $E$ is the electric field and $B$ is the magnetic field. It is assumed that emf generated from each turn of the coil is equal. It can be seen that $\xi_{\mathcal{N}}$ induced in the closed path equal to the negative of 
the time derivative of magnetic flux enclosed by the path. Thus the output power $E_{e}$ can be shown as:

$E_{e}=\mathcal{N}^{2} \frac{\left[\frac{\partial}{\partial t}\left(\iint_{S} B \cdot n d A\right)\right]_{\max }^{2}}{2 R_{r}}$

and it can be seen that the generated power $E_{e}$ is $\mathcal{N}^{2}$ times larger than the wound coil of copper with 1 turn, as $R_{r}$ remains constant.

The emf can be measured as voltage induced in the circuit, and its maximum value is the peak value of the voltage. Peak voltage value and induction coil resistance both can be directly measured by open-circuit or closed-circuit methods, and subsequently used in electrical power determination.

To characterize the efficiency of air-driven energy harvester, the overall efficiency can be calculated by

$\eta_{\text {overall }}=C_{P} \cdot \eta_{g}=\frac{E_{e}}{E_{a}}$

where $E_{e}$ can be obtained through voltage and resistance measurements, and $E_{a}$ can be evaluated knowing the air flow rate. 


\subsection{Experimental work}

\subsubsection{Air-driven energy harvester design}

There are many different design parameters affecting the performance of an airdriven energy harvester. In this work, the following factors are investigated for their effects on air-driven energy harvester's performance:

- Number of blades of air-driven energy harvester $(N)$

- Air-driven energy harvester geometric size (size ratio, $S R$ ), where size ratio is defined as the ratio of the size of the energy harvester under study to the size of the benchmark energy harvester.

- Air-driven energy harvester aspect ratio $(A R)$, which is the ratio of energy harvester length to the diameter of energy harvester end plate diameter.

- Type of central part, means whether the energy harvester possess a solid central shaft or an empty central gap.

- Presence or absence of end plates at both ends of harvester.

- Air-driven energy harvester orientation, which means whether the energy harvester rotates in clockwise or anti-clockwise direction.

- Blade shape: the shape of energy harvester's cross-sectional profile.

The effects of these factors are evaluated in terms of both dynamic and static performance of the energy harvesters. The dynamic performance mostly concerns with the energy conversion efficiency of the harvesters, while the static 
performance addresses more about the self-starting capabilities of the energy harvesters.

Air-driven energy harvester models are designed with software named SolidWorks, which is a 3D solid modeling computer-aided design (CAD) and computer-aided engineering (CAE) software program [82]. It is able to facilitate both design and fabrication processes of wind energy harvester models.

The proposed designs of air-driven energy harvesters are modified versions of Savonius-type wind turbines. It must be emphasized that the designed energy harvester is different from both HAWTs which have axes of rotation parallel to the ground and oncoming air flow direction, and VAWTs which have axes of rotation perpendicular to the ground and oncoming air flow direction. The energy harvesters in this study have their axes of rotation parallel to the ground but perpendicular to the oncoming air flow direction. 10 models are designed to investigate the effects of different design factors on the energy harvester performance in the preliminary parametric study, and the models are named from harvester A to J. The design factors covered in preliminary parametric study are 1) number of harvester blade $(\mathrm{N}), 2$ ) the geometric size of the harvester model (SR), 3) the aspect ratio of harvester model (AR), 4) the type of harvester central part, 5) the presence of end plates and 6) the installation orientation of energy harvester models. The effect of energy harvester orientation and blade shape are studied numerically and will be presented in later part of this thesis. An illustration of the energy harvesting system can be seen in Fig.9. 


\section{$\underline{\text { Harvester A }}$}

This model works as the benchmark test model. Other models are designed to modify one particular design parameter compared to this model. The choice of design factors, the graphical illustration of Model A design and its characteristic parameters can be found in Appendix A. Model A has three blades $(\mathrm{N}=3)$, harvester diameter the same as harvester length $(\mathrm{AR}=1)$, solid central shaft and end plates at both ends. The model is installed in a way that the concave side of the blade facing the oncoming air flow is at the lower half of the turbine, thus the energy harvester is rotating clockwisely in positive $\mathrm{z}$ direction. Unless otherwise stated, the other energy harvesters are installed in the same orientation as harvester A.

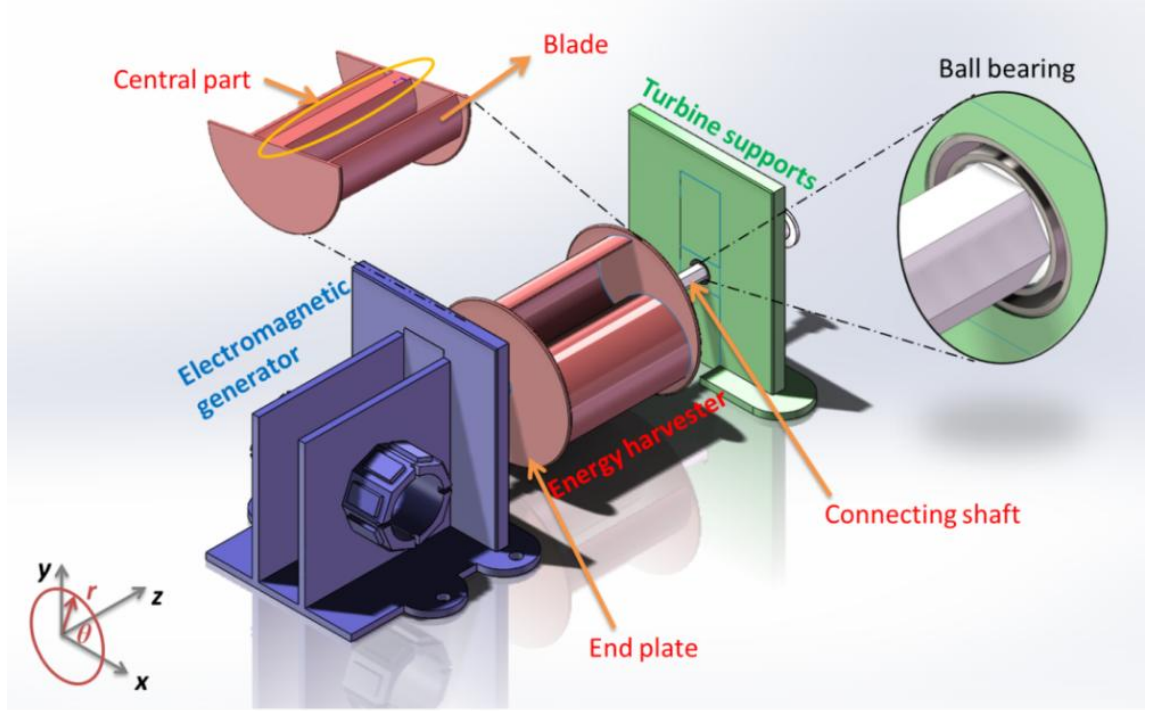

Fig.9 Illustration of air-driven energy harvesting system. 


\section{$\underline{\text { Harvester } B}$}

This model is designed to investigate the effect of blade number on the performance of wind energy harvester, and it has one less blade than harvester $\mathrm{A}$ $(\mathrm{N}=2)$. Its design factors choice, graphical illustration and characteristic parameters can be found in Appendix A.

\section{$\underline{\text { Harvester } C}$}

This model is also designed to investigate the effect of blade number on the performance of energy harvester, and it has one more blade than harvester $\mathrm{A}(\mathrm{N}=4)$. Its design factors choice, graphical illustration and characteristic parameters can be found in Appendix A.

\section{$\underline{\text { Harvester } D}$}

This model is designed to investigate the effect of the air-driven energy harvester geometric size on the performance of the energy harvester. The model is two-thirds in geometric size as compared to harvester $\mathrm{A}$, hence its size ratio is defined to be $\mathrm{SR}=2 / 3$. Its harvester length and diameter are both reduced to $2 / 3$ of those of harvester A. Its design factors choice, graphical illustration and characteristic parameters can be found in Appendix A.

\section{$\underline{\text { Harvester } E}$}

This model is designed to investigate the effect of energy harvester geometric size on its performance. This model is larger than harvester A, and both harvester length and diameter are increased simultaneously. It is four-thirds in geometric size 
as compared to that of harvester A, hence its size ratio is defined to be $S R=4 / 3$. Its design factors choice, graphical illustration and characteristic parameters can be found in Appendix A.

\section{$\underline{\text { Harvester } F}$}

This model is designed to investigate the effect of energy harvester's aspect ratio on the performance of it. Harvester $\mathrm{F}$ has the same harvester diameter as that of harvester A, but the harvester length is reduced to half of harvester A's value. Thus, its aspect ratio is $A R=1 / 2$. It is to be noted that as the harvester length is halved, its size is also reduced as compared to harvester A. Its design factors choice, graphical illustration and characteristic parameters can be found in Appendix A.

\section{$\underline{\text { Harvester } G}$}

This model is designed to investigate the effect of energy harvester's aspect ratio on the performance of it. Harvester $G$ has the same harvester length as that of harvester A, but the harvester diameter is reduced to half of harvester A's value. Thus, its aspect ratio is $A R=2$. It is to be noted that as the harvester diameter is halved, its size is also reduced as compared to harvester A. Its design factors choice, graphical illustration and characteristic parameters can be found in Appendix A.

\section{$\underline{\text { Harvester } H}$}

This model is designed to investigate the effect of type of central part where the blades are joined. Its design factors choice, graphical illustration and characteristic parameters can be found in Appendix A. It is to be noted that all the parts of this 
model are the same as those of harvester A, except that the central part is left empty in harvester $\mathrm{H}$.

\section{$\underline{\text { Harvester I }}$}

This model is designed to investigate the effect of end plates on the performance of air-driven energy harvester. The model has every part the same as harvester A, except there are no end plates on either end of the turbine harvester. Its design factors choice, graphical illustration and characteristic parameters can be found in Appendix A.

\section{$\underline{\text { Harvester J }}$}

This model is designed to study the effect of energy harvester's orientation on its performance. The design of this energy harvester model is exactly the same as harvester $\mathrm{A}$, and its design factor choice, graphical illustration and characteristic parameters can be found in Appendix A. The difference is that this model is installed in a way that the concave side of the blade facing the oncoming air flow is at the upper half of the turbine. This means the harvester model is rotating anticlockwisely in positive $\mathrm{z}$ direction (see Fig.9). This difference in orientation is illustrated in Fig.10. 

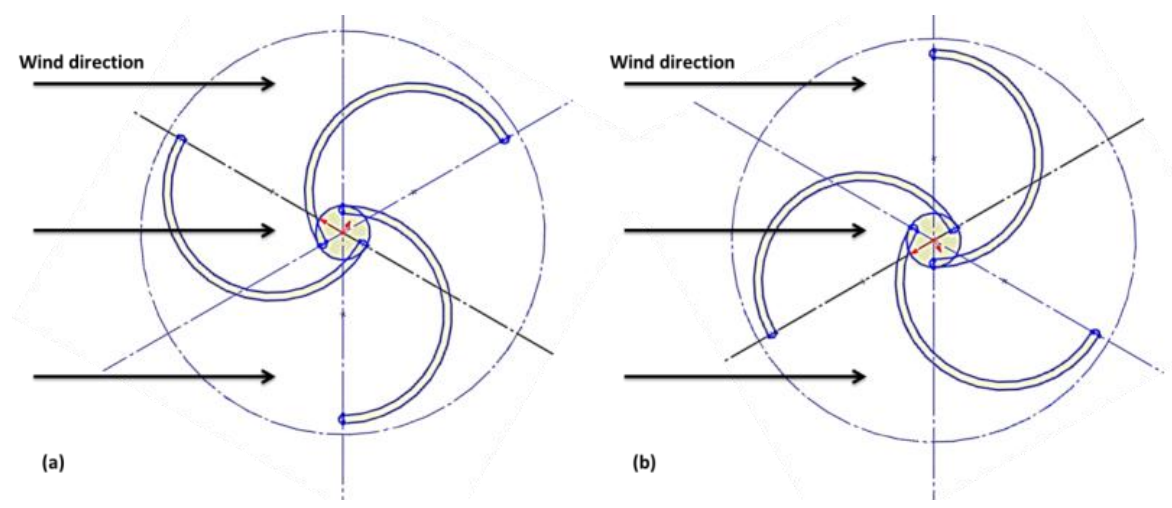

Fig.10 Orientation of (a) Model A (Lower/clockwise orientation) and (b) Model J (Upper/anti-clockwise orientation).

\section{$\underline{\text { Summary }}$}

The design parameters of these ten models for preliminary parametric study are summarized in the Table 2. 
Table 2 Specifications of energy harvester models design in preliminary parametric study.

\begin{tabular}{|c|c|c|c|c|c|c|c|c|c|c|}
\hline Harvester & A & B & C & D & $\mathbf{E}$ & $\mathbf{F}$ & G & $\mathbf{H}$ & I & J \\
\hline $\begin{array}{c}\text { No. of } \\
\text { blades (N) }\end{array}$ & 3 & 2 & 4 & 3 & 3 & 3 & 3 & 3 & 3 & 3 \\
\hline $\begin{array}{l}\text { Geometric } \\
\text { size (SR) }\end{array}$ & 1 & 1 & 1 & $2 / 3$ & $4 / 3$ & NA & NA & 1 & 1 & 1 \\
\hline $\begin{array}{c}\text { Aspect ratio } \\
(A R)\end{array}$ & 1 & 1 & 1 & 1 & 1 & $1 / 2$ & 2 & 1 & 1 & 1 \\
\hline Central gap & Solid & Solid & Solid & Solid & Solid & Solid & Solid & Empty & Solid & Solid \\
\hline End plates & Yes & Yes & Yes & Yes & Yes & Yes & Yes & Yes & No & Yes \\
\hline Orientation & clockwise & clockwise & clockwise & clockwise & clockwise & clockwise & clockwise & clockwise & clockwise & $\begin{array}{c}\text { anti- } \\
\text { clockwise }\end{array}$ \\
\hline
\end{tabular}


With understanding gained from preliminary parametric study, further investigations on effect of energy harvester installation orientation and harvester blade shape are conducted numerically. Details on these sub-case studies will be presented later.

\subsubsection{Air-driven energy harvester auxiliary parts design}

Besides energy harvester itself, other auxiliary parts are also designed to facilitate air-driven energy harvester performance testing.

\section{Air-driven energy harvester supports}

Air-driven energy harvester supports are designed to lift and support the energy harvester models, as can be seen in Fig.11a. To facilitate smooth rotation of energy harvester, ball bearings are incorporated into turbine support to greatly reduce the rotational friction between the energy harvester shaft and its support.

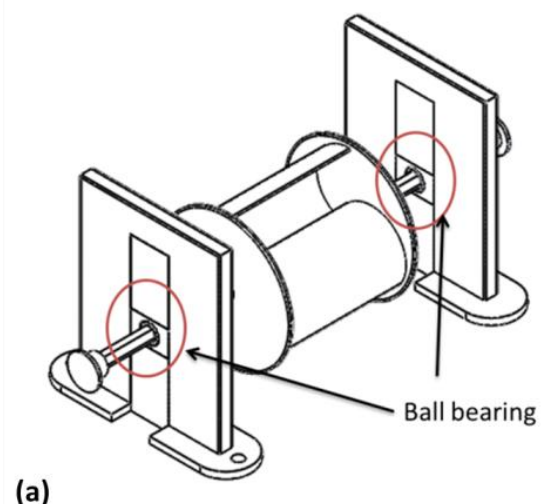

(b)

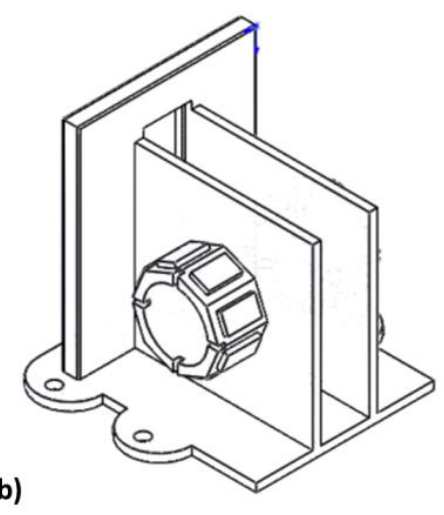

Fig.11 (a) Air-driven energy harvester support and (b) induction coil holder 


\section{$\underline{\text { Electromagnetic convertor }}$}

In order to convert energy harvested from moving air into electrical power, induction coils are used to convert mechanical energy of energy harvester into electricity via electromagnetic induction. The permanent magnet is attached onto the shaft at one end of the energy harvester, and placed in a wound of copper induction coil. When the torque exerted by the moving air onto the energy harvester exceeds the frictional torque, the harvester starts to rotate and so does the shaft and the magnet attached. The rotation of magnet causes the magnetic flux change in the induction coil, thus induced emf via mechanism discussed in Section 3.1.2. According to the Faraday's law, the emf induced in the closed path (the coil) is equal to the negative of the time rate of change of magnetic flux enclosed by the path [81]. Based on Eq. ( 7) and Eq. ( 8), we can see that for a coil having constant number of turns, the faster the energy harvester rotates, the larger the rate of magnetic flux change, and subsequently the greater the emf induced.

To build the electromagnetic convertor, an induction coil holder is designed as shown in Fig.11b, the copper coil is wounded on both sides of the holder, and the magnet rotates in the middle of it. The air-driven energy harvester, together with the turbine supports and electromagnetic generator, the whole air-driven energy harvesting system is shown inFig.9.

\section{$\underline{\text { Step height platform }}$}

To mimic the environment that the designed energy harvesters are to be installed, a step height platform is designed so that the energy harvesting system is placed on 
top of it (Fig.12). The characteristic parameters of the platform can be found in Table 3. The platform is constructed and mounted onto the base of wind tunnel test section with aluminum profiles from Prestech Industrial Automation Pte Ltd [83]. The platform attached to the wind tunnel test section, together with the energy harvesting system installed on it, can be seen in Fig.13.

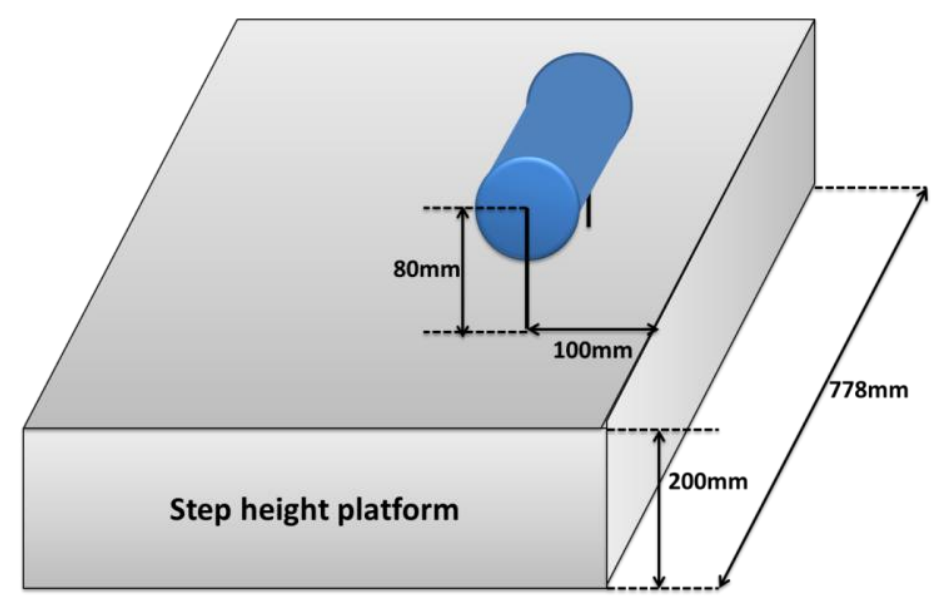

Fig.12 Schematic diagram illustrating air-driven energy harvesting system installation on the step height platform.

Table 3 Characteristic parameters of step height platform.

\begin{tabular}{ll}
\hline Parameter & Value \\
\hline Bluff body width & $778 \mathrm{~mm}$ \\
Bluff body height & $200 \mathrm{~mm}$ \\
Height of turbine's center from bluff body top surface & $80 \mathrm{~mm}$ \\
Distance of turbine's center from bluff body front edge & $100 \mathrm{~mm}$
\end{tabular}




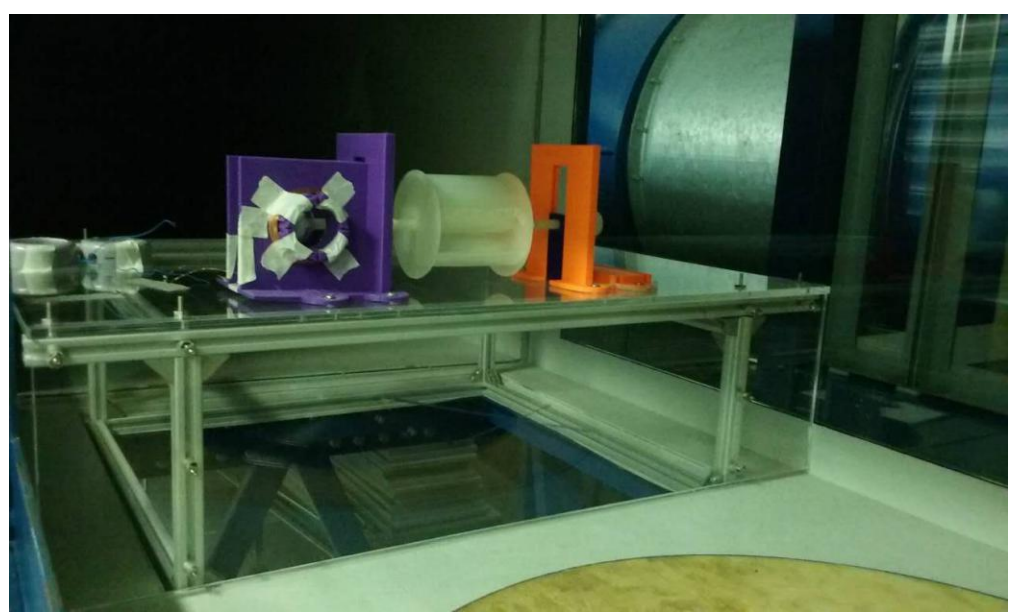

Fig.13 Energy harvesting system and step height platform installed in the wind tunnel test section.

\subsubsection{Fabrication of air-driven energy harvesters and auxiliary parts}

Additive manufacturing (AM) technologies have advanced dramatically in the past decades with advent of new materials, new processes and new technologies. Among them, 3-dimensional printing (3DP) is very competitive in terms of cost, speed and sales of related equipments [84].

3D printing offers a number of distinct advantages compared to other AM technologies. A few examples of them are summarized as follows:

- Due to ink jet principle and the fact that only physical phase change is involved in material melting and solidification process, 3D printing offers higher speed compared to other AM systems [85].

- Together with high speed advantage, lower material and equipment costs offer a cost advantage over other AM technologies [85]. 
- 3D printing printers can be office-friendly, and are often preferred in a design environment [85].

- 3D printed surfaces can be hand finished and painted [84].

- 3D printing shows decent accuracy with aver error values fluctuate in the order of $\pm 0.1 \mathrm{~mm}[84]$.

However, there are still some drawbacks associated with 3D printing technology, such as:

- The surface finish of 3D printing is poor compared to some of other AM processes [84][86].

- Currently, the materials available for 3D printing are still limited [84].

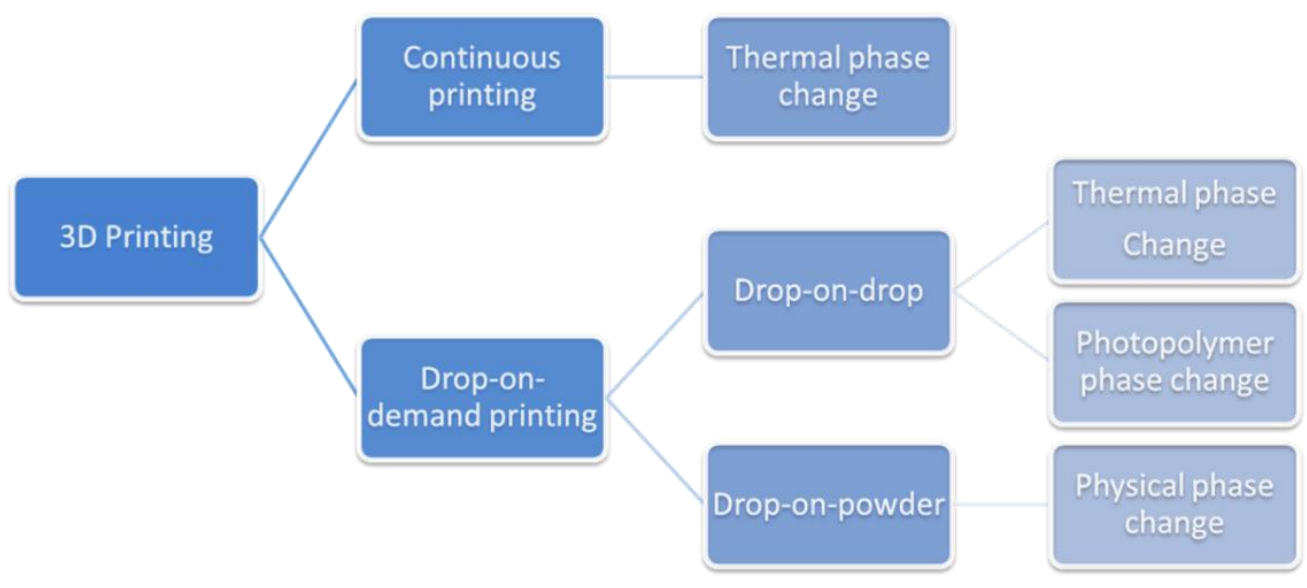

Fig.14 Classification of 3DP techniques. Adapted from Ref. [86].

Considering the cost of production, and suitability for design and prototyping processes, 3D printing is employed in this study for fabrication of energy harvester models and small auxiliary parts. There are different techniques available, and is 
shown in Fig.14. The technique employed in this study is continues printing based on thermal phase change.

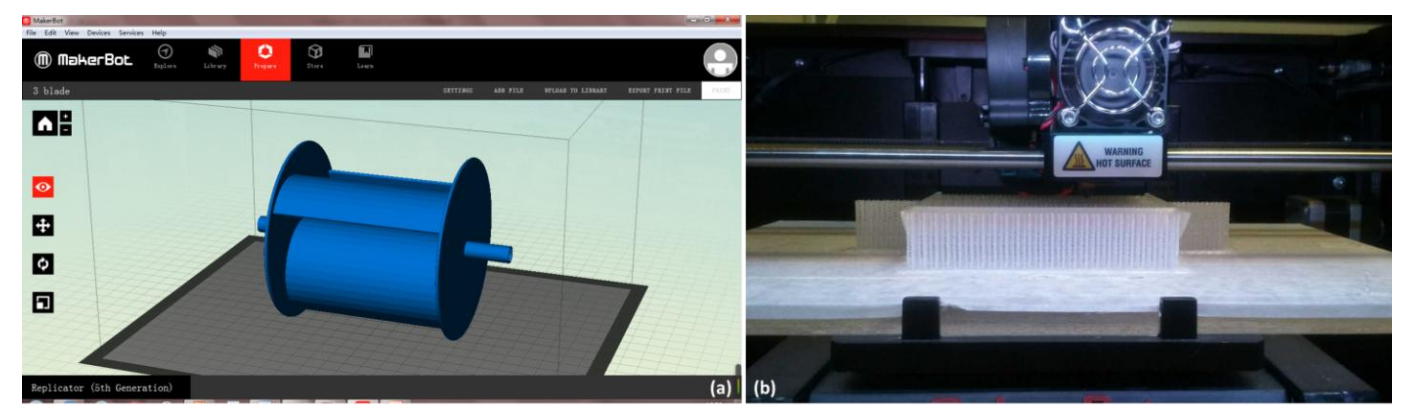

Fig.15 (a) Software preparing printer instruction file and (b) MakerBot Replicator 2 Desktop 3D Printer at work.

The 3D printer used in this study is MakerBot Replicator 2 Desktop 3D Printer, developed by MakerBot Industries [87]. It works based on Fused Filament Fabrication (FFF) method. Designed solid models are made from melted polylactic acid (PLA) filaments. The models designed by CAD softwares like SolidWorks are converted into STereoLithography (STL) format, and instruction files for the MakerBot Replicator 2 (Fig.15a) are sent to the machine via USB cable or SD card. Then the printer heats the PLA filament and extrudes it out through a nozzle while moving according to the instruction files. The solid object is made by layer-bylayer solidification of PLA (Fig.15b) [88].

The designed models are printed to the highest resolution available, and the printer settings are shown in Table 4. 
Table 4 Printer settings for energy harvester models and auxiliary parts fabrication.

\begin{tabular}{ll}
\hline Parameter & Value \\
\hline Infill & $100 \%$ \\
Layer height & $0.1 \mathrm{~mm}$ \\
Extruder temperature & $205^{\circ} \mathrm{C}$ \\
Extruder moving speed while extruding & $85 \mathrm{~mm} / \mathrm{s}$ \\
Extruder moving speed while traveling & $150 \mathrm{~mm} / \mathrm{s}$ \\
\hline
\end{tabular}

\subsubsection{Wind tunnel test}

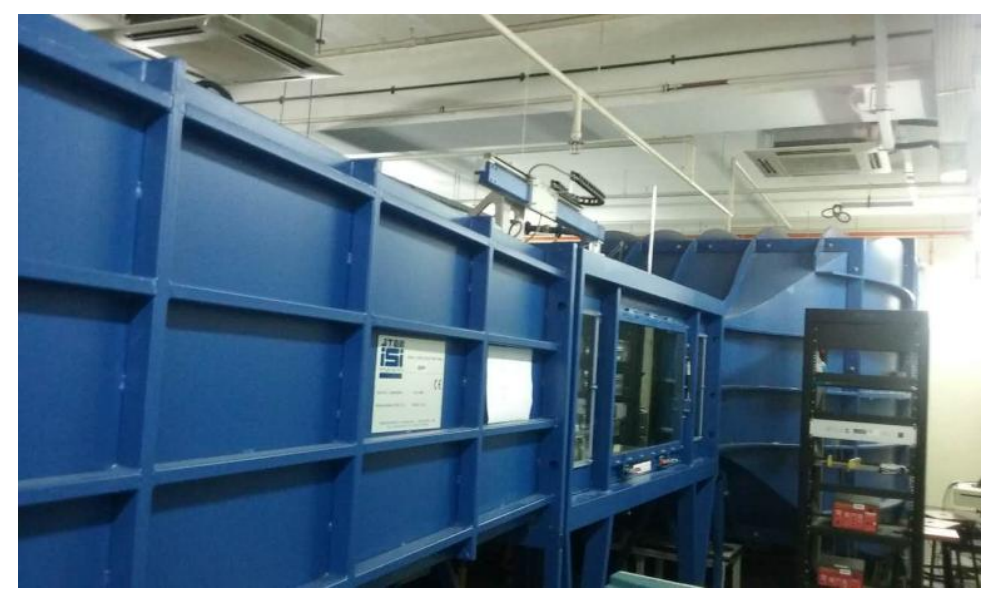

Fig.16 Closed loop wind tunnel at Nanyang Technological University.

The wind tunnel used is an AF6407 closed-loop wind tunnel with cooling of the circulating air at Nanyang Technological University. Force balance is not necessary for this study thus is not equipped in the tunnel test section. Wind tunnel test section has internal dimension of $780 \mathrm{~mm}(\mathrm{~W}) \times 720 \mathrm{~mm}(\mathrm{H}) \times 2000 \mathrm{~mm}(\mathrm{~L})$ (Fig.16). The wind turbine model together with all auxiliary parts and platform are installed in this test section. The wind turbine models then are to be tested at different Reynolds numbers in wind tunnel. 
The air flow rate is changed by controlling the fan speed in the wind tunnel. The control of wind tunnel fan speed is analog-based, which makes accurate adjustment of air flow rate practically difficult. The built-in anemometer of the wind tunnel is installed at the inlet of the test section. However, due to installation of the step height platform and energy harvester, blockage effect makes the air flow rate at the energy harvester different from that at the test section inlet. To mitigate theses effects, the air flow rate at the energy harvester site is measured by placing a Sentry ST372 IR thermo- Anemometer with build-in Infrared Thermometer at the vicinity of the harvester.

Meanwhile, the rotation speed of the wind energy harvester is monitored using Acez Portable Tachometer AI3030. This allows real time measurement of the energy harvester rotation speed in unit of revolutions per minute (RPM).

The electricity generated by electromagnetic induction is then measured by openloop method, and recorded by Tektronix TDS1012B Two Channel Digital Storage Oscilloscope. The wind energy harvester rotation induces electromotive force in the coil, which is electrically connected to the oscilloscope. The data obtained is then exported into USB drive for further analysis.

\subsubsection{Repeatability and reproducibility test}

At each air flow speed, emf is measured after the reading is stabilized. Emf is recorded three to four times at least at each air flow rate. As the rotation speed of 
the energy harvester is stabilized, the peak values of emf measurements are consistent.

It is very challenging to create the exactly same experimental setting. The air flow speed is altered by changing the fan speed of wind tunnel. But it is extremely difficult to control the fan speed so that the same air flow speed can be attained. Thus the experiment in the same range of air flow rate is repeated, and comparison result for benchmark model (harvester A, refer to Table 2) is shown in the figure below (Fig.17).

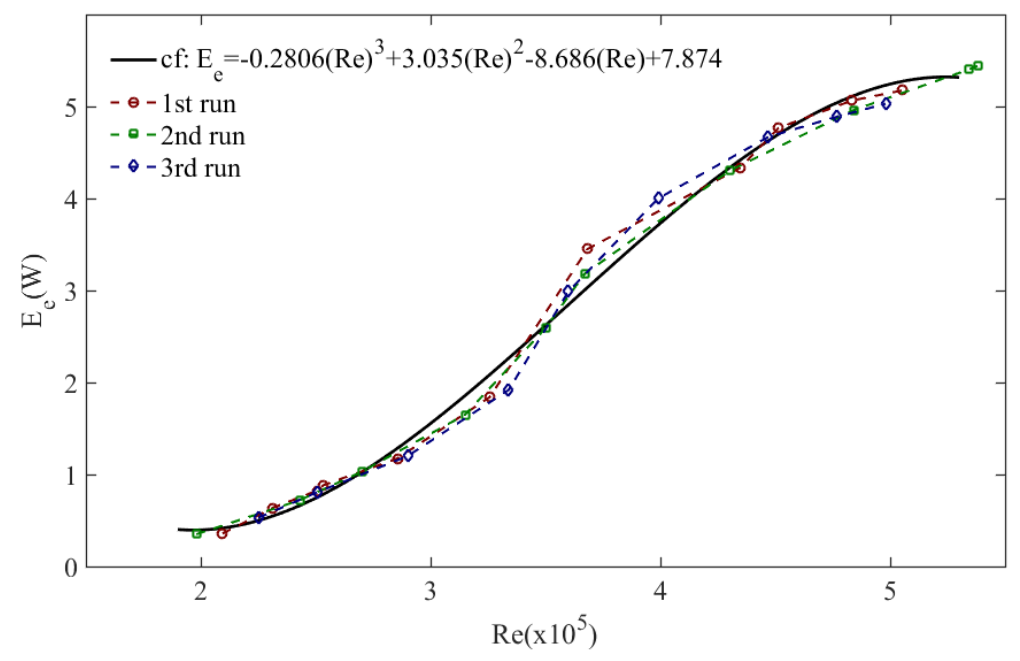

Fig.17 Comparison of measured results at different runs. 


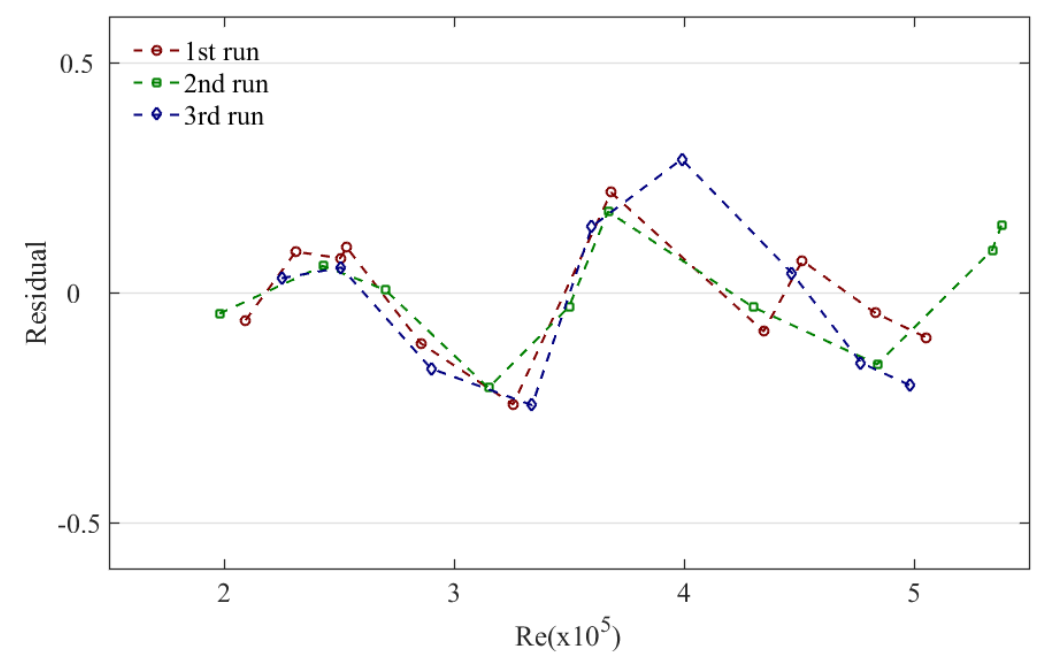

Fig.18 Residual of curve fitting results.

Curve fitting is done to capture the trend of variation of electrical power output with Reynolds number. The figure below shows that the residuals (Fig.18) and relative errors (Fig.19) are relatively small. These results indicate the measured emf values possess good repeatability and reproducibility.

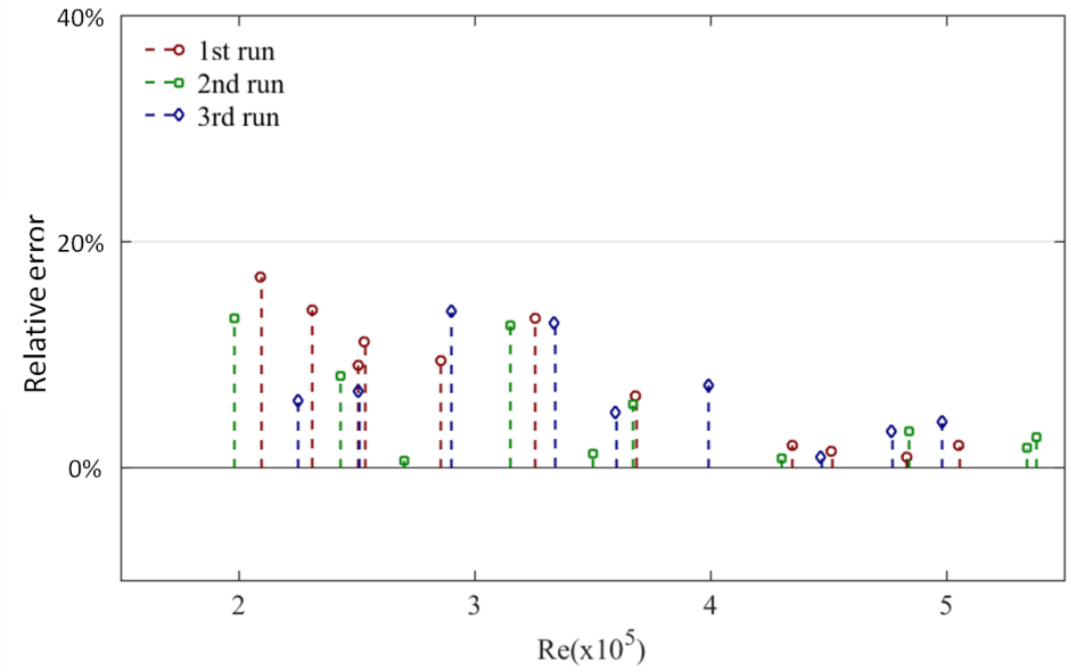

Fig.19 Relative errors in experimentally measured results. 


\subsection{Numerical work}

\subsubsection{Numerical model description}

In the experimental test, air flow rate is monitored at the vicinity of the energy harvester, thus the effects of blockage effect is mitigated. However, in numerical study, the air flow rate is set at the inlet of computational domain, thus the effects of blockage effect must be handled cautiously. Therefore, a blockage factor is introduced into determination of Reynolds number at the energy harvester model.

Blockage factor depends on the ratio of frontal areas of model (including the step height platform and accessories parts) and cross-sectional area of test section. For a closed loop wind tunnel as used in the experimental tests, the blockage factor $(f)$ is defined as [89]:

$f=\frac{1}{4}\left(\frac{\text { Model frontal area }}{\text { Test section cross }- \text { sectional area }}\right)$

Subsequently, the air flow speed needs to be corrected for blockage effect, and the corrected air flow speed $v_{c}$ is given by $v_{c}=v(1+f)$ where $v$ is the undisturbed air flow speed set at the inlet of the computational domain [90]. The Reynolds numbers used in numerical study are determined from this corrected air flow speed $v_{c}$.

The test section cross-sectional area is constant, which is $778 \mathrm{~mm} \times 720 \mathrm{~mm}=$ $560160 \mathrm{~mm}^{2}$. The approximate combined frontal area of step height platform and 
accessory parts is $(778 \mathrm{~mm} \times 200 \mathrm{~mm})+(20 \mathrm{~mm} \times 140 \mathrm{~mm}+110 \mathrm{~mm} \times$ $110 \mathrm{~mm})=170500 \mathrm{~mm}^{2}$. The frontal areas of the energy harvesters and corresponding blockage factors are shown in Table 5.

Table 5 Frontal areas and corresponding blockage factors of energy harvesters investigated in preliminary parametric study.

\begin{tabular}{|c|c|c|}
\hline Harvester & Frontal area $\left(\mathrm{mm}^{2}\right)$ & Blockage factor $(f)$ \\
\hline A & 10000 & 0.0805 \\
\hline B & 10000 & 0.0805 \\
\hline $\mathrm{C}$ & 10000 & 0.0805 \\
\hline $\mathrm{D}$ & 4444 & 0.0781 \\
\hline $\mathrm{E}$ & 17778 & 0.0840 \\
\hline $\mathrm{F}$ & 5000 & 0.0783 \\
\hline G & 5000 & 0.0783 \\
\hline $\mathrm{H}$ & 10000 & 0.0805 \\
\hline I & 10000 & 0.0805 \\
\hline $\mathrm{J}$ & 10000 & 0.0805 \\
\hline
\end{tabular}

All numerical works have been performed with general purpose commercial Computational Fluid Dynamics (CFD) software, ANSYS CFX 16.1 and 17.0, 
which are integrated in ANSYS Workbench. The set of equations solved by ANSYS CFX are unsteady Navier-Stokes equations in their conservation form. The flow of an incompressible Newtonian fluid governed by the Navier-Stokes equations can be written in dimensionless form as follows:

$\frac{\partial v}{\partial t}+(v \cdot \nabla) v=-\nabla p+\frac{1}{R e} \Delta v$

$\nabla \cdot v=0$

where $v$ is the velocity, $p$ is the static pressure, $t$ is the time, and $R e$ is the Reynolds number. ANSYS CFX is used to solve these equations based on the finite volume technique.

The solid geometries of the energy harvesters are designed and created with SolidWorks, and imported into ANSYS Workbench. The geometric characteristics of the energy harvesters are the same as those tested. The geometric characteristic parameters of the energy harvesters can be found in Appendix. The computational domain is set according to the dimensions of the wind tunnel, but only half of the experimental setup is modeled due to the vertical symmetry. This leads to considerable saving of elements and significant reduction of computational time. Note that as the energy harvester is installed on a step height, this step height is included in computational domain as well. The illustration of the computational domain is shown in Fig.20. At the velocity inlet, the distribution of velocity is set uniform. At the pressure outlet, it is set as ambient pressure. The no slip boundary condition is applied to the energy harvester surfaces. There were three sub-domains in the whole computational domain: the 'Wind tunnel sub-domain' consists of two 
stationary domains and the rotating 'Rotor sub-domain'. The cylindrical surface between the two sub-domains is set as an interface in order to ensure the continuity of the flow (Interface 2). Another interface is set within the 'Wind tunnel subdomain', but it has no physical meaning (Interface 1). The purpose of Interface 1 is to assist gradual increment of mesh density at locations closer to the energy harvester surfaces. The details of ANSYS CFX settings used in this study can be found in Table 6.
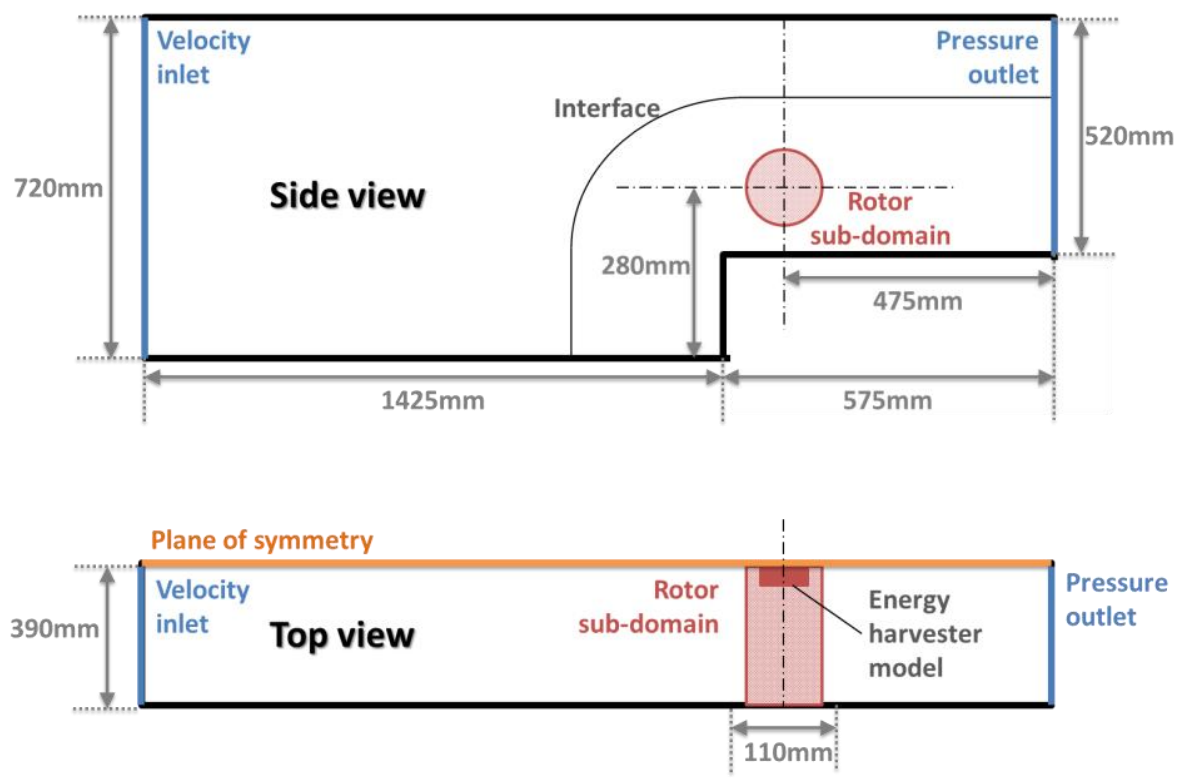

Fig.20 Side and top views of computational domain.

Table 6 ANSYS CFX settings for simulating the experiment.

Description

Computational domain size

Mesh topology
Value or setting

$$
390 \mathrm{~mm}(\mathrm{~W}) \times 720 \mathrm{~mm}(\mathrm{H})
$$$$
\times 2000 \mathrm{~mm}(\mathrm{~L})
$$

$3 \mathrm{D}$, symmetry at vertical plane 

wall functions and low-Re formulation

The 'Rotor sub-domain' is used to simulate the rotation of the energy harvester, and thus characterized by a moving mesh. When the dynamic performance of the energy harvester is evaluated, the 'Rotor sub-domain' is set to rotate at the same 
angular velocity as that of the energy harvester. As the energy harvester is enclosed in the 'Rotor sub-domain', the interface surface also serves to control the mesh size be denser as closer to the energy harvester surfaces. The gradual increment in mesh density towards 'Rotor sub-domain' can be clearly seen in Fig.21.

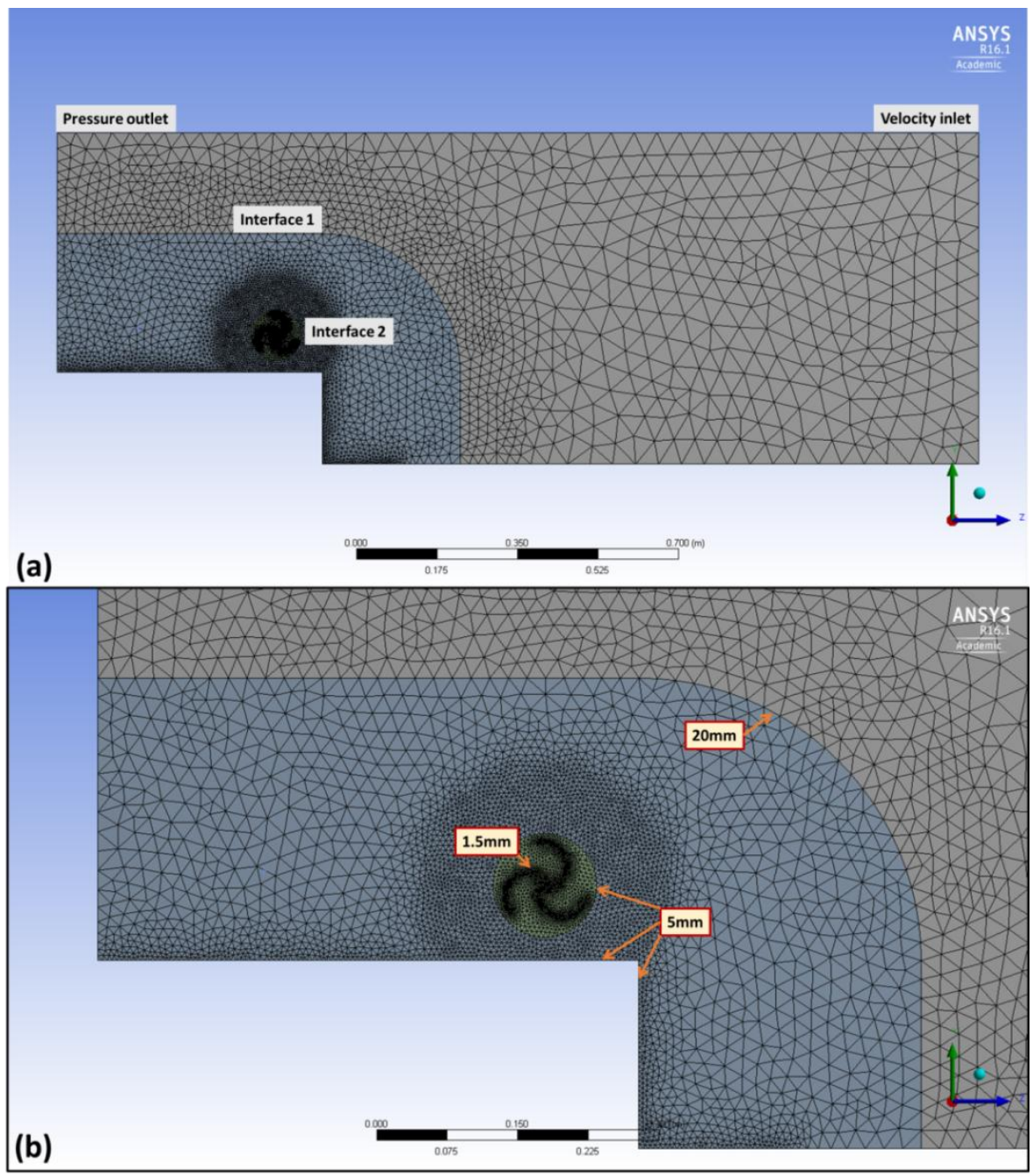

Fig.21 View of meshed situation for (a) whole computational domain and (b) closer view of 'Rotor sub-domain' containing the energy harvester. 
Grid independence study is conducted first to determine the final mesh size selected in the present simulations. The initial mesh size is set according to the following rules:

- The edge length of elements at the harvester surfaces is set to $2 \mathrm{~mm}$, which corresponds to a blade thickness and $2 \%$ of a turbine diameter.

- The interface between rotating and stationary domains (Interface 2) has an element edge length of $8 \mathrm{~mm}$, which is $1 / 10$ of the distance between the harvester shaft and the step height top surface. The elements along step height platform surfaces also have the same edge length as Interface 2.

- The interface between two stationary domains (Interface 1) has an element edge length of $25 \mathrm{~mm}$, which is half of the maximum element edge length.

Table 7 Details for meshes with different levels of fineness.

\begin{tabular}{|c|c|c|c|}
\hline \multirow{2}{*}{ Description } & \multicolumn{3}{|c|}{ Mesh fineness } \\
\hline & Coarse & Medium & Fine \\
\hline $\begin{array}{l}\text { Energy harvester surfaces } \\
\text { element face size }\end{array}$ & $2 \mathrm{~mm}$ & $1.5 \mathrm{~mm}$ & $1 \mathrm{~mm}$ \\
\hline $\begin{array}{l}\text { Interface face size between } \\
\text { stationary and rotating sub- } \\
\text { domains }\end{array}$ & $10 \mathrm{~mm}$ & $5 \mathrm{~mm}$ & $3 \mathrm{~mm}$ \\
\hline $\begin{array}{l}\text { Interface face size within } \\
\text { stationary sub-domain }\end{array}$ & $25 \mathrm{~mm}$ & $20 \mathrm{~mm}$ & $10 \mathrm{~mm}$ \\
\hline Maximum cell face size & $50 \mathrm{~mm}$ & $50 \mathrm{~mm}$ & $50 \mathrm{~mm}$ \\
\hline
\end{tabular}


Having a denser mesh is more desirable for capturing flow properties fluctuations. However, a very fine mesh or dense mesh requires significantly larger computational resources and time. As the level of fineness is further increased, the refinement effect on numerical results diminishes. And beyond a certain level of fineness, vast computational resources are spent on negligible refinement effect. The balance between the two is found by conducting this grid independence study, and the results can be seen in Fig.22. The meshing details of different levels of fineness can be found in Table 7 . It can be seen that variation in torque is much larger with coarse mesh, as compared to medium and fine meshes. The difference between medium and fine meshes is not significant. In order to save computational resources, mesh with medium level of fineness is chosen.

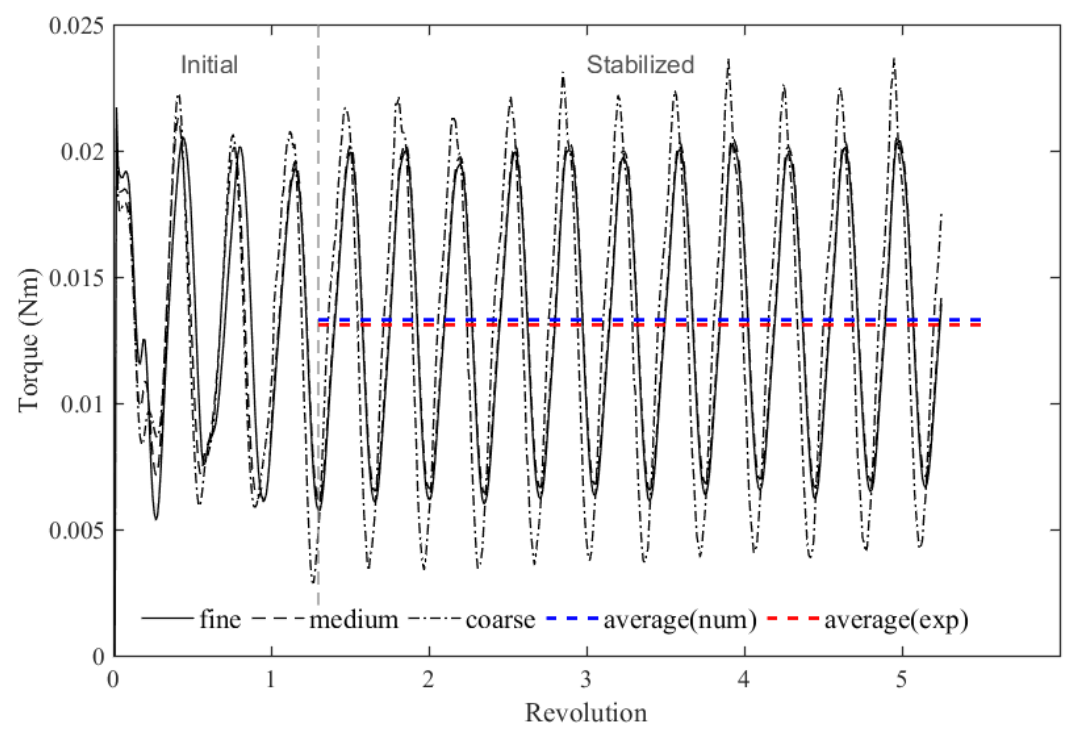

Fig.22 Variation of torque value with different levels of mesh fineness. ('num' denotes numerically predicted results and 'exp' denotes experimental results). 
For validation, the experimentally derived torque $T$ is compared with the numerically calculated one. Instead of using a torque transducer to directly measure the shaft torque, alternative method is adopted based on the basic relationship which defines torque as:

$T=J \frac{d \omega}{d t}$

where $J$ is the moment of inertia of the rotor about the axis of rotation and $d \omega / d t$ is the angular acceleration.

The moment of inertia, $J$, about the axis of rotation which is the turbine axis, is given by:

$J=\rho_{\text {turbine }} \int\left(x^{2}+y^{2}\right) d z d x d y$

where $\rho_{\text {turbine }}$ is the density of harvester material, the $z$-axis is the turbine axis, $y$ axis is in the radial direction and $x$ is in the direction of the oncoming wind. $\rho_{\text {turbine }}=1.21 \mathrm{~g} \cdot \mathrm{cm}^{-3}$ is the density of 3D printing feeding material, polylactic acid (PLA). The integration is over the turbine volume. And $J$ is determined using Solidworks by using its built-in mass properties function.

The angular acceleration $d \omega / d t$ is estimated by $\Delta \omega / \Delta t$ using the wind tunnel experimentation. The test is done by holding the turbine stationary initially and allowing it to free rotate and accelerate after the air flow speed in the wind tunnel is stabilized. Tachometer is used to constantly monitor the angular speed of the energy harvester until the angular velocity stabilized eventually. The stabilized 
angular speed $(\Delta \omega)$ and the time taken for the angular speed to stabilize $(\Delta t)$ are measured and used to estimate the angular acceleration.

$T \approx J \frac{\Delta \omega}{\Delta t}$

It is to be noted that only models with low cut-in Reynolds numbers are compared as they are able to self-start rotation at low air flow speed. Therefore, two of energy harvester models are used for validating numerical results (Fig.23). The design details about these two energy harvester models can be found in Table 8 . It can be seen general agreement of torque on harvester blades value is achieved between measurements and numerically predicted results. A few wind tunnel tests are conducted on the two energy harvester models for validation purpose, and the resultant torque values match the numerically predicted trends. However, it has to be noted that the numerical model constantly overestimate the value of torque. Although blockage effect is taken into consideration in the determination of inlet velocity process, the corrected air flow speed is still lower than the actual value. Hence, it must be noted that this overestimation of torque will lead to subsequent overestimation of torque and power coefficients. 


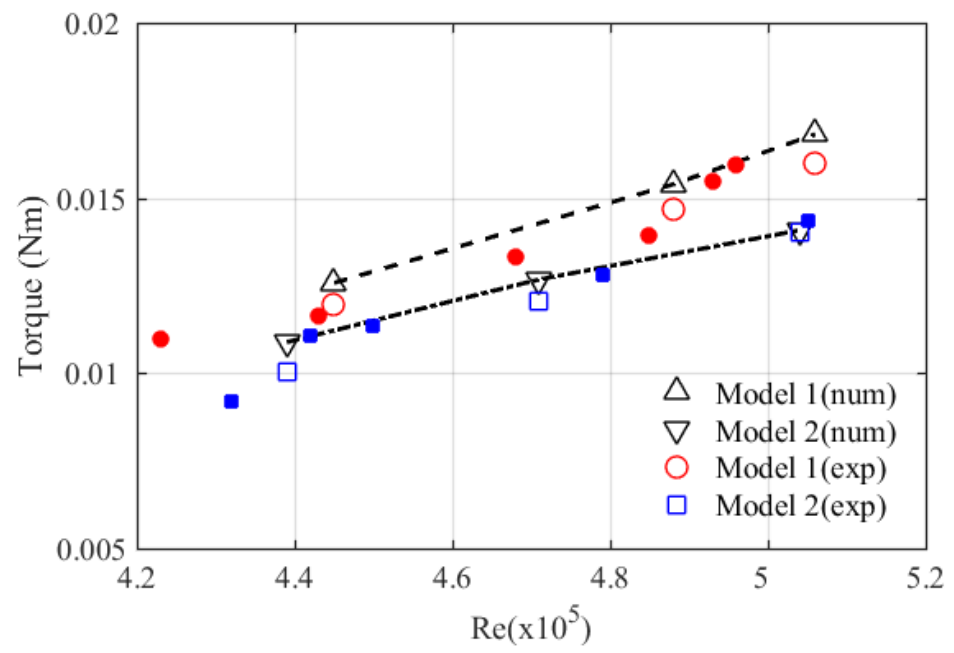

Fig.23 Validation of numerical study results. (Specification of Model 1 and 2 can be found in Table 8.Pair of empty markers from numerical and experimental results share the same conditions. 'num' denotes numerically predicted results and 'exp' denotes experimental results)

Table 8 Design parameters details of energy harvester models used for validation.

Design parameter

Model 1

Model 2

\begin{tabular}{lll}
\hline Number of blades & 3 & 3 \\
Harvester length & $100 \mathrm{~mm}$ & $100 \mathrm{~mm}$ \\
Blade thickness & $2 \mathrm{~mm}$ & $2 \mathrm{~mm}$ \\
End plate presence & Yes & No \\
Central shaft presence & No & Yes \\
Harvester orientation & 'clockwise' in z direction & 'clockwise' in z \\
& & direction \\
Blockage factor & 0.0805 & 0.0805 \\
\hline
\end{tabular}




\subsubsection{Static performance of air-driven energy harvester}

Self-starting capability is one of the important considerations when evaluating the performance of air-driven energy harvester. An air-driven energy harvester with excellent self-starting capability is able to start to rotate at relatively lower Reynolds number. This means it should have a low cut-in Reynolds number $\left(R e_{c}\right)$, which is the minimum Reynolds number that the energy harvester can self-start rotation without any additional or external triggering force in experiments. Hence, it is important to study the static performance of wind energy harvesters and examine whether the energy harvester possesses self-starting capability.

Numerical approach can be adopted to study static performance of air-driven energy harvesters. In order to do so, the static torque needs to be evaluated at different rotor angles $(\theta)$, where rotor angle is defined as the angle between the turbine blade and the oncoming air flow direction (Fig.24).

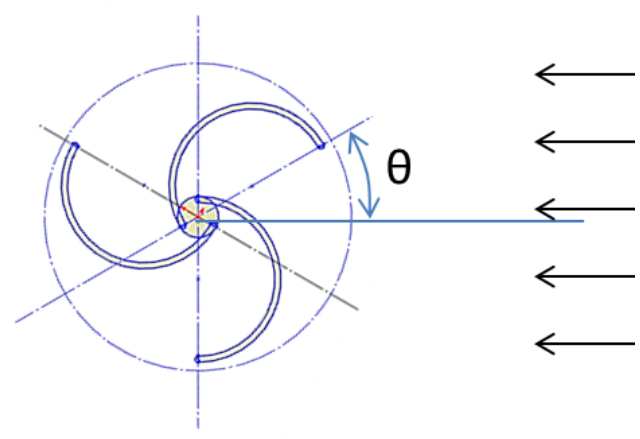

Fig.24 Illustration of rotor angle $\theta$ 
Static torque $\left(T_{s}\right)$ is the torque exerted on the energy harvester blades while the harvester is held stationary. Static torque coefficient $\left(C_{T s}\right)$ can be evaluated with Eq. (16):

$C_{T s}=\frac{T_{s}}{0.5 \rho A R\left(\mu R e / \rho D_{h}\right)^{2}}$

where the static torque on turbine blades can be monitored in numerical study. Low static torque coefficients (negative values or values close to zero) imply very poor self-starting capability, while larger static torque coefficients are plausible.

The torque generated by moving air mass on energy harvester turbine blade surfaces is monitored while the harvester is fixed at certain rotor angle, and thus static torque coefficient can be evaluated. By plotting static torque coefficient versus rotor angle for different energy harvester models, the self-starting capabilities of these energy harvester models can be represented and compared. Also, the static torque coefficient versus rotor angles graphs can be obtained at different Reynolds numbers; hence the effect of Reynolds number on the static performance of wind energy harvesters can be investigated.

\subsubsection{Dynamic performance of air-driven energy harvesters}

To study dynamic performance of air-driven energy harvesters, and thus to obtain the power coefficients of these energy harvesters, dynamic torque coefficient $\left(C_{T d}\right)$ should be evaluated.

Dynamic torque coefficient can be calculated by Eq. ( 17): 
$C_{T d}=\frac{T}{0.5 \rho A R\left(\mu R e / \rho D_{h}\right)^{2}}$

where $\mathrm{T}$ is the torque on wind turbine blade surfaces exerted by moving air mass while the air-driven energy harvester is rotating.

It must be noted that power coefficient $\left(C_{P}\right)$ which is defined as the ratio of mechanical power possessed by the energy harvester $\left(E_{\text {harvester }}\right)$ to the power available in moving air $\left(E_{a}\right)$, which indicates how efficient the energy harvester is. But overall energy conversion efficiency is the product of harvester power coefficient and efficiency of electromagnetic converter. This power coefficient is also related to torque coefficient by Eq. ( $\mathbf{1 8})$ :

$C_{P}=\frac{P}{0.5 \rho A R\left(\mu R e / \rho D_{h}\right)^{3}}=\frac{T}{0.5 \rho A R\left(\mu R e / \rho D_{h}\right)^{2}} \frac{\omega R}{\left(\mu R e / \rho D_{h}\right)}=C_{T d} \lambda$

where $\lambda$ is tip speed ratio.

To study the dynamic performance of miniature air-driven energy harvester as discussed, the dynamic torque coefficients can be evaluated at different tip speed ratios $(\mathrm{TSR}, \lambda)$ by changing the rotation speed of the harvester under constant Reynolds number. Therefore, the performance characteristic curves of air-driven energy harvesters can also be obtained. 


\section{Chapter 4. Results and Discussion}

\subsection{Preliminary parametric measurements}

Ten miniature harvesters driven by air flow are experimentally evaluated and compared. For comparison, an initial design of such energy harvester is chosen to be a benchmark model, i.e. harvester A. Critical design parameters are identified as:

- Number of the harvester blades, $\mathrm{N}$

- Size ratio (SR) characterizing the geometric size of the energy harvester. SR is defined as the ratio of the geometric size of the harvester being tested to that of the benchmark model.

- Aspect ratio of the harvester (AR). It is defined as the ratio of the harvester length to the diameter of the harvester end plate.

- The type of central part (a solid central shaft or an empty central gap).

- The presence or absence of end plates.

- The installation orientation of the harvester.

The effects of these parameters are investigated one at a time. The output electrical power and energy conversion efficiency are measured as critical performance index of each energy harvester, as oncoming air flow is varied.

\subsubsection{Performance of the benchmark model}

Here the performance of the benchmark energy harvester, i.e. harvester A is chosen as an example to illustrate detailed calculation and experimental measurements. 
Same analysis and measurements are conducted on other harvester models, as oncoming air flow velocity is varied. To make our studies more generalized, Reynolds number and the corresponding power available are determined by measuring the air flow velocity. The electrical power output is determined by using Eq. (6) with the maximum/peak voltage measured. The overall energy conversion efficiency is predicted by using Eq. (9) with the potential wind power available and the electrical power output obtained. Detailed calculations are shown below:

\section{$\underline{\text { Available power } \underline{E}_{a}}$}

(for example, at $R e=2.09 \times 10^{5}$ )

$$
\begin{gathered}
E_{a}=\frac{A\left(\mu R e / \mathcal{D}_{H}\right)^{3}}{2 \rho^{2}}=\frac{0.01 \times\left(1.983 \times 10^{-5} \times 2.0939 \times 10^{5} / 0.80\right)^{3}}{2(1.225)^{2}} \\
=0.466 \mathrm{Watts}
\end{gathered}
$$

\section{$\underline{\text { Output electrical power } \mathrm{E}_{\mathrm{e}}}$}

(measured $V_{\text {peak }}$ is $0.357 \mathrm{~V}$ at $R e=2.09 \times 10^{5}$ and resistance $R_{r}=94.8 \Omega$.)

$$
E_{e}=\frac{\xi_{\mathcal{N}}^{2}}{2 R_{r}}=\frac{\xi_{r m s}^{2}}{R_{r}}=\frac{V_{\text {peak }}^{2}}{2 R_{r}}=\frac{0.357^{2}}{2 \times 94.8}=0.0013 \text { Watts }
$$

\section{$\underline{\text { The overall energy conversion efficiency } \eta_{\text {overall }}}$}

$$
\eta_{\text {overall }}=\frac{E_{e}}{E_{a}}=\frac{0.0013}{0.466} \times 100 \%=0.279 \%
$$


The benchmark harvester, i.e. harvester A is experimentally observed to start to rotate at approximately $\operatorname{Re}=5.95 \times 10^{5}$. Self-rotation can be maintained, as Reynolds number is reduced to $R e=2.09 \times 10^{5}$. Thus harvester A is tested, as Re is increased from $2.0 \times 10^{5}$ to $5.0 \times 10^{5}$.
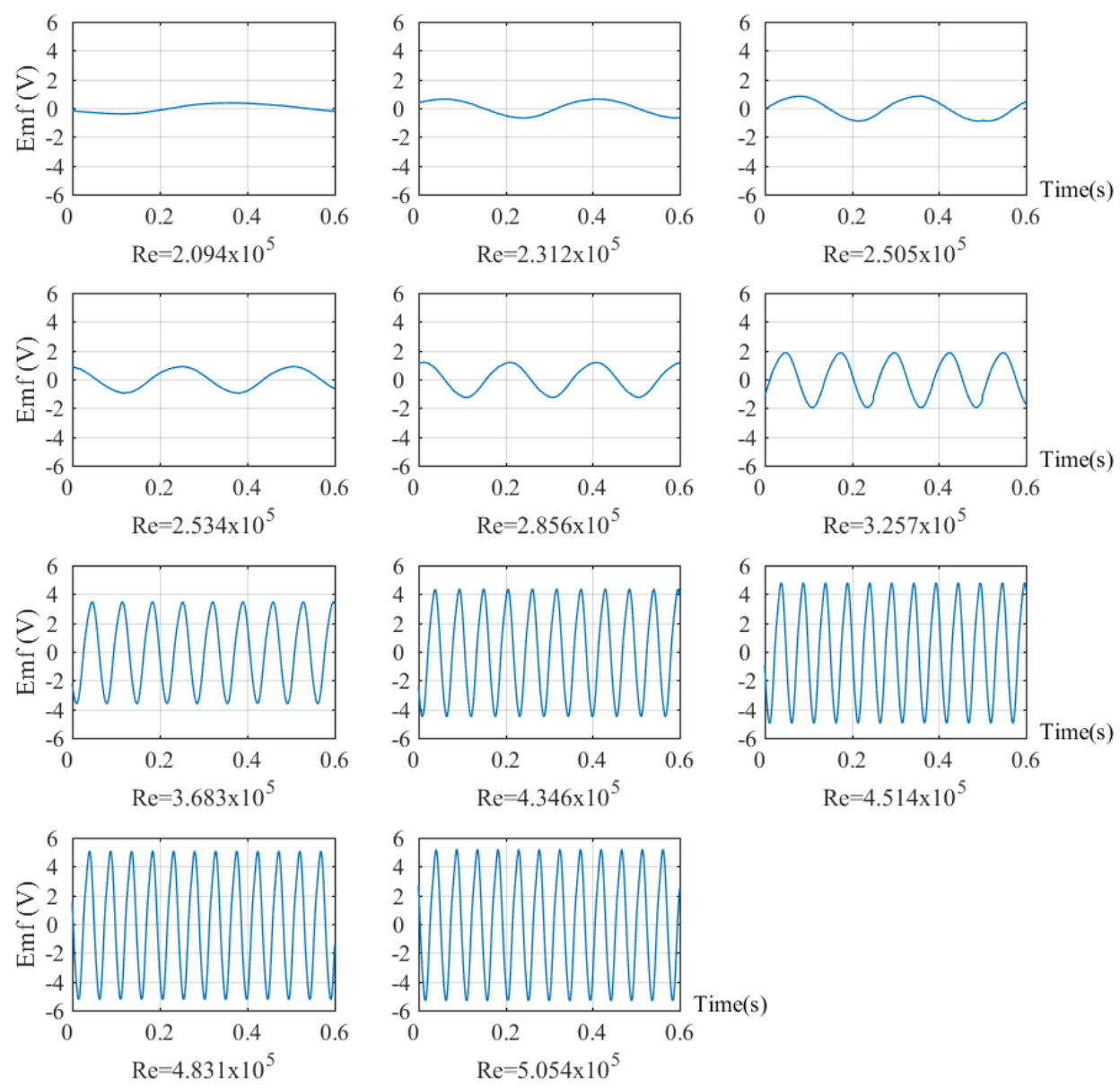

Fig.25 Time evolution of measured emf from harvester $A$ at various Reynolds numbers. 
Time evolution of the measured emf is shown in Fig 25.
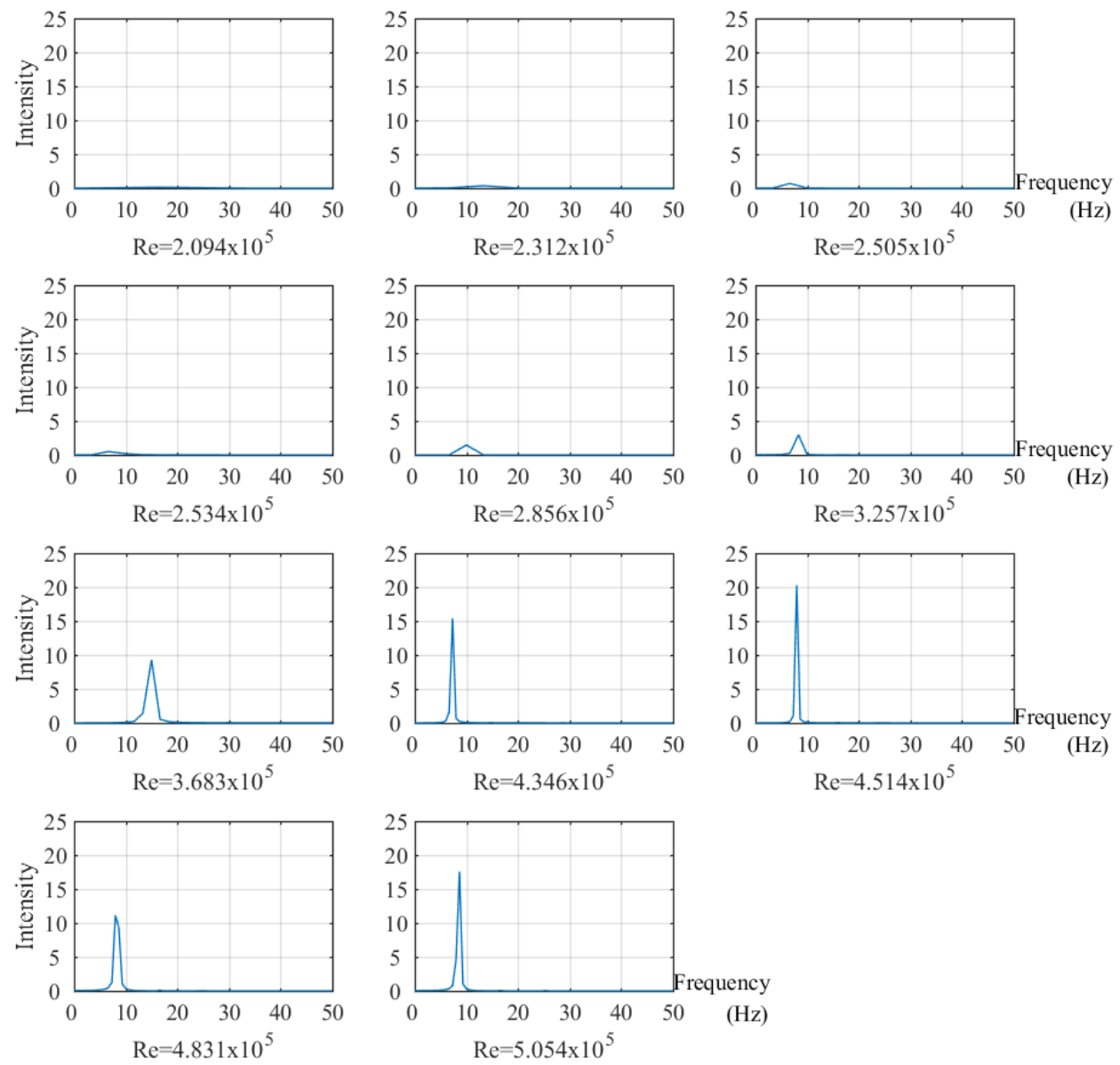

Fig.26 FFT plot of measured emf from harvester Model A at various Reynolds numbers. 

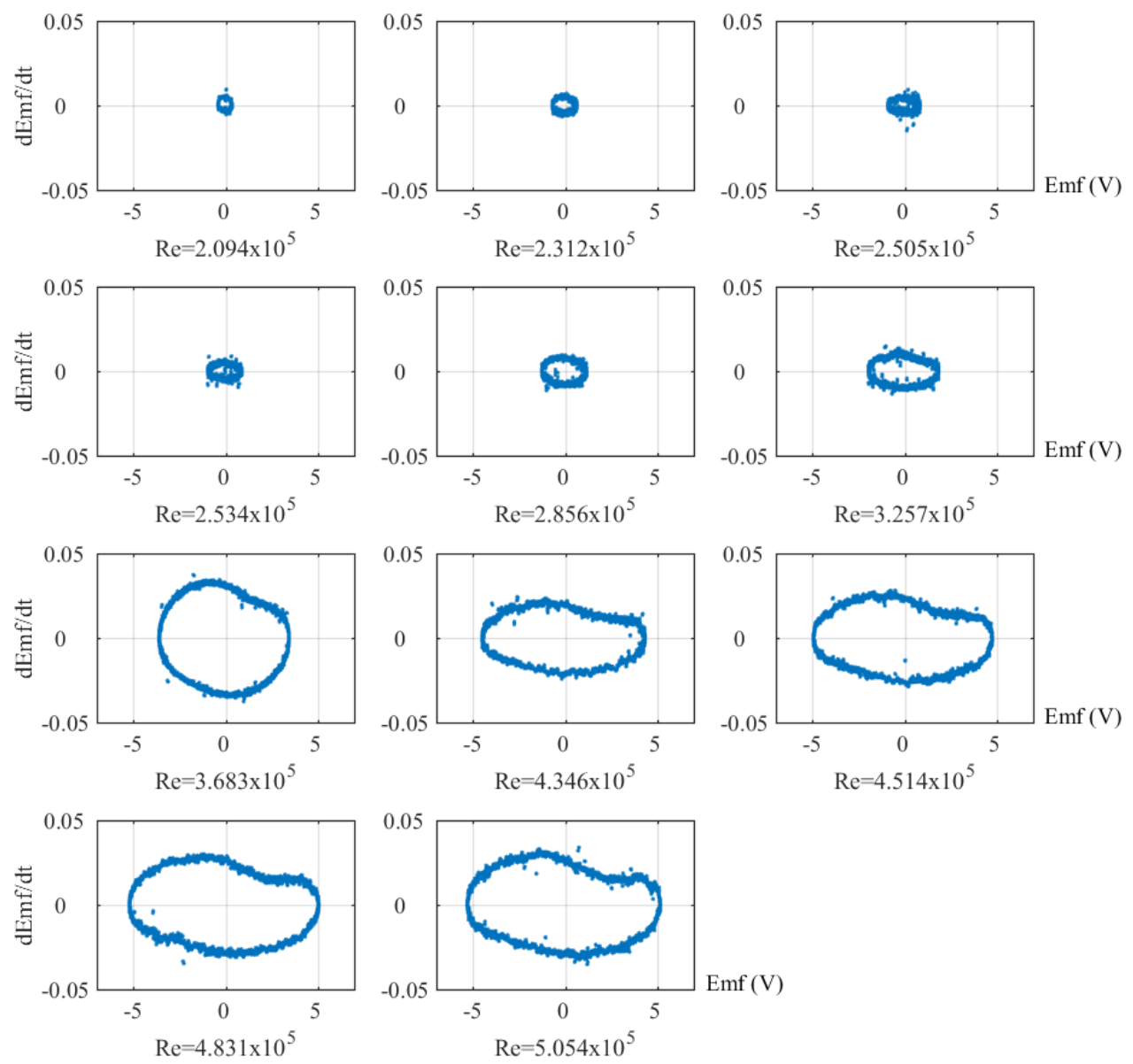

Fig.27 Phase plot of measured emf from harvester Model A at various Reynolds numbers.

It can be seen that the induced emf is oscillating at low frequency. However, the oscillation's frequency and amplitude are increased with increased Reynolds number until saturation is reached. The saturation of induced emf is most likely due to the mechanical friction between harvester shaft and its support. To gain insights on the oscillating emf, both frequency spectrum and phase plots of the measured emf are shown in Fig. 26 and 27. It can be seen from the frequency spectrum that the harvester is producing electric power at approximately $8 \mathrm{~Hz}$. The amplitude of 
measured emf is increased with increased Reynolds number. The non-circle shaped phase diagram as shown in Fig. 27 reveals that the energy harvesting system is a nonlinear one. The measured emf consists of multiple frequency oscillations. Similar measurements and analysis are conducted to other harvester/models. Detailed information can be found in Appendix B.
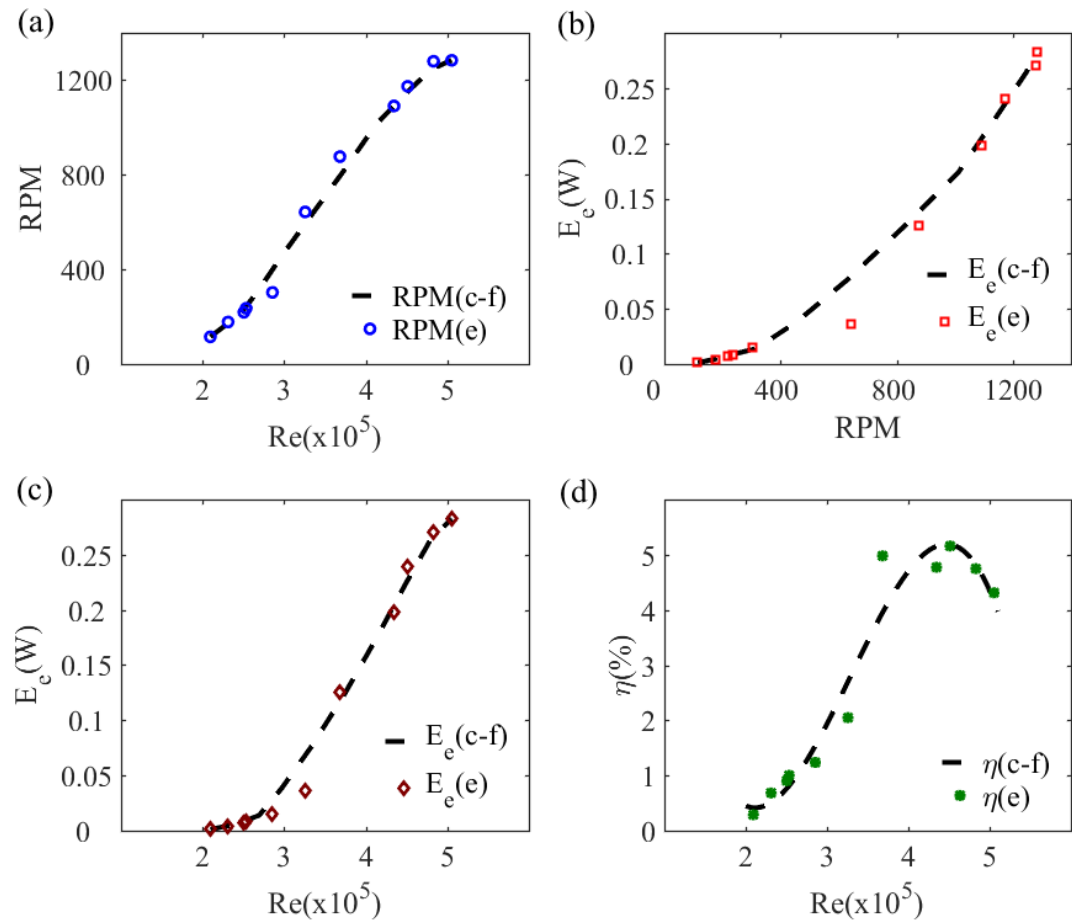

(d)

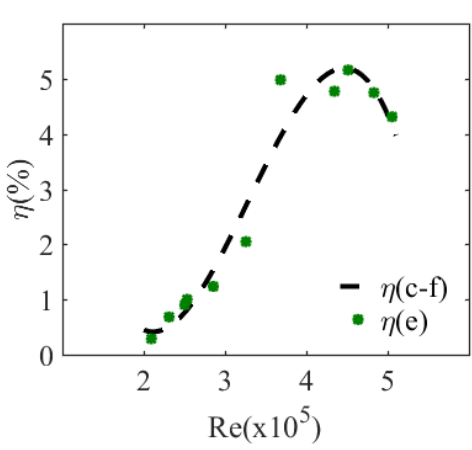

Fig.28 (a) RPM variation with Reynolds number. (b) Electrical power output varied with RPM. (c) Electrical power and (d) Overall energy conversion efficiency varied with Reynolds number for energy harvester Model A. (c-f denotes curve fitting and e denotes experimental results).

The rotation speed of the energy harvester A is measured, as Reynolds number is varied. Fig.28a shows the rotation speed in terms of revolutions per minute (RPM). It is apparent that at higher Reynolds number, higher RPM, larger induced emf and so more electrical power output are achieved as shown in Fig.28b and Fig.28c, 
respectively. However, the overall energy conversion efficiency $\eta$ is shown to be increased first then decreased as shown in Fig.28d. The maximum overall energy conversion efficiency achieved by this benchmark model is $\eta_{\text {overall } \max }=5.16 \%$ at $R e=4.514 \times 10^{5}$ approximately. This is due to the fact that at high Reynolds number, rotation speed of the harvester model is limited by friction between structures, and thus the electrical power output increases with a shallow gradient than $\mathrm{E}_{\mathrm{a}}$.

The electrical power harnessed from the oncoming air flow can be used for many purposes. For convenience, however, the energy harvester model is coupled with red and white light-emitting diodes (LEDs) for demonstration as shown in Fig.29. Here the Reynolds number is approximately $3.0 \times 10^{5}$, and six LED lights connected in series are powered. The application indicated that the proposed miniature energy harvester model is able to provide a simple, low-cost but robust platform to harness mechanical energy from moving air flow to generate electricity. 


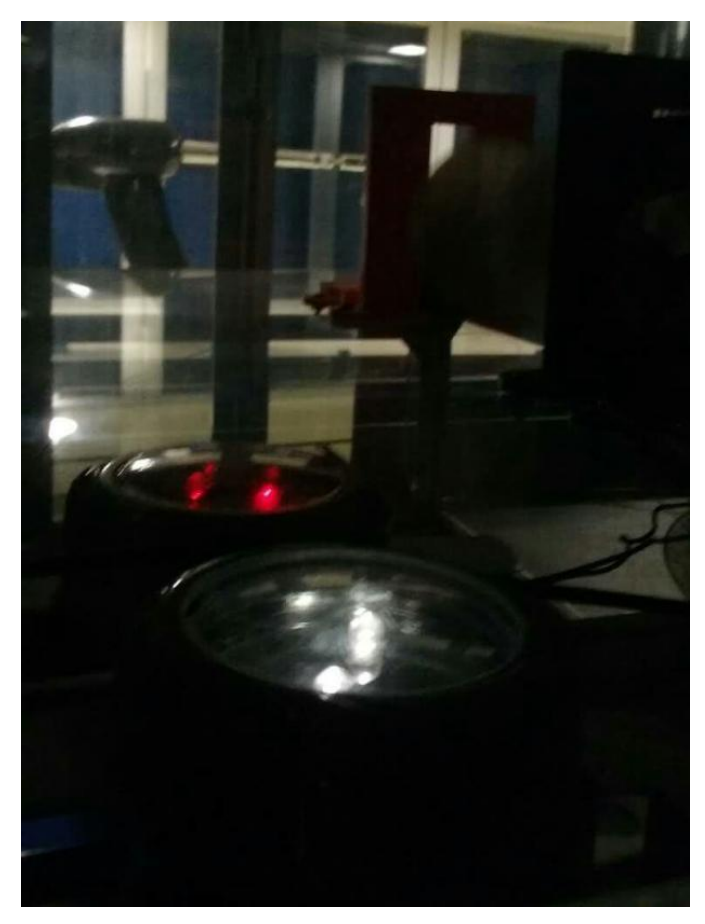

\section{Fig.29 Red and white LED lights powered by the energy harvester A.}

To study the static performance of the energy harvester A, static torque coefficients at different rotor angles are numerically determined at different Reynolds numbers as shown in Fig.30. It can be seen that increasing Reynolds number will increase the static torque coefficient, and make the harvester more likely to self-start rotation. However, it is also noticed that the extent of the enhancement effect of increasing Reynolds number is not significant. Note that large and positive static torque coefficient is preferred, since a static torque coefficient being negative or close to zero indicates the harvester is more unlikely to self-start rotation. It is also observed from Fig.30 that there are both positive and negative values of static torque coefficient for harvester A. as the rotor angle is in the ranges of $25^{\circ}-50^{\circ}$, $145^{\circ}-170^{\circ}$, and $265^{\circ}-290^{\circ}$, the static torque coefficients are negative. This means in these ranges of rotor angles, the harvester is not likely to self-start rotation. The 
maximum values of static torque coefficient take place at $100^{\circ}, 220^{\circ}$ and $340^{\circ}$, while the minimum static torque coefficients take place at rotor angles of $40^{\circ}, 160^{\circ}$ and $280^{\circ}$. These suggest the favorable self-starting positions (maximum $C_{T s}$ ) and the unfavorable positions (minimum $C_{T s}$ ) of harvester $\mathrm{A}$.

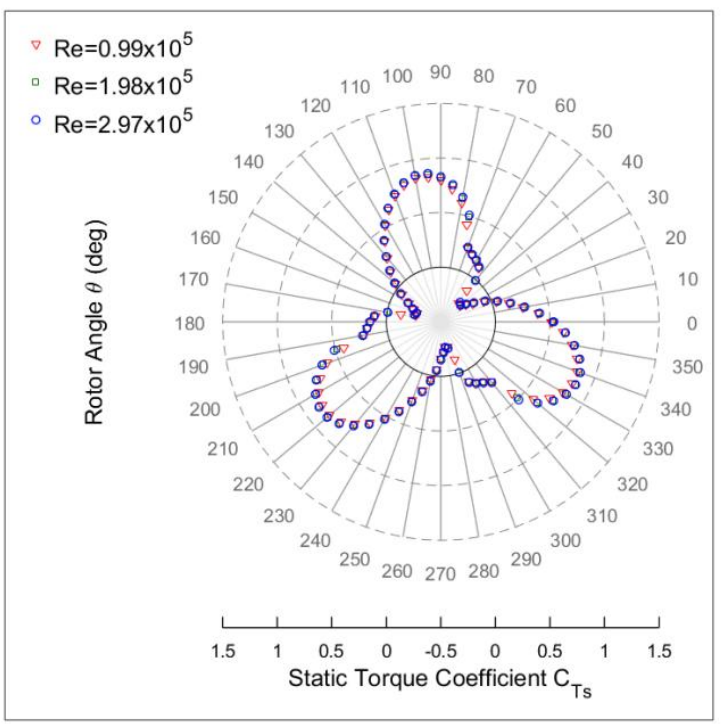

Fig.30 Variation of static torque coefficients at different rotor angles at different Reynolds numbers for harvester $A$. 


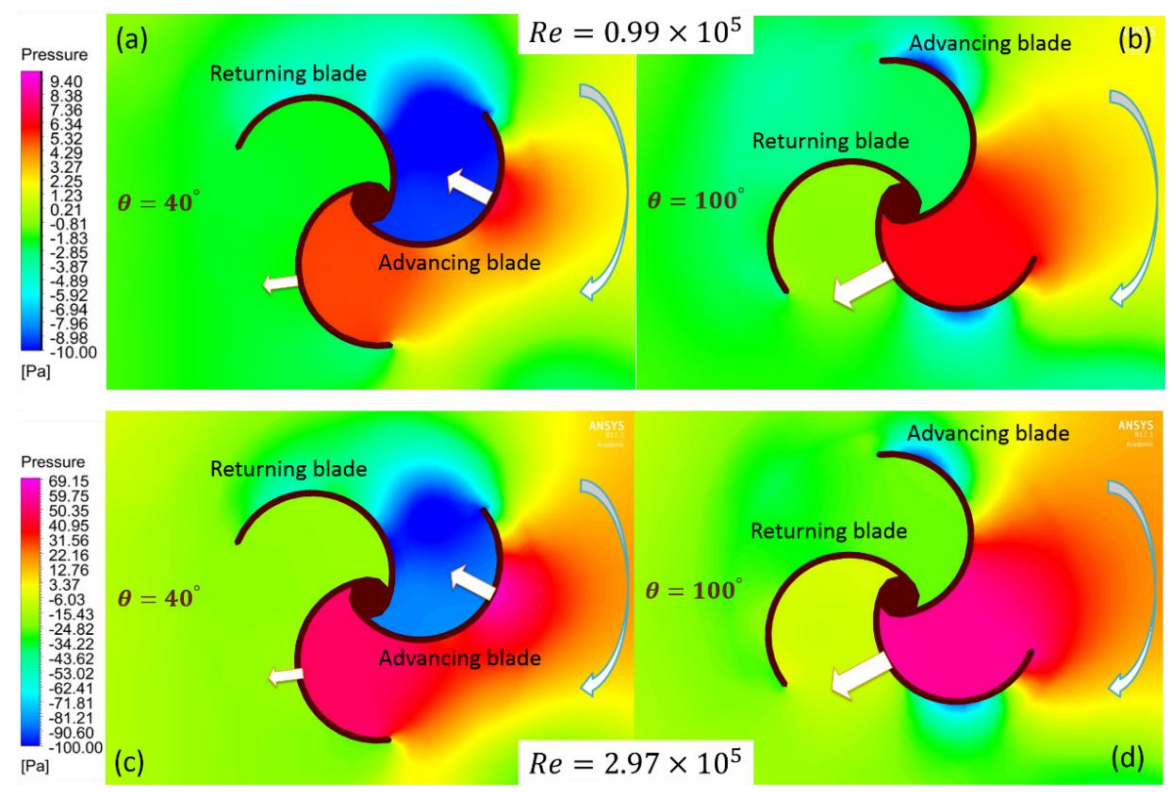

Fig.31 Pressure distribution patterns at $\operatorname{Re}=0.99 \times 10^{5}$ when (a) $\theta=40^{\circ}$, (b) $\theta=100^{\circ}$ and at $\operatorname{Re}=2.97 \times 10^{5}$ when (c) $\theta=40^{\circ}$, (d) $\theta=100^{\circ}$ for energy harvester $A$.

The static performance of the energy harvester is mainly determined by the pressure distribution in the energy harvester region. The pressure distribution pattern is jointly determined by the geometric structure of the energy harvester and the relative position between the energy harvester blades and the oncoming air flow (rotor angle). The pressure distribution patterns of the harvester corresponding to maximum and minimum static torque coefficient at different Reynolds numbers are shown in Fig.31. As the static torque coefficient is positive, the pressure is built up at the concave side of the blades (Fig.31b and Fig.31d) and produces a torque in favor of rotation direction. In contrast, when high-pressure region is at the convex side of the blades (Fig.31a and Fig.31c), a torque is generated in the direction against rotation and the resultant static torque coefficient is even smaller. It is observed that increasing Reynolds number can increase the static torque coefficient but not significantly, and the harvester rotor angle is the dominant factor. This is 
confirmed in Fig.31, increasing Reynolds number leads to a greater pressure difference between two sides of the harvester blade, but pressure distribution near the harvester at $R e=2.97 \times 10^{5}$ exhibits a similar pattern as that at lower $R e=0.99 \times 10^{5}$. As increasing Reynolds number does not affect the location of the high-pressure region, the static torque coefficient is not greatly varied. Therefore, the geometric structure of the energy harvester and its relative position with oncoming air flow are shown to determine the self-starting capability of the harvester.

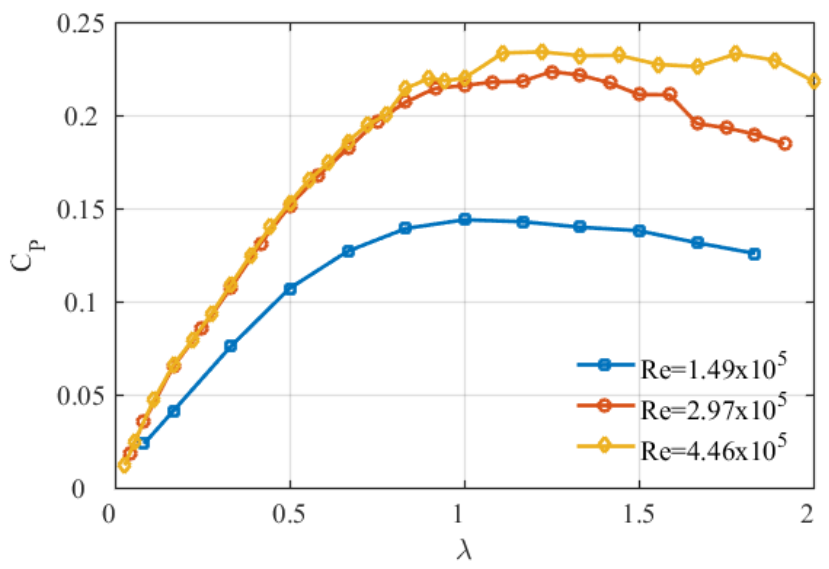

Fig.32 Performance characteristic curves for harvester $A$ at $\operatorname{Re}=1.49 \times 10^{5}$, $\operatorname{Re}=2.97 \times 10^{5}$ and $\operatorname{Re}=4.46 \times 10^{5}$.

The dynamic performance of the harvester A is also numerically studied. The performance characteristic curves are obtained for different Reynolds numbers, as shown in Fig.32. It can be seen that as the Reynolds number is increased, the power coefficient is increased as well, indicating the harvester can harness energy from moving air mass more efficiently. At a given Reynolds number, the power coefficient is increased with increased tip speed ratio first, then decreases. This is 
most likely due to increasing rotation speed, the energy harvester gradually harnesses energy more efficiently. In the regime of descending power coefficient, the harvester is rotating faster than the speed at which the air flows and momentum is transferred from the harvester blades to the air flow. This is not likely to take place in practice for a miniature harvester with a small diameter without any additional external driving forces. Thus more attention is paid to the regime of increasing power coefficient. Analyzing the pressure distribution at different Reynolds number and different tip speed ratios reveals more interesting findings.

Fig.33 illustrates the pressure distributions around the harvester $\mathrm{A}$ at different Reynolds numbers and different tip speed ratios. Fig.33a corresponds to the tip speed ratio at which the harvester can experimentally sustain rotation at $R e=1.49 \times 10^{5}$, and it can be seen the pressure is well concentrated near the concave side of the blade. The energy harvester is set to rotate at a faster rotation speed in Fig.33b, the pressure distribution pattern is greatly altered. Unfortunately, this indicates that there is insufficient time for high-pressure region to develop at desired location and this makes it unrealistic to rotate in practice. Increasing Reynolds number (Fig.33c) leads to a greater pressure difference between two sides of the blade. Note that the pressure distribution pattern is similar to that in Fig.33a, unless the rotation speed is too high (large tip speed ratio, as in Fig.33b) to be practically achieved. 

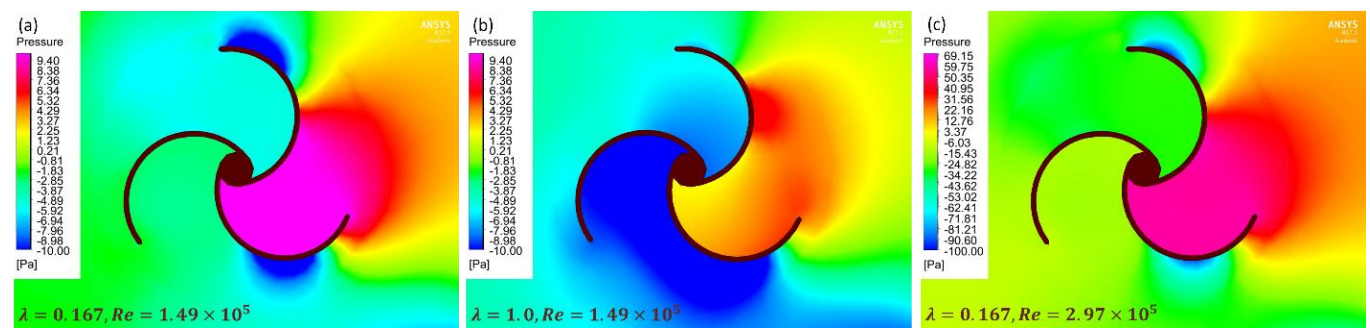

Fig.33Pressure distribution around energy harvester $A$ at (a) $\lambda=0.167, \operatorname{Re}=1.49 \times 10^{5}$, (b) $\lambda=1.0, R e=1.49 \times 10^{5}$ and (c) $\lambda=0.167, R e=2.97 \times 10^{5}$.

Within one revolution, the instantaneous torque is found to be non-uniform as shown in Fig.34. It is also observed in the ranges of a rotor angle that the instantaneous dynamic torque coefficient is negative, the harvester is in an unfavorable position to sustain rotation. It can be seen that although at $\lambda=1.0$ and $\lambda=1.167$ the highest power coefficient can be theoretically achieved at $R e=1.49 \times 10^{5}$ and $R e=2.97 \times 10^{5}$ respectively, due to the negative instantaneous dynamic torque coefficient it is more unlike to be practically realized. At both Reynolds numbers, when the tip speed ratio is less than a certain critical value, which makes the instantaneous dynamic torque coefficients to be all positive throughout the whole revolution. This tip speed ratio corresponds to the rotation speed that the harvester could experimentally achieve, which is $\lambda=0.167$ when $R e=1.49 \times 10^{5}$ and $\lambda=0.5$ when $R e=2.97 \times 10^{5}$. 

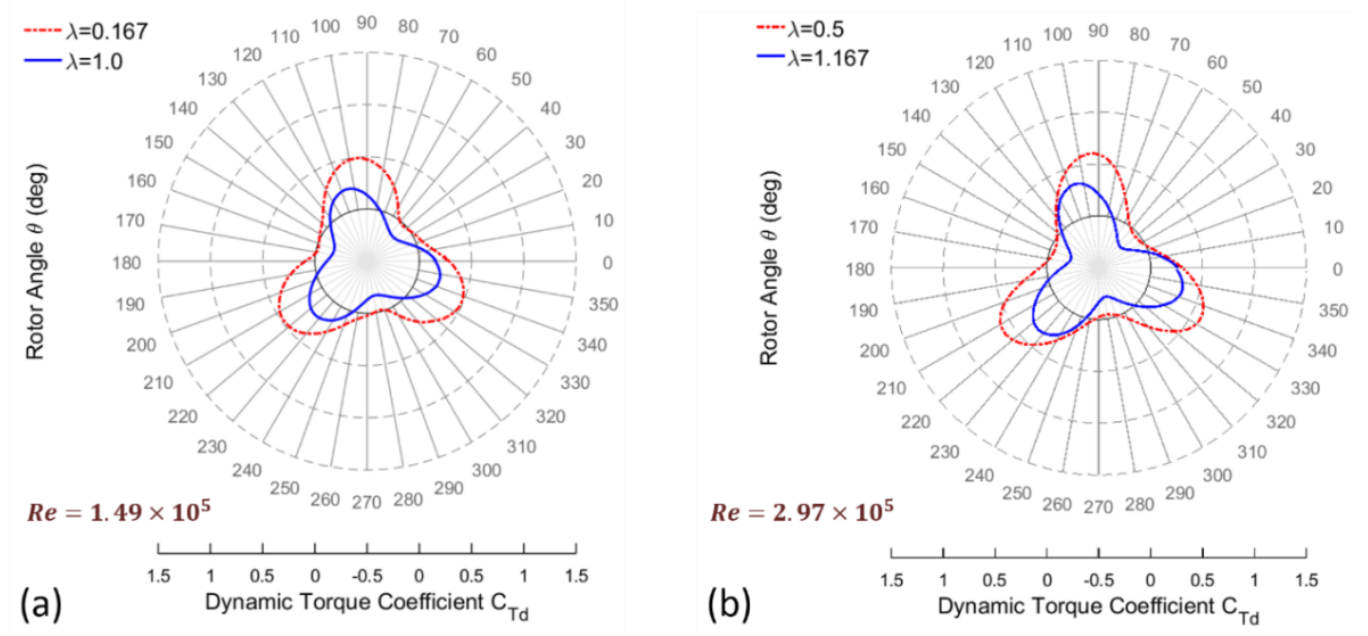

Fig.34 Instantaneous dynamic torque coefficient at (a) $\operatorname{Re}=1.49 \times 10^{5}$ : at $\lambda=1.0$ the highest possible power coefficient is achieved and at $\lambda=0.167$ the rotation speed is set to be the same in experiments. And at (b) $\operatorname{Re}=2.97 \times 10^{5}$ : at $\lambda=1.25$ the highest possible power coefficient is achieved and at $\lambda=0.5$ the rotation speed is set to be the same in experiments.

Dynamic study of harvester A involves a wide range of the tip speed ratio to gain insight on the trend of power coefficient variation. However, high tip speed ratio is more unlikely to be achieved, since flow/mechanical friction limits possible attainable rotation speed, and it is not physical for the harvester rotating faster than the oncoming air flow without any external driving forces. Dynamic studies of other energy harvesters, the tip speed ratios ranges is found to be corresponding to the range that can be possibly practically achieved, which is narrower than the case for harvester A. For this, the rotation speed of the harvester is set to be in the range of 5 to 140 radians per second.

\subsubsection{Effect of the blade number $N$}

Both energy harvesters $\mathrm{B}$ and $\mathrm{C}$ are designed and fabricated to investigate the effect of the blade number $(\mathrm{N})$ of energy harvester. The design details of harvester 
B with two blades $(\mathrm{N}=2)$ and harvester $\mathrm{C}$ with four blades $(\mathrm{N}=4)$ can be found in Appendix A.

Harvester B does not self-start to rotate, even when the Reynolds number is increased to as high as $9.90 \times 10^{5}$. Thus in practice, it needs a 'triggering' force to initiate rotation. Once the harvester B is triggered to rotate, it can sustain rotation and harness energy until the Reynolds number is reduced to $2.80 \times 10^{5}$. As compared to harvester $\mathrm{A}$, harvester $\mathrm{B}$ has higher cut-in Reynolds number and a narrower operational range. The overall energy conversion efficiency is also evaluated. The experimental measurements results can be found in Appendix B. The electrical power output measured and energy conversion efficiency are evaluated in the same way as harvester A. The rotation speed, electrical power generated and energy conversion efficiency varied with Reynolds number is shown in Fig.35. 'Saturation' in rotation speed is also observed for harvester B at high Reynolds numbers, and so does the electrical power output. For the same reason as discussed for harvester A, a drop in overall energy conversion efficiency occurs at high Reynolds numbers. 

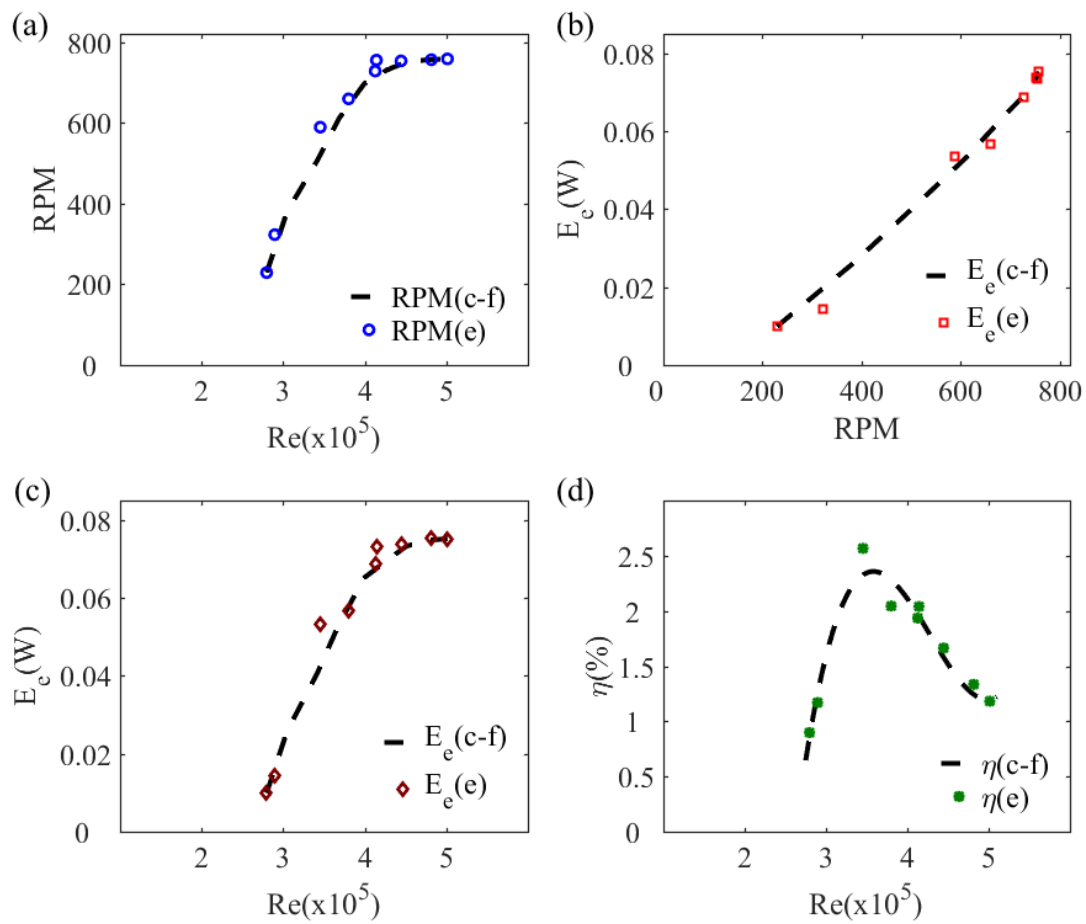

Fig.35 (a) RPM variation with Reynolds number. (b) Electrical power output varied with RPM. (c) Electrical power and (d) Overall energy conversion efficiency varied with Reynolds number for energy harvester B. (c-f denotes curve fitting and e denotes experimental results).

Similar performance evaluation is performed on energy harvester $\mathrm{C}$ with four blades. It is experimentally shown that harvester $\mathrm{C}$ is associated with a cut-in Reynolds number of $8.89 \times 10^{5}$, at which it self-starts rotation without any external triggering force. Once rotation is initiated, harvester $\mathrm{C}$ is able to sustain rotation as long as the Reynolds number is above $3.69 \times 10^{5}$. Same as harvester B, harvester $\mathrm{C}$ possesses higher cut-in Reynolds number and narrower operational range as compared to benchmark harvester A. Experimental results for evaluating overall energy conversion efficiency of harvester $\mathrm{C}$ can be found in Appendix B. Variations of the rotation speed, electrical power output and overall energy conversion efficiency with Reynolds number are shown in Fig.36. Ascending 
trends are observed for rotation speed, electrical power output and energy conversion efficiency with increased Reynolds number. No 'saturation' in rotation speed or electrical power output is observed in the test range of Reynolds numbers. Also, the similar drop in energy conversion efficiency as harvester A and B is not observed for harvester $\mathrm{C}$ in the test range. This is due to that within the Reynolds number test range, the air flow rate is not large enough to drive the energy harvester C to reach its 'saturated' rotation speed. Such 'saturation' is expected to take place at higher Reynolds number, beyond the present test range.
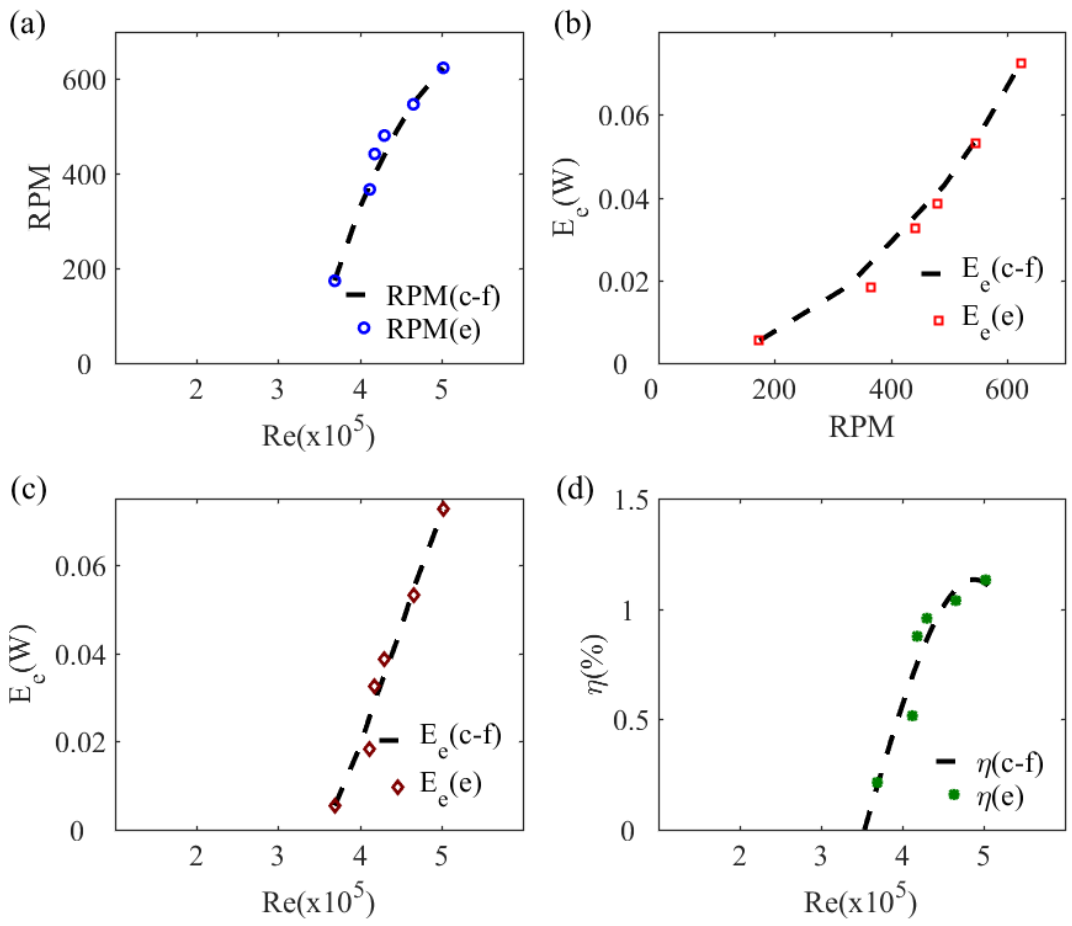

Fig.36 (a) RPM variation with Reynolds number. (b) Electrical power output varied with RPM. (c) Electrical power and (d) Overall energy conversion efficiency varied with Reynolds number for energy harvester $C$. (c-f denotes curve fitting and $e$ denotes experimental results). 
The performances of harvester A, B, and C are compared terms of overall energy conversion efficiency and summarized in Fig.37. It can be seen that three-blade harvester $\mathrm{A}$ is associated with highest maximum overall energy conversion efficiency among three energy harvester models. Additionally, three-blade energy harvester also possesses wider operational range and smaller cut-in Reynolds number, which are more desirable for an energy harvester to be utilized. The smaller energy conversion efficiency of harvester B is possibly due to the open space between blades being too wide and makes it less effective in capturing oncoming air mass to build up pressure difference. The smaller efficiency of harvester $\mathrm{C}$ with four blades is likely due to its heavier weight contributed by its extra blade. Also, one more blade may hinder air flow to reach centre part of harvester. It is reasonable to conclude that an optimum opening and space between blades are needed, so that air mass can flow into the energy harvester and be concentrated between the blades. Air mass concentration is necessary and essential for building up of pressure difference between the sides of blade, and subsequently the pressure difference drives harvester rotation by generating large torque. Therefore, three-blade harvester is an optimum choice, as the opening is wide enough for air mass to enter and the space between the blades is small enough for a sufficiently large pressure difference to be built up. 


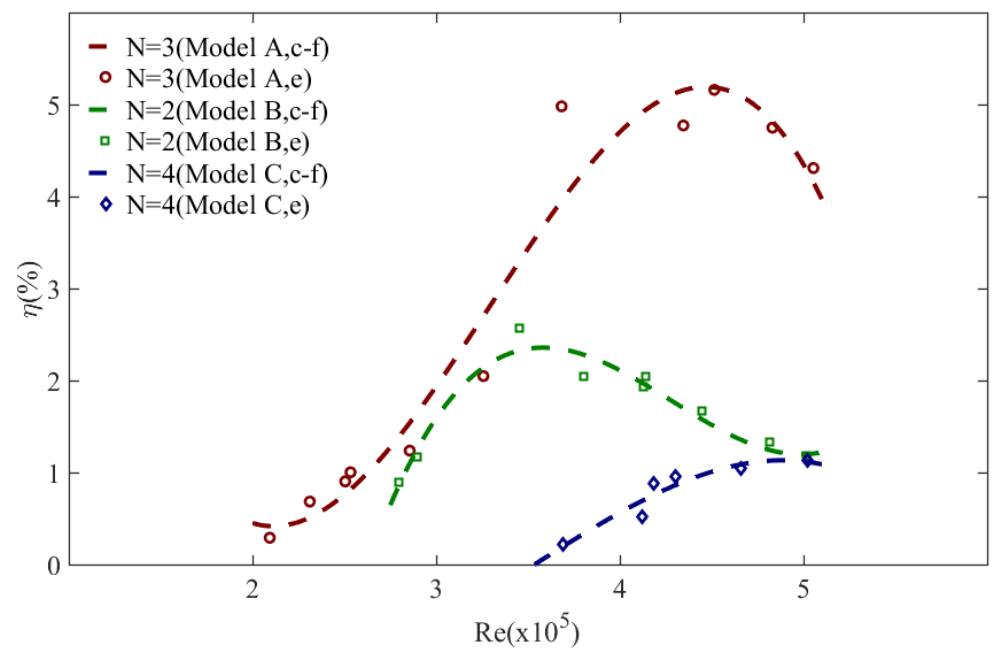

Fig.37 Comparison of overall energy conversion efficiency from harvester A, B and C with different blade numbers, as the Reynolds number is increased. (c-f denotes curve fitting and e denotes experimental results).

Foregoing measurements reveal that the energy harvester with three blades performs better than those with two or four blades. Therefore, in the following parametric measurements, all other energy harvester models, namely harvester $\mathrm{D}$ to $\mathrm{J}$, are designed with three blades.

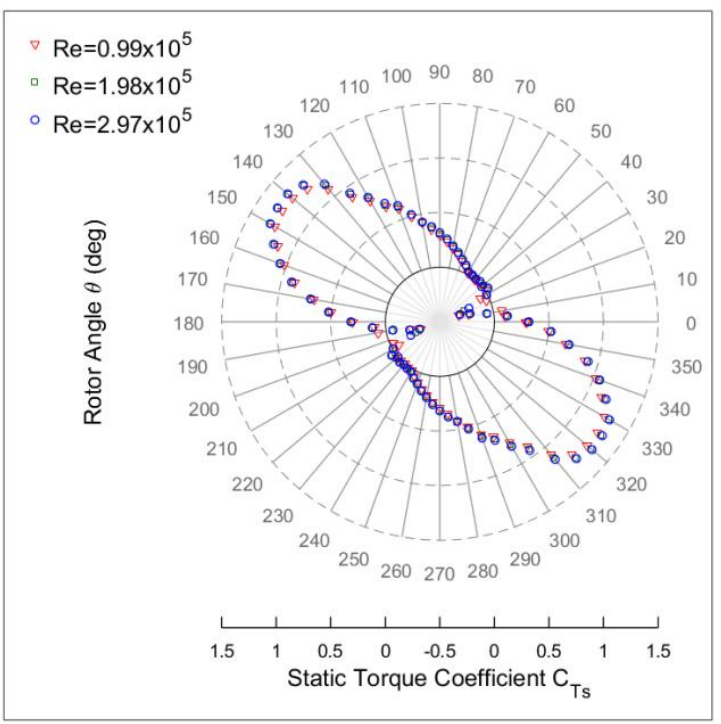


Fig.38 Variation of static torque coefficients at different rotor angles at different Reynolds numbers for harvester $B$.

Numerical studies are also conducted on harvester B and C. Fig.38 is showing the variation of static torque coefficient at different rotor angles for harvester $\mathrm{B}$. Increasing Reynolds number does not significantly increase values of static torque coefficient. Both negative and positive static torque coefficient values exist. The static torque coefficient is positive in the rotor angle ranges of $0^{\circ}$ to $5^{\circ}, 35^{\circ}$ to $185^{\circ}$ and $215^{\circ}$ to $360^{\circ}$, and it means the energy harvester is more likely to self-start rotation at rotor angles within these ranges. The maximum static torque coefficient value take place at rotor angles of $140^{\circ}$ and $320^{\circ}$, while the minimum values are at rotor angle of $20^{\circ}$ and $200^{\circ}$, indicating the favorable and unfavorable positions for self-start rotation, respectively. It is observed that its maximum static torque coefficient value is larger than that of harvester $\mathrm{A}$, and the minimum static torque coefficient value is more negative than that of harvester $\mathrm{A}$ as well.

The variation of static torque coefficient for harvester $\mathrm{C}$ is shown in Fig.39. Positive static torque coefficients exist at rotor angle ranges of $0^{\circ}-20^{\circ}, 45^{\circ}-110^{\circ}$, $135^{\circ}-200^{\circ}, 225^{\circ}-290^{\circ}$ and $315^{\circ}-360^{\circ}$. Maximum static torque coefficient values are observed at $65^{\circ}, 155^{\circ}, 245^{\circ}$ and $335^{\circ}$, while minimum values take place at $30^{\circ}$, $120^{\circ}, 210^{\circ}$ and $300^{\circ}$. As compared to harvester $\mathrm{A}$, it can be seen that the maximum static torque coefficient value is lower, and the minimum static torque coefficient is less negative. 


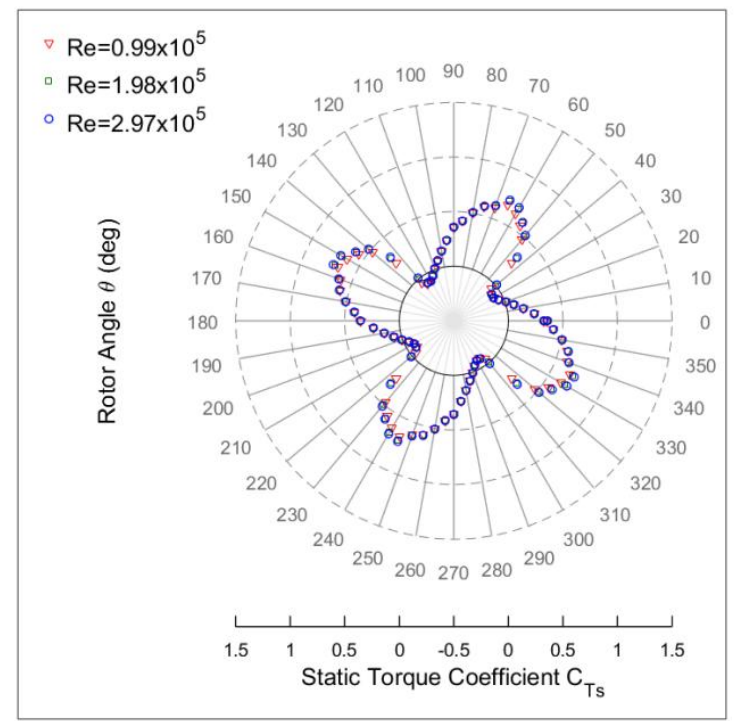

Fig.39 Variation of static torque coefficients at different rotor angles at different Reynolds numbers for harvester $\mathrm{C}$.

Some interesting points are to be made about static performances of energy harvester models with different blades numbers. Firstly, the number of lobes formed in static torque coefficient diagram corresponds to the blade number. This is easily explained by the periodic structure of the energy harvesters. Secondly, increasing blade number seems to reduce variation of static torque coefficient values at different rotor angles and make it more uniform. The maximum static torque coefficient is less positive with increased blade number, and the minimum static torque coefficient is less negative with increased blade number. Further increasing blade number may possibly make the static torque coefficient values more uniform at all rotor angles, which is very desirable for energy harvester to self-start rotation. However, it must be remembered that increasing blade number will severely increase energy harvester weight and made the harvester less likely to self-start rotation. This is the primary reason for four-blade energy harvester to 
possess higher cut-in Reynolds number than three-blade harvester experimentally, even though the numerical static torque coefficients are more uniformly positive.

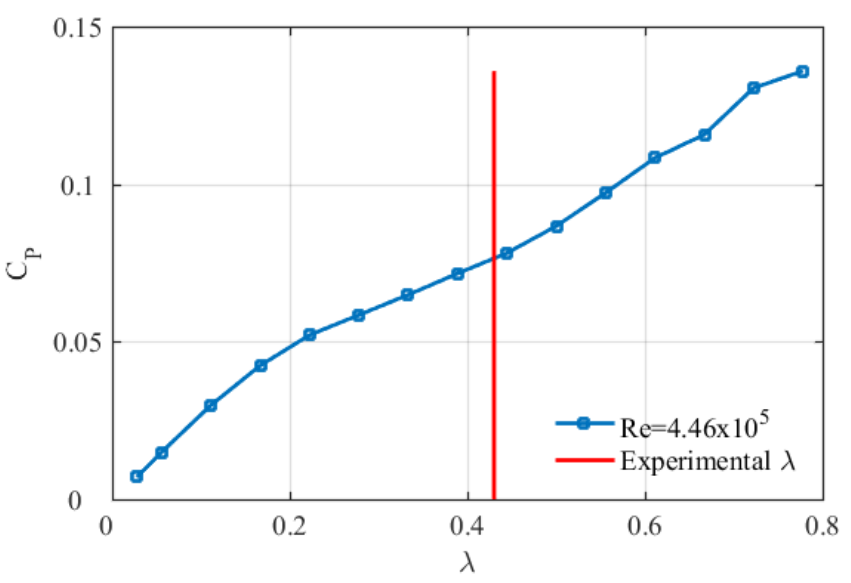

Fig.40 Performance characteristic curves for harvester $B$ at $R e=4.46 \times 10^{5}$. (The blue line indicates values attained by numerical work and the red line indicates experimentally achieved tip speed ratio).

The dynamic performance of energy harvester B is studied by attaining its characteristic curve at Reynolds number of $4.46 \times 10^{5}$ while its rotation speed is set between 5 and 140 radians per second (see Fig.40). In the tip speed ratio range, the characteristic curve is at portion of increasing trend. As compared to harvester A, the power coefficient is lower at the same tip speed ratio. This indicates harvester B is harnessing energy at lower efficiency as compared to harvester $A$. Additionally, the tip speed ratio practically achieved by harvester B is lower than that of harvester $A(\lambda=0.66)$, and makes harvester $B$ functioning at a lower efficiency. The difference between the power coefficient and experimentally attained energy conversion efficiency is due to many factors, and the efficiency of the electromagnetic energy converter will be the most dominant contributor. 


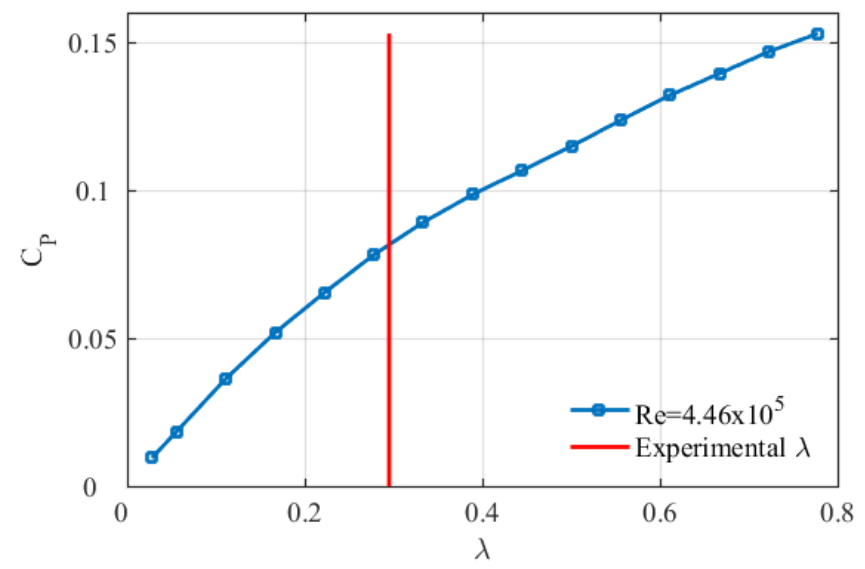

Fig.41 Performance characteristic curves for harvester $C$ at $\operatorname{Re}=4.46 \times 10^{5}$. (The blue line indicates values attained by numerical work and the red line indicates experimentally achieved tip speed ratio).

Characteristic curve of harvester $C$ at Reynolds number of $4.46 \times 10^{5}$ is attained as well, and it is shown in Fig.41. Comparing power coefficient values of harvester A and $C$ at the same tip speed ratio reveals that harvester $C$ has lower value of power coefficient. This is most likely due to the smaller space available between the harvester blades for pressure to be built up, and this leads to smaller torque generated. In practice, four-blade energy harvester $\mathrm{C}$ is much heavier than harvester A, and made experimentally attainable tip speed ratio much lower than that of harvester A. Therefore, four-blade energy harvester model is harnessing energy from moving air mass with lower efficiency than the three-blade one.

\subsubsection{Effect of the energy harvester geometric size (SR)}

Harvester D and E are designed to study the effect of geometric size on the performance of energy harvester model. They both share the same geometric structure and configuration as that of harvester A. However, for harvester D, its 
geometric size is scaled down to $2 / 3$ of harvester $\mathrm{A}(\mathrm{SR}=2 / 3)$, while harvester $\mathrm{E}$ is enlarged to be $4 / 3$ in size of harvester A ( $\mathrm{SR}=4 / 3)$. The detailed design parameters of harvester D and E can be found in Appendix A.
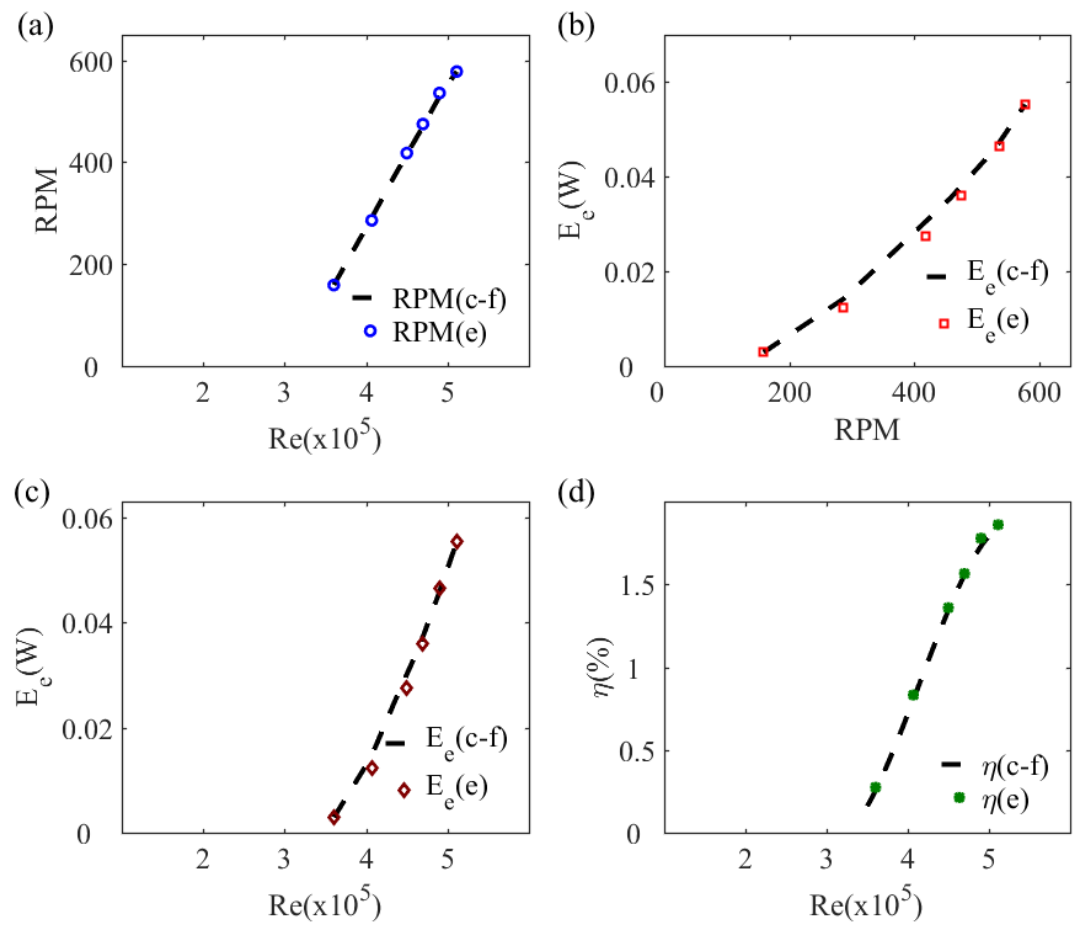

Fig.42 (a) RPM variation with Reynolds number. (b) Electrical power output varied with RPM. (c) Electrical power and (d) Overall energy conversion efficiency varied with Reynolds number for energy harvester $D$. (c-f denotes curve fitting and $e$ denotes experimental results).

Harvester D does not show satisfactory self-starting capability, as it does not selfstart rotation even when the Reynolds number is increased to $9.90 \times 10^{5}$. The operational range of harvester $\mathrm{D}$ is also narrower as compared to harvester $\mathrm{A}$. The minimum Reynolds number at which the energy harvester model can sustain rotation is $3.60 \times 10^{5}$, and this is higher than that of harvester A. Experimental measurement results of induced emf can be found in Appendix B. The electrical 
power output and energy conversion efficiency measured from harvester D are shown inFig.42. It can be seen that the electrical power output and energy conversion efficiency both increase with increased Reynolds number and rotation speed. It is observed that within the test range, the energy conversion efficiency maintains an increasing trend and the maximum energy conversion efficiency is not reached yet. This may indicate that higher conversion efficiency can possibly be achieved at a higher Reynolds number.
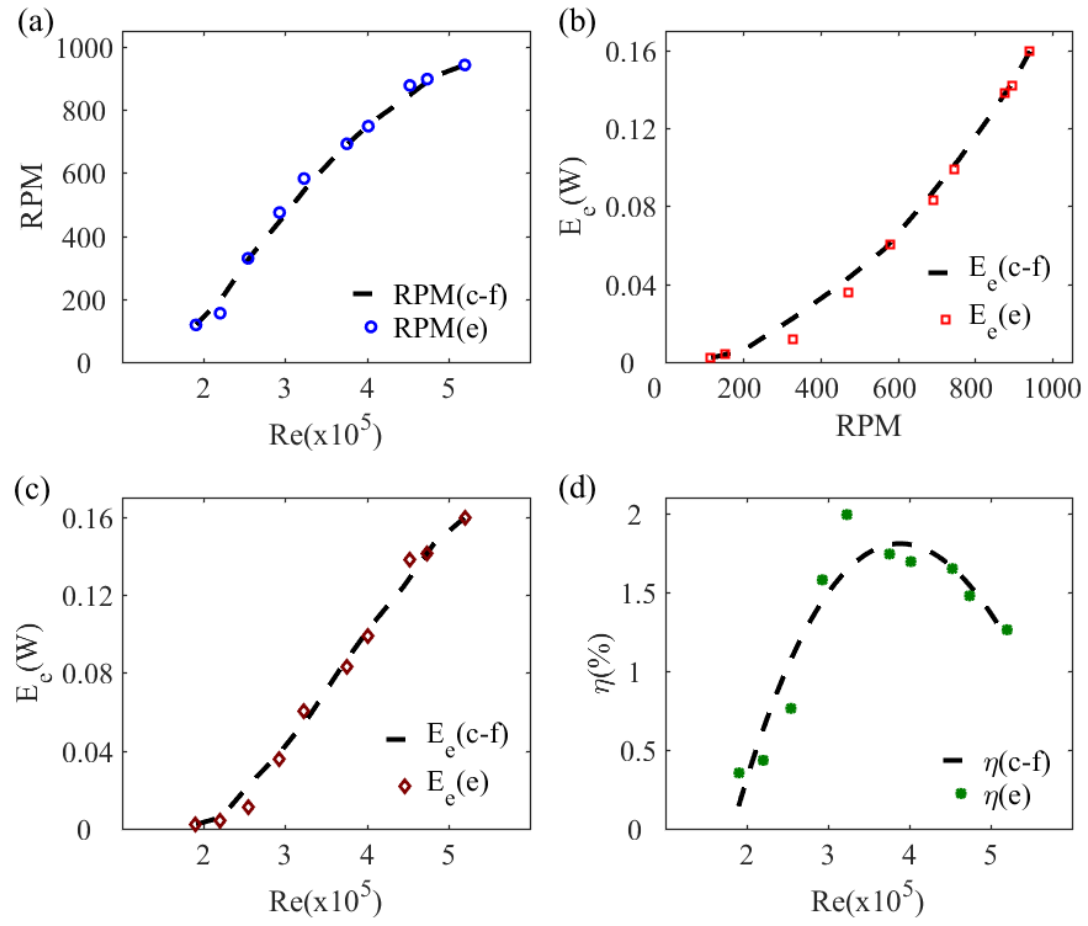

Fig.43 (a) RPM variation with Reynolds number. (b) Electrical power output varied with RPM. (c) Electrical power and (d) Overall energy conversion efficiency varied with Reynolds number for energy harvester $E$. (c-f denotes curve fitting and e denotes experimental results).

The performance of a larger energy harvester with $\mathrm{SR}=4 / 3$, harvester $\mathrm{E}$, is also explored. Experimental measurements of induced emf are provided in Appendix B. 
From Fig.43 it can be seen that rotation speed and electrical power output increase with increased Reynolds number as expected. It is also noticed that the rotation speed gradually 'saturates' at high Reynolds numbers, thus the overall energy conversion efficiency starts to decline when Reynolds number is higher. Additionally, harvester $\mathrm{E}$ shows improvement in operational range, as it can maintain rotation at a lower Reynolds number $\left(1.95 \times 10^{5}\right)$ than harvester $\mathrm{A}$. Unfortunately, the static performance is unsatisfactory, as no self-starting rotation is observed at any Reynolds number below $9.90 \times 10^{5}$.

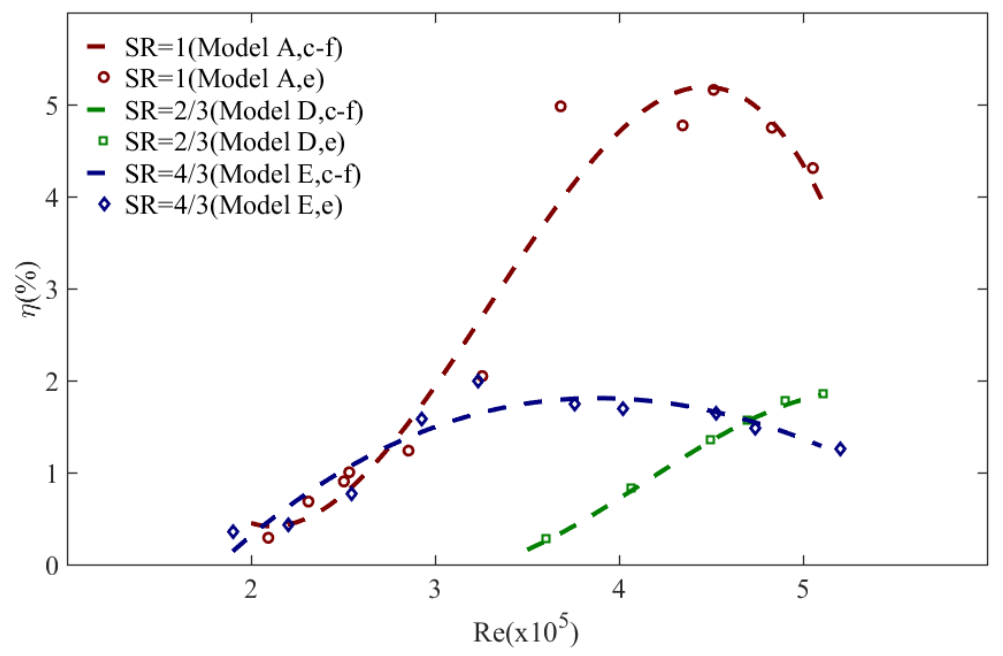

Fig.44 Comparison of overall energy conversion efficiency from harvester A, D and E with different geometric sizes, as the Reynolds number is increased. (c-f denotes curve fitting and e denotes experimental results).

Fig.44 illustrates the comparison of the performances of harvester A, D and E in terms of energy conversion efficiency. The comparison results show harvester A is associated with higher energy conversion efficiency. However, it is to be noted that the smaller energy harvester, harvester D, is still within its raising trend regime. This may possibly mean that harvester D can achieve higher energy conversion 
efficiency at higher Reynolds number, beyond the test range. Another point to be noted is that larger energy harvester model seems to be associated with larger operation range. With increment in energy harvester model size, the minimum Reynolds number that the harvester can remain rotation is lowered, thus a wider operation range is achieved. The reason of larger-size energy harvesters being rotating at lower Reynolds number is that they have longer torque arm, thus generate larger torque to drive harvesters to rotate. However, the enhancement effect of longer torque arm is limited by energy harvester weight, as heavier energy harvesters will be more difficult to be rotating fast.

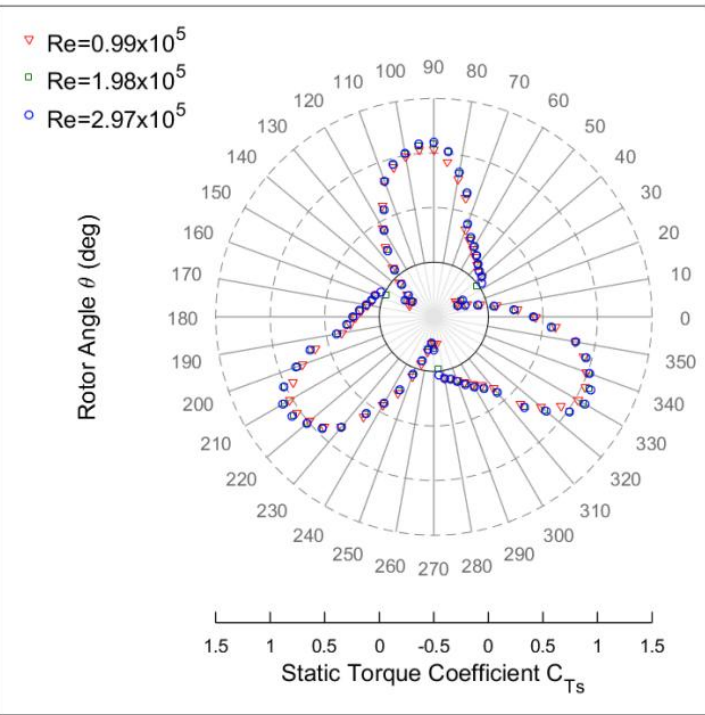

Fig.45 Variation of static torque coefficients at different rotor angles at different Reynolds numbers for harvester $D$.

Static performance of harvester D and E are numerically studied. The variation of static torque coefficient of harvester D is shown in Fig.45. Positive and negative values of static torque coefficient both exist at different rotor angles. Positive static 
torque coefficient values are attained in rotor angle ranges of $0^{\circ}-10^{\circ}, 40^{\circ}-130^{\circ}$, $160^{\circ}-250^{\circ}$, and $280^{\circ}-360^{\circ}$. The maximum static torque coefficient value take place at rotor angles of $90^{\circ}, 210^{\circ}$ and $330^{\circ}$, while the minimum static torque coefficient values are observed at rotor angles of $25^{\circ}, 145^{\circ}$ and $265^{\circ}$. It is observed that the pattern of static torque coefficient variation is very similar to that of harvester A.

The variation of static torque coefficient of harvester $E$ is shown in Fig.46. The pattern of the plot is similar to that of harvester A, but the static torque coefficient values are more positive. In the rotor angle ranges of $0^{\circ}-20^{\circ}, 35^{\circ}-140^{\circ}, 155^{\circ}-260^{\circ}$ and $275^{\circ}-360^{\circ}$, harvester $\mathrm{E}$ possesses positive static torque coefficients. The maximum static torque coefficient occurs at rotor angles of $95^{\circ}, 215^{\circ}$ and $335^{\circ}$, while the minimum static torque coefficients are observed at $25^{\circ}, 145^{\circ}$ and $265^{\circ}$.

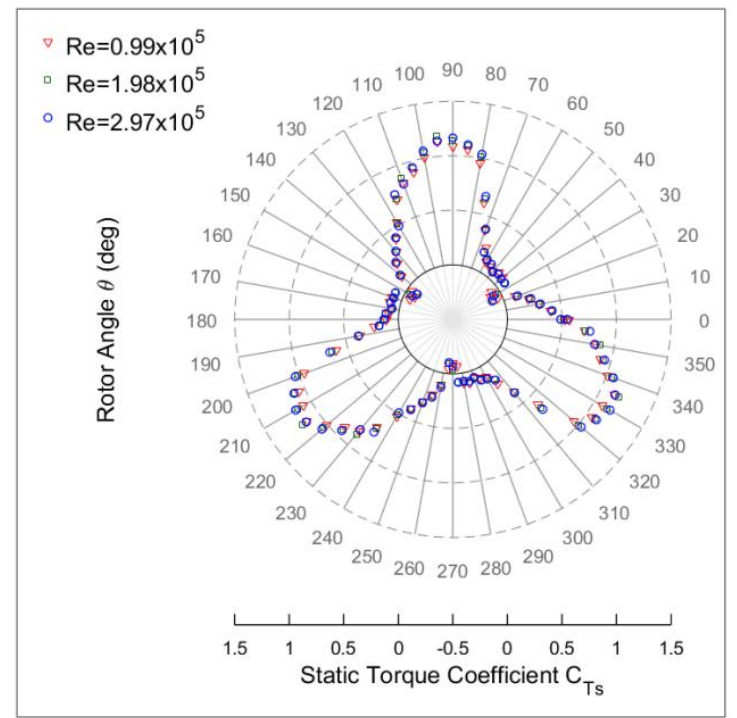

Fig.46 Variation of static torque coefficients at different rotor angles at different Reynolds numbers for harvester $\mathbf{E}$. 
Comparing distributions of static torque coefficient of energy harvester models with different geometric sizes reveal that the same geometric structure will lead to the same static torque coefficient distribution pattern, regardless of difference in geometric size. As the geometric size is increased, the negative ranges of static torque coefficient are narrowed and become less negative. This improvement in static torque coefficient is due to longer torque arm of larger energy harvester.

Numerical study on dynamic performance of harvester D is conducted, and characteristic curve is obtained as shown in Fig.47. The power coefficient of harvester D is comparable to that of harvester $\mathrm{A}$, but due to the smaller torque arm of it, the rotation speed it can experimentally achieve is lower. Therefore, the power coefficient at which the energy harvester functions is lower than that of harvester A.

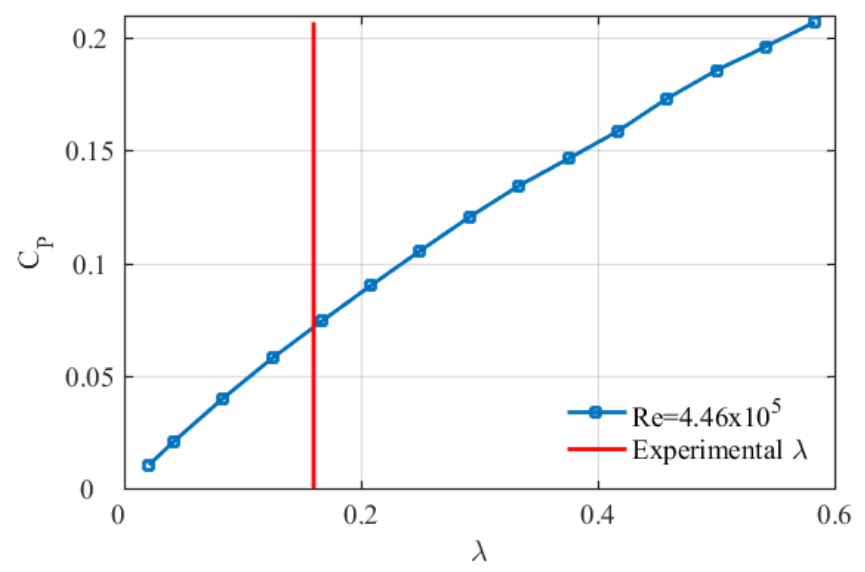

Fig.47 Performance characteristic curves for harvester $D$ at $R e=4.46 \times 10^{5}$. (The blue line indicates values attained by numerical work and the red line indicates experimentally achieved tip speed ratio). 
The power coefficient of harvester $\mathrm{E}$ is slightly lower than harvester $\mathrm{A}$ at a given tip speed ratio, as seen in Fig.48. The experimentally achieved rotation speed of harvester $\mathrm{E}$ is lower than that of harvester $\mathrm{A}$, but as harvester $\mathrm{E}$ is larger in size, they have similar tip speed ratios attained in practice. At the experimental tip speed ratio, the power coefficient of harvester $\mathrm{E}$ is lower than that of harvester $\mathrm{A}$, and this corresponds to lower energy conversion efficiency of it as compared to harvester A.

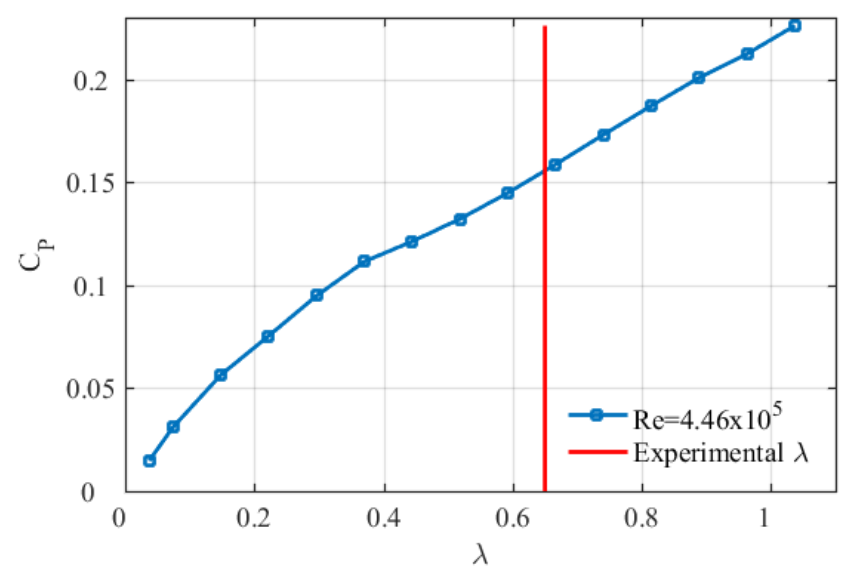

Fig.48 Performance characteristic curves for harvester $E$ at $R e=4.46 \times 10^{5}$. (The blue line indicates values attained by numerical work and the red line indicates experimentally achieved tip speed ratio).

These three energy harvester (A, D and E) have similar geometric structures, but differ in geometric size. It can be seen that for energy harvesters with similar geometric structures, the characteristic curves are also similar in shape and amplitude. This can be explained as for an energy harvester model at a given rotation speed, enlarging its geometric size is equivalent to increasing the tip speed ratio. Therefore, it can be concluded that the geometric structure is the dominant factor that determined the characteristic curve of the energy harvester model, and geometric size has less effect on the shape of characteristic curve. However, 
geometric size has effect on the tip speed ratio that can be practically attained, thus affects the dynamic performance of energy harvester.

\subsubsection{Effect of the energy harvester aspect ratio $(A R)$}

(a)

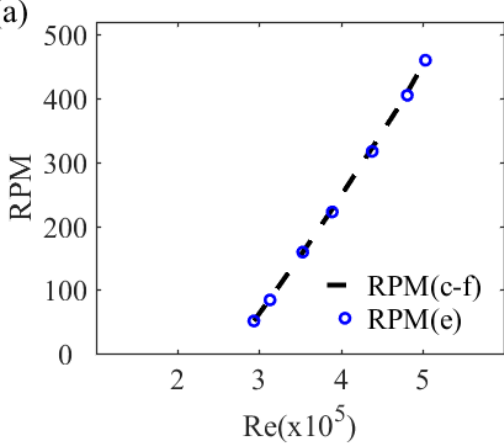

(c)

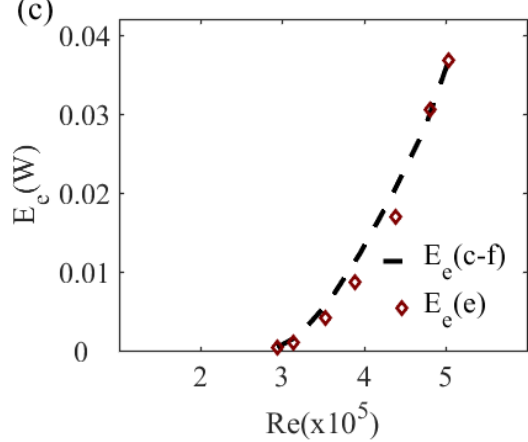

(b)

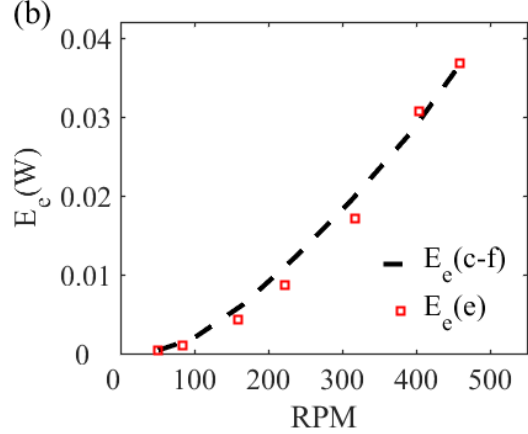

(d)

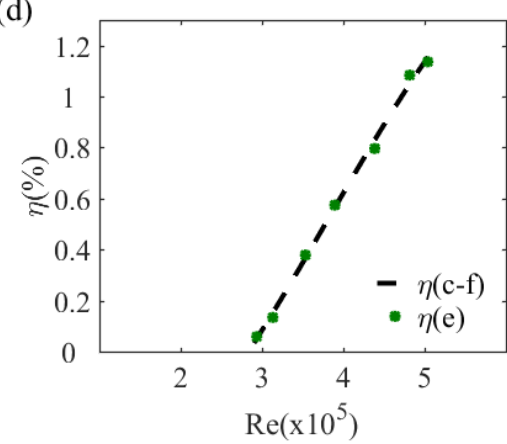

Fig.49 (a) RPM variation with Reynolds number. (b) Electrical power output varied with RPM. (c) Electrical power and (d) Overall energy conversion efficiency varied with Reynolds number for energy harvester $F$. (c-f denotes curve fitting and e denotes experimental results).

Now the study continues to investigate the effect of energy harvester aspect ratio, which is defined as the ratio of turbine length to diameter of end plates. Two energy harvester models, $F$ and $G$ are designed and fabricated for this study. Harvester $\mathrm{F}$ has $\mathrm{AR}=1 / 2$, and its harvester length is halved as compared to harvester A while the end plate diameter is kept unchanged. In contrast, harvester $\mathrm{G}$ has $\mathrm{AR}=2$, and its harvester diameter is reduced to half and the harvester length 
is kept unchanged. The details of design parameters of these two energy harvester models can be found in Appendix A.

Harvester F is associated with a cut-in Reynolds number of $7.52 \times 10^{5}$, and this proves its self-starting capability within the test range. It is to be noted that harvester F's cut-in Reynolds number is higher than that of harvester A, and this indicates harvester $\mathrm{F}$ performs worse than harvester $\mathrm{A}$ in terms of self-starting capability. Harvester $\mathrm{F}$ has a narrower range of operational Reynolds number, as indicated by the minimum functional Reynolds number of $2.94 \times 10^{5}$. To explore the energy conversion efficiency of harvester F, induced emf values are measured, and results are provided in Appendix B. Fig.49 provides information about variations in rotation speed and electrical power output with changes in Reynolds number. Increasing trends are observed in rotation speed, electrical power output, and overall energy conversion efficiency, as Reynolds number is increased. As reduction in overall energy conversion efficiency is not observed in the test range yet, and this may indicate a higher efficiency can possibly be achieved at higher Reynolds numbers. 

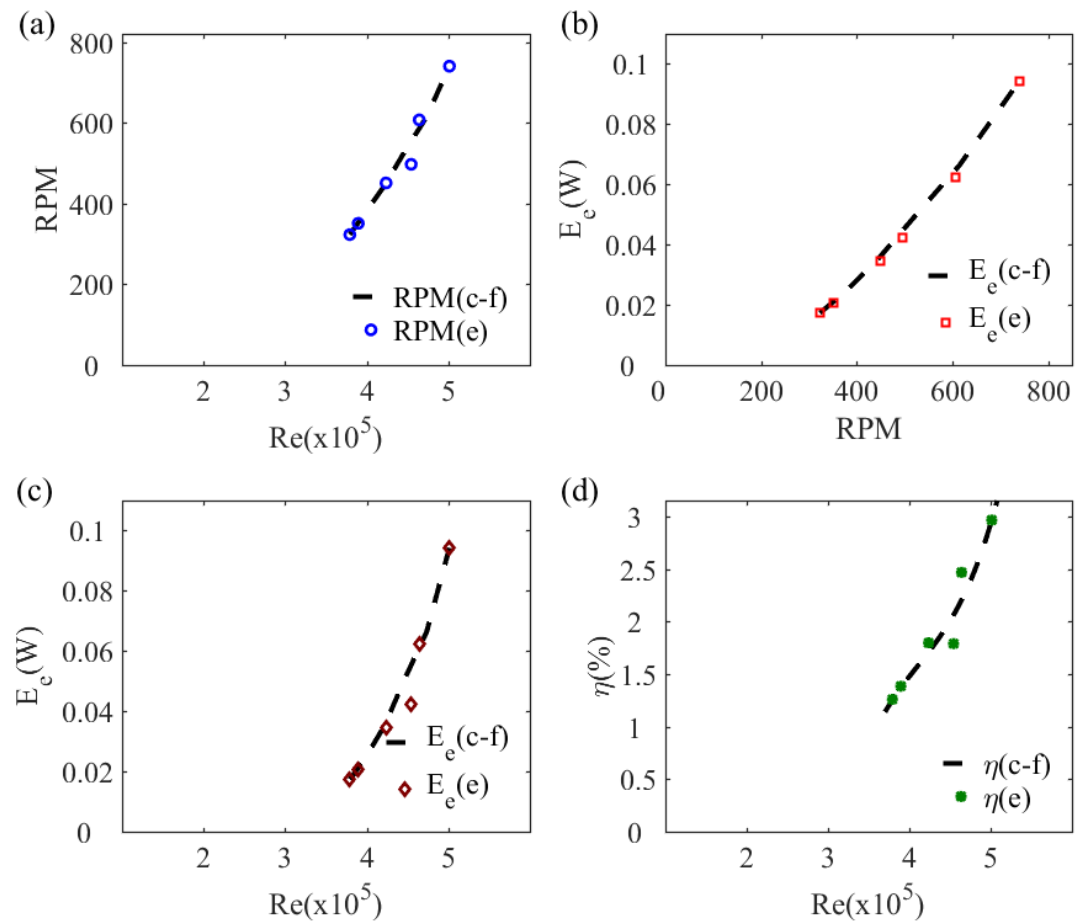

Fig.50 (a) RPM variation with Reynolds number. (b) Electrical power output varied with RPM. (c) Electrical power and (d) Overall energy conversion efficiency varied with Reynolds number for energy harvester G. (c-f denotes curve fitting and e denotes experimental results).

Harvester $\mathrm{G}$ is worse in both self-starting performance and operational Reynolds number range. It is not shown to self-start rotation below Reynolds number of $9.90 \times 10^{5}$. The minimum Reynolds number that the energy harvester model remains rotation was $3.74 \times 10^{5}$. In this two aspects, harvester $\mathrm{G}$ is worse than harvester A. Based on Fig.50, increasing trends of rotation speed, electrical power output and energy conversion efficiency are observed as the Reynolds number is increased. Since the maximum energy conversion efficiency is not reached in the tested Reynolds number range yet, there is possible higher conversion efficiency at higher Reynolds number. 


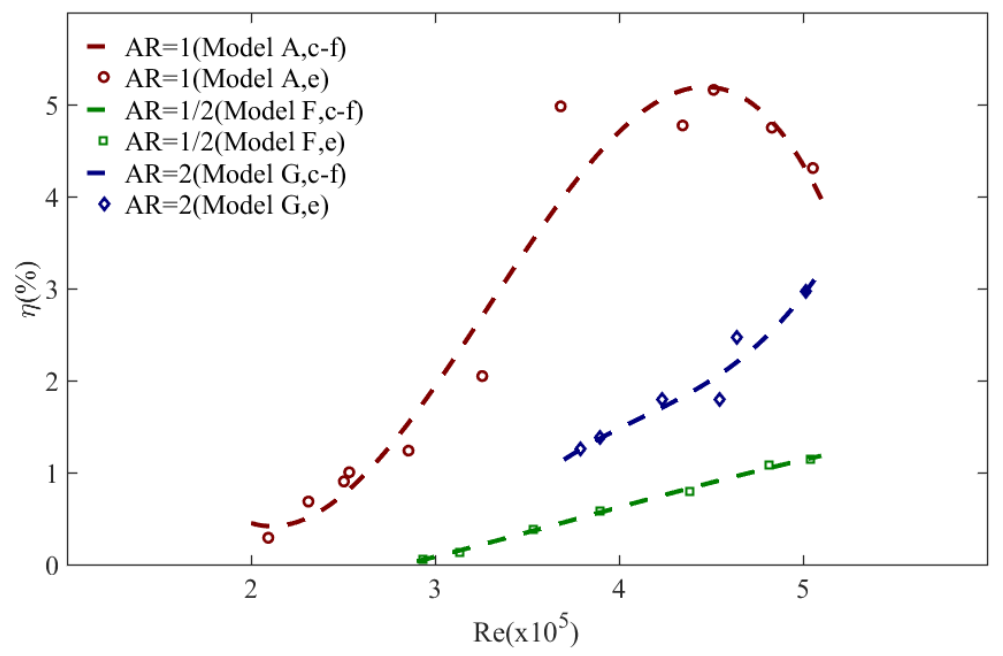

Fig.51 Comparison of overall energy conversion efficiency from harvester A, F and G with different aspect ratios, as the Reynolds number is increased. (c-f denotes curve fitting and e denotes experimental results).

The performances of energy harvester models with different aspect ratios are compared by comparing their energy conversion efficiencies, as shown in Fig.51. It can be seen that harvester $A$ with $A R=1$ is associated with higher energy conversion efficiency, as compared to energy harvester models with higher or lower aspect ratio. For energy harvester with smaller or larger aspect ratio, higher energy conversion efficiency seems to exist at higher Reynolds number. It can be concluded that within test range of Reynolds number, aspect ratio of 1 is the optimum choice. Additionally, it can be seen that harvester A possesses a wider range of operational Reynolds number, and this is desirable for its application. The effect of aspect ratio can be understood as the combined effects of harvester length and space between blades. Energy harvester with aspect ratio of 1/2 or 2 has halved space between blades as compared to harvester A, and this may lead to lower energy conversion efficiency of them. Given the same amount of space between 
blades, higher-aspect-ratio energy harvester performs better in terms of energy conversion efficiency, and this is probably due to wider opening available for oncoming air mass flow to enter.

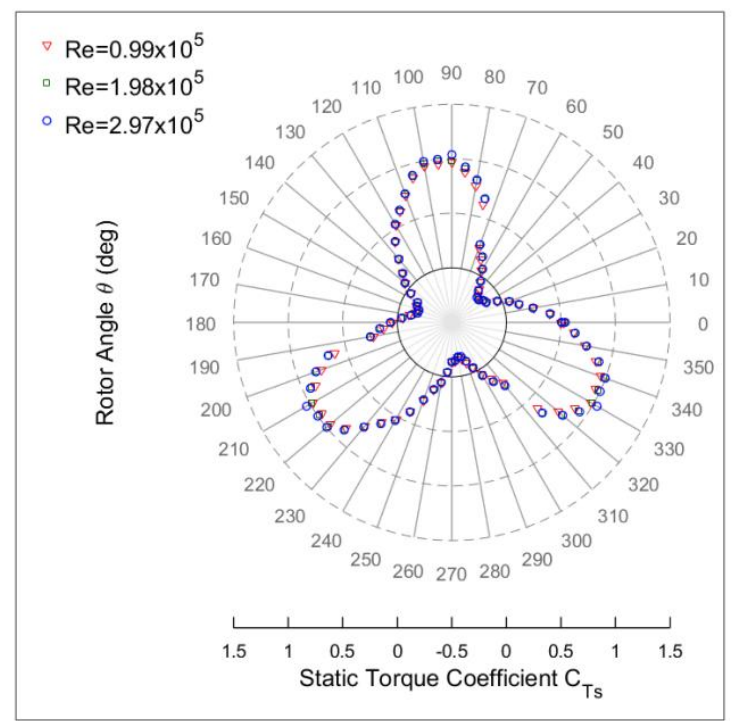

Fig.52 Variation of static torque coefficients at different rotor angles at different Reynolds numbers for harvester $\mathbf{F}$.

Numerical study on static performance of harvester F gives the distribution of static torque coefficient as shown in Fig.52. The geometric structure of harvester F is similar to that of harvester $\mathrm{A}$, thus the pattern of static torque coefficient distribution is also similar to that of harvester A. Both positive and negative values of static torque coefficient exist. In the rotor angle ranges of $0^{\circ}-20^{\circ}, 60^{\circ}-140^{\circ}$, $180^{\circ}-260^{\circ}$ and $300^{\circ}-360^{\circ}$, the static torque coefficient is positive. The maximum static torque coefficient takes place at $95^{\circ}, 215^{\circ}$ and $335^{\circ}$, while the minimum static torque coefficients are at $25^{\circ}, 145^{\circ}$ and $265^{\circ}$. 
The static torque coefficient distribution attained for energy harvester $G$ is shown in Fig.53. Similar distribution pattern is observed for harvester $G$ to that of harvester A. Positive static torque coefficients can be found in the rotor angle ranges of $0^{\circ}-10^{\circ}, 35^{\circ}-130^{\circ}, 155^{\circ}-250^{\circ}$ and $275^{\circ}-360^{\circ}$. Maximum values of static torque coefficient are found at rotor angle of $90^{\circ}, 210^{\circ}$ and $330^{\circ}$, while the minimum values of it are found at $40^{\circ}, 160^{\circ}$ and $280^{\circ}$.

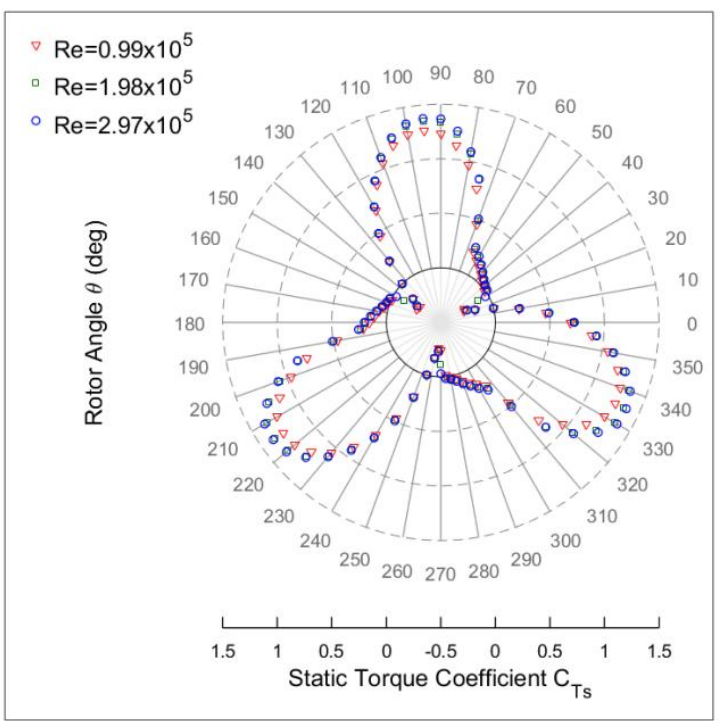

Fig.53 Variation of static torque coefficients at different rotor angles at different Reynolds numbers for harvester $\mathbf{G}$.

Comparing static torque coefficient distributions of energy harvester models with different aspect ratios show that harvesters with similar geometric structures have similar patterns of static torque coefficient distribution. The harvester with larger aspect ratio is associated with ranges of more positive static torque coefficient. This is probably due to the same reason as previously discussed, given the same total space between the blades, higher-aspect-ratio harvester is more efficient in 
capturing air due to larger opening for air to enter. This is also due to the fact if end plates are close to each other, the distorted flows are less effectively concentrated into the harvester.

Dynamic performance of harvester $\mathrm{F}$ is studied as well, and the characteristic curve is obtained as shown in Fig.54. At a given tip speed ratio, the power coefficient of harvester $\mathrm{F}$ is higher than that of harvester $\mathrm{A}$. However, as the space available between the harvester blades is smaller, the energy harvester is less effective in capturing air mass. This leads to smaller rotation speed and tip speed ratio can be attained by the harvester. Consequently, the power coefficient that the energy harvester can practically achieve is much lower than that of harvester A.

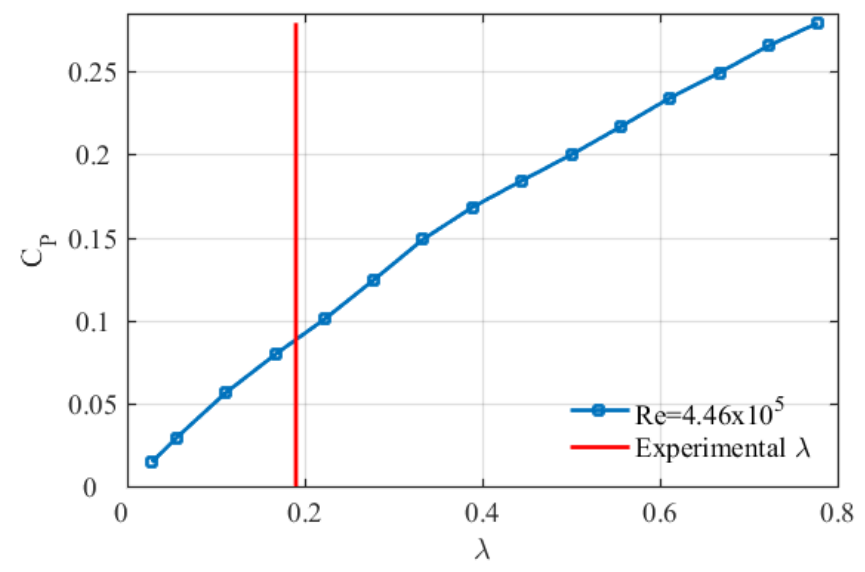

Fig.54 Performance characteristic curves for harvester $F$ at $R e=4.46 \times 10^{5}$. (The blue line indicates values attained by numerical work and the red line indicates experimentally achieved tip speed ratio).

What happened to harvester $\mathrm{G}$ is very similar to that of harvester $\mathrm{F}$, the numerical dynamic study results show that the characteristic curve of harvester $G$ is higher than that of harvester A (Fig.55). This means at a given tip speed ratio, harvester G 
has higher power coefficient that that of harvester A, which is contributed by high dynamic torque coefficient of harvester G. However, the harvester blade of harvester $G$ is much smaller than that of harvester $A$ and less air mass can be captured in a smaller area. This leads to smaller rotation speed that can be achieved, so does the tip speed ratio. As we can see from the characteristic curve of harvester $\mathrm{G}$, at small tip speed ratio, the power coefficient attainable is much lower than that of harvester A. This conclusion corresponds to smaller experimentally observed overall energy conversion efficiency of harvester $G$ as compared to that of harvester A.

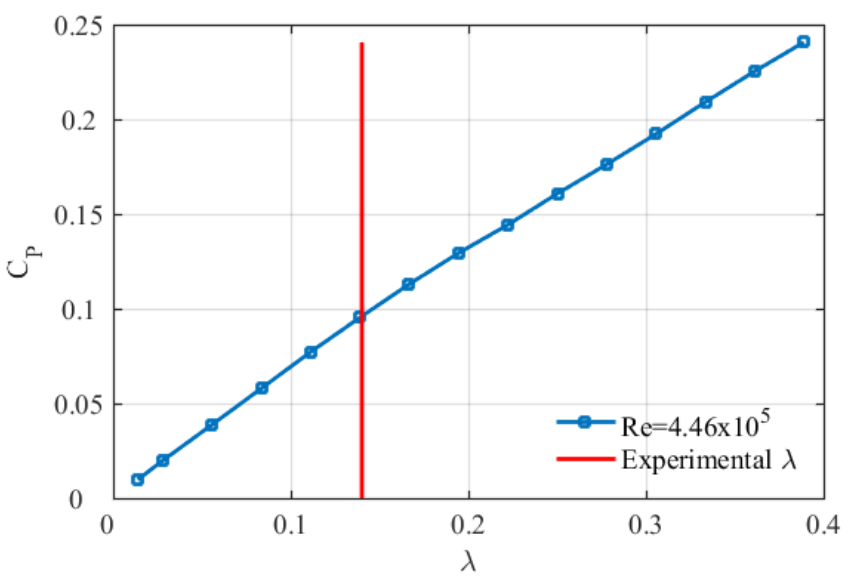

Fig.55 Performance characteristic curves for harvester $\mathrm{G}$ at $\mathrm{Re}=4.46 \times 10^{5}$. (The blue line indicates values attained by numerical work and the red line indicates experimentally achieved tip speed ratio).

\subsubsection{Effect of types of the energy harvester central part}

The effect of types of energy harvester central part is also studied. Harvester $\mathrm{H}$ is designed for this purpose, and it has the solid central shaft removed. As compared 
to harvester A, all the geometric dimensions are the same, but the central part is left as empty gap. The details about harvester design can be found in Appendix A.

Harvester $\mathrm{H}$ is associated with a much lower cut-in Reynolds number as compared to that of harvester $\mathrm{A}$, which is $4.33 \times 10^{5}$. The range of operation Reynolds number is not widened, harvester H's minimum working Reynolds number is $2.70 \times 10^{5}$. Detailed experimental measurements results are provided in Appendix B. The variations in rotation speed, electrical power output and overall energy conversion efficiency are shown in Fig.56. The electrical power output increases with increased rotation speed as expected. Additionally, an obvious 'saturation' in rotation speed is observed, and this leads to reduction in overall energy conversion efficiency at higher Reynolds numbers for the same reason discussed previously.
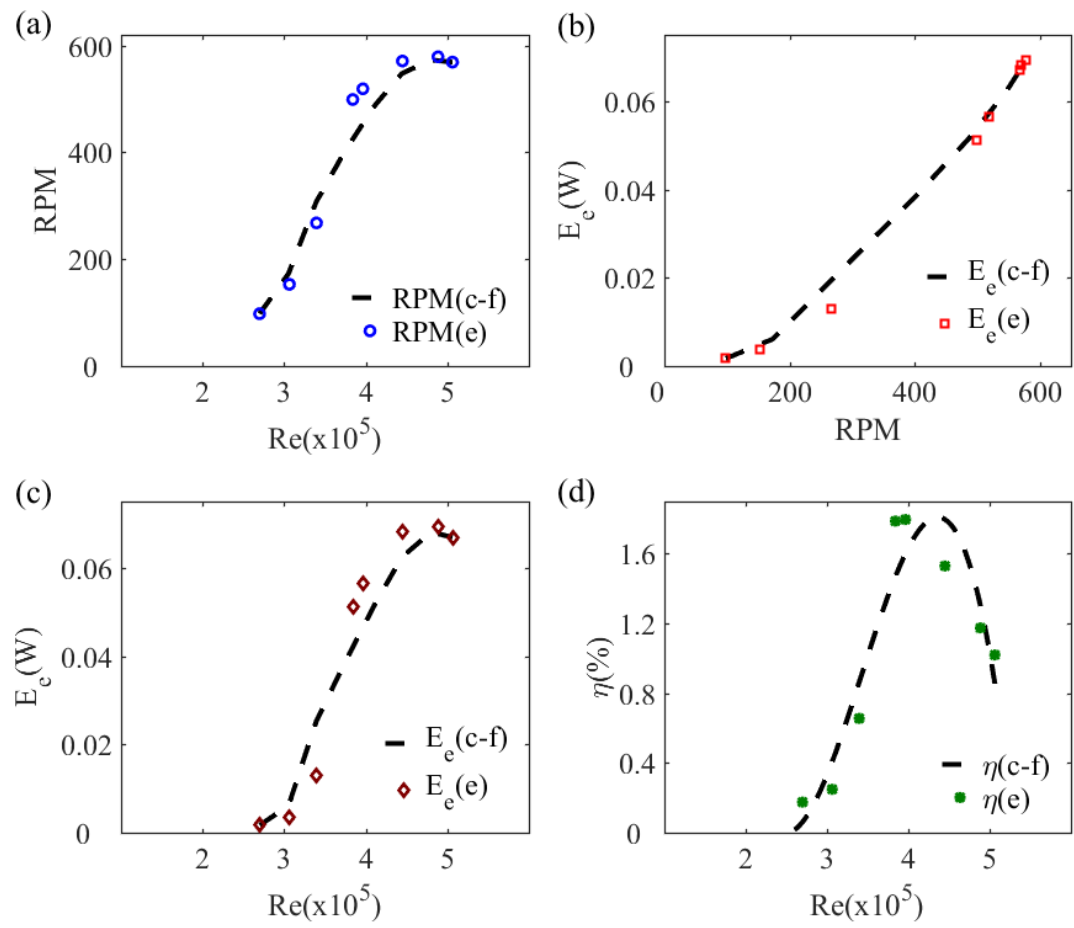
Fig.56 (a) RPM variation with Reynolds number. (b) Electrical power output varied with RPM. (c) Electrical power and (d) Overall energy conversion efficiency varied with Reynolds number for energy harvester $H$. (c-f denotes curve fitting and $e$ denotes experimental results).

Comparison of energy harvesters with and without solid central shaft in terms of overall energy conversion efficiency can be seen in Fig.57. It can be seen that energy harvester with solid central shaft converts wind energy to electrical power more efficiently than the energy harvester with empty central gap. This is due to the fact that removal of central shaft introduces additional pathway for air to pass through, and this significantly reduces the pressure difference between sides of blades. Therefore, the driving force for energy harvester's rotation is also weakened. It has to be noted that the energy harvester with empty central gap has its starting performance enhanced by the same mechanism. When the energy harvester is in static state, the high pressure region at convex side of blade produces torque in counter-rotation direction. With additional air pathway at harvester center, the pressure difference is lowered, and so does the counterrotation torque. Hence energy harvester $\mathrm{H}$ is able to self-start rotation at a lower Reynolds number, as compared to harvester A. 


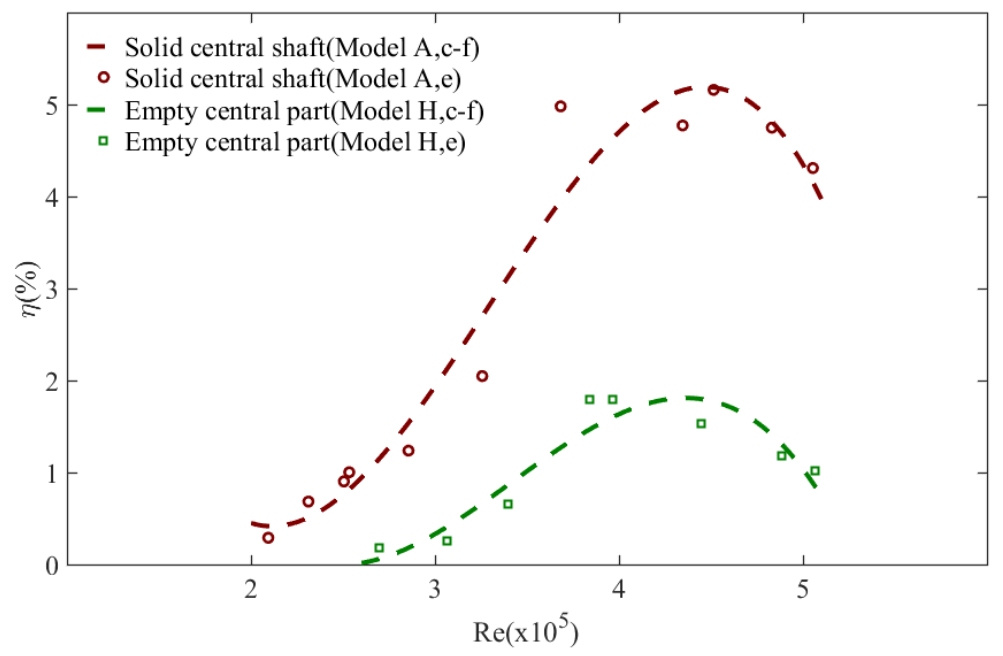

Fig.57 Comparison of overall energy conversion efficiency from harvester $\mathrm{A}$ and $\mathrm{H}$ with different types of central part, as the Reynolds number is increased. (c-f denotes curve fitting and e denotes experimental results).

The static performance of harvester $\mathrm{H}$ is also numerically studied, and finding can be seen in Fig.58. Although periodic pattern still exists in harvester H's static torque coefficient distribution, the pattern is significantly different from that of harvester A. The maximum values of static torque coefficient are observed at rotor angles of $90^{\circ}, 210^{\circ}$ and $330^{\circ}$, while the minimum values of it occur at rotor angles of $55^{\circ}, 175^{\circ}$ and $295^{\circ}$. Throughout the whole range of rotor angles, the static torque coefficient is positive at any rotor angle. This is very desirable for the energy harvester to self-start rotation, and this is consistent with experimentally observed low cut-in Reynolds number. The reason for this is as analyzed previously, the additional air pathway created by removal of solid central shaft reduces the pressure difference between sides of blades, and thus reduces the torque in counter-rotation direction. 


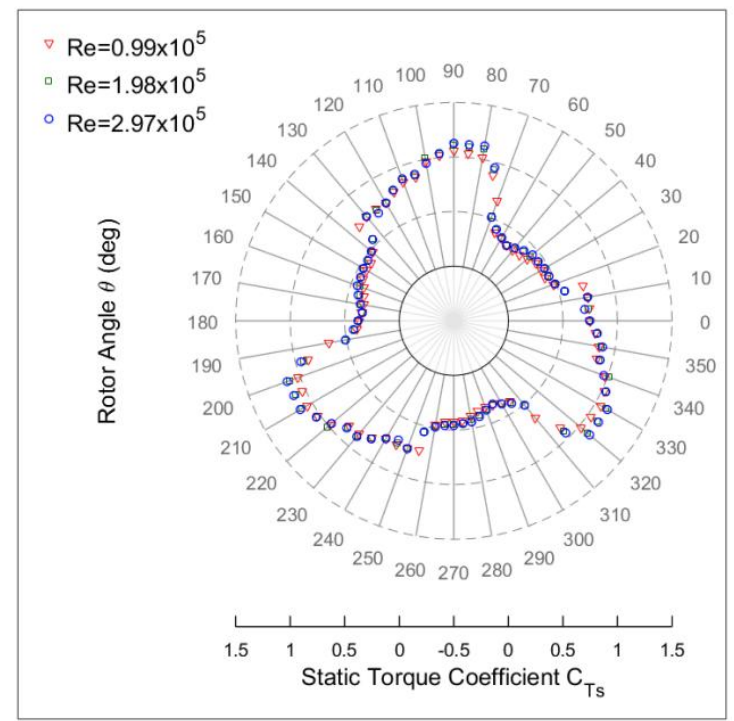

Fig.58 Variation of static torque coefficients at different rotor angles at different Reynolds numbers for harvester $H$.

The reason for improving static performance by removing solid central shaft is explored in details by examining the pressure distribution pattern of harvester $\mathrm{H}$, as seen in Fig.59. Great improvement in static performance is achieved by increasing the minimum static torque coefficient values to positive. This is achieved by reducing the high pressure at convex side of advancing blade by directing air mass to concave side of returning blade via the empty central part. The pressure difference is reduced, so the torque in counter-rotation direction is also reduced. Therefore the experimental cut-in Reynolds number for this harvester $\mathrm{H}$ is much lower. 


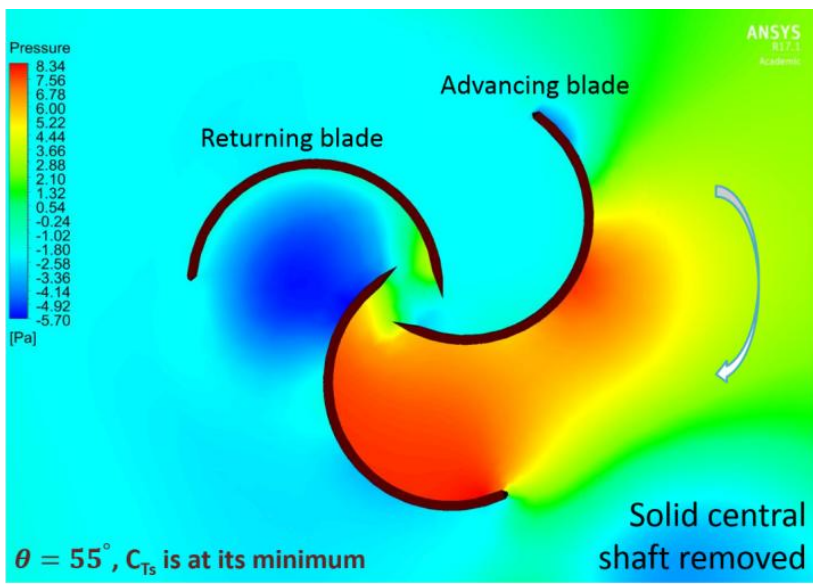

Fig.59 Pressure distribution at minimum static torque coefficient value for energy harvester $\mathbf{H}$.

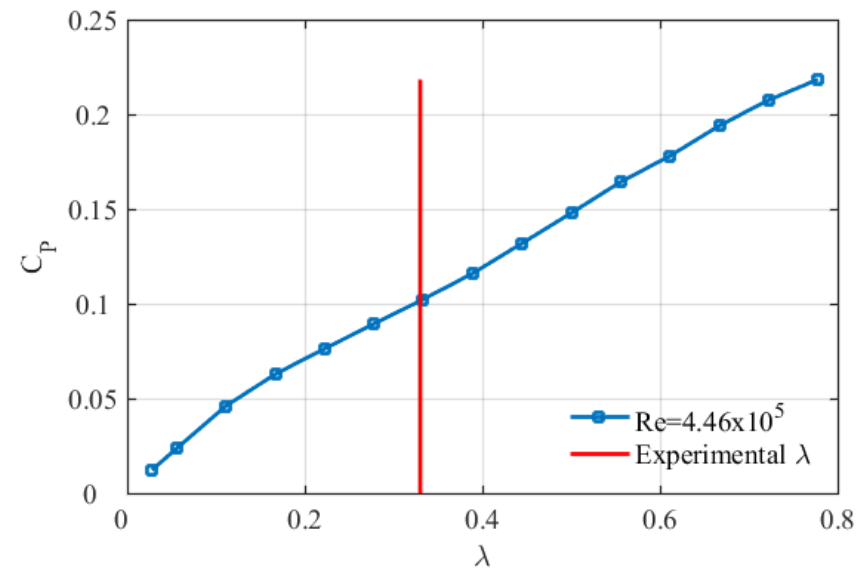

Fig.60 Performance characteristic curves for harvester $H$ at $R e=4.46 \times 10^{5}$. (The blue line indicates values attained by numerical work and the red line indicates experimentally achieved tip speed ratio).

Dynamic performance of harvester $\mathrm{H}$ is numerically studied, and characteristic curve is attained as shown in Fig.60. The shape of harvester H's characteristic curve is similar to that of harvester A, and this is most likely due to their similar geometric structure. However, removal of the solid central shaft has a significant effect on attainable rotation speed and tip speed ratio. Removal of solid central shaft provides a pathway for air trapped to 'escape' and the energy harvester is 
driven to rotate at smaller rotation speed. As compared to harvester A, much lower tip speed ratio leads to a smaller power coefficient of harvester $H$. This is consistent with the lower experimentally observed overall energy conversion efficiency of harvester $\mathrm{H}$.

\subsubsection{Effect of energy harvester end plates}

To investigate the effect of end plates on the performance of energy harvester, harvester I is designed. Harvester I has everything the same as harvester A, but its end plates at both ends are removed. The design details can be found in Appendix A.
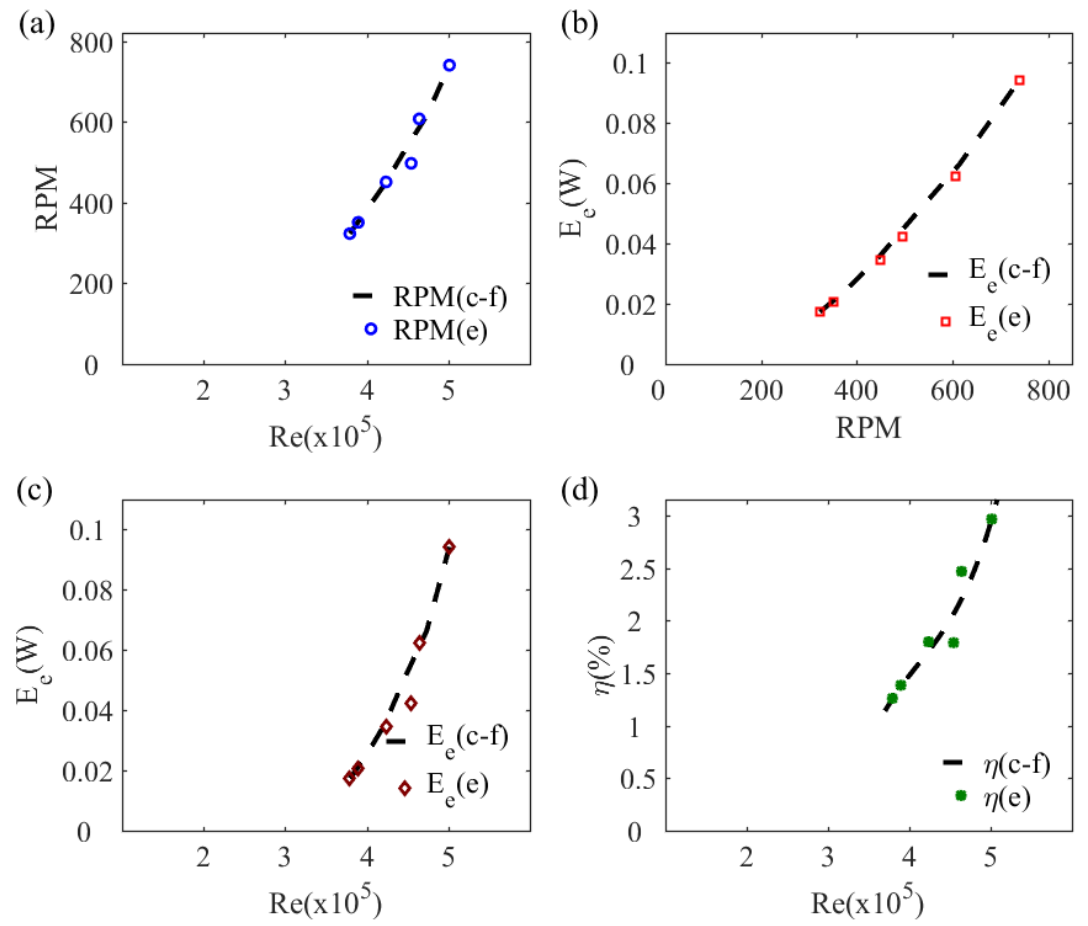

Fig.61 (a) RPM variation with Reynolds number. (b) Electrical power output varied with RPM. (c) Electrical power and (d) Overall energy conversion efficiency varied 
with Reynolds number for energy harvester I. (c-f denotes curve fitting and e denotes experimental results).

Removal of end plates greatly improves the self-starting performance, as the cut-in Reynolds number is lowered to $4.35 \times 10^{5}$. The operational Reynolds number range is smaller, and the minimum Reynolds number that keeps the energy harvester rotating is $3.00 \times 10^{5}$. Detailed induced emf measurement results can be found in Appendix B. The variations of rotation speed, electrical power output and overall energy conversion efficiency are shown in Fig.61. Rotation speed, electrical power output and overall energy conversion efficiency all show increasing trends as Reynolds number is increased. The reduction in overall energy conversion efficiency is not observed in the test range, and this suggests that there probably be a higher overall energy conversion efficiency at higher Reynolds number.

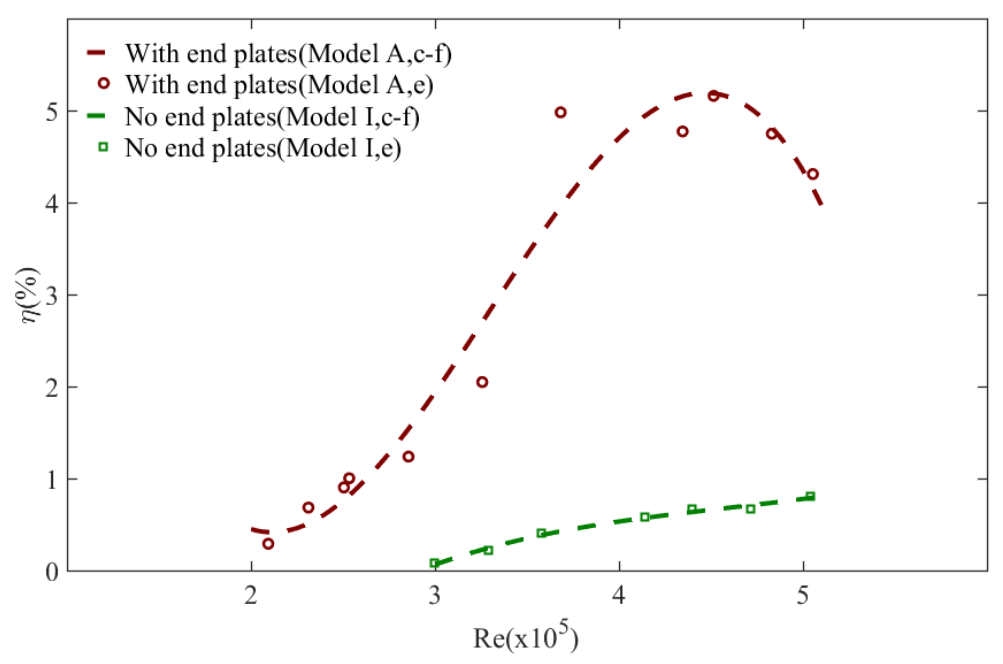

Fig.62 Comparison of overall energy conversion efficiency from harvester $A$ and I with or without end plates, as the Reynolds number is increased. (c-f denotes curve fitting and e denotes experimental results). 
The overall energy conversion efficiencies of energy harvesters with and without end plates are compared in Fig.62. It can be seen that once the end plates are removed, the energy harvester converts to electricity much less efficiently. This low overall energy conversion efficiency is due to the same reason as the case of removing central solid shaft. Removal of end plates creates pathways at the sides for air to flow, and this leads to reduced pressure difference between two sides of harvester blades. Smaller pressure difference provides smaller driving force for harvester to rotate, and therefore caused smaller energy conversion efficiency. For the same mechanism as discussed for the case of harvester with empty central gap, removing end plates from energy harvester model also significantly improves its self-starting capability.

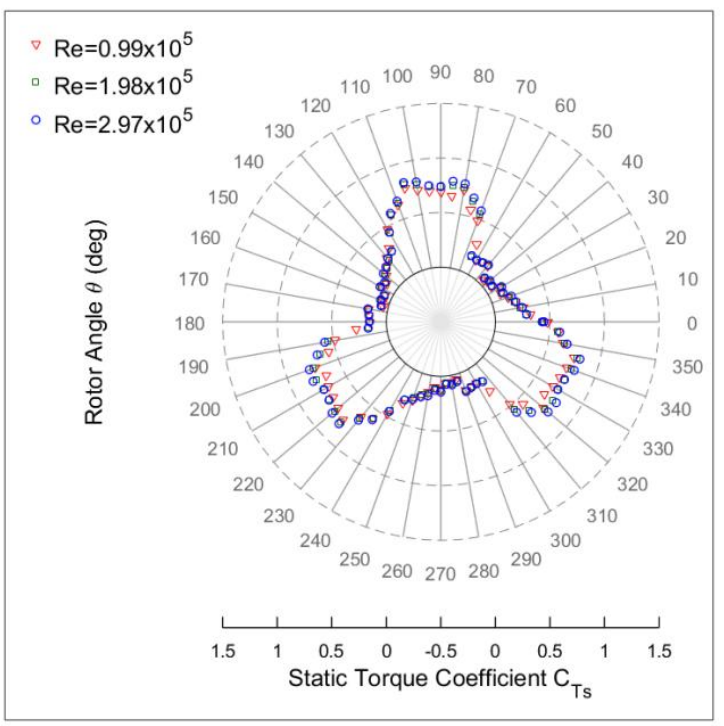

Fig.63 Variation of static torque coefficients at different rotor angles at different Reynolds numbers for harvester I. 
Effect of removing end plates on static performance of the energy harvester is also numerically studied. The distribution of static torque coefficient of harvester I is shown in Fig.63. The maximum static torque coefficient of harvester I occurs at rotor angle of $100^{\circ}, 220^{\circ}$ and $340^{\circ}$, while the minimum static torque coefficient takes place at $45^{\circ}, 165^{\circ}$ and $285^{\circ}$. The static torque coefficient is all positive throughout the whole revolution, at any rotor angle. This makes the energy harvester more easily to self-start rotation, and this is confirmed by low experimental cut-in Reynolds number. The static torque coefficient is not as positive as that of harvester $\mathrm{H}$, and the experimental cut-in Reynolds number is slightly higher than that of harvester $H$. The mechanism of improving the static performance of harvester $\mathrm{I}$ is similar to that of harvester $\mathrm{H}$, the additional air pathways created by removing end plates reduces the undesirable pressure built-up at convex sides of the blades and the torque in counter-rotation direction.

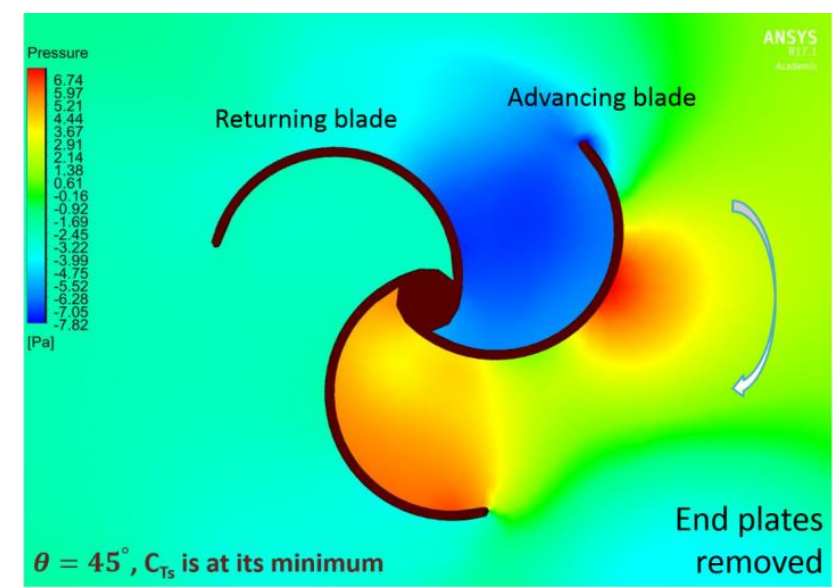

Fig.64 Pressure distribution at minimum static torque coefficient value for energy harvester $\mathbf{I}$. 
The pressure distribution pattern for harvester $I$ is also examined in Fig.64. As harvester I has three blades connected by solid shaft at the center, the pressure distribution pattern is similar to that of harvester A. However, as end plates at both ends of energy harvester are removed, air mass can move to downstream more easily at the sides. The pressure difference between sides of blades is reduced, especially at the advancing blade. The torque in counter-rotation direction is reduced, thus the cut-in Reynolds number is reduced.

Numerical dynamic performance study of harvester I gives us its characteristic curve, as shown in Fig.65. The power coefficient at a given tip speed ratio for harvester I is lower than that of harvester A. This means removal of end plates significantly reduces the torque generated on the energy harvester, therefore the power coefficient is also lower. The removal of end plates also leads smaller rotation speed can be practically achieved. Combined with smaller power coefficient, the efficiency of energy harvester I is much lower than that of harvester A.

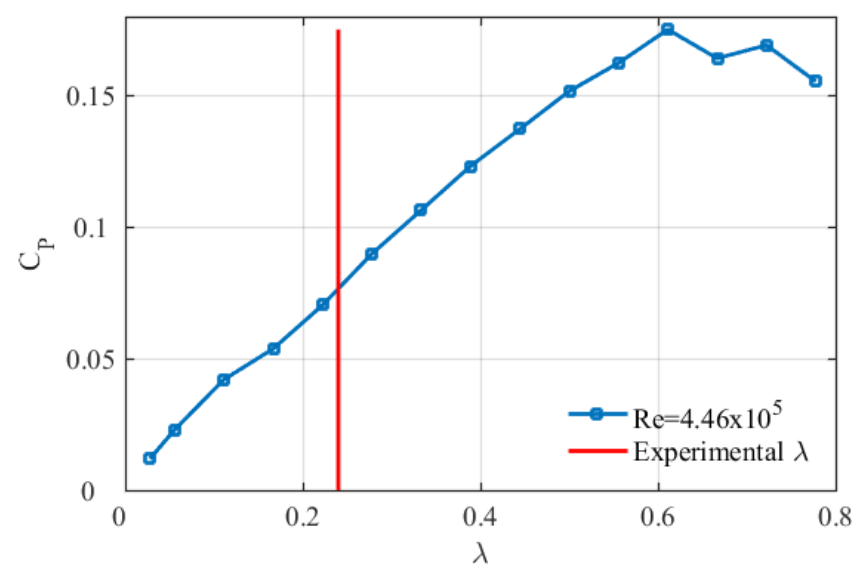


Fig.65 Performance characteristic curves for harvester I at $\mathrm{Re}=4.46 \times 10^{5}$. (The blue line indicates values attained by numerical work and the red line indicates experimentally achieved tip speed ratio).

\subsubsection{Effect of the energy harvester orientation}

Energy harvester $\mathbf{J}$ is used to study the effect of harvester installation orientation. The geometric dimension and structure of harvester $\mathbf{J}$ are exactly the same as harvester A. harvester A is installed in a way that it is rotating 'clockwise' in positive $\mathrm{z}$ direction (the concave side of blades facing the oncoming air flow is located at lower half of the harvester). Different from harvester A, harvester J has reversed orientation that it rotates 'anti-clockwise' in positive $\mathrm{z}$ direction (the concave side of blades facing the oncoming air flow is located at upper half of the harvester).

The cut-in Reynolds number of harvester $\mathrm{J}$ is relatively high, it is $8.32 \times 10^{5}$. The operational Reynolds number range is wider than harvester A, as harvester $\mathbf{J}$ can retain rotation at Reynolds number as low as $1.91 \times 10^{5}$. The experimental results of induced emf measurements are to be found in Appendix B. As show in Fig.66, rotation speed and electrical power output both increase with increased Reynolds number. The tendency of 'saturation' in rotation speed is observed at high Reynolds number as shown in Fig.66a, and this leads to reduction in overall energy conversion efficiency as indicated in Fig.66d. 

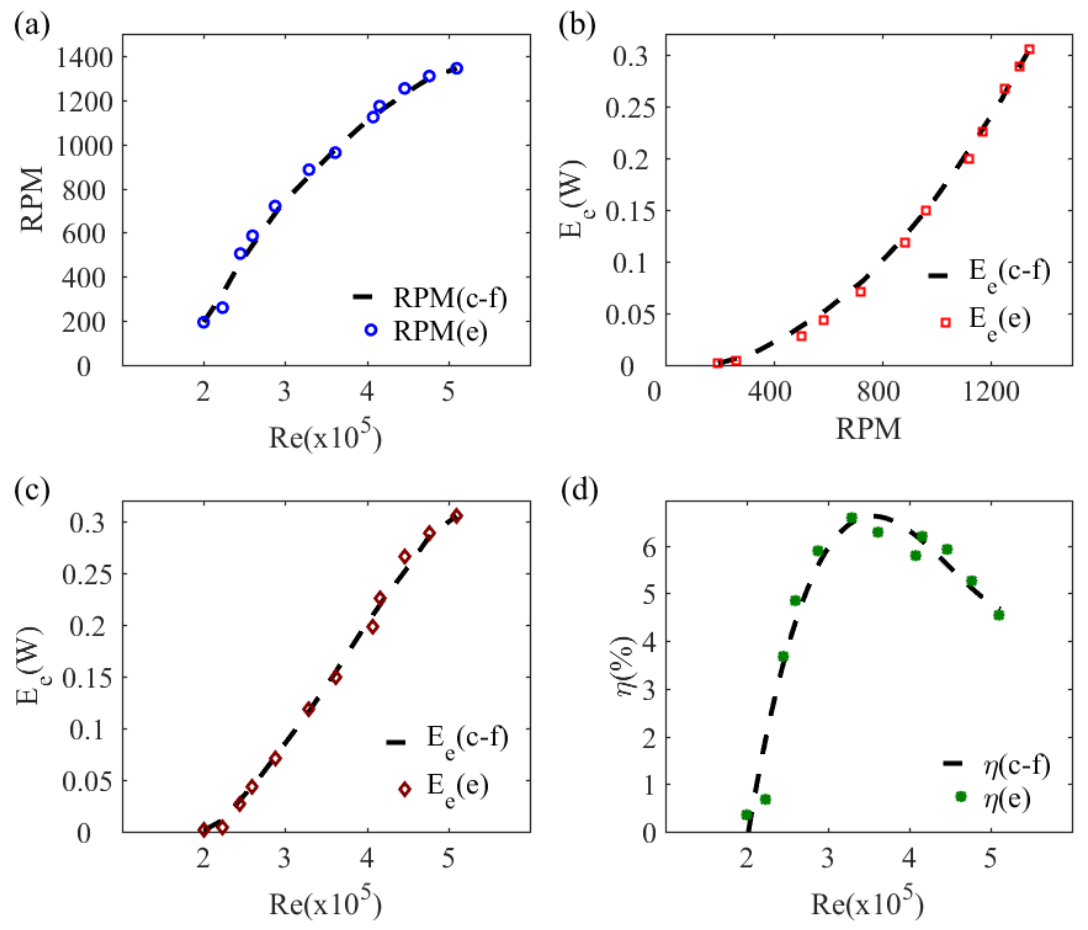

Fig.66 (a) RPM variation with Reynolds number. (b) Electrical power output varied with RPM. (c) Electrical power and (d) Overall energy conversion efficiency varied with Reynolds number for energy harvester J. (c-f denotes curve fitting and e denotes experimental results).

The energy conversion efficiencies of energy harvester with different installation orientations are compared in Fig.67. It can be clearly seen that energy harvester installed in 'anti-clockwise' orientation is associated with higher overall energy conversion efficiency $\left(\eta_{\max }=6.59 \%\right)$. The reason for this observation is that the energy harvester is installed on a step height platform, and interaction between the step height platform and the energy harvester leads to difference in energy conversion efficiency with different installation orientations. As the step height platform underneath the energy harvester blocks the air flow, the direction of air flow is directed upward. When the energy harvester is installed in 'anti-clockwise' orientation, it is able to better utilize air flows from both horizontal main flow 
direction and the upward flow from step height platform edge. In this way, greater pressure difference between sides of blade is achieved as more air mass is captured between harvester blades. On the other hand, this increment in pressure difference also leads to higher cut-in Reynolds number, as the counter-rotation torque generated is larger.

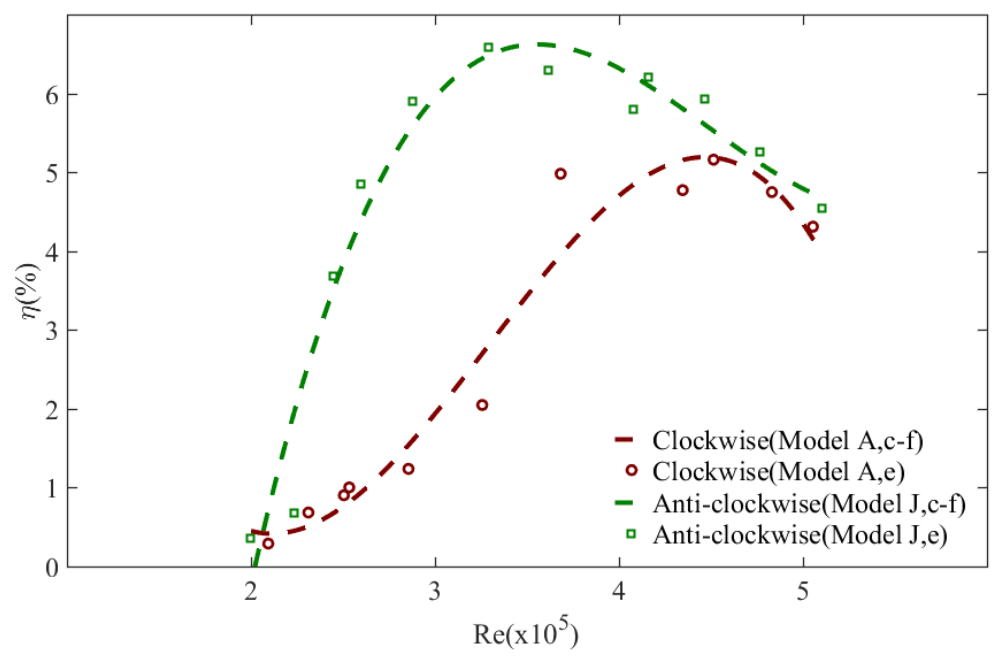

Fig.67 Comparison of overall energy conversion efficiency from harvester $A$ and $J$ installed in different orientations, as the Reynolds number is increased. (c-f denotes curve fitting and e denotes experimental results).

The static performance of harvester $\mathbf{J}$ is numerically studied and distribution of static torque coefficient is obtained as in Fig.68. The pattern of the distribution is different from that of harvester A. The positive static torque coefficient is observed in rotor angle ranges of $0^{\circ}-55^{\circ}, 90^{\circ}-175^{\circ}, 210^{\circ}-295^{\circ}$ and $330^{\circ}-360^{\circ}$. The maximum static torque coefficient values are found at rotor angle of $10^{\circ}, 130^{\circ}$ and $250^{\circ}$, while the minimum values are found at $70^{\circ}, 190^{\circ}$ and $310^{\circ}$. As the geometric structure of harvester $\mathbf{J}$ is the same as that of harvester $\mathrm{A}$, the magnitudes of static 
torque coefficients are similar. Therefore, the static performance of the two energy harvester models do not differ significantly.

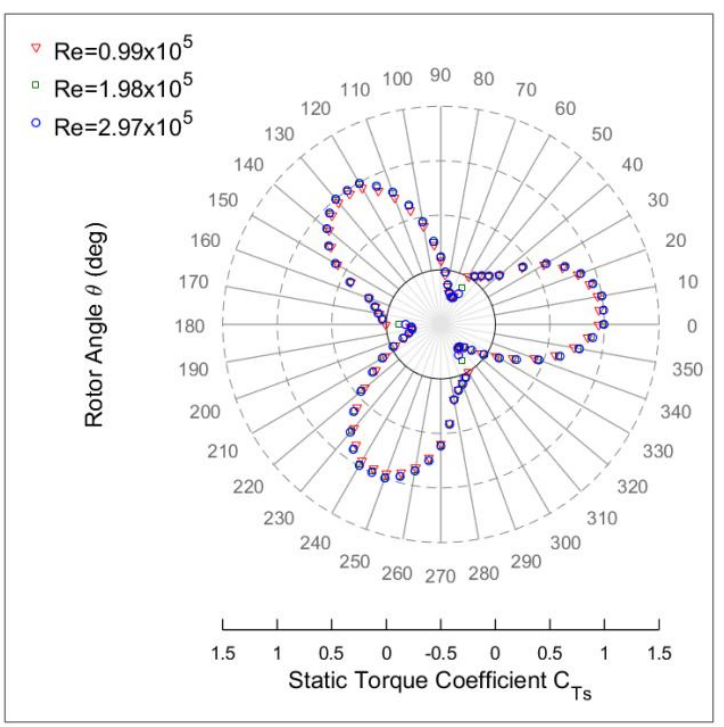

Fig.68 Variation of static torque coefficients at different rotor angles at different Reynolds numbers for harvester $\mathbf{J}$.

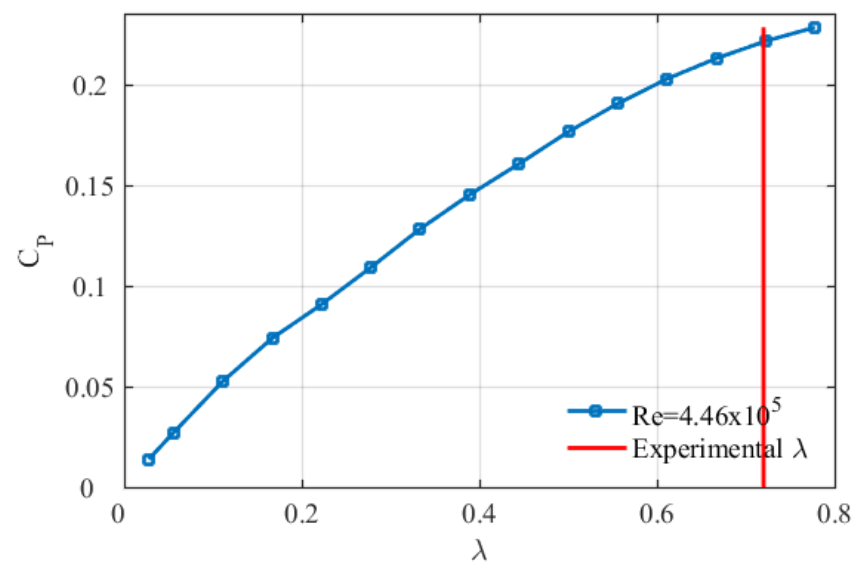

Fig.69 Performance characteristic curves for harvester $\mathrm{J}$ at $\mathrm{Re}=4.46 \times 10^{5}$. (The blue line indicates values attained by numerical work and the red line indicates experimentally achieved tip speed ratio). 
Dynamic study of harvester $\mathbf{J}$ gives its characteristic curve as shown in Fig.69. As harvester $\mathrm{A}$ and $\mathrm{J}$ are exactly the same in geometric structure and dimension, except the orientation, the characteristic curves of the two are similar. Compared to harvester $\mathrm{A}$, harvester $\mathrm{J}$ is able to utilize both the main flow and the upward flow from step height platform, the rotation speed achieved by harvester $\mathrm{J}$ is higher than that of harvester A. Therefore, the power coefficient attained by harvester $\mathrm{J}$ in practice is higher than that of harvester A. Energy harvester $\mathrm{J}$ has the highest power coefficient amongst all models in preliminary parametric study, and this is the same in wind tunnel tests in which proves harvester $\mathrm{J}$ is associated with the higher energy conversion efficiency.

\subsection{Concluding remarks}

The performances of 10 harvesters are summarized in Table 9. It can be seen that if the static performance is considered, the energy harvester $\mathrm{H}$ and I perform better, since they are associated with lower cut-in Reynolds numbers in comparison with those of other harvesters. If dynamic performance is concerned, harvester $\mathrm{J}$ is the best, as it is associated with the maximum energy conversion efficiency of $\eta_{\max }=6.59 \%$. Thus harvester $\mathrm{J}$ is the optimum design obtained from present systematic studies. To achieve better understanding of the effect of the harvester orientation, further investigations are conducted on a number of energy harvesters with different geometric structures installed in 'anti-clockwise' orientation. The results are presented and discussed in the following section. 
Table 9 Summary of performances of designed energy harvester models

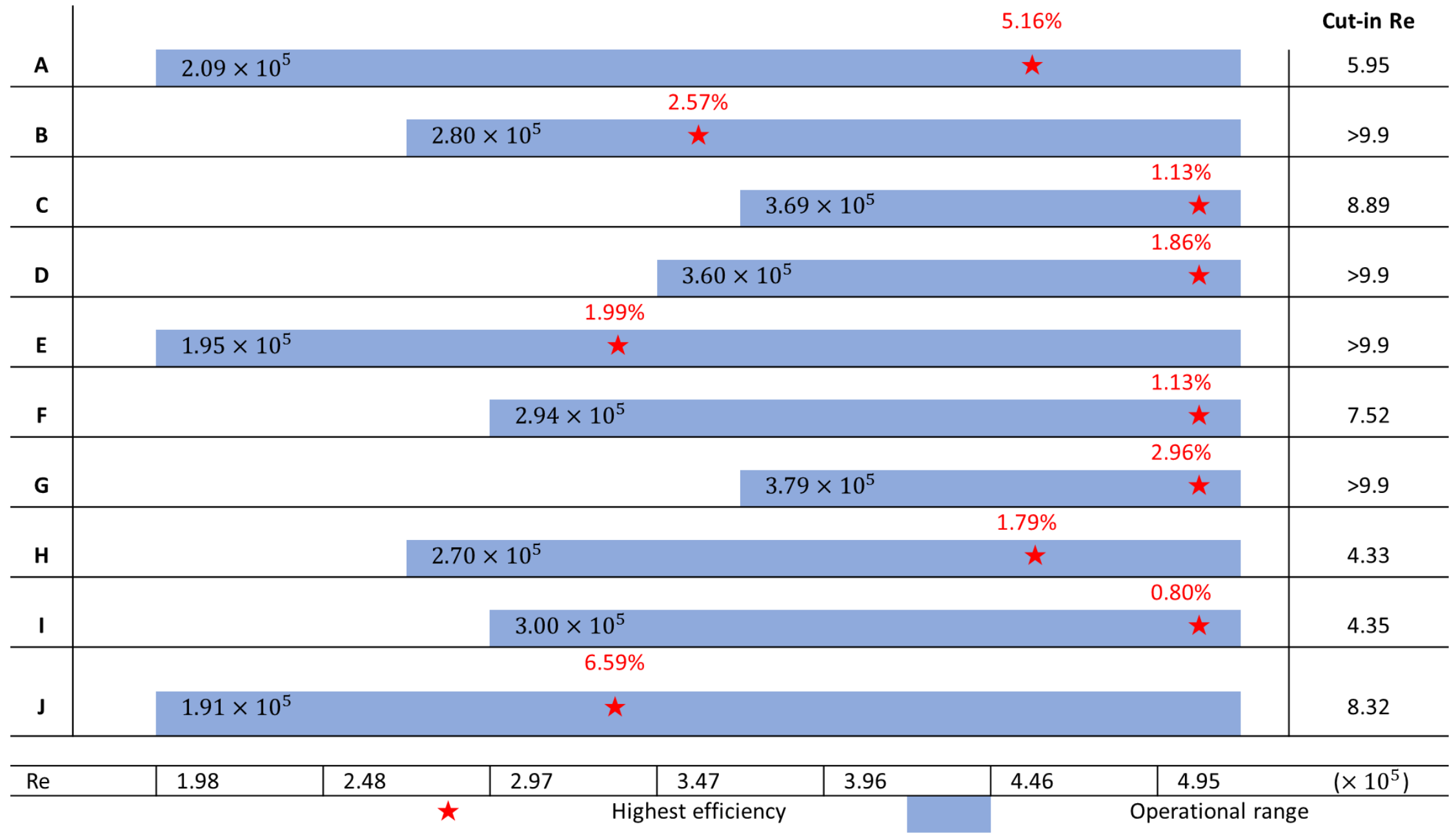




\subsection{Effect of the harvester orientation}

Based on understanding attained from previous parametric study, it is noticed that the energy harvester installation orientation will affect the energy conversion efficiency and the effect of interaction with step height platform should be considered. The benchmark energy harvester is installed with its concave side facing oncoming air flow at the lower half of the harvester ('clockwise' orientation). If the energy harvester is to be installed at the edge of a step height platform, there will be an upward flow air mass near the platform edge. And when the energy harvester orientation is reversed (harvester $\mathrm{J}$ ), pressure is better concentrated, as 'anti-clockwise' orientated harvester model utilizes both upward flow from step height platform edge and main flow in favor of its rotation direction (Fig.70).

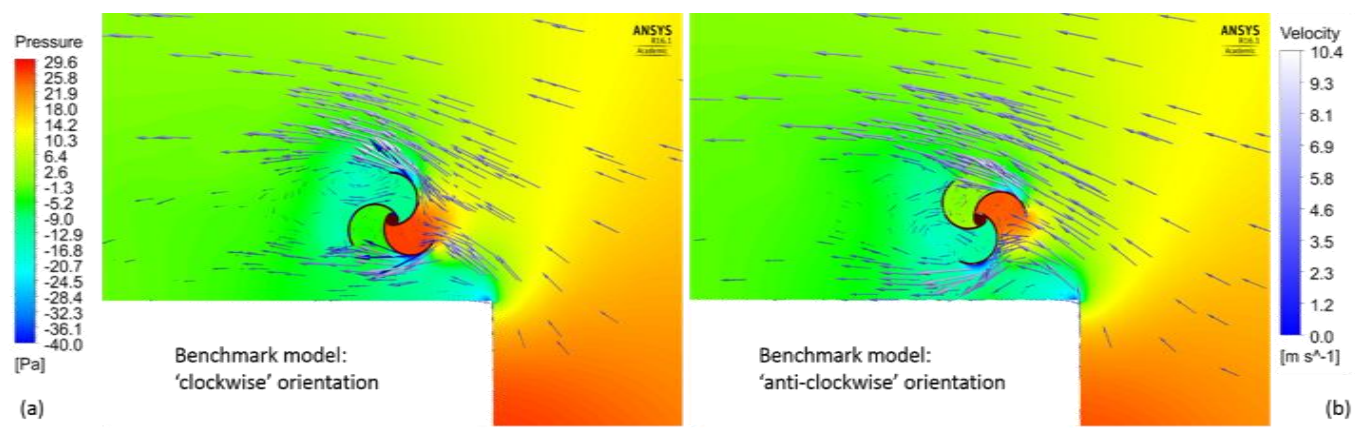

Fig.70 Pressure distribution and velocity vector diagram of benchmark energy harvester model with (a) 'clockwise' orientation and (b) 'anti-clockwise' orientation.

Experimentally, reversing installation orientation of harvester A leads to increment in rotation speed of energy harvester (see Fig.71). Consequently, as previously presented, the energy conversion efficiency is increased from 5.16\% (harvester A) to $6.59 \%$ (harvester J), by changing installation orientation. 


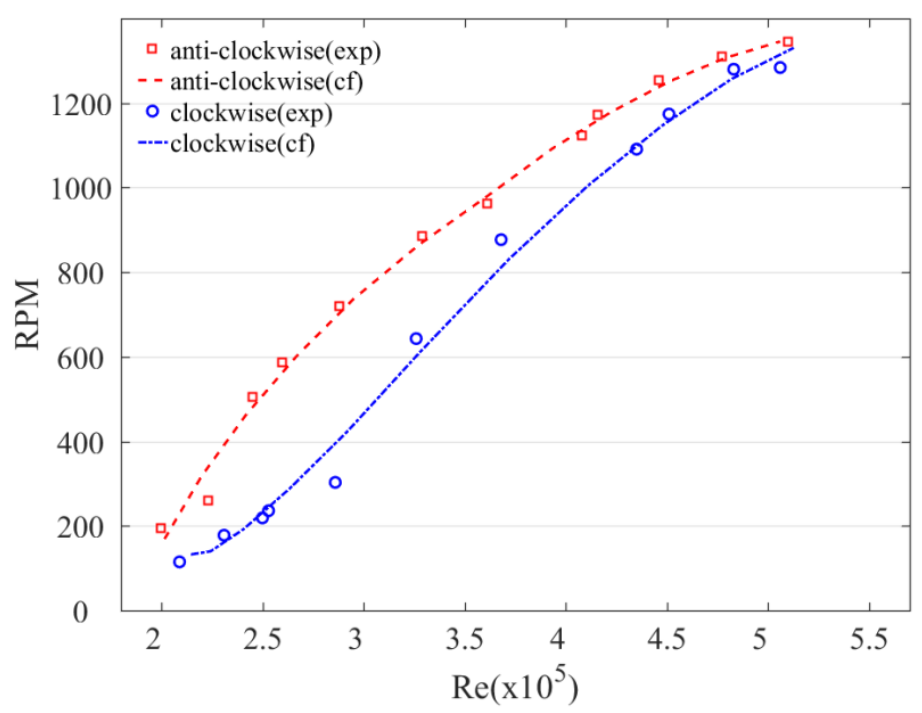

Fig.71 Variation of RPM with Re for 'clockwise'(harvester A) and 'anticlockwise'(harvester J) installation orientation. ('exp' denotes experimental data and 'cf' denotes curve fitting line)

Fig.72 is showing the pressure distribution near the benchmark energy harvester model installed in 'clockwise' and 'anti-clockwise' orientations, at rotor angles where instantaneous dynamic torque coefficient is at its minimum and maximum values, respectively. It can be seen that when the energy harvester is installed in 'clockwise' orientation, the opening between the blades does not match perfectly with air flow directions. At $\mathrm{C}_{\mathrm{TdMAX}}$ rotor angle, the returning blade is blocking part of upward airflow, hence generating counter-rotation torque. At $\mathrm{C}_{\text {TdMIN }}$ rotor angle, airflows in both directions are directed to the convex side of the blade, generating great pressure difference between two sides of advancing blade and larger torque in counter-rotation direction. When harvester orientation is changed, the opening between blades can face both airflow directions and generate larger $\mathrm{C}_{\mathrm{TdMAX}}$ and torque in rotation direction. Even when the harvester is at $\mathrm{C}_{\text {TdMIN }}$ rotor angle, the pressure difference at returning blade responsible for generation of counter-rotation 
torque is smaller, as only upward airflow is directed to the convex side of returning blade. Therefore, $\mathrm{C}_{\mathrm{TdMAX}}$ for 'anti-clockwise' orientated benchmark model is more positive while $\mathrm{C}_{\text {TdMIN }}$ is less negative. Both are desirable to produce higher rotation speed and energy conversion efficiency.

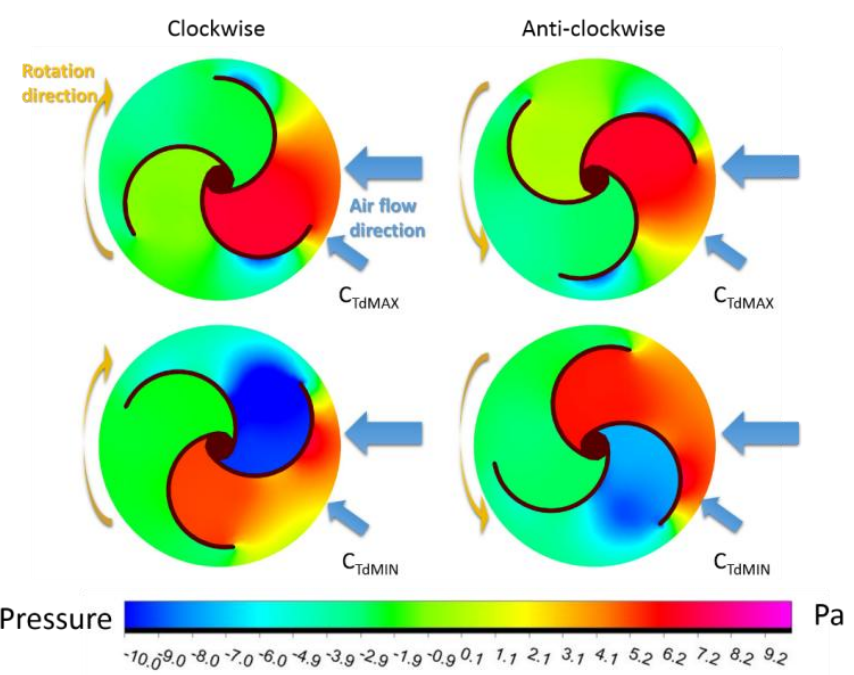

Fig.72 Pressure distribution at $R e=1.49 \times 10^{5}$ for benchmark energy harvester with 'clockwise' and 'anti-clockwise' installation orientation

Here another parametric study is conducted to investigate the combined effect of energy harvester installation orientation and its geometric structure. The energy harvester models being studied are harvester B to I, covering geometric structure design factors: 1) number of energy harvester blade number $(\mathrm{N}), 2)$ geometric size of energy harvester (SR), 3) aspect ratio of energy harvester (AR), 4) type of central part and 5) end plates. The parametric study is experimentally done by installing energy harvesters as described in Fig.70 (denoted as 'clockwise' in z direction if the concave blade facing oncoming air flow is at lower half of the harvester as Fig.70a, and denoted as 'anti-clockwise' in $\mathrm{z}$ direction of the concave blade is at 
upper half of the harvester as Fig.70b), and measuring their rotation speed at different Reynolds numbers. Additionally, the pressure distribution around the energy harvester at $R e=1.49 \times 10^{5}$ is obtained from numerical study. Special attention is paid to study the pressure distribution when instantaneous dynamic torque coefficient is at its maximum value $\left(\mathrm{C}_{\mathrm{TdMAX}}\right)$ and minimum value $\left(\mathrm{C}_{\mathrm{TdMIN}}\right)$. The experimental results of wind tunnel tests on energy harvester models installed in 'anti-clockwise' orientation can be found in Appendix C.

\subsubsection{Combined effect of energy harvester orientation and number of blades $(N)$}

Comparison of rotation speed attained at different Reynolds numbers by two-blade model (harvester B) installed with different orientations can be seen in Fig.73a. It can be seen that at a given Reynolds number, higher rotation speed can be attained when the energy harvester is installed in 'anti-clockwise' orientation, as compared to the one in 'clockwise' orientation. However, as shown in Fig.73b, changing installation orientation does not create higher rotation speed for harvester $\mathrm{C}$ with four blades. Whether the energy harvester is able to capture the upward air flow from the step height platform edge will determine enhancement effect of changing installation orientation. As compared to benchmark model with three blades, twoblade energy harvester has larger opening for oncoming air to approach while fourblade harvester has much narrower opening. Wider opening allows air reach the concave side of the blade more easily, and greater number of blades may hinder air 
entrance path towards the harvester center. Greater details are revealed from pressure distribution patterns around the energy harvesters.

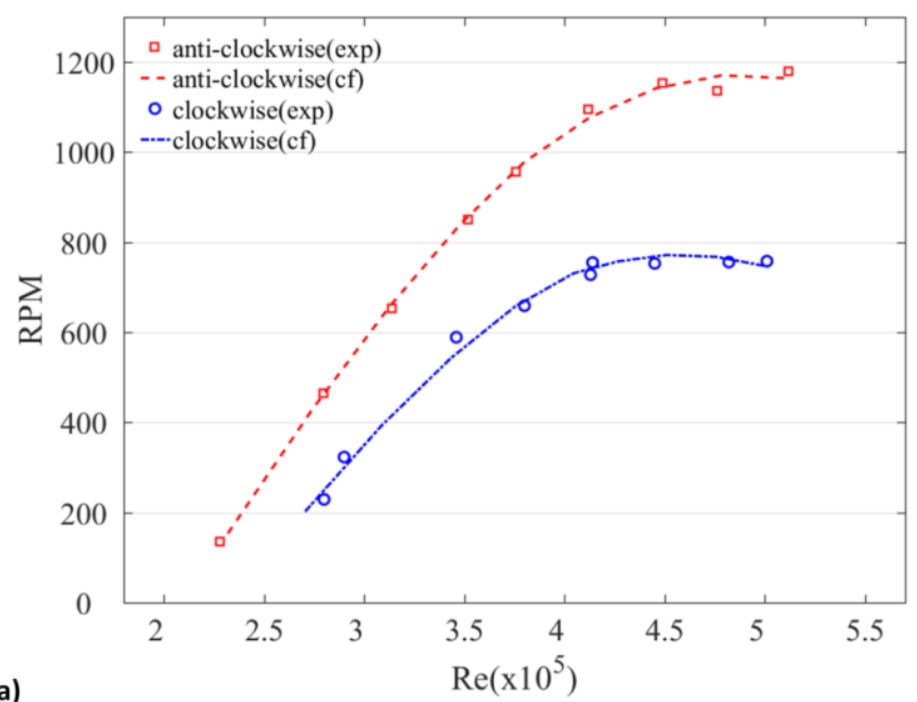

(a)

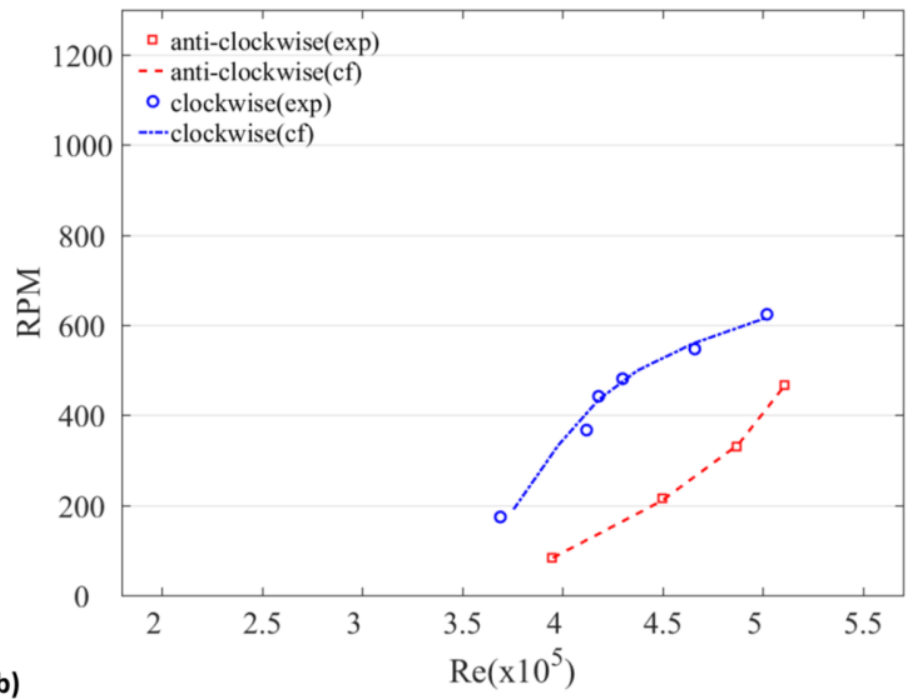

Fig.73 Variation of RPM with Re for (a) harvester B and (b) harvester C with 'clockwise' and 'anti-clockwise' installation orientation. ('exp' denotes experimental data and 'cf' denotes curve fitting line)

Fig.74 is showing the numerically obtained instantaneous $C_{T d}$ variation within one revolution for harvester $\mathrm{B}$ and $\mathrm{C}$ at $\mathrm{Re}=1.49 \times 10^{5}$ and the angular velocity of 
the harvester models is $\omega=5 \mathrm{rad} / \mathrm{s}$. For energy harvester B, 'clockwise' orientation is associated with higher $\mathrm{C}_{\text {TdMAX }}$ and lower $\mathrm{C}_{\text {TdMIN }}$ values. The differences in $\mathrm{C}_{\text {TdMAX }}$ and $\mathrm{C}_{\mathrm{TdMIN}}$ values between two orientations are not significant. It seems that when $\mathrm{C}_{\text {TdMAX }}$ values are close, positive or less negative $\mathrm{C}_{\text {TdMIN }}$ value will generate higher rotation speed of the harvester model. For harvester $\mathrm{C}$, 'clockwise' orientation possesses higher $\mathrm{C}_{\mathrm{TdMAX}}$ and $\mathrm{C}_{\mathrm{TdMIN}}$ values, especially its $C_{\text {TdMAX }}$ value is significantly higher than that of 'anti-clockwise' orientation. Hence, 'clockwise' orientated energy harvester $\mathrm{C}$ is associated with higher rotation speed than the one in opposite orientation.

Fig.75 is showing the comparison of pressure distribution patterns around harvester $\mathrm{B}$ and $\mathrm{C}$ in 'clockwise' and 'anti-clockwise' orientations. For harvester B with two blades, wide opening between two blades allows easier capture of airflows. With 'clockwise' orientation, at $\mathrm{C}_{\mathrm{TdMAX}}$ rotor angle, air flows from both directions are captured, creating high pressure at concave side of the blade. At $\mathrm{C}_{\text {TdMIN }}$ rotor angle, airflows are directed to convex side of the blade, creating strong torque in counterrotation direction. At this rotor angle, the pressure difference between the sides of advancing blade is significant, thus $C_{\mathrm{Td}}$ is more negative. When the installation orientation is reversed, the increment in rotation speed is achieved as $\mathrm{C}_{\mathrm{Td}}$ is less negative. At $\mathrm{C}_{\text {TdMIN }}$ rotor angle, the pressure difference between sides of the returning blade is smaller, as part of the airflows is directed towards the concave side of the advancing blade. This leads to smaller torque generated in counterrotation direction throughout the whole revolution, therefore higher rotation speeds are attained. However, it must be noted that the rotor angle ranges of positive and 
negative $C_{T d}$ values are very distinct, which means the variation in $C_{T d}$ value is large. This large variation in $\mathrm{C}_{\mathrm{Td}}$ value is undesirable for continuous high rotation speed to be achieved.
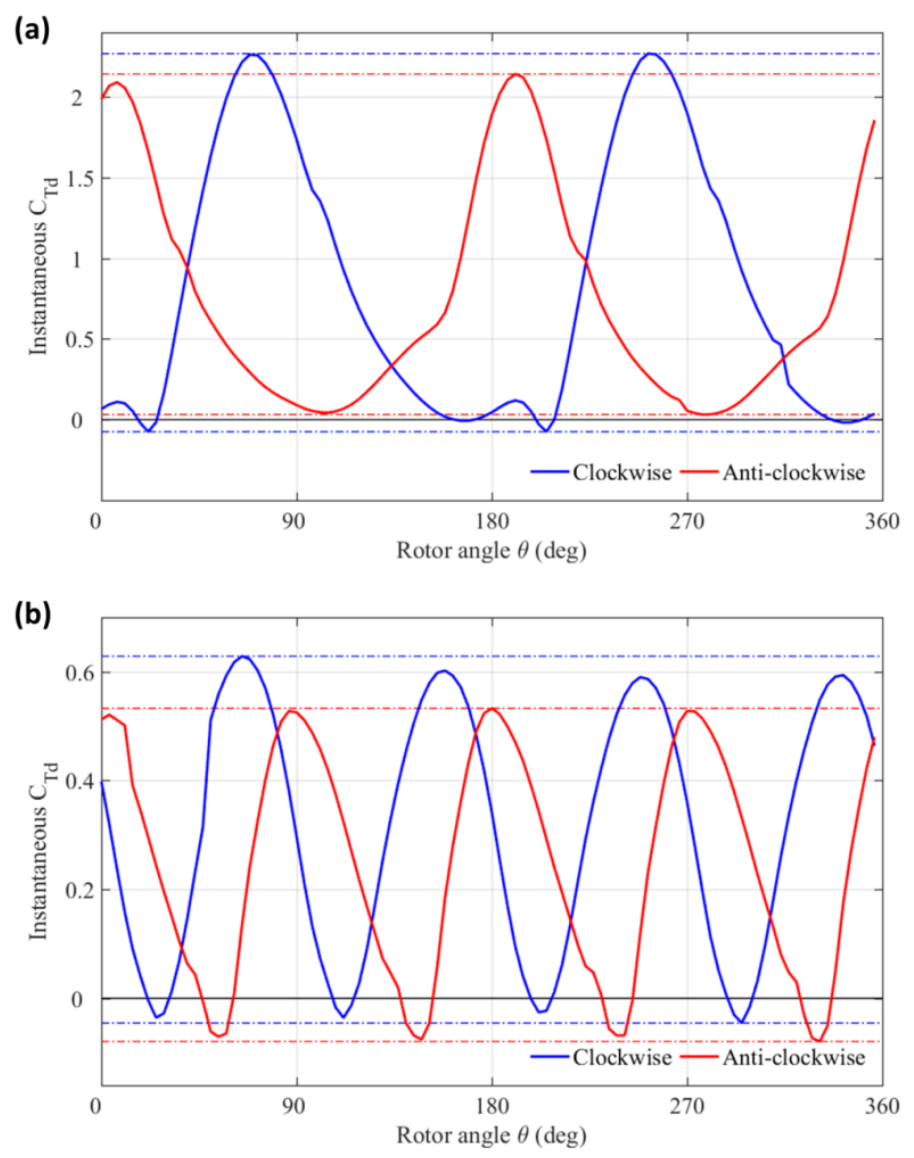

Fig.74 Instantaneous $\mathbf{C}_{\mathrm{Td}}$ within one revolution for energy harvester (a) B and (b) $\mathrm{C}$ at $\operatorname{Re}=1.49 \times 10^{5}$ and $\omega=5 \mathrm{rad} / \mathrm{s}$. 
(a)
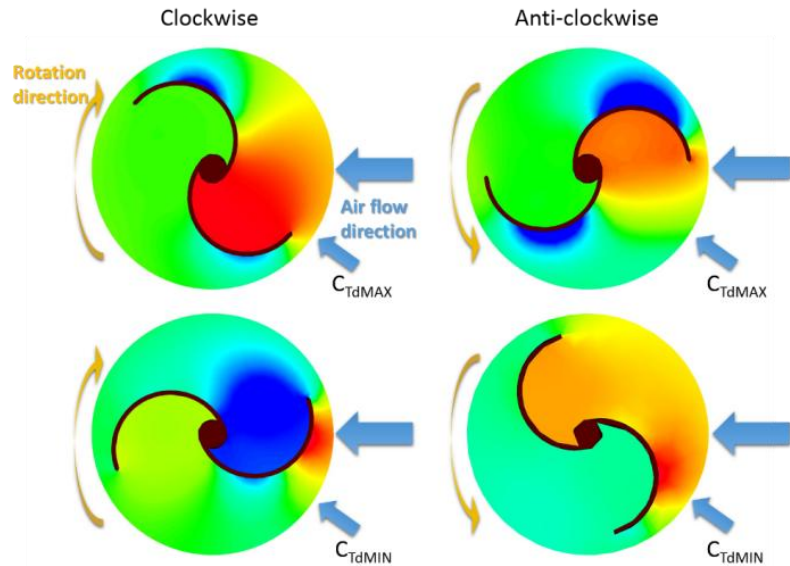

(b)
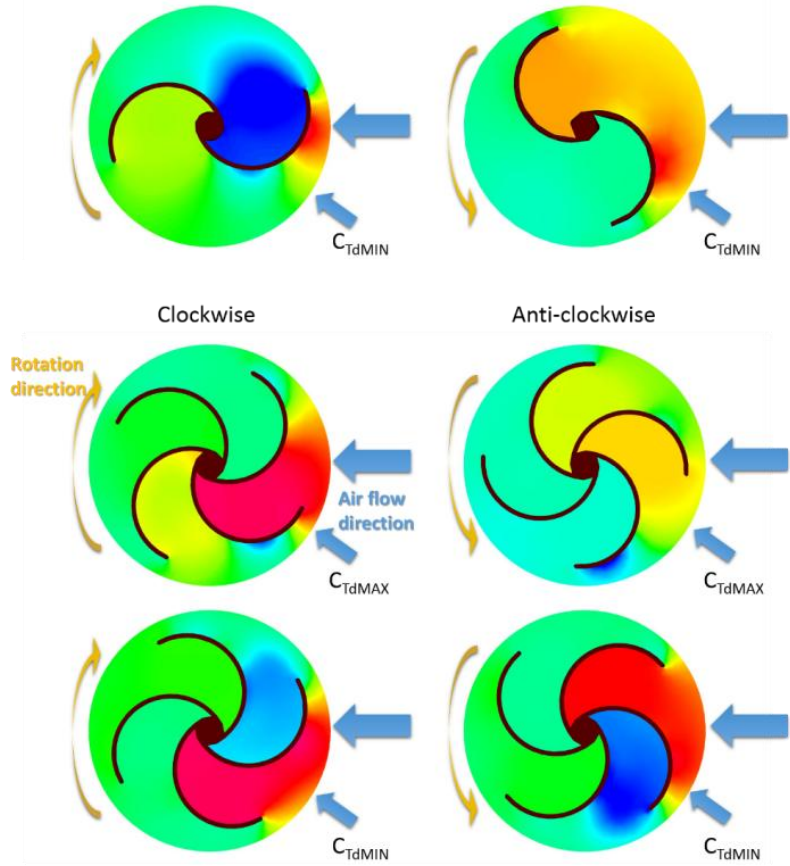

Pressure

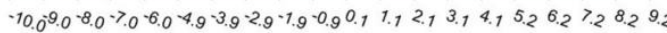

Fig.75 Pressure distribution at $R e=1.49 \times 10^{5}$ for energy harvester (a) $B$ and (b) $C$ with 'clockwise' and 'anti-clockwise' installation orientation.

For the four-blade harvester, less distinct $\mathrm{C}_{\mathrm{TdMAX}}$ and $\mathrm{C}_{\mathrm{TdMIN}}$ rotor angles can be seen. This is due to the fact at high pressure region constrained between both concave and convex sides of two adjacent blades will generate comparable torques in both rotation direction and counter-rotation. Hence, compared to two- and threeblade energy harvesters, harvester $\mathrm{C}$ is associated with lower rotation speed. When harvester $\mathrm{C}$ is in 'anti-clockwise' orientation, the pressure at concave side of blade is lower, and $\mathrm{C}_{\text {TdMAX }}$ is less positive. At $\mathrm{C}_{\mathrm{TdMIN}}$ rotor angle, the high pressure at convex side of returning blade generates larger counter-rotation torque, and $\mathrm{C}_{\text {TdMIN }}$ 
is more negative. Both less positive $\mathrm{C}_{\text {TdMAX }}$ and more negative $\mathrm{C}_{\text {TdMIN }}$ are undesirable for high rotation speed to be achieved.

\subsubsection{Combined effect of energy harvester orientation and geometric size} $(S R)$

Effects of changing energy harvester installation orientation for harvester D $(\mathrm{SR}=2 / 3)$ and $\mathrm{E}(\mathrm{SR}=4 / 3)$ can be seen from Fig.76.For smaller-size energy harvester, the space for pressure to build up is smaller and the harvester rotates at lower rotation speed. Also, smaller harvester has shorter torque arm, thus smaller amount of torque can be generated. This shorter torque arm also makes the energy harvester less effective in capturing upward airflow from step height edge when it is in 'anti-clockwise' orientation. Therefore, the smaller energy harvester is not able to take advantage of 'anti-clockwise' orientation, as shown in Fig.76a. Together with harvester A, it can be seen that for larger energy harvesters with larger available space between blades, it is easier to develop a high-pressure region at concave side of blade. The larger energy harvester is associated with longer torque arm, which improves energy conversion efficiency in two ways: larger torque can be generated and it is easier to capture upward air flow. However, the enhancement effect of reversing installation orientation is not as significant as expected (Fig.76b). This can possibly be explained by larger harvester's greater weight and greater friction between harvester's shaft and its support. For the same reason, the larger energy harvester does not attain higher rotation speed than the 
benchmark model, neither with 'clockwise' orientation nor with 'anti-clockwise' orientation.

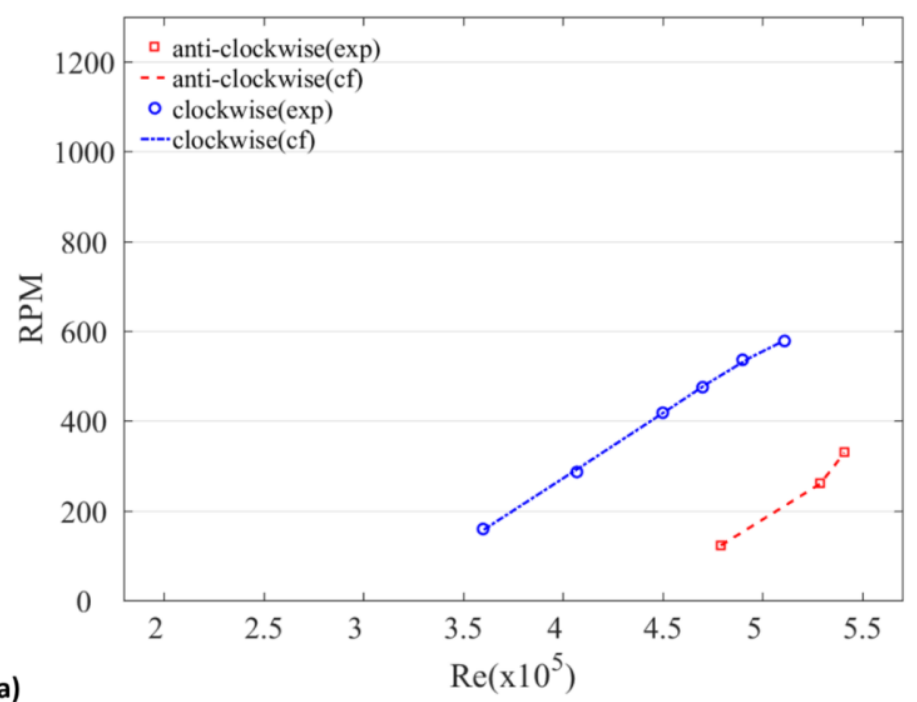

(a)

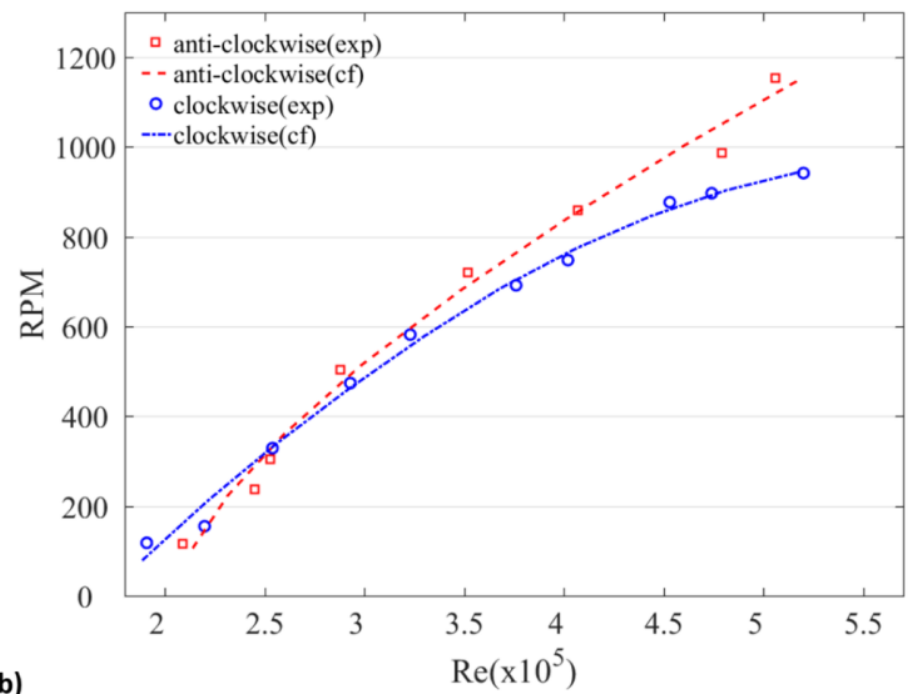

Fig.76 Variation of RPM with Re for harvester (a) D and (b) E with 'clockwise' and 'anti-clockwise' installation orientation. ('exp' denotes experimental data and 'cf' denotes curve fitting line)

From Fig.77 which is showing the numerically obtained instantaneous $C_{T d}$ variation within one revolution for harvester $\mathrm{D}$ and $\mathrm{E}$, insights on differences in 
rotation speeds of two harvesters can be attained. For energy harvester D, its 'clockwise' orientation has both higher $\mathrm{C}_{\mathrm{TdMAX}}$ and less negative $\mathrm{C}_{\mathrm{TdMIN}}$, therefore its rotation speed is higher when installed in 'clockwise' orientation. For harvester $\mathrm{E}$, the 'anti-clockwise' orientation has higher and more desirable $\mathrm{C}_{\mathrm{TdMAX}}$, but $\mathrm{C}_{\text {TdMIN }}$ is more negative. The resultant effect leads to similar rotation speeds of 'clockwise' and 'anti-clockwise' orientated harvester E.
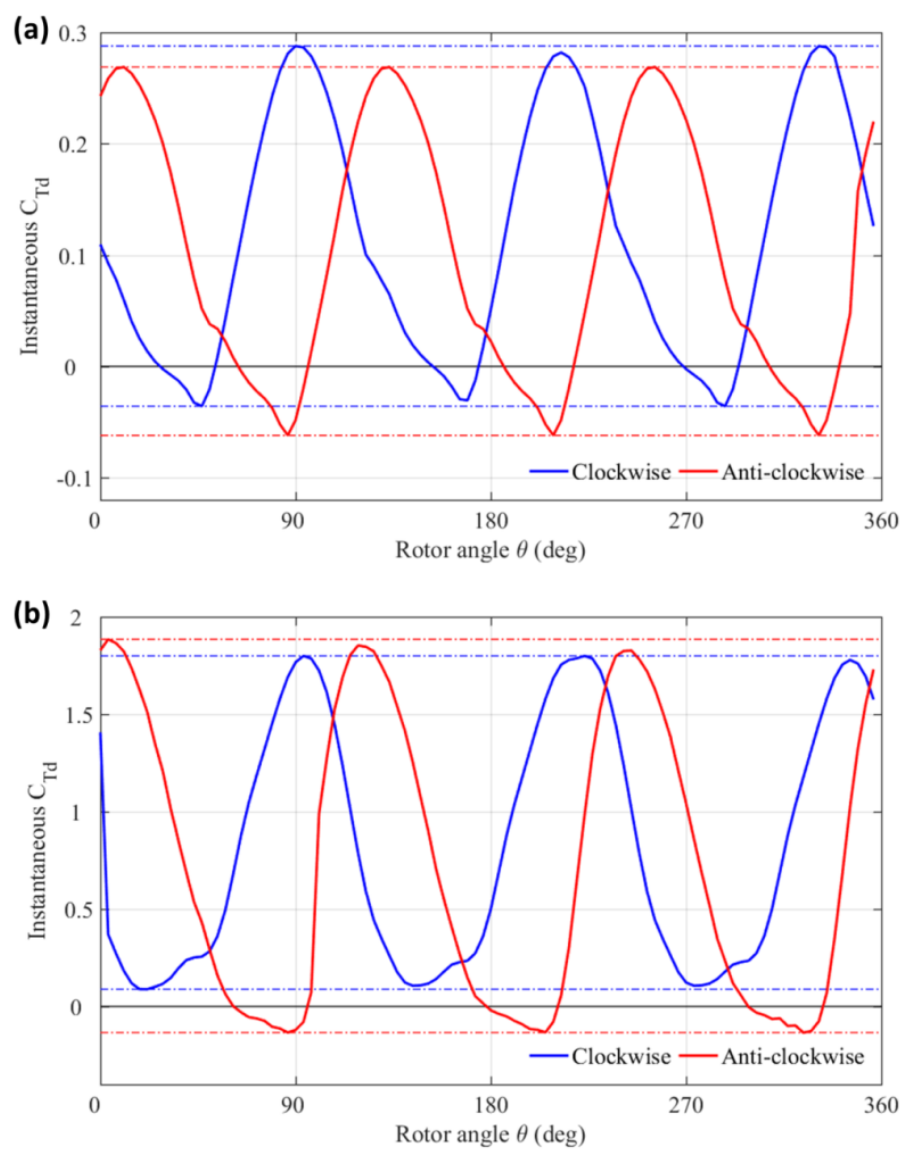

Fig.77 Instantaneous $C_{T d}$ within one revolution for energy harvester (a) $D$ and (b) $E$ at $\operatorname{Re}=1.49 \times 10^{5}$ and $\omega=5 \mathrm{rad} / \mathrm{s}$ 
(a)
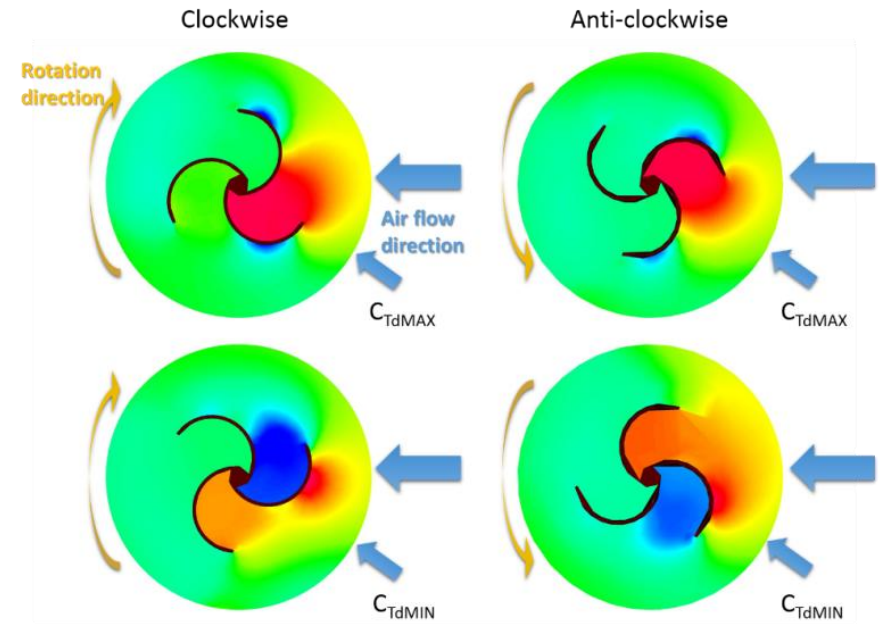

(b)
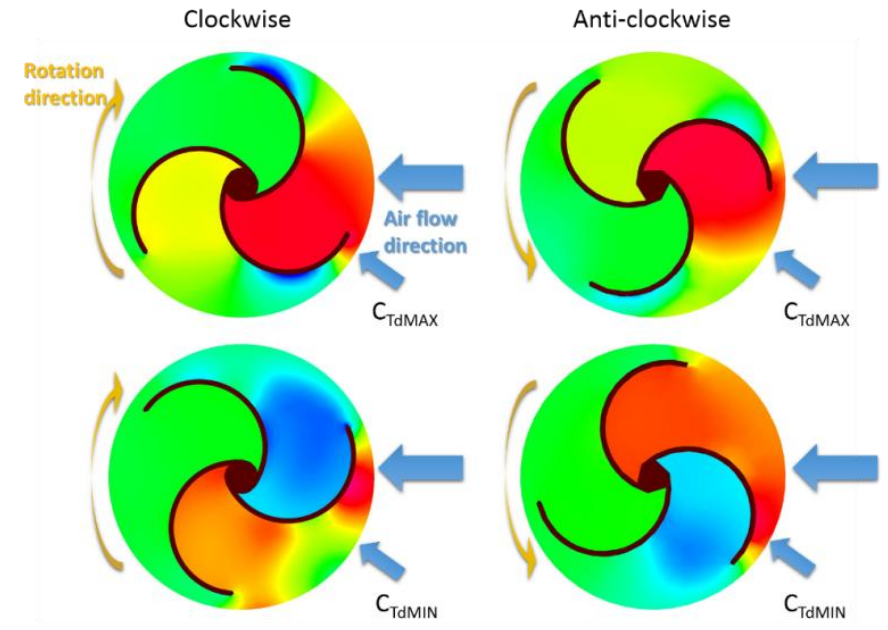

Pressure

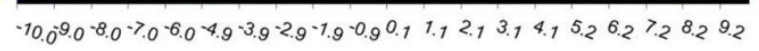

Fig.78 Pressure distribution at $R e=1.49 \times 10^{5}$ for energy harvester (a) D and (b) E with 'clockwise' and 'anti-clockwise' installation orientation.

Pressure distributions of harvester D and E installed in 'clockwise' and 'anticlockwise' orientations are shown in Fig.78. It can be seen that the pressure distributions are very similar, which include the distribution pattern as well as the rotor angles at which $\mathrm{C}_{\text {TdMAX }}$ and $\mathrm{C}_{\text {TdMIN }}$ take place. The geometric size of the energy harvester affects its rotation speed in two ways: firstly, the geometric size determines the torque arm length of the harvester, thus affects the torque generated; 
secondly, the geometric size determines the size of space available between harvester blades for high pressure to be built up. Smaller energy harvester has shorter torque arm length and smaller space between blades, attaining smaller rotation speed. As energy harvester shaft is fixed at the same height and the same distance away from the step height edge, smaller energy harvester blade tip is further away from the step height edge thus is less effective in capturing upward airflow from step height platform. As both $\mathrm{C}_{\text {TdMAX }}$ and $\mathrm{C}_{\text {TdMIN }}$ are lower than the 'clockwise' one, the smaller energy harvester does not better utilize 'anti-clockwise' orientation. Additionally, it suggests the need to optimize energy harvester's installation location according to its geometric size.

Moreover, it is noticed that the enhancement effect of 'anti-clockwise' orientation on harvester $\mathrm{E}$ is limited, as longer torque arm length leads to both higher $\mathrm{C}_{\mathrm{TdMAX}}$ and lower $\mathrm{C}_{\mathrm{TdMIN}}$. Practical reasons for limited enhancement effect of 'anticlockwise' orientation include the greater weight and greater friction between harvester's shaft and its support contributed by the larger harvester size. For the same reason, the larger energy harvester does not attain higher rotation speed than the benchmark model, neither with 'clockwise' orientation nor with 'anti-clockwise' orientation. 


\subsubsection{Combined effect of energy harvester orientation and aspect ratio (AR)}

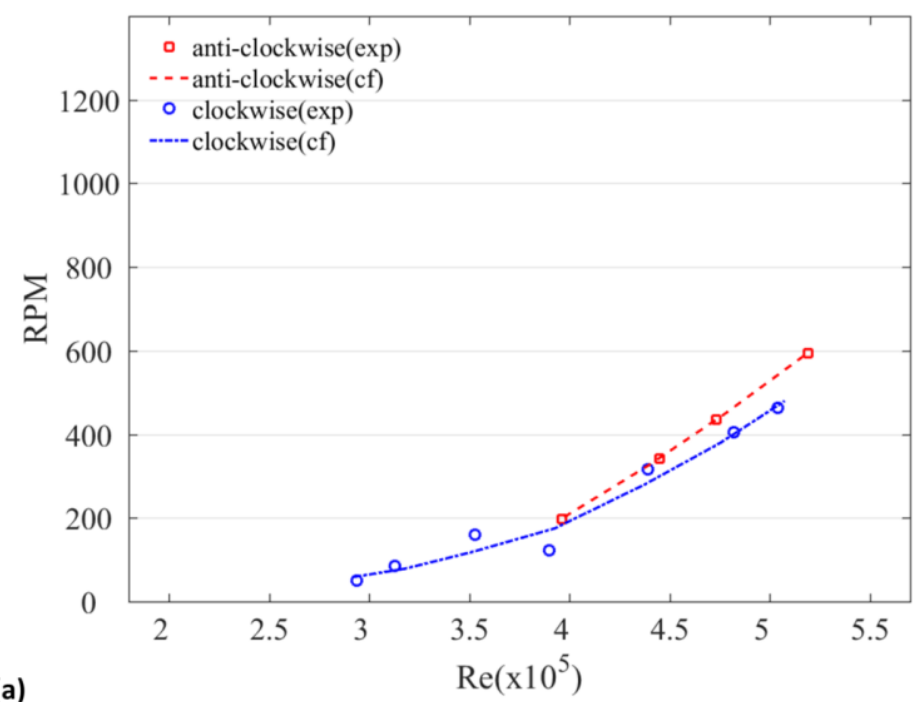

(a)

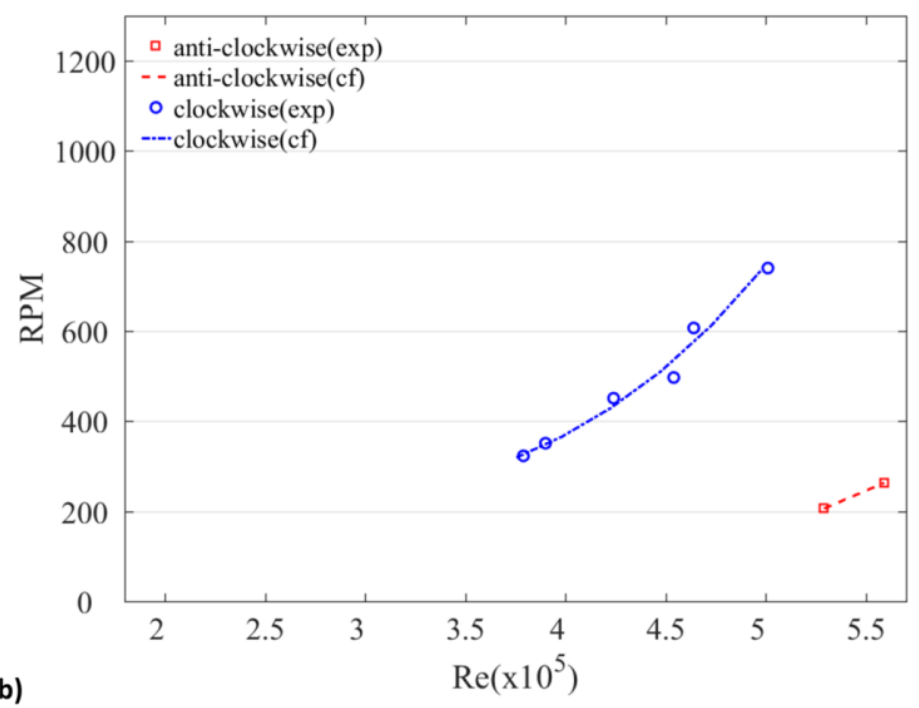

Fig.79 Variation of RPM with Re for harvester (a) F and (b) G with 'clockwise' and 'anti-clockwise' installation orientation. ('exp' denotes experimental data and 'cf' denotes curve fitting line)

Effect of changing energy harvester orientation on harvester $F$ and $G$ with different aspect ratios is shown in Fig.79. For energy harvester with $A R=1 / 2$, it can be see that although the harvester diameter and torque arm length is unchanged, there is smaller space available for pressure to build up as the energy harvester length is 
reduced. This smaller space makes the energy harvester less effective in capturing upward airflow when it is in 'anti-clockwise' orientation. In Fig.79a, it can be seen that the 'anti-clockwise' orientation does not increase the rotation speed of the harvester. Energy harvester with $\mathrm{AR}=2$ less effectively utilizes 'anti-clockwise' orientation for the same reason as previously discussed for energy harvester with smaller geometric size. This is due to shorter torque arm length reduces total amount of torque can be generated, and cannot capture the upward airflow effectively. For higher aspect ratio energy harvester, reversing installation orientation reduces rotation speed as shown in Fig.79b, instead of improving it.

Numerically attained instantaneous $C_{T d}$ variation within one revolution for harvester $\mathrm{F}$ and $\mathrm{G}$ is shown in Fig.80. For harvester F, both orientations produce close $C_{\text {TdMAX }}$ values, while 'anti-clockwise' orientation is associated with less negative $\mathrm{C}_{\text {TdMIN }}$ value. Harvester $\mathrm{F}$ in 'anti-clockwise' orientation has slightly higher rotation speed than the other orientation. For harvester G, 'clockwise' orientation has less negative $\mathrm{C}_{\text {TdMIN }}$ and significantly higher $\mathrm{C}_{\text {TdMAX }}$ than 'anticlockwise' harvester G. This explains much higher rotation speed of 'clockwise' orientated harvester $\mathrm{G}$, as compared to the one in opposite orientation. 

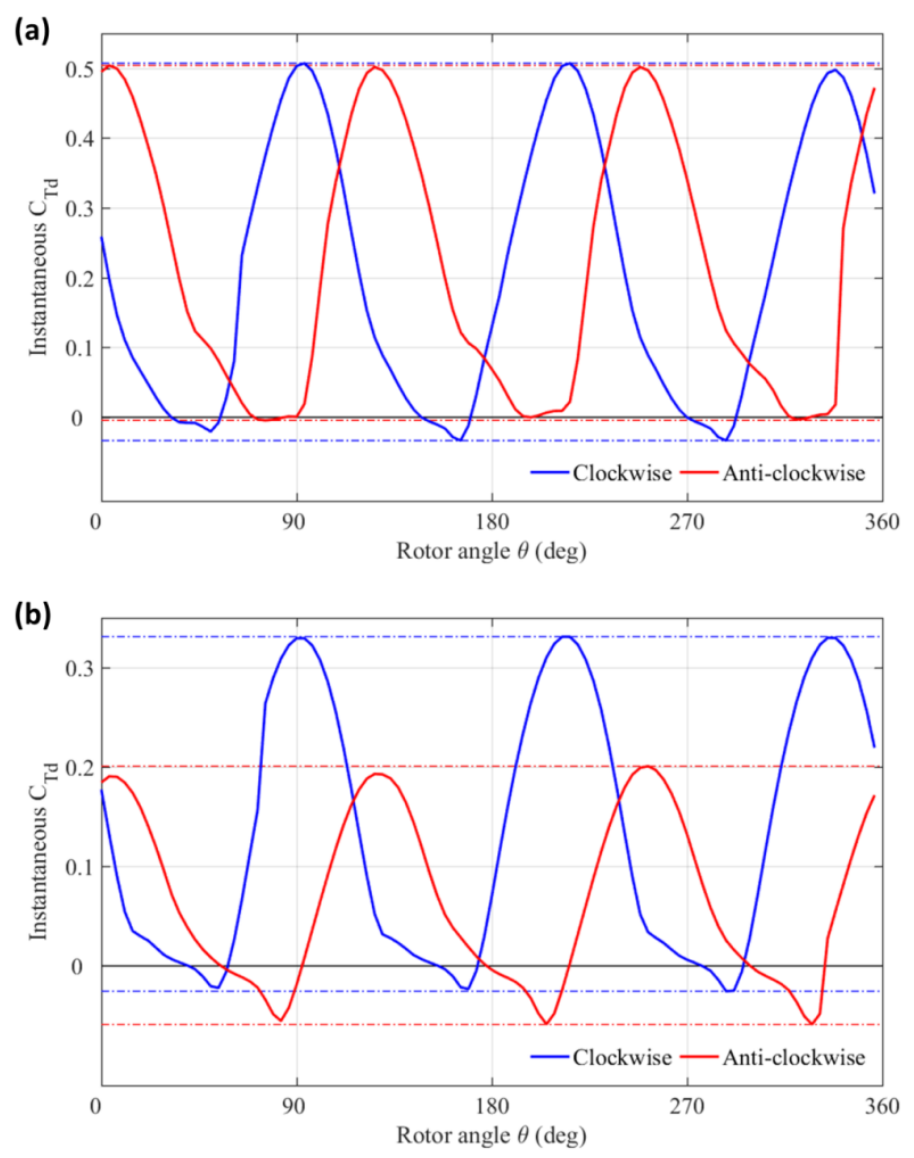

Fig.80 Instantaneous $C_{T d}$ within one revolution for energy harvester (a) $F$ and (b) $G$ at $\operatorname{Re}=1.49 \times 10^{5}$ and $\omega=5 \mathrm{rad} / \mathrm{s}$

The effect of energy harvester aspect ratio on pressure distribution around the harvester is shown in Fig.81. For harvester F, the pressure distribution shows that at $\mathrm{C}_{\mathrm{TdMAX}}$ rotor angle, the pressure differences between sides of the harvester blade for both orientations are comparable. This leads to similar $\mathrm{C}_{\mathrm{TdMAX}}$ values. At $\mathrm{C}_{\text {TdMIN }}$ rotor angles, the pressure difference on returning blade in an 'anticlockwise' orientated harvester is smaller. Therefore, the $C_{\text {TdMIN }}$ value is less negative for 'anti-clockwise' orientated harvester F. 


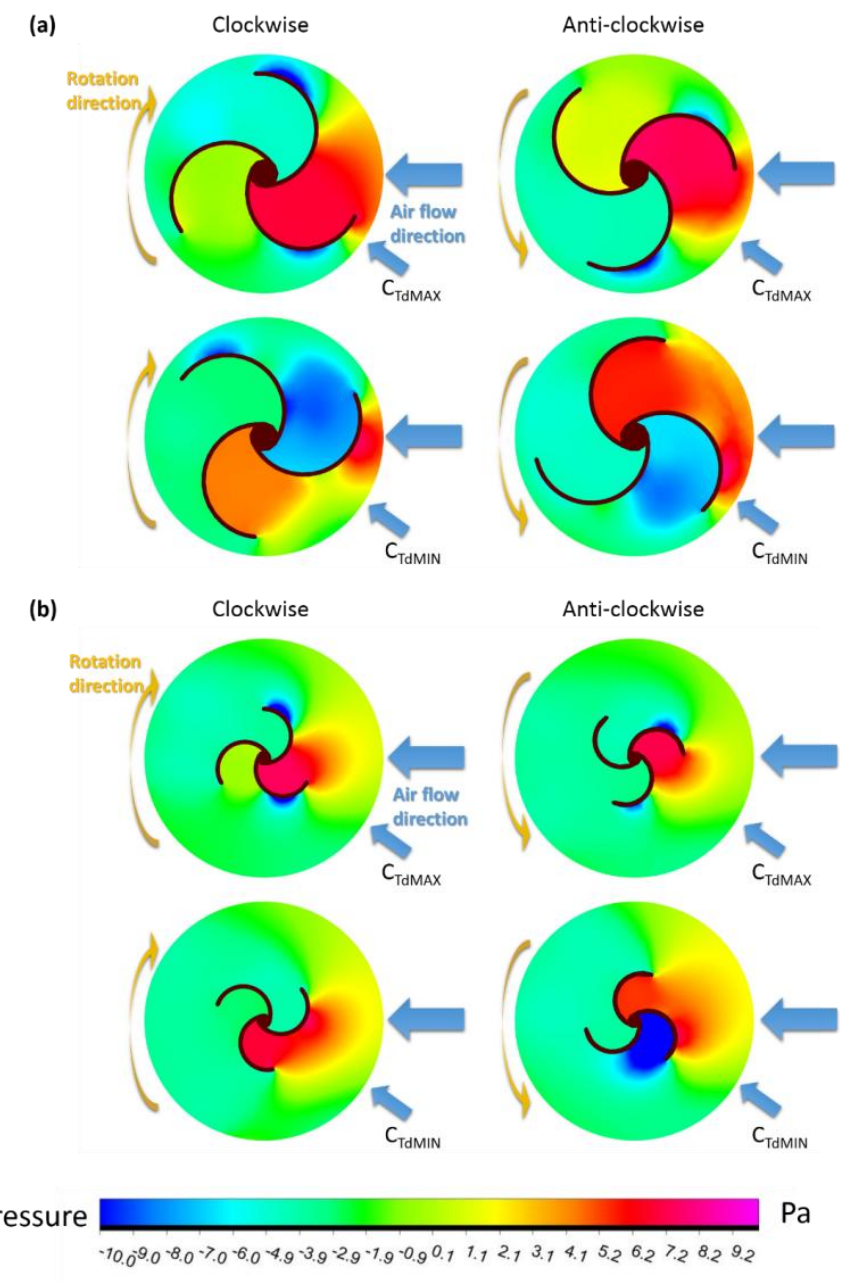

Fig.81Pressure distribution at $R e=1.49 \times 10^{5}$ for energy harvester (a) $F$ and (b) $G$ with 'clockwise' and 'anti-clockwise' installation orientation.

For harvester $\mathrm{G}$, the 'anti-clockwise' orientation produces lower $\mathrm{C}_{\text {TdMAX }}$ and more negative $\mathrm{C}_{\text {TdMIN }}$. Especially at the $\mathrm{C}_{\text {TdMIN }}$ rotor angle, the pressure difference between sides of the returning blade is much larger for 'anti-clockwise' orientation, leading to strong torque in counter-rotation direction and lower rotation speed. 


\subsubsection{Combined effect of energy harvester orientation and central part}

Effect of reversing installation orientation on harvester $\mathrm{H}$ is shown in Fig.82. As previously discussed, creation of air pathway makes the pressure less satisfactorily concentrated at the desired location neat the harvester, in both 'clockwise' and 'anti-clockwise' orientations.

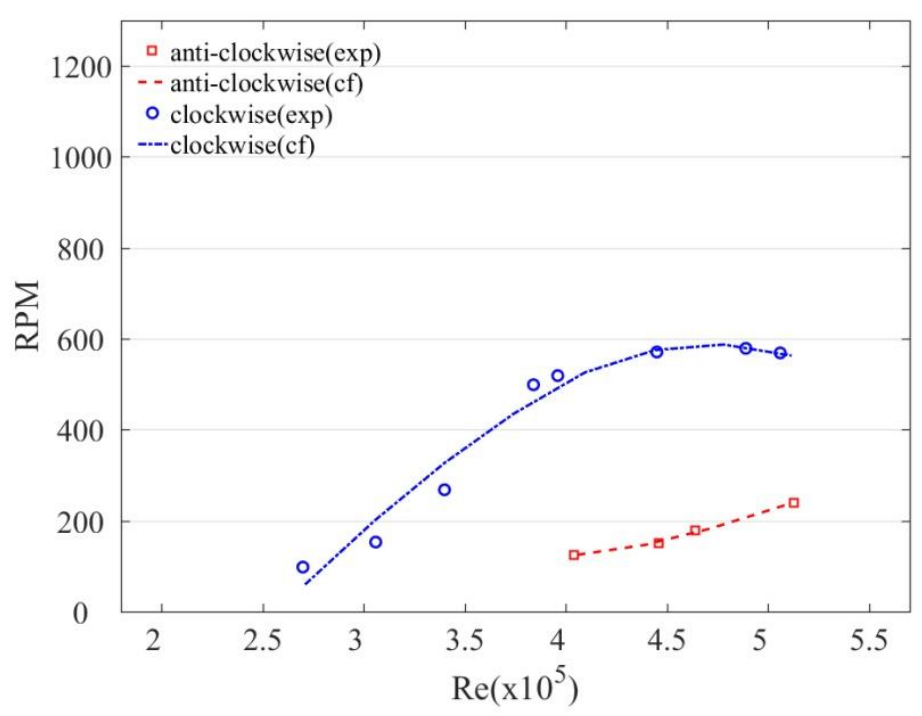

Fig.82 Variation of RPM with Re for harvester $H$ with 'clockwise' and 'anticlockwise' installation orientation. ('exp' denotes experimental data and 'cf' denotes curve fitting line)

Fig.83 is showing the numerically attained instantaneous $C_{T d}$ variation within one revolution for harvester $\mathrm{H}$ in 'clockwise' and 'anti-clockwise' orientations. It is clearly seen that 'clockwise' orientated harvester $\mathrm{H}$ is associated with higher $\mathrm{C}_{\text {TdMAX }}$ and less negative $\mathrm{C}_{\text {TdMIN }}$, as compared with harvester $\mathrm{H}$ in 'anti-clockwise' orientation. This is consistent with experimental observation that harvester $\mathrm{H}$ in 'clockwise' orientation rotates faster than it did in the state of 'anti-clockwise' orientation. 


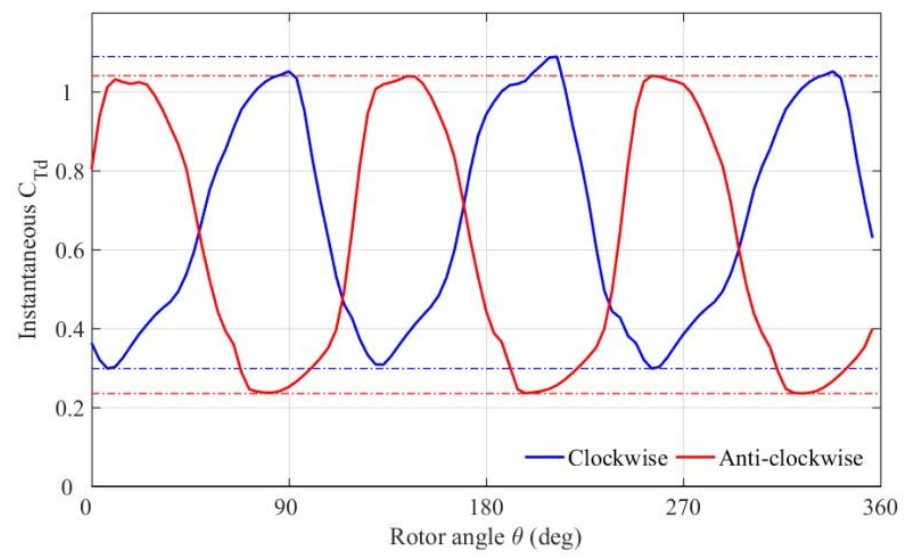

Fig.83 Instantaneous $C_{T d}$ within one revolution for energy harvester $H$ at $\operatorname{Re}=1.49 \times 10^{5}$ and $\omega=5 \mathrm{rad} / \mathrm{s}$

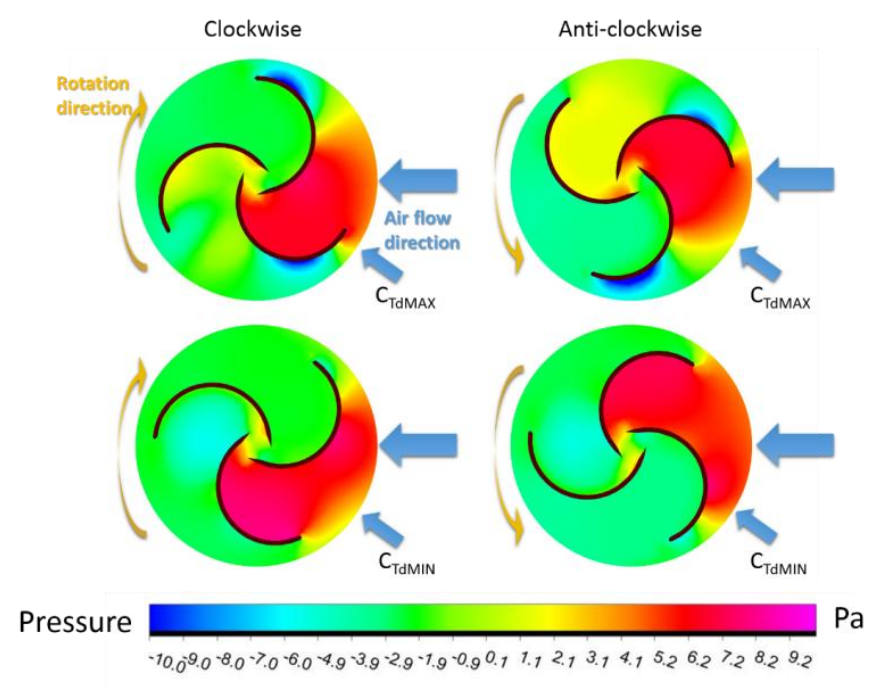

Fig.84 Pressure distribution at $R e=1.49 \times 10^{5}$ for energy harvester $H$ with 'clockwise' and 'anti-clockwise' installation orientation

The pressure distributions near energy harvester without solid central shaft in 'clockwise' and 'anti-clockwise' orientations are shown in Fig.84. As the solid central shaft is removed, air can flow via the empty gap between the blades. For both orientations, at $\mathrm{C}_{\mathrm{TdMIN}}$ rotor angles, the pressure differences between two sides of the returning blade do not differ much. However, at $\mathrm{C}_{\mathrm{TdMAX}}$ rotor angles, 
air flows through the empty central gap more easily in 'anti-clockwise' orientation, and creates smaller pressure difference between two sides of advancing blade. Hence, the driving torque in rotation direction is smaller, and the rotation speed is lower in 'anti-clockwise' orientation.

\subsubsection{Combined effect of energy harvester orientation and end plates}

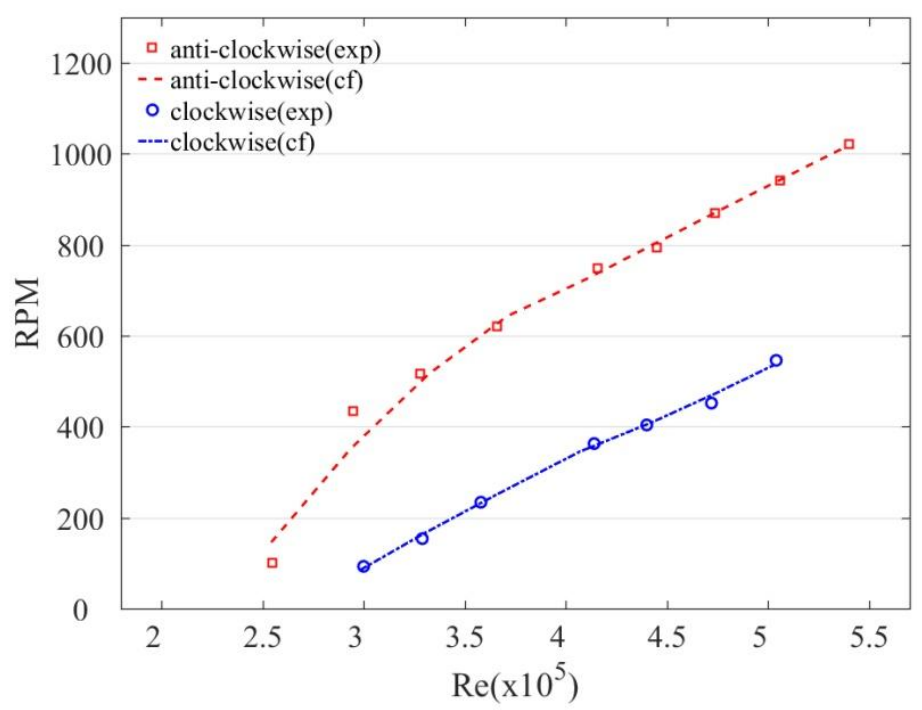

Fig.85 Variation of RPM with Re for harvester I with 'clockwise' and 'anti-clockwise' installation orientation. ('exp' denotes experimental data and 'cf' denotes curve fitting line)

Effect of reversing installation orientation on harvester $\mathrm{I}$ is shown in Fig.85. Harvester I with no end plates has pressure difference reduced by allowing air to pass via both ends of it. The pressure difference is also reduced but to a smaller extent, as pressure is still more concentrated neat the middle point of the harvester along its length direction. This allows the model to take advantage of 'anticlockwise' orientation based on the same mechanism as what happens to the 
harvester A. However, due to the fact that the pressure difference is reduced, this energy harvester possesses lower rotation speed than harvester A.

From the numerically attained instantaneous $C_{T d}$ variation within one revolution for harvester I shown in Fig.86, it is clearly seen that 'anti-clockwise' orientation is associated with much higher $\mathrm{C}_{\mathrm{TdMAX}}$. As $\mathrm{C}_{\mathrm{TdMIN}}$ values for both orientations are similar, the higher rotation speed of 'anti-clockwise' orientated harvester I was mainly contributed by higher $\mathrm{C}_{\mathrm{TdMAX}}$.

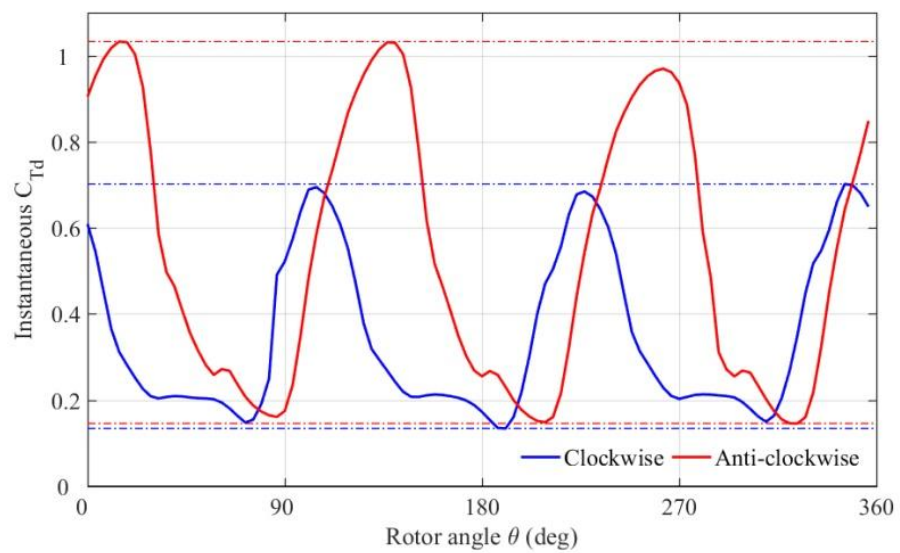

Fig.86 Instantaneous $C_{T d}$ within one revolution for energy harvester $I$ at $\mathrm{Re}=1.49 \times 10^{5}$ and $\omega=5 \mathrm{rad} / \mathrm{s}$

Fig.87 is showing pressure distribution around 'clockwise' and 'anti-clockwise' orientated harvester $\mathrm{I}$ at its $\mathrm{C}_{\mathrm{TdMAX}}$ and $\mathrm{C}_{\mathrm{TdMIN}}$ positions, respectively. It can be seen that for harvester $\mathrm{I}$ in both orientations, $\mathrm{C}_{\mathrm{TdMIN}}$ values are similar as pressure differences are similar in magnitude at $\mathrm{C}_{\text {TdMIN }}$ rotor angles. In contrast, harvester I in 'anti-clockwise' orientation can achieve higher $\mathrm{C}_{\mathrm{TdMAX}}$ value, as compared to the other orientation. The harvester in 'anti-clockwise' orientation can better capture air flows in both horizontal and upward directions, producing greater 
torque in rotation direction. Larger rotation speed is achieved. Removing end plates makes the pressure less satisfactorily focused and rotate slower than harvester A, regardless of its orientation.

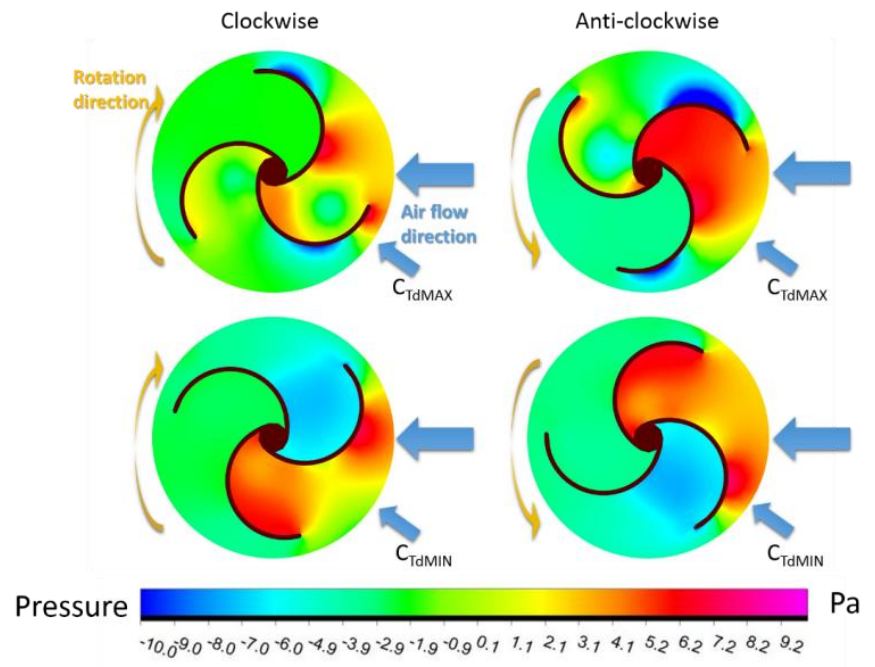

Fig.87 Pressure distribution at $R e=1.49 \times 10^{5}$ for energy harvester I with 'clockwise' and 'anti-clockwise' installation orientation

\subsubsection{Concluding remarks}

The combined effects of the harvester orientation and other design parameters are provided. in Table 10, summarizing the static performance of the harvesters in different installation orientations. It is found that altering the harvester's installation orientation does not significantly affect its static performance. A better static performance in both orientations is obtained by creating additional air pathways.

Table 10 Summary of static performance of energy harvester models in different orientations. (For each orientation, first column is experimental cut-in Reynolds number and second column is range of numerically predicted static torque coefficient.) 


\begin{tabular}{|c|c|c|c|c|}
\hline \multirow{2}{*}{ Model } & \multicolumn{2}{|c|}{ 'Clockwise' orientation } & \multicolumn{2}{|c|}{ 'Anti-clockwise' orientation } \\
\hline & $R e_{c}$ & $C_{T S}$ & $R e_{c}$ & $C_{T s}$ \\
\hline A & $\sim 5.95 \times 10^{5}$ & $-0.269 \sim 0.831$ & $\sim 8.32 \times 10^{5}$ & $-0.233 \sim 0.958$ \\
\hline B & $>9.90 \times 10^{5}$ & $-0.315 \sim 1.259$ & $>9.90 \times 10^{5}$ & $-0.020 \sim 1.047$ \\
\hline $\mathrm{C}$ & $\sim 8.89 \times 10^{5}$ & $-0.079 \sim 0.671$ & $\sim 6.58 \times 10^{5}$ & $-0.172 \sim 0.578$ \\
\hline D & $>9.90 \times 10^{5}$ & $-0.254 \sim 1.026$ & $>9.90 \times 10^{5}$ & $-0.160 \sim 0.560$ \\
\hline $\mathrm{E}$ & $>9.90 \times 10^{5}$ & $-0.100 \sim 1.135$ & $>9.90 \times 10^{5}$ & $-0.284 \sim 1.157$ \\
\hline $\mathrm{F}$ & $\sim 7.52 \times 10^{5}$ & $-0.172 \sim 0.969$ & $>9.90 \times 10^{5}$ & $-0.184 \sim 1.076$ \\
\hline G & $>9.90 \times 10^{5}$ & $-0.263 \sim 1.263$ & $>9.90 \times 10^{5}$ & $-0.152 \sim 0.454$ \\
\hline $\mathrm{H}$ & $\sim 4.33 \times 10^{5}$ & $0.339 \sim 1.053$ & $\sim 5.09 \times 10^{5}$ & $0.280 \sim 1.197$ \\
\hline I & $\sim 4.35 \times 10^{5}$ & $0.105 \sim 0.765$ & $\sim 5.84 \times 10^{5}$ & $0.064 \sim 1.253$ \\
\hline
\end{tabular}

The combined effect on the dynamic performance of the harvesters is summarized in Table 11. The experimental maximum energy conversion efficiencies of harvesters installed in different orientations are compared, and the calculated dynamic torque coefficient ranges are shown. These results confirm that, different 
from conventional Savonius wind turbine, the designed energy harvesters are orientation-sensitive. A few factors are found to affect the harvester's performance, such as whether the harvester diameter is large enough to capture the upward flow, whether the opening between the blades is wide enough for flows from both directions to enter, and so on.

Table 11Summary of dynamic performance of energy harvester models in different orientations. (For each orientation, first column is experimental overall energy conversion efficiency and second column is range of numerically predicted dynamic torque coefficient at $\omega=5 \mathrm{rad} / \mathrm{s}, R e=4.46 \times 10^{5}$.)

\begin{tabular}{|c|c|c|c|c|}
\hline \multirow{2}{*}{ Model } & \multicolumn{2}{|c|}{ 'Clockwise' orientation } & \multicolumn{2}{|c|}{ 'Anti-clockwise' orientation } \\
\hline & $\eta_{\text {overall } \max }$ & $C_{T d}$ & $\eta_{\text {overall } \max }$ & $C_{T d}$ \\
\hline A & $5.16 \%$ & $-0.0228 \sim 0.00154$ & $6.59 \%$ & $-0.0249 \sim 0.00606$ \\
\hline $\mathrm{B}$ & $2.56 \%$ & $-0.0746 \sim 2.07$ & $4.59 \%$ & $-0.0319 \sim 2.14$ \\
\hline $\mathrm{C}$ & $1.13 \%$ & $-0.0457 \sim 0.628$ & $0.27 \%$ & $-0.0796 \sim 0.532$ \\
\hline $\mathrm{D}$ & $1.86 \%$ & $-0.0357 \sim 0.287$ & $0.18 \%$ & $-0.0619 \sim 0.268$ \\
\hline $\mathrm{E}$ & $1.99 \%$ & $0.0881 \sim 1.79$ & $2.01 \%$ & $-0.134 \sim 1.88$ \\
\hline $\mathrm{F}$ & $1.13 \%$ & $-0.0334 \sim 0.507$ & $0.92 \%$ & $-0.0460 \sim 0.504$ \\
\hline $\mathrm{G}$ & $2.96 \%$ & $-0.0253 \sim 0.331$ & $0.08 \%$ & $-0.0587 \sim 0.201$ \\
\hline
\end{tabular}



$\mathrm{H}$
$1.79 \%$
$0.297 \sim 1.08$
$0.03 \%$
$0.234 \sim 1.04$
I
$0.80 \%$
$0.134 \sim 0.702$
$1.89 \%$
$0.145 \sim 1.03$

Based on the current investigation, the following observations are made:

- For three-blade energy harvesters installed at fixed location, the harvester with a larger diameter is shown to convert energy more efficiently in 'anticlockwise' orientation. And greater torque is generated, when air flows in both directions are utilized.

- For harvesters with different number of blades, the harvester with fewer blades is less effective for pressure to build up. In contrast, for the harvester with more blades, the blade might hinder air entering the central part of harvester to develop high pressure region. This blocking effect is more substantial,

- The optimal installation location of the harvester is to be determined case by case. If a step height platform is installed, then the velocity above the step height platform is varied greatly as shown in Fig. 90. The harvester should be installed in a location that airflows in both horizontal and upward directions are better utilized to generate torque in rotation direction. 


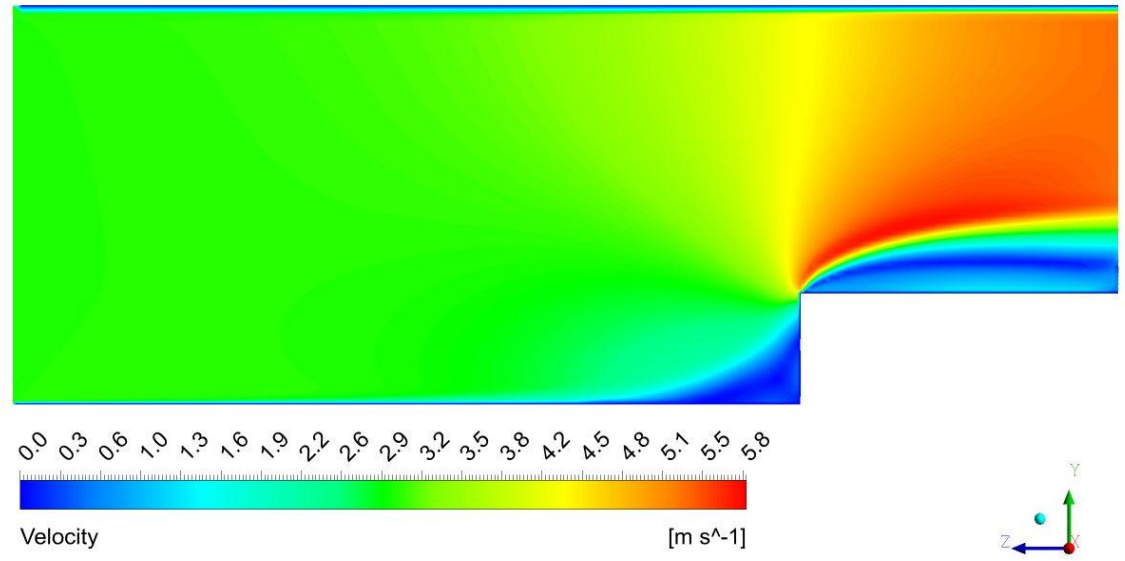

Fig.88 Velocity variation in the domain of wind tunnel test section.

\subsection{Study on blade shape profiles}

To shed light on the effect of the cross-sectional profile of harvesters' blade on its performance, further numerical simulations are conducted. Both static and dynamic performances of 8 harvesters with different cross-sectional profile shapes are evaluated and compared. The semi-cylindrical blade energy harvester described in the previous sections is chosen as a benchmark test.

The blade cross-sectional profile can be described and characterized by establishing a coordinate system, where the origin is the center part of the harvester where the blades are joined. The span of $\mathrm{X}$ coordinate is from $0 \%$ to $100 \%$, and it corresponds to the chord length of the blade. The span of Y coordinate is from $0 \%$ to $50 \%$, which has the same length scale as $\mathrm{X}$ coordinate, and it corresponds to the distance above the chord in the direction perpendicular to the chord. The blade 
cross-sectional profiles are mainly characterized by the different placements of the highest point.
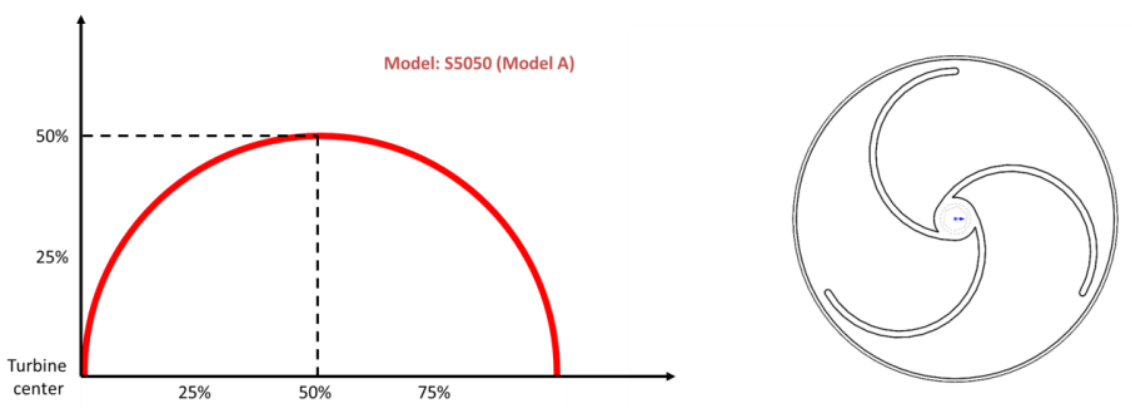

Fig.89 Blade cross-sectional profile design of S5050 model (harvester J).

The first blade cross-sectional profile is the semi-cylindrical blade, and it is the benchmark model in 'anti-clockwise' orientation (harvester J). It has a 50\% camber value placed at $50 \%$ of the chord line. Thus it is denoted as S5050 (Fig.89), where the letter ' $S$ ' indicates there is a single highest point, the first two digits indicate the placement of the highest point along the chord length of the blade and the last two digits indicate the location of the highest point above the chord of the blade. The other harvesters are designed by varying highest point(s) location. The other seven designs are S2550 (Fig.92), S7550 (Fig.95), S5025 (Fig.98), S2525 (Fig.101), S7525 (Fig.104), D2550-7550 (Fig.107) and D2525-7525 (Fig.110). For the last two designs, the letter ' $\mathrm{D}$ ' indicates there are two highest points, and the two groups of digits indicate the locations of these two highest points, respectively.

The performance of S5050 model (harvester J) has already been presented in details hence is not repeated in details here. Its static torque coefficient distribution at different rotor angles can be found in Fig.90, and its power coefficients at 
different tip speed ratios can be found in Fig.91.The numerical study results on static and dynamic performances of the energy harvesters with other cross-section profile shapes are presented in the following sections. The static torque coefficients for all models are determined at $\operatorname{Re}=1.98 \times 10^{5}$. The power coefficients are obtained at $\operatorname{Re}=1.49 \times 10^{5}$. In both static and dynamic studies, the energy harvester models are all set in 'anti-clockwise' orientation. For harvester model J, the static torque performance has minimum value of -0.234 , and maximum value of 0.98. The maximum power coefficient attained is 0.193 at $\lambda=0.833$.

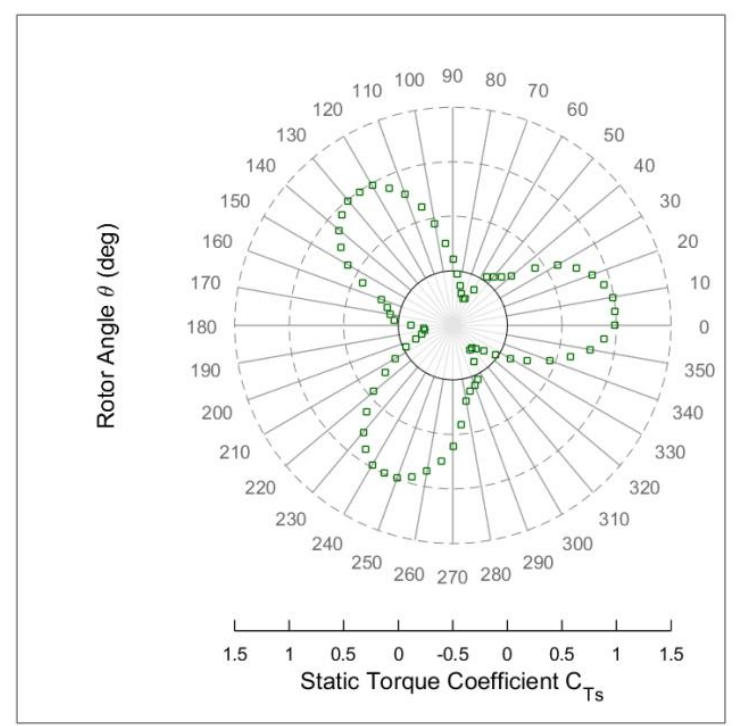

Fig.90 Variation of static torque coefficients at different rotor angles at $\mathrm{Re}=\mathbf{1 . 9 8} \times 10^{5}$ forenergy harvester $\mathrm{J}$ 


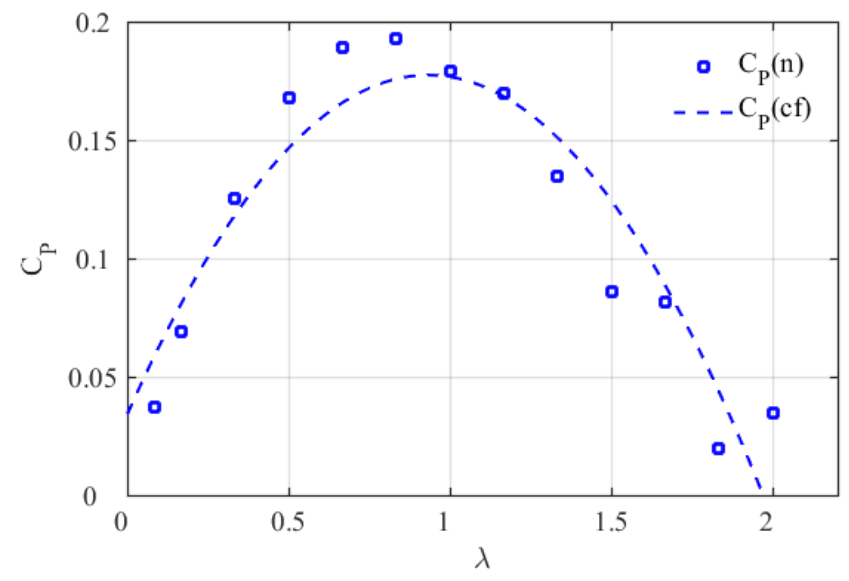

Fig.91 Performance characteristic curves for energy harvester $\mathrm{J}$, at $\mathrm{Re}=1.49 \times 10^{5}$. (c-f denotes curve fitting and $n$ denotes numerical results).

\subsubsection{Energy harvester with cross-sectional profile shape $\mathbf{S 2 5 5 0}$}
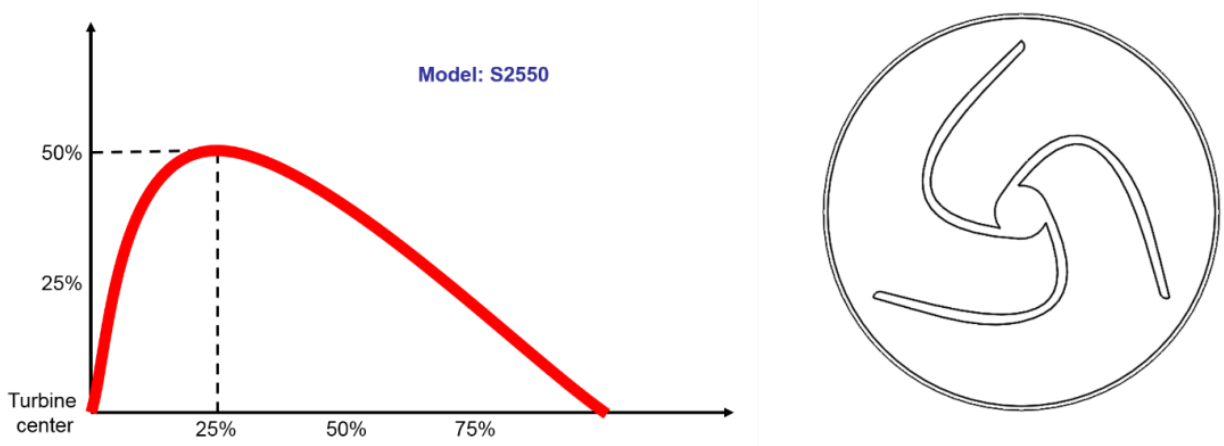

Fig.92 Blade cross-sectional profile design of S2550 model

Fig.92 is showing the cross-sectional profile with S2550 shape. The highest point above the chord is at the same height as that of harvester $\mathrm{J}$, but closer to the harvester center.

The variation of static torque coefficient distribution at different rotor angles for this model is shown in Fig.93. It can be seen that both positive and negative $C_{T s}$ exist: $C_{T s}$ is negative in the rotor angle ranges of $65^{\circ}-90^{\circ}, 185^{\circ}-210^{\circ}$ and $305^{\circ}-330^{\circ}$, 
indicating the model has difficulty in self-starting rotation in these ranges. The maximum $C_{T S}$ take place at rotor angles of $5^{\circ}, 125^{\circ}$ and $245^{\circ}$, while the minimum $C_{T s}$ occurs at $80^{\circ}, 200^{\circ}$ and $320^{\circ}$. The maximum and minimum $C_{T s}$ rotor angles give the most and least favorable positions for the model to self-start rotation, respectively. As compared to harvester J, S2550 model has maximum $C_{T s}$ reduced to 0.68 from 0.98 and more negative minimum $C_{T s}$ of -0.324 , indicating a worse self-starting capability than harvester J.

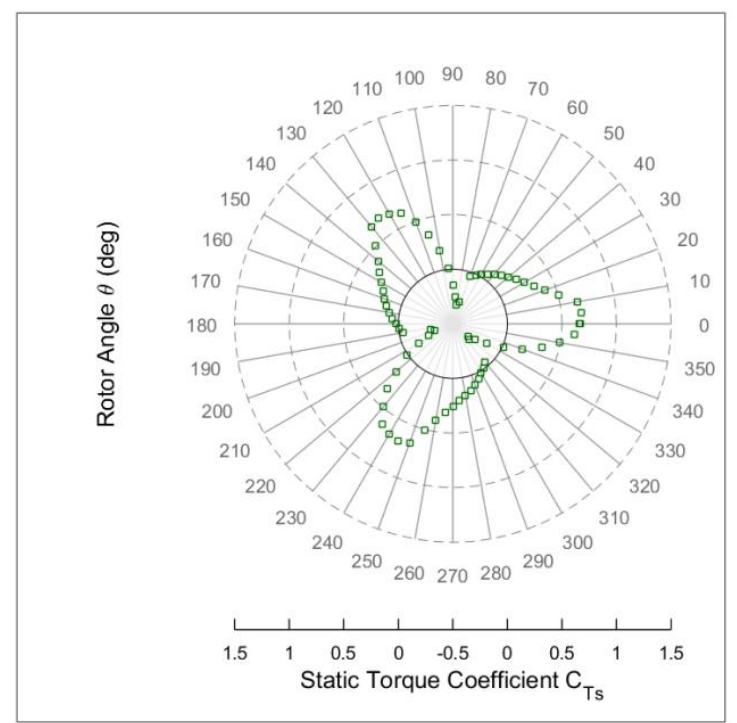

Fig.93 Variation of static torque coefficients at different rotor angles at $\operatorname{Re}=1.98 \times 10^{5}$ for energy harvester with $\mathbf{S 2 5 5 0}$ cross sectional profile shape.

The dynamic performance of S2550 model can be numerically studied from its power coefficient, as shown in Fig.94. The maximum power coefficient for this model is $C_{P \max } \approx 0.15$. The lower maximum power coefficient indicates this S2550 harvester model is less efficient in conversion of energy, as compared to harvester $\mathrm{J}$. 


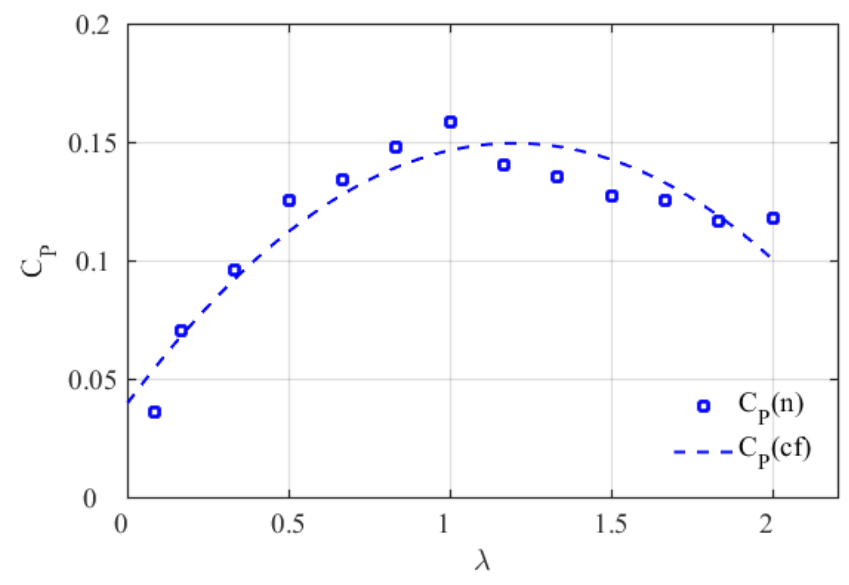

Fig.94Performance characteristic curves for energy harvester with S2550 cross sectional profile shape, at $R e=1.49 \times 10^{5}$. (c-f denotes curve fitting and $n$ denotes numerical results).

Based on these numerical study results, harvester model with S2550 cross-sectional profile shape performs worse than harvester $\mathrm{J}$, in terms of both static and dynamic perspectives.

\subsubsection{Energy harvester with cross-sectional profile shape $S 7550$}
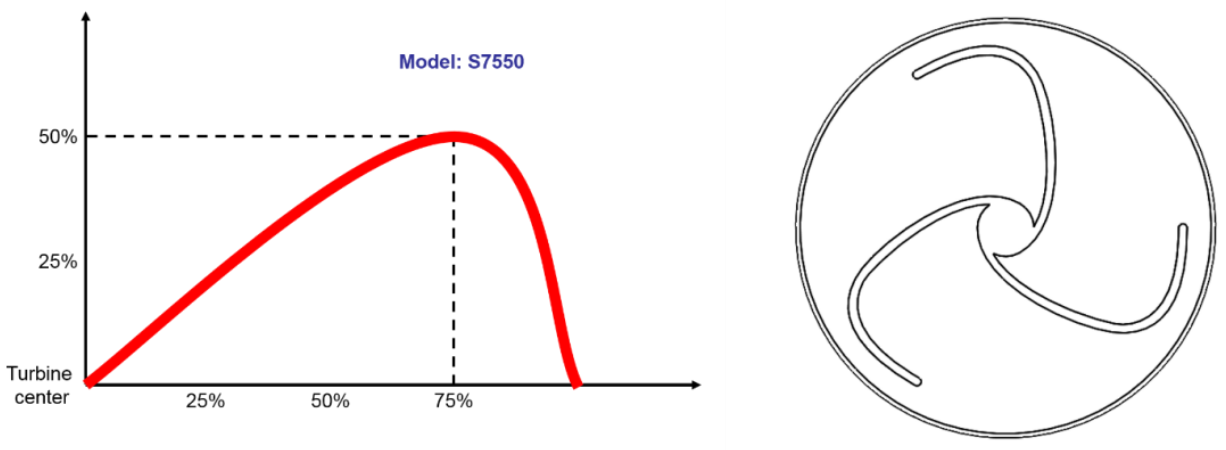

Fig.95 Blade cross-sectional profile design of S7550 model 
The cross-sectional profile with S7550 shape is shown in Fig.95, and it can be seen that compared to harvester $\mathrm{J}$, the highest point is at the same distance from the chord but further from harvester central point.

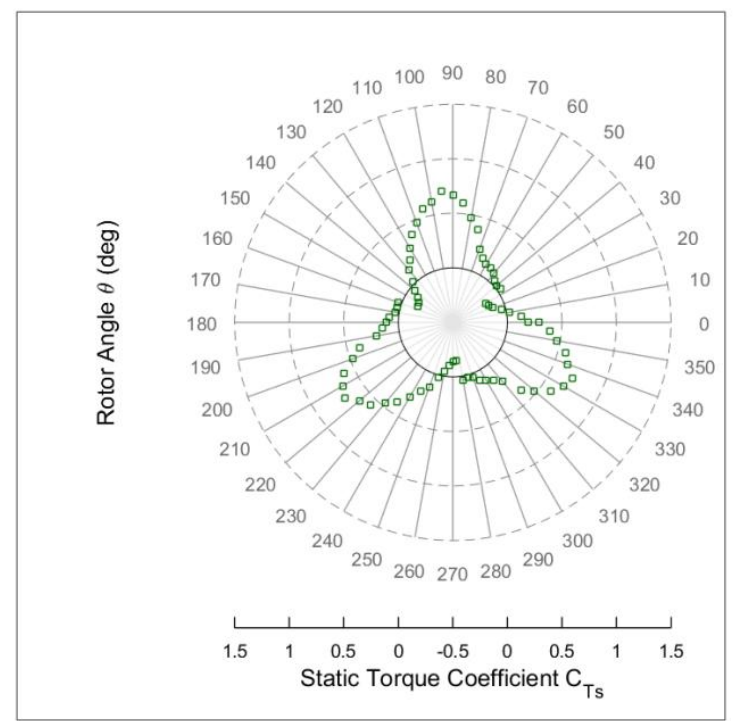

Fig.96 Variation of static torque coefficients at different rotor angles at $\mathrm{Re}=1.98 \times 10^{5}$ for energy harvester with $\mathbf{S 7 5 5 0}$ cross sectional profile shape.

The static torque coefficient distribution at different rotor angles is shown in Fig.96. Both positive and negative static torque coefficients exist. Undesirable starting positions are in rotor angle ranges of $20^{\circ}-35^{\circ}, 140^{\circ}-155^{\circ}$ and $260^{\circ}-275^{\circ}$, as the static torque coefficients are negative in these ranges. The maximum values of static torque coefficient take place at $95^{\circ}, 215^{\circ}$ and $335^{\circ}$, but the values are smaller than that of harvester J. The minimum static torque coefficient values are less negative than that of harvester $\mathrm{J}$ and occur at $35^{\circ}, 155^{\circ}$ and $275^{\circ}$. The maximum value is 0.707 and the minimum value is -0.148 . As compared to harvester $\mathrm{J}$, maximum 
static torque coefficient is lower, but minimum value is less negative, it is difficult to predict whether $\mathrm{S} 7550$ model has improved static performance.

Fig.97 is showing power coefficients of S7550 harvester model at different tip speed ratios. It can be clearly seen that the theoretical maximum power coefficient of 0.186 is close that of harvester J. However, it must be pointed out that the high power coefficient of S7550 model take place at high tip speed ratios $(\lambda=2.0)$, which are very difficult to be practically achieved. Hence, the model is unlikely to convert energy more efficiently than harvester $\mathrm{J}$.

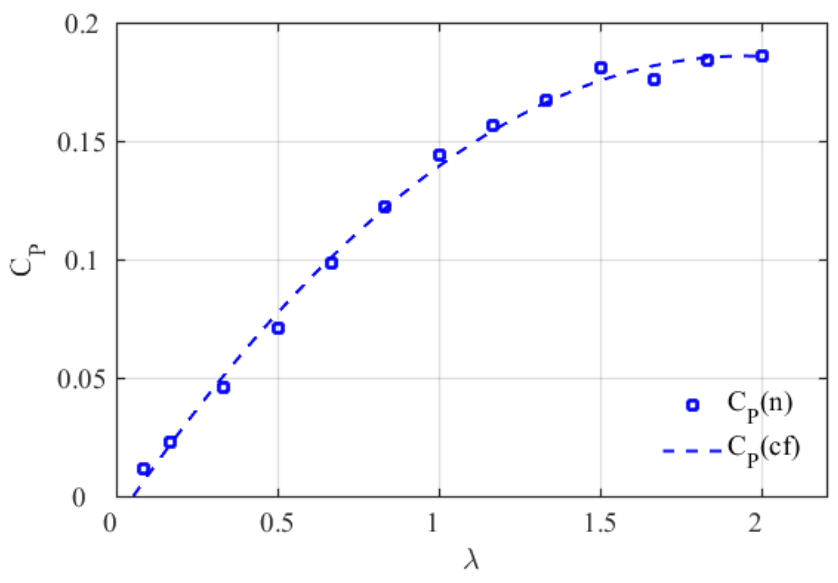

Fig.97 Performance characteristic curves for energy harvester with S7550 cross sectional profile shape, at $\operatorname{Re}=1.49 \times 10^{5}$. (c-f denotes curve fitting and $n$ denotes numerical results).

Based on numerical results attained for model with S7550 cross-sectional profile shape, it is difficult to make judgments about its static performance. However, for its dynamic performance, it is unlikely to be more efficient than harvester J. 


\subsubsection{Energy harvester with cross-sectional profile shape $\mathbf{S 5 0 2 5}$}
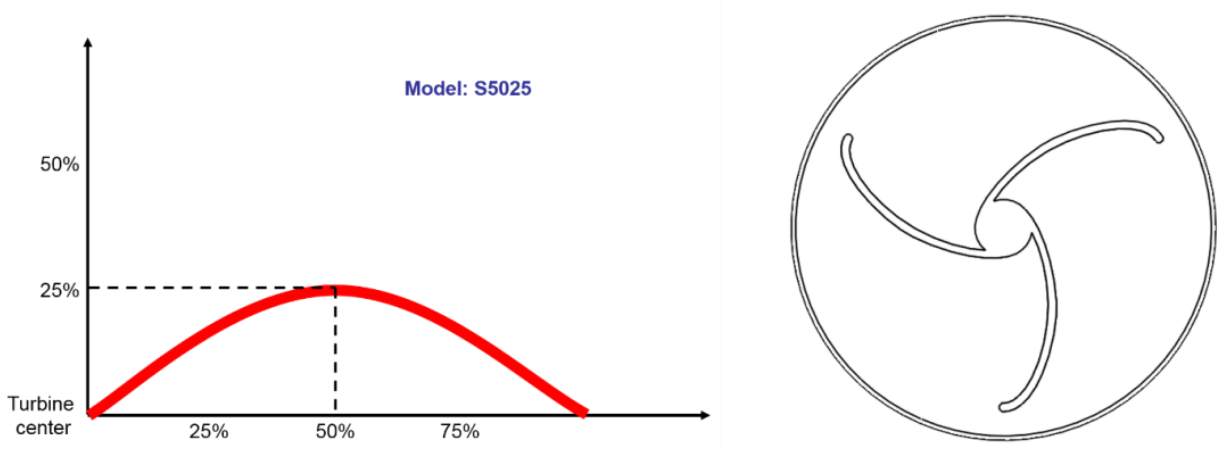

Fig.98 Blade cross-sectional profile design of S5025 model

Fig.98 is showing the cross-sectional profile with S5025 shape. Its highest point is at the middle of the chord, but the distance to the chord is smaller.

The static performance of this model can be numerically studied from Fig.99. According to static torque coefficient distribution graph, it can be seen that positive and negative coefficients both exist. Negative static torque coefficient values can be found in the rotor angle ranges of $70^{\circ}-85^{\circ}, 190^{\circ}-205^{\circ}$ and $310^{\circ}-325^{\circ}$. The maximum static torque coefficient is higher than that of harvester $\mathrm{J}$, and can be found at $5^{\circ}, 125^{\circ}$ and $245^{\circ}$. The minimum static torque coefficient value is close to that of harvester $\mathrm{J}$, and takes place at $80^{\circ}, 200^{\circ}$ and $320^{\circ}$. Its static torque coefficient has maximum value of 1.145 and minimum value of -0.251 . Due to higher maximum static torque coefficient value and similar minimum static torque coefficient, S5025 model is expected to self-start rotation at lower Reynolds number, as compared to harvester $\mathrm{J}$. 


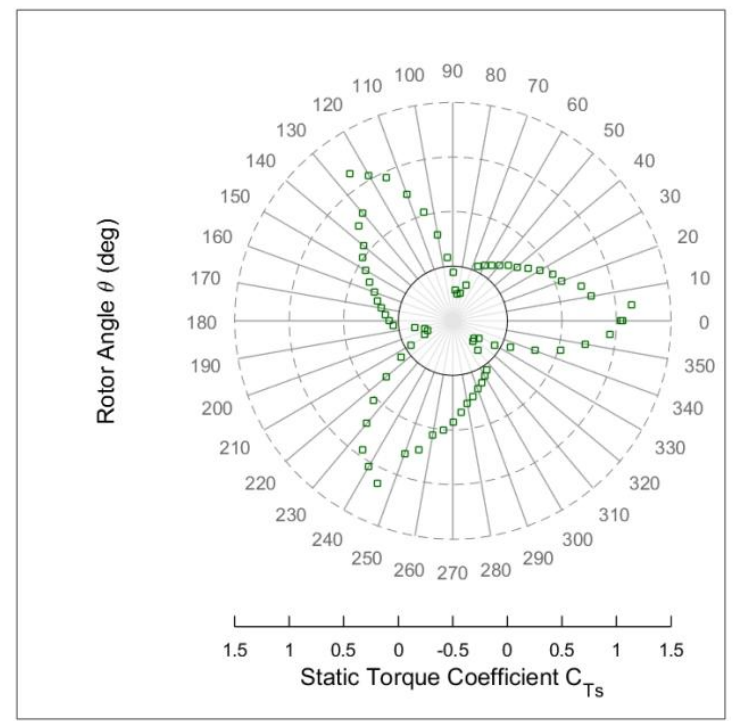

Fig.99Variation of static torque coefficients at different rotor angles at $\mathrm{Re}=1.98 \times 10^{5}$ for energy harvester with $\mathbf{S 5 0 2 5}$ cross sectional profile shape.

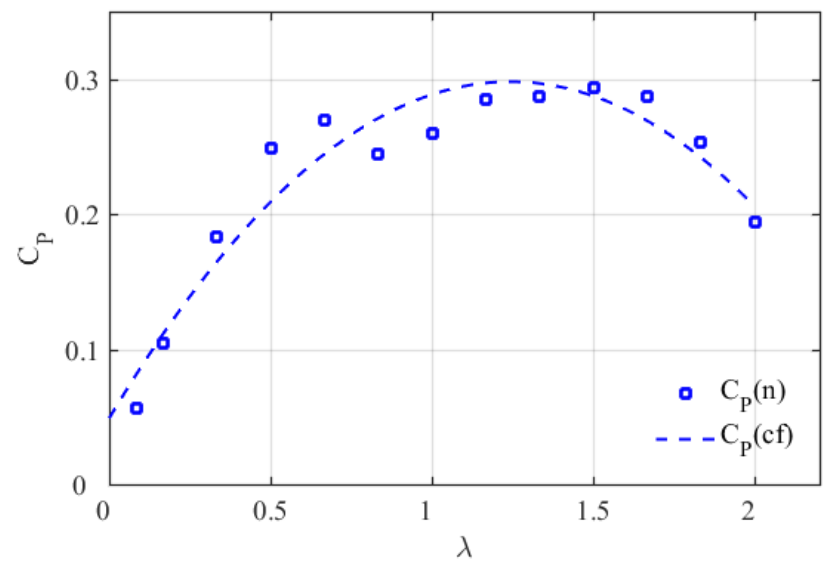

Fig.100Performance characteristic curves for energy harvester with 55025 cross sectional profile shape, at $\mathrm{Re}=1.49 \times 10^{5}$. (c-f denotes curve fitting and $\mathrm{n}$ denotes numerical results).

The power coefficient at different values of tip speed ratios for S5025 model can be seen in Fig.100. The power coefficient is higher than that of harvester $\mathbf{J}$ at all range of tip speed ratios under investigation, and the highest power coefficient attained is 
0.294. Therefore, it is expected for S5025 model to harness energy more efficiently than harvester J.

Based on numerical results attained, the energy harvester model with S5025 crosssectional profile shape is expected to perform better than harvester $\mathrm{J}$, both in terms of static and dynamic performance perspectives.

\subsubsection{Energy harvester with cross-sectional profile shape $\mathbf{S 2 5 2 5}$}
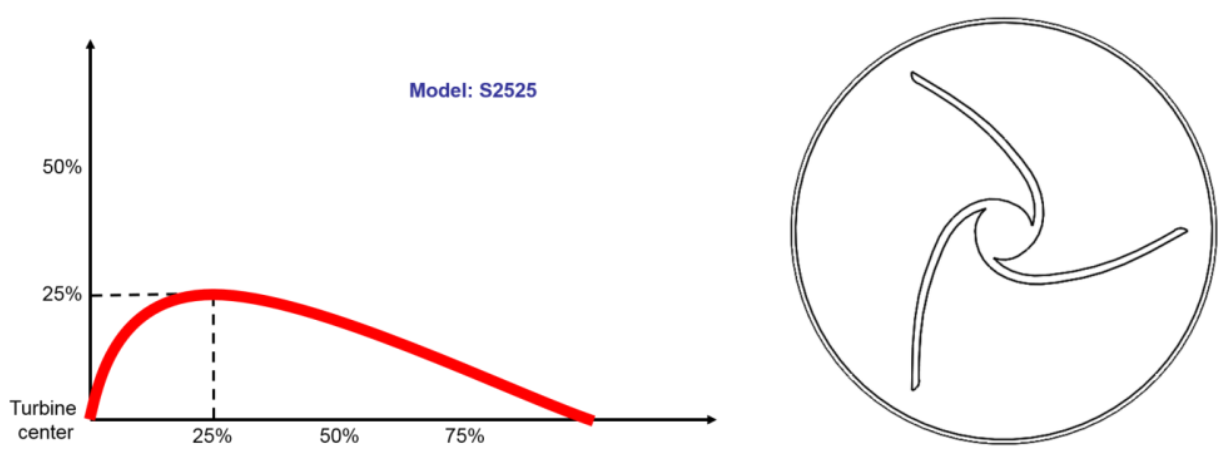

\section{Fig.101 Blade cross-sectional profile design of S2525 model}

Fig.101 is showing the energy harvester model with S2525 cross-sectional profile shape. Its highest point is both closer to the chord and the harvester center point.

The static performance of S2525 model can be studied from Fig.102. It can be seen that both positive and negative static torque coefficients exist, however, the ranges of negative static torque coefficient are wider than that of harvester J. For S2525 harvester, the static torque coefficients are found to be negative in rotor angle ranges of $5^{\circ}-50^{\circ}, 125^{\circ}-170^{\circ}$ and $245^{\circ}-290^{\circ}$. The maximum static torque coefficient value of 0.871 is found at $95^{\circ}, 215^{\circ}$ and $335^{\circ}$, and the value is lower than that of 
harvester J. The minimum static torque coefficients are at $40^{\circ}, 160^{\circ}$ and $280^{\circ}$, and it is -0.238 and is close that of harvester $\mathrm{J}$. Due to the wider range of negative static torque coefficient, the S2525 model is expected to have larger cut-in Reynolds number than harvester $\mathrm{J}$.

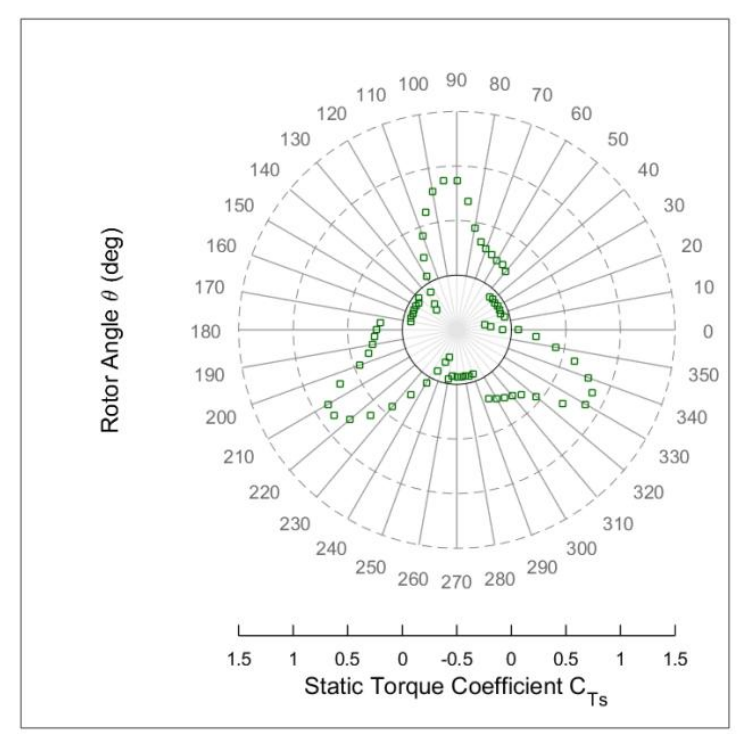

Fig.102 Variation of static torque coefficients at different rotor angles at $\operatorname{Re}=1.98 \times 10^{5}$ for energy harvester with $\mathbf{S 2 5 2 5}$ cross sectional profile shape.

The power coefficients at different tip speed ratios for S2525 model are plotted in Fig.103. It can be seen that S2525 model has potential to achieve higher power coefficient of 0.232 , as compared to harvester J. However, this requires the harvester model to be operating at high tip speed ratio of 2.0 , which is very practically challenging. Hence, it may not be operating more efficiently than harvester $\mathrm{J}$ in real life. 


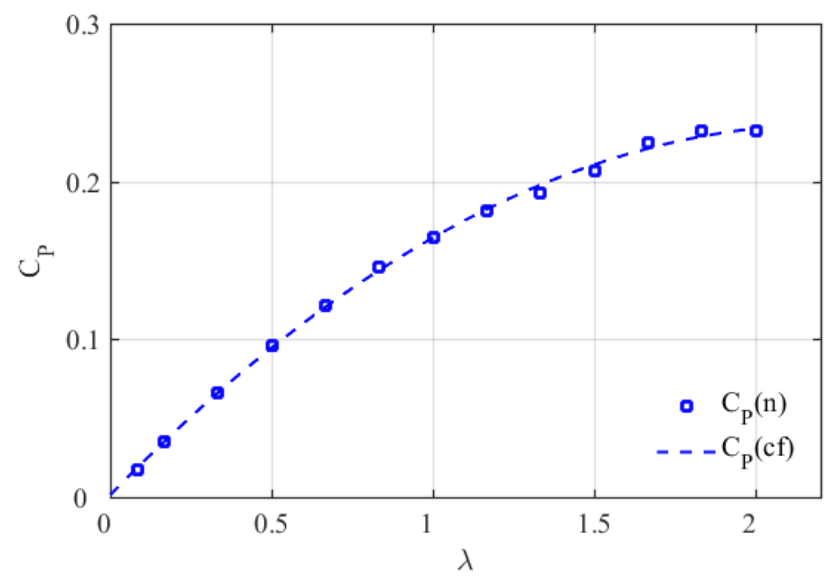

Fig.103Performance characteristic curves for energy harvester with S2525 cross sectional profile shape, at $R e=1.49 \times 10^{5}$. (c-f denotes curve fitting and $n$ denotes numerical results).

The numerical results showed that the S2525 model does not perform as well as harvester $\mathbf{J}$ in terms of static performance, as it exhibits wide ranges of negative static torque coefficients. S2525 model has potential to achieve high energy conversion efficiency at high tip speed ratios, however, at practically achievable lower tip speed ratios it is less efficient than harvester $\mathrm{J}$.

\subsubsection{Energy harvester with cross-sectional profile shape $S 7525$}
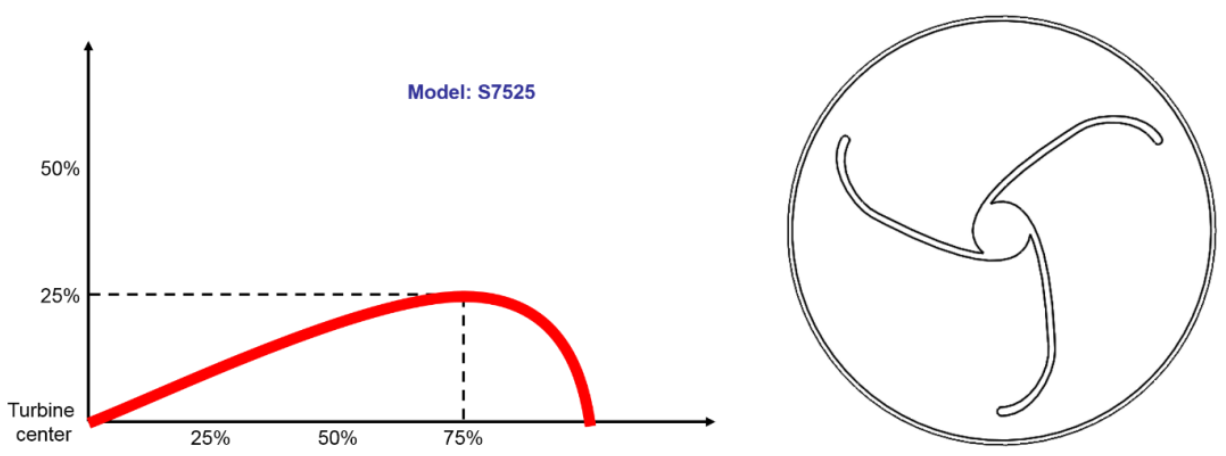

Fig.104 Blade cross-sectional profile design of S7525 model 
The design of energy harvester model with S7525 cross-sectional profile shape is shown in Fig.104. Its highest point is located nearer to the chord, but further away from the harvester center point, as compared to harvester J.

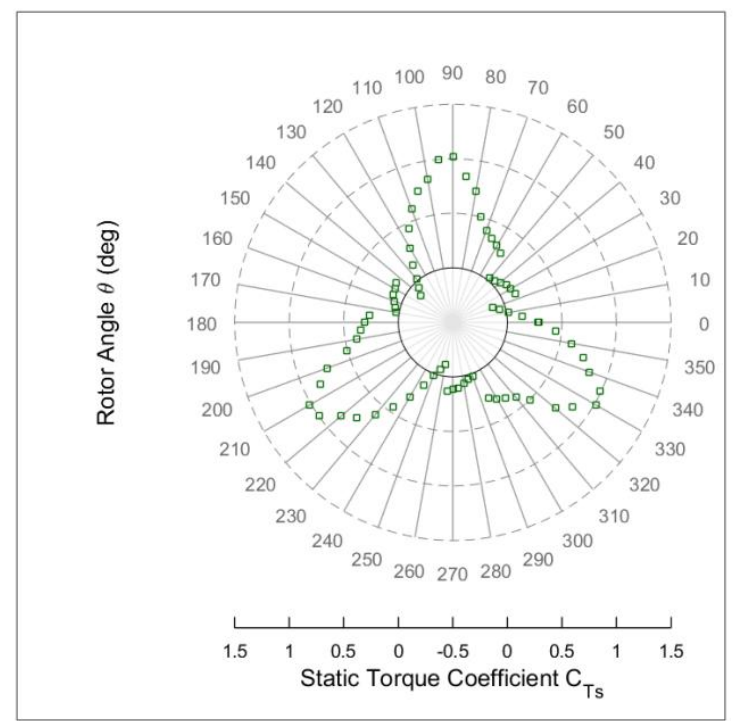

Fig.105 Variation of static torque coefficients at different rotor angles at $\mathrm{Re}=1.98 \times 10^{5}$ for energy harvester with $\mathbf{S 7 5 2 5}$ cross sectional profile shape.

Static torque coefficient distribution at different rotor angles is shown in Fig.105. Although both positive and negative static torque coefficients both exist, the ranges for negative static torque coefficient are very narrow. The ranges of negative torque coefficient are for rotor angles of $15^{\circ}-20^{\circ}, 135^{\circ}-140^{\circ}$ and $255^{\circ}-260^{\circ}$. Additionally, the maximum static torque coefficient of 1.02 is larger than that of harvester $\mathrm{J}$, and the minimum static torque coefficient of -0.112 is less negative than that of harvester J. For S7525 model, the maximum static torque coefficient take place at $90^{\circ}, 210^{\circ}$ and $330^{\circ}$, while the minimum static torque coefficient occurs at $20^{\circ}$, 
$140^{\circ}$ and $260^{\circ}$. All of these results suggest that the S7525 model is expected to selfstart rotation at lower cut-in Reynolds number, as compared to harvester J.

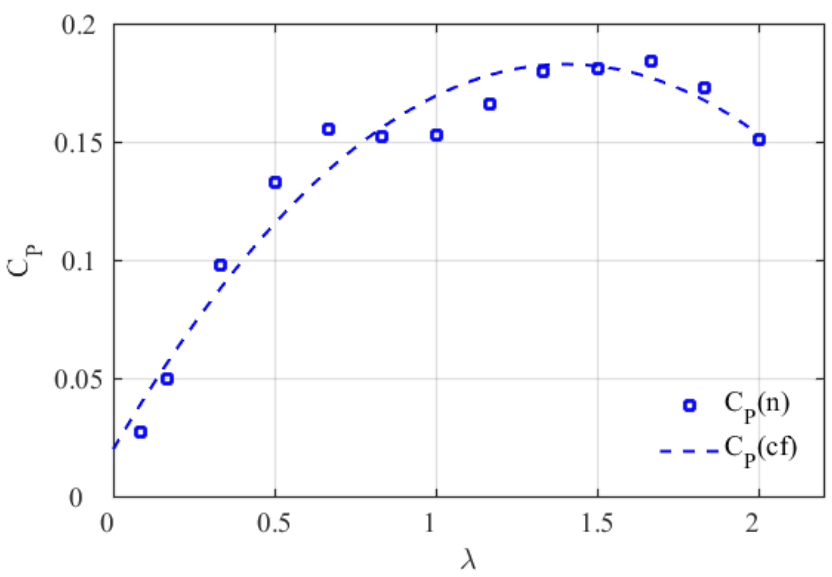

Fig.106 Performance characteristic curves for energy harvester with S7525 cross sectional profile shape, at $R e=1.49 \times 10^{5}$. (c-f denotes curve fitting and $n$ denotes numerical results).

The variation of power coefficient with tip speed ratio for S7525 model is shown in Fig.106. It can be seen that S7525 model has potential to achieve similar power coefficient of 0.166 to that of harvester $\mathrm{J}$, but a higher tip speed ratio is necessary for this to be realized. Therefore, S7525 model is expected to convert energy less efficiently than harvester $\mathrm{J}$.

The attained numerical results show that $\mathrm{S} 7525$ model is expected to exhibit better self-starting capability than harvester J. Additionally, the energy conversion efficiency of S7525 is expected to be lower than that of harvester J. 


\subsubsection{Energy harvester with cross-sectional profile shape D2550-7550}
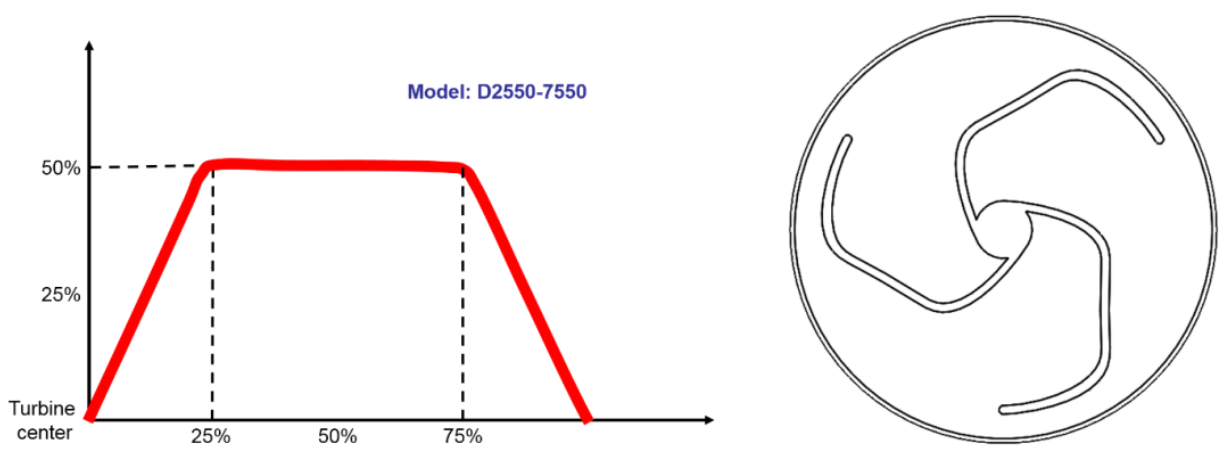

Fig.107 Blade cross-sectional profile design of D2550-7550 model

Fig.107 is showing the energy harvester model with D2550-7550 cross-sectional profile shape. Instead of having one single highest point as the models previously described, this model has two highest points and both of them are located the same distance away from the chord as the case for harvester $\mathbf{J}$.

The static torque coefficient distribution at different rotor angles can be found in Fig.108. Both positive and negative static torque coefficients exist, and negative values could be found in rotor angle ranges of $50^{\circ}-85^{\circ}, 170^{\circ}-205^{\circ}$ and $290^{\circ}-325^{\circ}$. The maximum static torque coefficient value of 0.657 is less than that of harvester $\mathrm{J}$, and it happens at rotor angles of $0^{\circ}, 120^{\circ}$ and $240^{\circ}$. Its minimum static torque coefficient of -0.192 is about the same in magnitude as that of harvester $\mathrm{J}$, and it can be found at rotor angles of $65^{\circ}, 185^{\circ}$, and $305^{\circ}$. Based on these findings, the D2550-7550 model is expected to have less desirable self-starting capability as compared to harvester $\mathrm{J}$. 


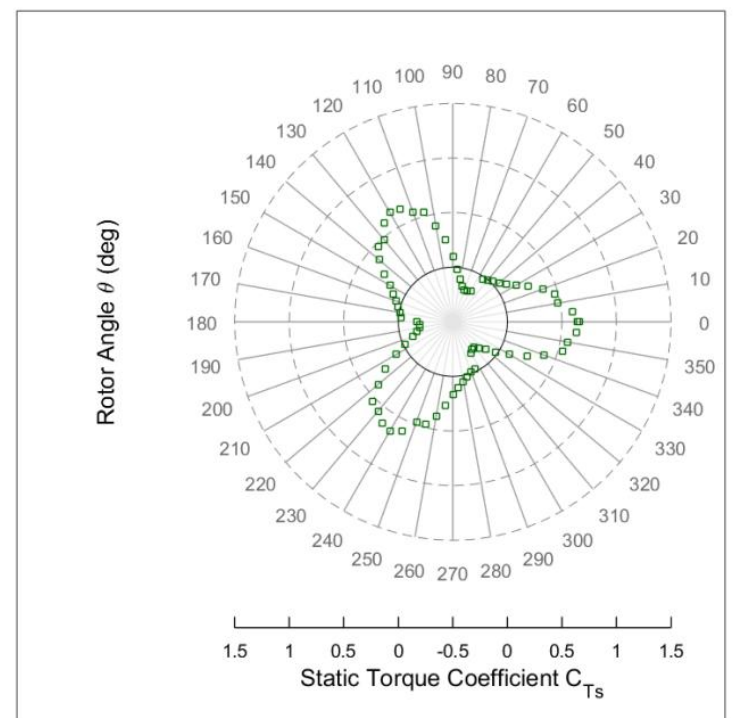

Fig.108 Variation of static torque coefficients at different rotor angles at $\operatorname{Re}=1.98 \times 10^{5}$ for energy harvester with D2550-7550 cross sectional profile shape.

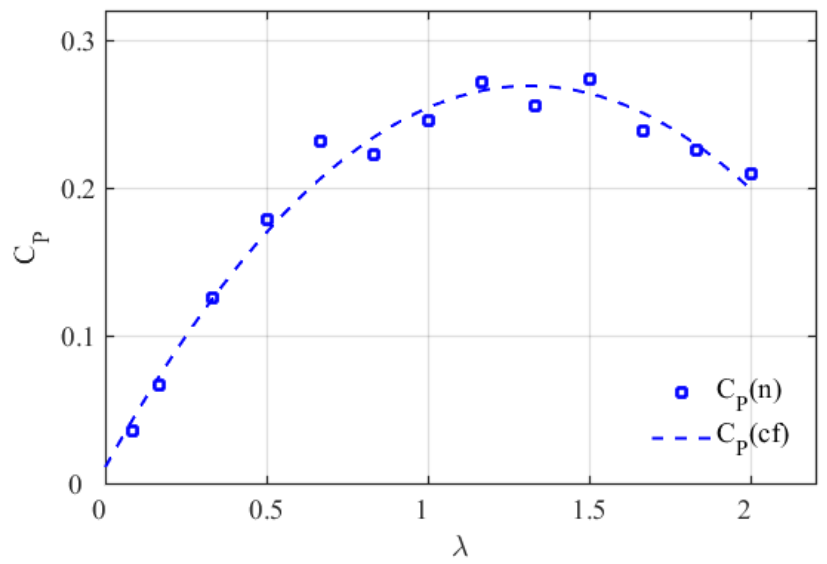

Fig.109 Performance characteristic curves for energy harvester with D2550-7550 cross sectional profile shape, at $\mathrm{Re}=1.49 \times 10^{5}$. (c-f denotes curve fitting and $\mathrm{n}$ denotes numerical results).

The power coefficient variation with different tip speed ratios for D2550-7550 model can be found in Fig.109. Higher power coefficient is found for this model at all tip speed ratios, as compared to harvester J. The highest power coefficient can 
be achieved is 0.272 . Hence, it is expected to harness energy more efficiently than harvester J.

The above results show that D2550-7550 model is expected to harness energy more efficiently than harvester J, however the cut-in Reynolds number will be higher.

\subsubsection{Energy harvester with cross-sectional profile shape D2525-7525}
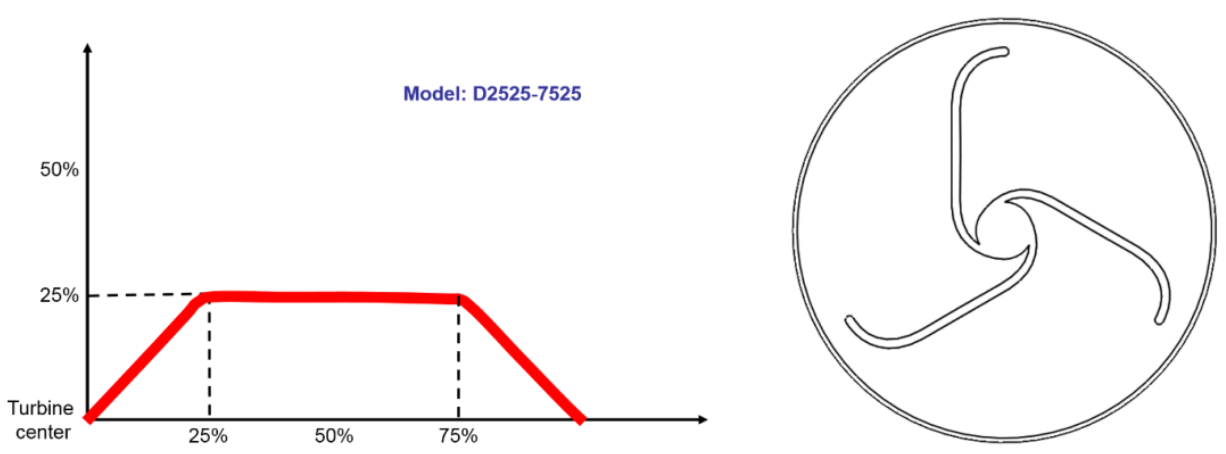

Fig.110 Blade cross-sectional profile design of D2525-7525 model

Fig.110 is showing the design of D2525-7525 model. Same as the previous model, it also has two highest points, but the two points have smaller distance away from the chord.

The static torque coefficient for this model can be found in Fig.111. Both positive and negative static torque coefficients exist, and negative values could be found in rotor angle ranges of $15^{\circ}-30^{\circ}, 135^{\circ}-150^{\circ}$ and $255^{\circ}-270^{\circ}$. The maximum static torque coefficient value of 0.916 is close to that of harvester $\mathrm{J}$, and can be found at rotor angles of $90^{\circ}, 210^{\circ}$, and $330^{\circ}$. The minimum static torque coefficient of -0.125 is slightly higher than that of harvester $\mathrm{J}$, and can be found at $25^{\circ}, 145^{\circ}$ and $265^{\circ}$. 
Based on these findings, it is expected for D2525-7525 model to exhibit similar cut-in Reynolds number.

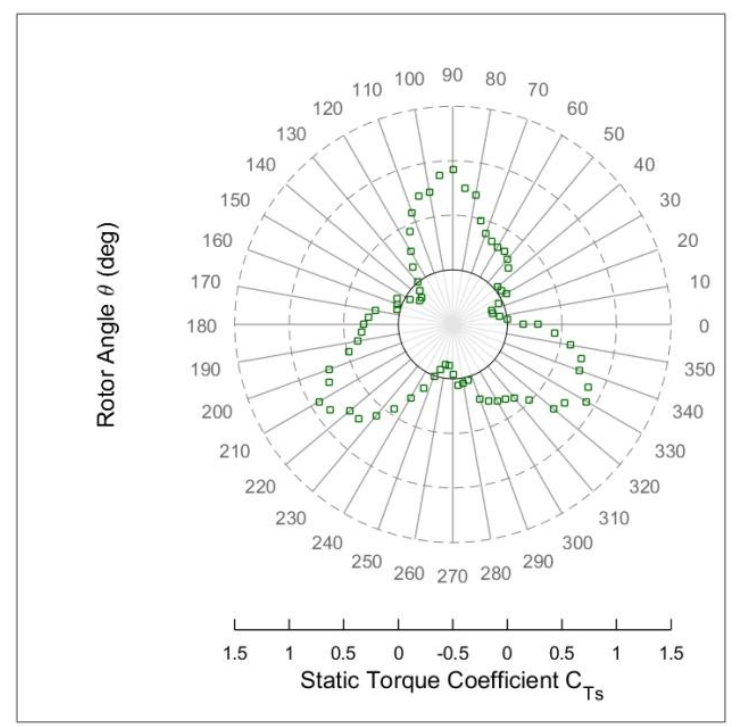

Fig.111Variation of static torque coefficients at different rotor angles at $\operatorname{Re}=1.98 \times 10^{5}$ for energy harvester with D2525-7525 cross sectional profile shape.

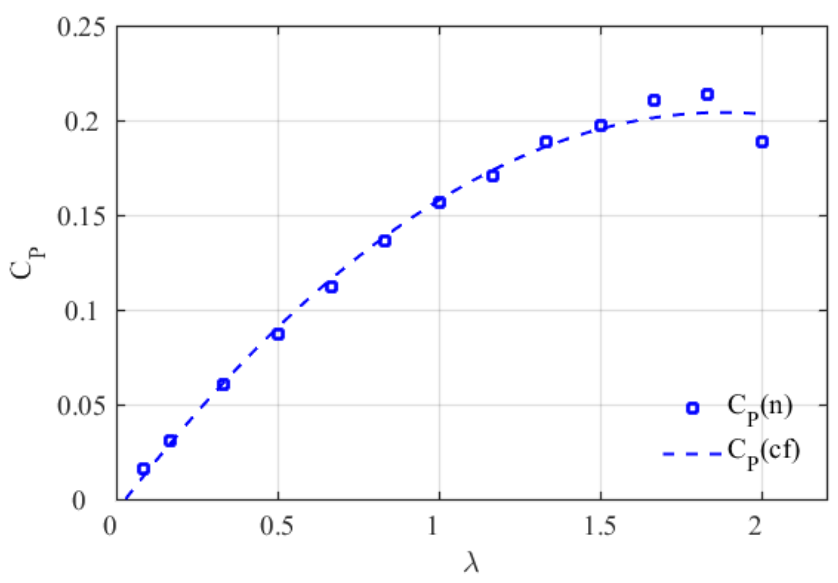

Fig.112 Performance characteristic curves for energy harvester with D2525-7525 cross sectional profile shape, at $R e=1.49 \times 10^{5}$.(c-f denotes curve fitting and $n$ denotes numerical results). 
The power coefficient for D2525-7525 model at different tip speed ratios can be found in Fig.112. Although this model has higher maximum power coefficient of 0.213 , which is higher than that of harvester $\mathrm{J}$, it requires high tip speed ratio $(\lambda=1.833)$ for this to happen. Due to the difficulties to achieve high tip speed ratio, D2525-7525 model is not expected to harness energy as efficiently as harvester $\mathbf{J}$ in practice.

For D2525-7525 model, it is expected to have similar self-starting capability as harvester J. However, its energy conversion efficiency is expected to be lower than that of harvester $\mathbf{J}$.

\subsubsection{Concluding remarks}

Energy harvester models with different cross-sectional blade profiles shapes are numerically studied, including evaluation on both their static and dynamic performances. The performances of these energy harvesters are compared against harvester $\mathrm{J}$ (S5050), and the comparison results are summarized in Table 12. It can be seen altering blade cross-section blade shape can possibly improve energy harvester's static or dynamic performance, or both. Some energy harvester models have potential to harness energy at higher energy conversion efficiency, but only at much higher tip speed ratios. Amongst the present energy harvester models studied, the one with S5025 blade shape shows both improved static and dynamic performances, at practically attainable tip speed ratio. 
Table 12 Summary of numerically studied static and dynamic performances of energy harvesters with different blade cross-sectional profile shapes, as compared with harvester $\mathbf{J}$.

\begin{tabular}{|c|c|c|}
\hline $\begin{array}{c}\text { Harvester } \\
\text { model }\end{array}$ & Static performance & Dynamic performance \\
\hline $\mathrm{S} 2550$ & - & + \\
\hline S7550 & \# & - \\
\hline S5025 & + & + \\
\hline $\mathrm{S} 2525$ & - & $+^{*}$ \\
\hline S7525 & - & - \\
\hline D2550-7550 & - & + \\
\hline D2525-7525 & \# & - \\
\hline Remarks & $\begin{array}{r}\text { '+' denotes bette } \\
\text { '\#' denotes no cleat } \\
\text { '+ }+{ }^{*} \text {, denotes better perfo }\end{array}$ & $\begin{array}{l}\text { lance than Model } \mathrm{J} \text {. } \\
\text { t can be made without } \\
\text { tests. } \\
\text { ly at much higher tip speed }\end{array}$ \\
\hline
\end{tabular}




\subsection{Comparison with other air-driven energy harvesters}

\subsubsection{Comparison with miniature horizontal axis wind turbine}

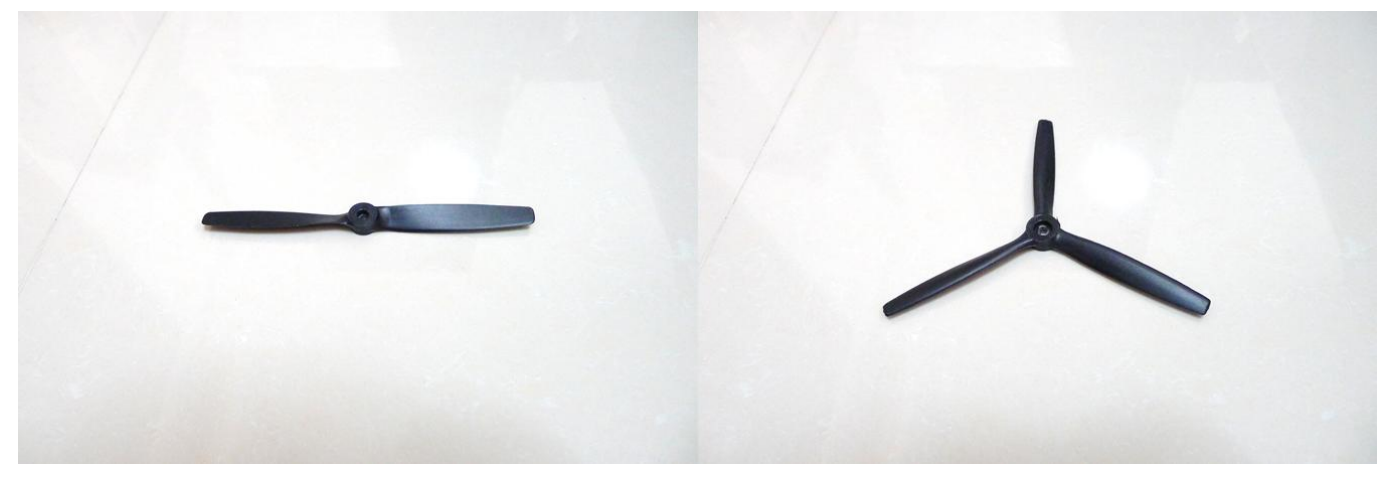

Fig.113 Two-blade and three-blade horizontal axis wind energy harvesters, both with diameter of 7 inch.

Further experiments are conducted to compare the performances of the current design and the conventional horizontal axis wind harvester. The horizontal axis wind harvesters (HAWHs) have different 1) number of blades (two blades or three blades), and 2) diameter of swept area (diameter=7 inch, 10 inch or 13 inch).

Fig.113 shows 2 examples of these horizontal axis wind harvesters with a diameter of 7-inch.

The performance of these HAWHs are also evaluated in the wind tunnel in Aerodynamics Laboratory in Nanyang Technological University, as they are installed on the same step height as described in Sect.3.2.2. Sentry ST372 IR thermo- Anemometer is used to monitor air flow speed at the vicinity of energy harvester. Electrical power output is measured and recorded by using Tektronix TDS1012B Two Channel Digital Storage Oscilloscope. 


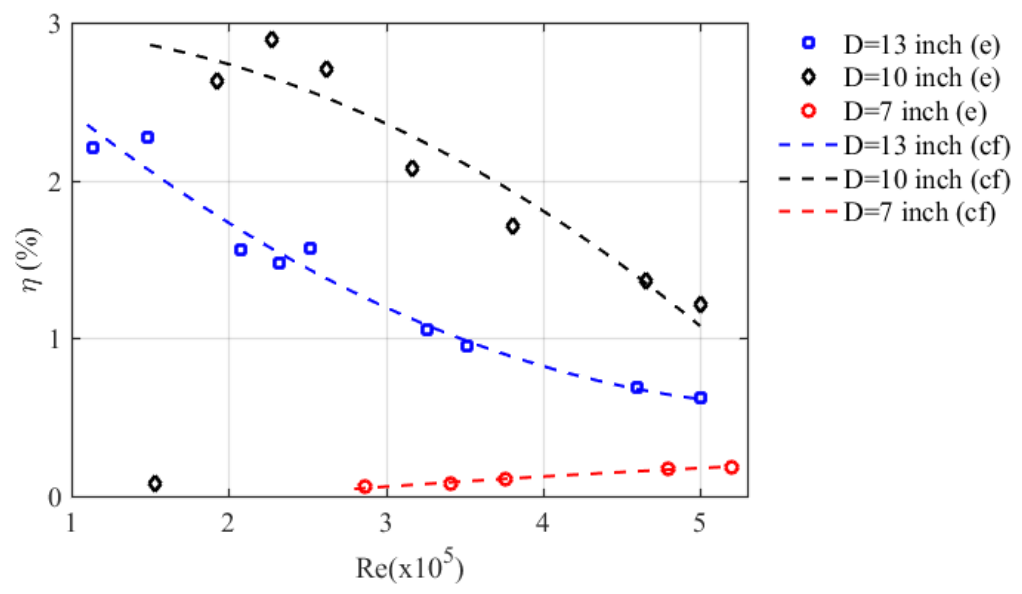

Fig.114 Overall energy conversion efficiencies varied with Reynolds number for 3blade HAWH with different swept area diameters. (c-f denotes curve fitting and e denotes experimental results).

Fig.114 shows the variation of overall energy conversion efficiencies with increasing Reynolds number for a 3-blade HAWH with different swept area diameters. The HAWH with a 10 -inch diameter is found to be involved with a higher overall energy conversion efficiency. Maximum overall energy conversion efficiency for the 3-blade HWAH is $\eta_{\max }=2.89 \%$ at $\mathrm{Re}=2.28 \times 10^{5}$. Furthermore, descending trends are observed for larger diameter HAWH (13 inch and 10 inch diameters), while an increasing trend is observed for the 7-inch HAWH.

The variation of the overall energy conversion efficiencies with Reynolds number for 2-blade HWAHs is shown in Figure115. It is found that the 10-inch HWAH is associated with higher overall energy conversion efficiency in comparison with other two different sizes of HWAHs. The maximum overall energy conversion efficiency for the 2-blade $\mathrm{HWAH}$ is $\eta_{\max }=3.21 \%$, at $\mathrm{Re}=1.93 \times 10^{5}$. It is much lower than the experimental maximum overall energy conversion efficiency 
of designed harvester $\mathbf{J}\left(\eta_{\max }=6.59 \%\right)$. Same descending trends are observed for 13-inch and 10-inch-diameter energy harvesters, and ascending trends is found for 7-inch energy harvester.

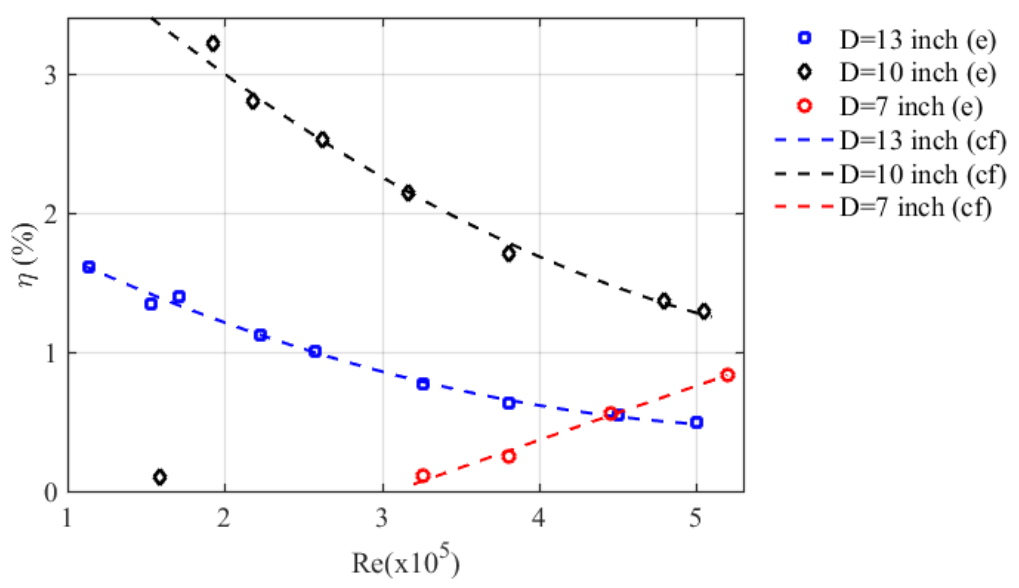

Figure115 Overall energy conversion efficiencies varied with Reynolds number for 3-blade HAWH with different swept area diameters. (c-f denotes curve fitting and e denotes experimental results).

\subsubsection{Comparison with other air-driven harvesters reported in literature}

The optimum design, energy harvester $\mathrm{J}$, has been experimentally tested and generates a total of electrical power approximately $0.305 \mathrm{~W}$ at Reynolds number around $5.10 \times 10^{5}$. The corresponding power density is approximately $30.51 \mathrm{~W} / \mathrm{m}^{2}$.The power density of the designed energy harvester is to be compared with other small-scale energy harvesters found in literature, as shown in Fig.116. 


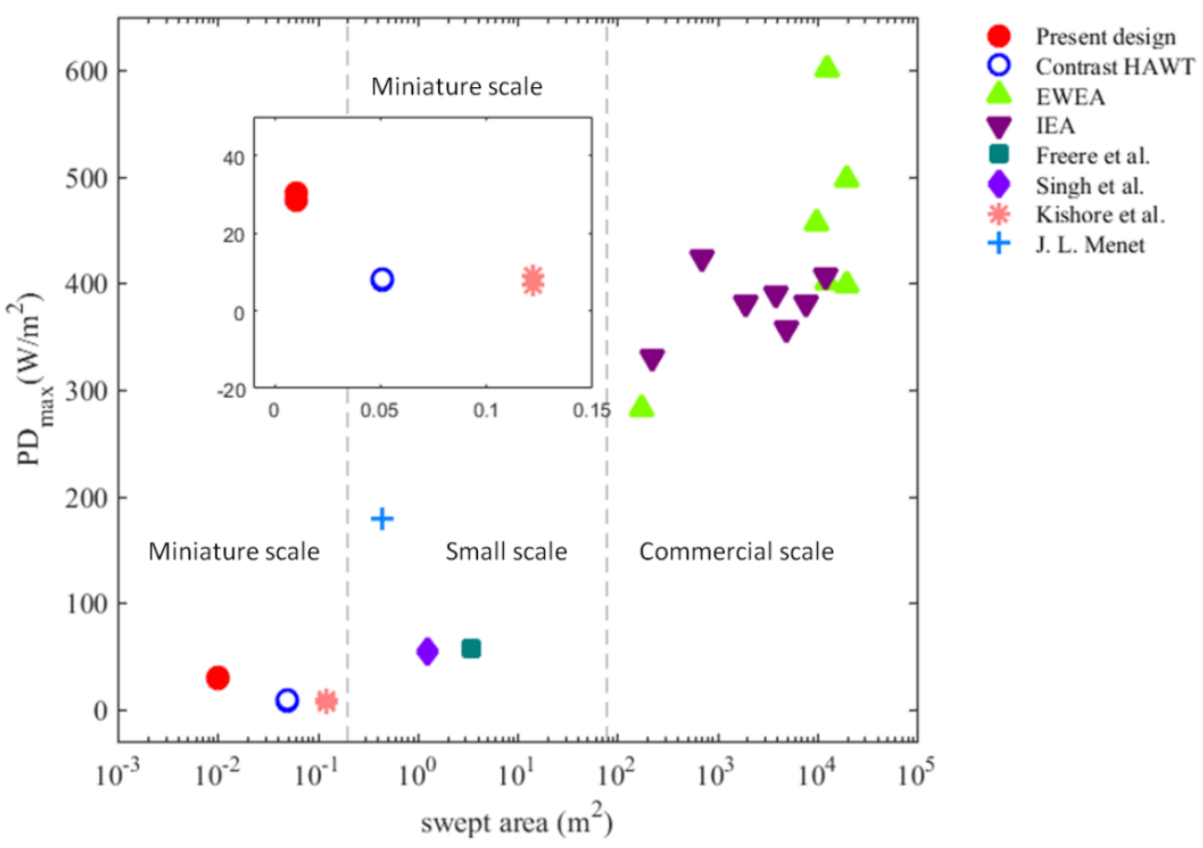

Fig.116 Comparison of maximum power density of other reported different scales energy harvesters. EWEA [91]. IEA [92]. Freere et al. [93]. Singh et al. [94]. Kishore et al. [95]. J. L. Menet. [96].

As the swept area is increased, the power density is increased with it. The commercial energy harvesters in large scale are more economical as they are taking advantage of large swept area. The power density of present designs is greater than the conventional vertical axis Savonius wind harvesters at micro scale. 


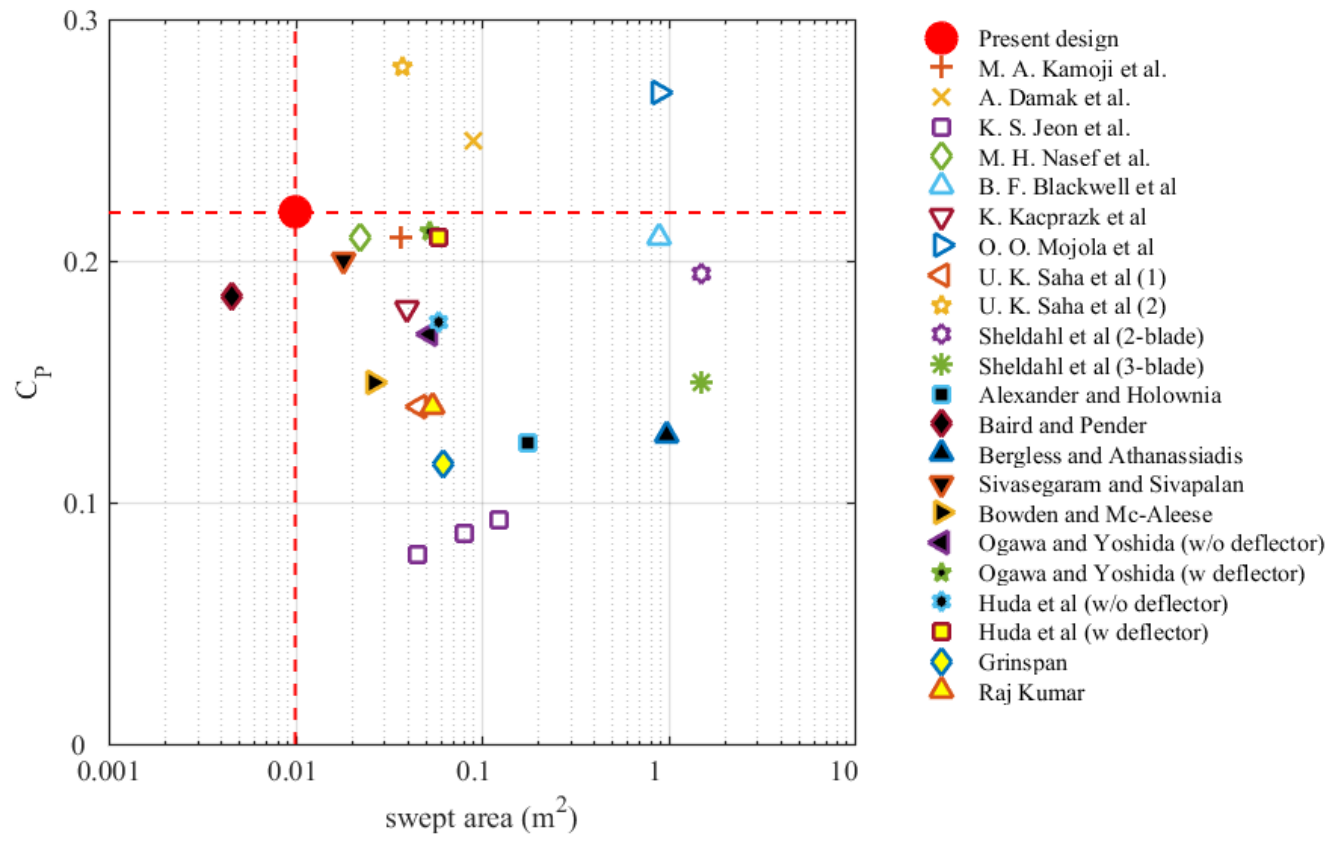

Fig.117Comparison of power coefficients of other reported energy harvesters at micro scale. Kamoji et al. [35]. Damak et al. [41]. Jeon et al. [42]. Nasef et al. [80]. Blackwell et al. [97]. Kacprazk et al. [98]. Mojola et al. [27] Saha et al (1) [33]. Saha et al (2) [34]. Sheldahl et al. (2-blade and 3-blade) [29]. Alexander and Holownia [30]. Baird and Pender [99]. Bergeless and Athanassiadis [100]. Sivasegaram and Sivapalan [101]. Bowden and Mc-Aleese [102]. Ogawa and Yoshida (without and with defector) [103]. Huda et al. (without and with defector) [104]. Grinspan [105] and Raj Kumar [106]

Power coefficient is a more commonly practiced way of discussing energy harvester efficiencies in literature. As an alternative way, the designed energy harvester is compared with other micro scale models in terms of power coefficient, as shown in Fig.117. It can be seen that many of the reported energy harvesters in literature works are larger in size, as compared to the present energy harvester. Most of the energy harvesters possess smaller or similar power coefficients. The value of power coefficient quoted for the present energy harvester model corresponds to the tip speed ratio that had been experimentally attained in practice for harvester $\mathrm{J}$, which is $C_{p}=0.22$. For those exhibited higher power coefficient, 
they have significantly larger size as compared to the present energy harvester. To the best knowledge of the author, very few studies are performed and reported on such small-size energy harvester, and the reported one are associated with power density lower than $10 \mathrm{~W} / \mathrm{m}^{2}$. Therefore, the present Savonius-like harvester with a maximum power density $30.51 \mathrm{~W} / \mathrm{m}^{2}$ and a maximum energy conversion efficiency of $6.59 \%$, is a simple and energy-efficient miniature harvester to harness mechanical energy from air flow. 


\section{Chapter 5. Conclusions and future works}

\subsection{Conclusions}

The present work considers the design, fabrication and evaluation of miniature wind energy harvesters, which have great potential to be applied in HVAC systems to harness and utilize 'man-made wind'. The motivation is an increasing concern for fossil fuel depletion and environmental sustainability. The focus of current energy research is to explore renewable and sustainable sources. Among these 'green and renewable energy' sources, wind power has great potential to be harnessed. Commercial onshore and offshore wind farms have been well built and operated. However, to the best knowledge of the author, systematic design and studies on miniature (swept area $<1 \mathrm{~m}^{2}$ ) wind energy harvesters have not been extensively conducted. For this, both experimental and numerical investigations are performed. 3D printing technology is exploited in this work as a fast manufacturing approach for prototype design of miniature energy harvesters driven by air flow. There are 10 energy harvesters designed and fabricated, which are labeled from harvester $\mathrm{A}$ to $\mathrm{J}$.

To determine the optimum design corresponding to maximum energy conversion efficiency, these harvesters are used to conduct parametric measurements to study the effects of 1) the number $\mathrm{N}$ of blades of each harvester, 2) the geometric size (characterized by SR) of each harvester, 3) energy harvester's aspect ratio (AR), 4) type of central part, 5) end plates and 6) air-driven energy harvester orientation. To 
simulate the experiments, 3D numerical simulations are conducted by solving Navier-stokes equations. In addition, the static and dynamic performances of these miniature harvesters are evaluated and compared. The series of static performance study reveals that:

- Modifying the harvester's length, diameter, number of blades and installation orientation results in little improvement on its static performance.

- Increasing Reynolds number does not lead to significant enhancement on static performance.

- Creation of additional air pathways such as removing solid central shaft or end plates improves the harvester's static performance by reducing undesirable high pressure at convex side of blades.

- The static performance of the harvester is determined by the pressure distribution pattern, which is jointly determined by the harvester geometric structure and its relative position with oncoming air flow (rotor angle). The pressure distribution pattern reveals that undesirable negative static torque coefficient is resulted from the high-pressure region at convex side of blade, while the desirable positive static torque coefficient value is mostly contributed by the high-pressure region at concave side.

- For a low cut-in Reynolds number to be achieved in practice, positive static torque coefficients at all rotor angles is plausible.

The systematic dynamic performance study reveals that: 
- Concentrating pressure at concave side of the blade is critical to achieve better dynamic performance, and an air pathway that releases the high pressure at concave side of blade should be avoided.

- Different from conventional Savonius-type wind turbines, the designed harvester is orientation-sensitive. Wider space between the blade and the large harvester diameter is necessary for 'anti-clockwise' to be utilized for higher energy conversion efficiency. Installation location of the harvester needs to be optimized, considering the combined effects of different design parameters and harvester orientation. The optimized location and orientation can lead to further improvement of the energy conversion efficiency of the harvester.

- For a harvester to be applied practically, its instantaneous dynamic torque coefficient is desired to be positive at all rotor angles.

The parametric measurements and simulations show that the optimum design of the energy harvester corresponding to maximum experimental energy conversion efficiency is the one with 3-blades,an aspect ratio of 1 , solid central shaft and end plates. The maximum energy conversion efficiency is approximately $6.59 \%$. Further study illustrates that 'clockwise' and 'anti-clockwise' orientated harvesters can produce different electrical power outputs due to interaction with step height platform. Therefore, a case study on energy harvester orientation is conducted. It is found that: 
- Changing the rotating orientation does not significantly affect the static performance of the energy harvesters.

- In order to better utilize 'anti-clockwise' orientation, the harvester diameter should be large enough to capture upward air flow. The blade number should not be large, as air flows may be hindered. However, fewer blades will enable pressure to be less effectively concentrated at the concave side of blade.

- Numerically predicted dynamic torque coefficients can be used to determine the preferred orientation. Harvester design with higher maximum dynamic torque coefficient and less negative minimum dynamic torque coefficient will be able to achieve higher energy conversion efficiency.

- The installation location needs to be optimized to achieve larger energy conversion efficiency. Further study is needed, and this is proposed in the future work.

The preliminary and orientation case studies of the miniature energy harvesters are concerned with blades in the shape of simple semi-cylindrical one. To gain insight on the blade shape effect, 3D numerical simulations are performed, by altering blade shape but keeping the basic geometric structure from the optimum design previously obtained $(\mathrm{N}=3, \mathrm{SR}=1, \mathrm{AR}=1$, with solid central shaft and end plates, 'anti-clockwise' orientation). The numerical study shows some possible improved designs of blade shape, but some of them with improved energy conversion efficiency can only be achieved at higher tip speed ratios. Among the blade shapes included in the present study, the energy harvester with S5025 shape (the highest 
point of blade is located at the middle point of the chord length, but closer to the chord) improves maximum static torque coefficient by $16 \%$, and maximum power coefficient is improved from 0.193 to 0.294 , as compared to Model J. Hence, both static and dynamic performance of designed harvester can be further improved.ss

involves with improved static performance and higher energy conversion efficiency.

In summary, the present study identifies the optimum miniature energy harvester design. It is the three-blade harvester with solid central shaft, end plates and aspect ratio of one installed in 'anti-clockwise' orientation. The maximum overall energy conversion efficiency is approximately $\eta=6.59 \%$. Numerical study results reveal that the miniature harvester's static and dynamic performance could be further improved, if the highest point of blade shape is brought closer to the blade chord.

\subsection{Future works}

In order to gain insights on the performance of these energy harvesters and to further improve their performance, further research is needed. These are listed below:

1) The installation location of the energy harvester needs to be optimized. A few parameters can be considered for determining the installation orientation of the energy harvester: a) the height of the step height platform,

b) the distance of energy harvester to the step height platform front edge 
and c) the distance that the energy harvester is lifted above the top surface of step height platform. The main objective is to place the energy harvester at the location associated with larger air flow rate.

2) More complicated energy harvester designs can be explored. Some possible improvements on the energy harvester's design are: a) twisted energy harvester blade, b) arranging a number of energy harvesters into an array, and c) adding auxiliary parts so that air flow is better directed and focused to the harvesters.

3) $3 \mathrm{D}$ printing is shown to be effective in producing $\mathrm{CAD}$ design of miniature energy harvesters driven by air flow in a relatively short period with acceptable resolution. However, 3D printing techniques are associated with a few challenges. One of the challenges is that there are limited choices of printing materials. And this limits the prototype properties such as weight and mechanical strength. Meanwhile, the surface of the present 3D printed energy harvesters is not smooth enough, which may increase the flow friction/drag and decrease the energy harvesting/conversion capacity. Thus further surface treatment techniques may be needed.

4) As the optimal energy harvester geometric structure design is determined, the harvester material choice and relevant manufacturing process should be optimized.

5) Field testing, cost, and lifecycle analysis should be conducted. 


\section{References}

[1] World Energy Outlook 2015. International Energy Agency (IEA); 2015.

[2] Abbott D. Keeping the energy debate clean: how do we supply the world's energy needs? Proceedings of the IEEE. 2010;98(1):42-66.

[3] Ren P. Renewables 2015 global status report. REN21 Secretariat: Paris, France. 2015.

[4] Medium-Term Renewable Energy Market Report 2013: Market Analysis and Forecasts to 2018.: International Energy Agency (IEA); 2013.

[5] Medium-Term Renewable Energy Market Report 2015: Market Analysis and Forecasts to 2020.: International Energy Agency (IEA); 2015.

[6] Global Wind Report 2015 -- Annual market update.: Global Wind Energy Council; 2015.

[7] Global Wind Energy Outlook 2014.: Global Wind Energy Council.; 2014.

[8] Tong W. Wind power generation and wind turbine design: Wit Press, 2010.

[9] Hemami A. Wind turbine technology: Cengage Learning, 2012.

[10] Mathew S. Wind energy: fundamentals, resource analysis and economics: Springer, 2006. 
[11] Manwell JF, McGowan JG, Rogers AL. Wind energy explained: theory, design and application: John Wiley \& Sons, 2010.

[12] Eldridge FR. Wind machines: Van Nostrand Reinhold Co., 1980.

[13] Ragheb M, Ragheb AM. Wind turbines theory-the betz equation and optimal rotor tip speed ratio: INTECH Open Access Publisher, 2011.

[14] Roy S, Saha UK. Review on the numerical investigations into the design and development of Savonius wind rotors. Renewable and Sustainable Energy Reviews. 2013;24:73-83.

[15] Kumbernuss J, Jian C, Wang J, Yang H, Fu W. A novel magnetic levitated bearing system for Vertical Axis Wind Turbines (VAWT). Applied energy. 2012;90(1):148-53.

[16] Stankovic S, Campbell N, Harries A. Urban wind energy: Earthscan, 2009.

[17] "Annual Climatological Report-2014". Centre for Climate Research Singapore. Available at $\quad$ http://www.weather.gov.sg/wpcontent/uploads/2015/05/Annual_Climatological_Report-2014.pdf.

[18] Akwa JV, Vielmo HA, Petry AP. A review on the performance of Savonius wind turbines. Renewable and Sustainable Energy Reviews. 2012;16(5):3054-64.

[19] Modi V, Fernando M. On the performance of the Savonius wind turbine. Journal of solar energy engineering. 1989;111(1):71-81. 
[20] Vedavarz, Ali Kumar, Sunil Hussain, Muhammed Iqbal. (2007). HVAC - The Handbook of Heating, Ventilation and Air Conditioning for Design and Implementation. Chapter 8, Duct Design. Industrial Press. Online version available at: $\quad$ http://app.knovel.com/hotlink/toc/id:kpHVACTHH2/hvac-handbookheating/hvac-handbook-heating.

[21] Savonius SJ. The S-rotor and its applications. Mech Eng. 1931;53:333-8.

[22] Fernando M, Modi V. A numerical analysis of the unsteady flow past a Savonius wind turbine. Journal of Wind Engineering and Industrial Aerodynamics. 1989;32(3):303-27.

[23] Fujisawa N. On the torque mechanism of Savonius rotors. Journal of Wind Engineering and Industrial Aerodynamics. 1992;40(3):277-92.

[24] Nakajima M, Iio S, Ikeda T. Performance of Savonius rotor for environmentally friendly hydraulic turbine. Journal of Fluid Science and Technology. 2008;3(3):420-9.

[25] Nakajima M, Iio S, Ikeda T. Performance of Double-step Savonius Rotor for Environmentally Friendly Hydraulic Turbine. Journal of Fluid Science and Technology. 2008;3:410-9.

[26] Simonds M, Bodek A. Performance test of a Savonius rotor: Brace Research Institute, Macdonald College of McGill University, 1964. 
[27] Mojola O. On the aerodynamic design of the Savonius windmill rotor. Journal of Wind Engineering and Industrial Aerodynamics. 1985;21(2):223-31.

[28] Rabah K, Osawa B. Design and field testing Savonius wind pump in East Africa. International journal of ambient energy. 1996;17(2):89-94.

[29] Sheldahl RE, Feltz L, Blackwell BF. Wind tunnel performance data for twoand three-bucket Savonius rotors. Journal of Energy. 1978;2(3):160-4.

[30] Alexander A, Holownia B. Wind tunnel tests on a Savonius rotor. Journal of Wind Engineering and Industrial Aerodynamics. 1978;3(4):343-51.

[31] Shankar P. Development of vertical axis wind turbines. Proceedings of the Indian Academy of Sciences Section C: Engineering Sciences. 1979;2(1):49-66.

[32] Hayashi T, Li Y, Hara Y. Wind tunnel tests on a different phase three-stage Savonius rotor. JSME International Journal Series B Fluids and Thermal Engineering. 2005;48(1):9-16.

[33] Saha U, Rajkumar MJ. On the performance analysis of Savonius rotor with twisted blades. Renewable energy. 2006;31(11):1776-88.

[34] Saha U, Thotla S, Maity D. Optimum design configuration of Savonius rotor through wind tunnel experiments. Journal of Wind Engineering and Industrial Aerodynamics. 2008;96(8):1359-75. 
[35] Kamoji M, Kedare SB, Prabhu S. Experimental investigations on single stage modified Savonius rotor. Applied Energy. 2009;86(7):1064-73.

[36] Kamoji M, Kedare S, Prabhu S. Experimental investigations on the effect of overlap ratio and blade edge conditions on the performance of conventional Savonius rotor. Wind Engineering. 2008;32(2):163-78.

[37] Roy S, Saha UK. Wind tunnel experiments of a newly developed two-bladed Savonius-style wind turbine. Applied Energy. 2015;137:117-25.

[38] Driss Z, Mlayeh O, Driss S, Driss D, Maaloul M, Abid MS. Study of the bucket design effect on the turbulent flow around unconventional Savonius wind rotors. Energy. 2015;89:708-29.

[39] Driss Z, Mlayeh O, Driss S, Maaloul M, Abid MS. Study of the incidence angle effect on the aerodynamic structure characteristics of an incurved Savonius wind rotor placed in a wind tunnel. Energy. 2016;113:894-908.

[40] Kamoji M, Kedare S, Prabhu S. Performance tests on helical Savonius rotors. Renewable Energy. 2009;34(3):521-9.

[41] Damak A, Driss Z, Abid M. Experimental investigation of helical Savonius rotor with a twist of 180. Renewable Energy. 2013;52:136-42. 
[42] Jeon KS, Jeong JI, Pan J-K, Ryu K-W. Effects of end plates with various shapes and sizes on helical Savonius wind turbines. Renewable Energy. 2015;79:167-76.

[43] Golecha K, Eldho T, Prabhu S. Influence of the deflector plate on the performance of modified Savonius water turbine. Applied Energy. 2011;88(9):3207-17.

[44] Mohamed M, Janiga G, Pap E, Thévenin D. Optimal blade shape of a modified Savonius turbine using an obstacle shielding the returning blade. Energy Conversion and Management. 2011;52(1):236-42.

[45] Altan BD, Altan G, Kovan V. Investigation of 3D printed Savonius rotor performance. Renewable Energy. 2016;99:584-91.

[46] Roy S, Saha UK. An adapted blockage factor correlation approach in wind tunnel experiments of a Savonius-style wind turbine. Energy Conversion and Management. 2014;86:418-27.

[47] Bhuyan S, Biswas A. Investigations on self-starting and performance characteristics of simple $\mathrm{H}$ and hybrid $\mathrm{H}$-Savonius vertical axis wind rotors. Energy Conversion and Management. 2014;87:859-67.

[48] Gupta R, Biswas A, Sharma K. Comparative study of a three-bucket Savonius rotor with a combined three-bucket Savonius-three-bladed Darrieus rotor. Renewable Energy. 2008;33(9):1974-81. 
[49] Wilson R, Lissaman P, Walker S. Aerodynamic performance of wind turbines. NASA STI/Recon Technical Report N. 1976;77:18598.

[50] Van Dusen E, Kirchhoff R. A two dimensional vortex sheet model of a savonius rotor. Conference A two dimensional vortex sheet model of a savonius rotor, vol. 1. p. 15-31.

[51] Jaohindy P, McTavish S, Garde F, Bastide A. An analysis of the transient forces acting on Savonius rotors with different aspect ratios. Renewable energy. 2013;55:286-95.

[52] Zhou T, Rempfer D. Numerical study of detailed flow field and performance of Savonius wind turbines. Renewable energy. 2013;51:373-81.

[53] McTavish S, Feszty D, Sankar T. Steady and rotating computational fluid dynamics simulations of a novel vertical axis wind turbine for small-scale power generation. Renewable energy. 2012;41:171-9.

[54] Shigetomi A, Murai Y, Tasaka Y, Takeda Y. Interactive flow field around two Savonius turbines. Renewable energy. 2011;36(2):536-45.

[55] El-Baz A, Youssef K, Mohamed M. Innovative improvement of a drag wind turbine performance. Renewable Energy. 2016;86:89-98. 
[56] Tartuferi M, D'Alessandro V, Montelpare S, Ricci R. Enhancement of Savonius wind rotor aerodynamic performance: a computational study of new blade shapes and curtain systems. Energy. 2015;79:371-84.

[57] Altan BD, Atılgan M. An experimental and numerical study on the improvement of the performance of Savonius wind rotor. Energy Conversion and Management. 2008;49(12):3425-32.

[58] Kawamura T, Sato Y. Numerical simulation of the flow around across-flow wind turbine. Research Institute of Mathematical Science, Kyoto University, Japan. 2002;1288(5):44-51.

[59] Zullah MA, Prasad D, Ahmed MR, Lee Y-H. Performance analysis of a wave energy converter using numerical simulation technique. Science in China Series E: Technological Sciences. 2010;53(1):13-8.

[60] Abraham J, Mowry G, Plourde B, Sparrow E, Minkowycz W. Numerical simulation of fluid flow around a vertical-axis turbine. Journal of Renewable and Sustainable Energy. 2011;3(3):033109.

[61] Saha UK, Rajkumar MJ, Maity D. Simulation of flow around and behind a Savonius rotor. International Energy Journal. 2005;6(2):83-90.

[62] Yaakob OB, Tawi K, Sunanto DS. Computer simulation studies on the effect overlap ratio for savonius type vertical axis marine current turbine. Int J Eng Trans A Basics. 2010;23:79-88. 
[63] Ogawa T. Theoretical study on the flow about Savonius rotor. Journal of fluids engineering. 1984;106(1):85-91.

[64] Modi V, Fernando M. Unsteady aerodynamics and wake of the Savonius wind turbine: a numerical study. Journal of Wind Engineering and Industrial Aerodynamics. 1993;46:811-6.

[65] Kotb M, Aldoss T. Flowfield around a partially-blocked Savonius rotor. Applied Energy. 1991;38(2):117-32.

[66] Fujisawa N. Velocity measurements and numerical calculations of flow fields in and around Savonius rotors. Journal of wind engineering and industrial aerodynamics. 1996;59(1):39-50.

[67] Afungchui D, Kamoun B, Helali A, Djemaa AB. The unsteady pressure field and the aerodynamic performances of a Savonius rotor based on the discrete vortex method. Renewable Energy. 2010;35(1):307-13.

[68] D'Alessandro V, Montelpare S, Ricci R, Secchiaroli A. Unsteady Aerodynamics of a Savonius wind rotor: a new computational approach for the simulation of energy performance. Energy. 2010;35(8):3349-63.

[69] Launder BE, Spalding D. The numerical computation of turbulent flows. Computer methods in applied mechanics and engineering. 1974;3(2):269-89. 
[70] Pope K, Rodrigues V, Doyle R, Tsopelas A, Gravelsins R, Naterer G, et al. Effects of stator vanes on power coefficients of a zephyr vertical axis wind turbine. Renewable Energy. 2010;35(5):1043-51.

[71] Debnath BK, Biswas A, Gupta R. Computational fluid dynamics analysis of a combined three-bucket Savonius and three-bladed Darrieus rotor at various overlap conditions. Journal of Renewable and Sustainable energy. 2009;1(3):033110.

[72] Gupta R, Biswas A. CFD analysis of flow physics and aerodynamic performance of a combined three-bucket Savonius and three-bladed Darrieus turbine. International Journal of Green Energy. 2011;8(2):209-33.

[73] Debnath BK, Biswas A, Gupta R. CFD analysis of three-bucket Savonius rotor using Fluent package. Conference CFD analysis of three-bucket Savonius rotor using Fluent package., NIT Silchar, India.

[74] Debnath BK, Biswas A, Gupta R. CFD analysis of combined three-bucket Savonius and three-bladed Darries rotor using Fluent package. Conference CFD analysis of combined three-bucket Savonius and three-bladed Darries rotor using Fluent package., SVNIT, Surat, India.

[75] Roy S, Saha U. Comparative analysis of turbulence models for flow simulation around a vertical axis wind turbine. Conference Comparative analysis of turbulence models for flow simulation around a vertical axis wind turbine. p. 22-3. 
[76] Menter FR. Zonal two equation k-turbulence models for aerodynamic flows. AIAA paper. 1993;2906:1993.

[77] Menter FR. Two-equation eddy-viscosity turbulence models for engineering applications. AIAA journal. 1994;32(8):1598-605.

[78] Plourde B, Abraham J, Mowry G, Minkowycz W. Simulations of threedimensional vertical-axis turbines for communications applications. Wind Engineering. 2012;36(4):443-53.

[79] Dobrev I, Massouh F. CFD and PIV investigation of unsteady flow through Savonius wind turbine. Energy Procedia. 2011;6:711-20.

[80] Nasef M, El-Askary W, AbdEL-Hamid A, Gad H. Evaluation of Savonius rotor performance: Static and dynamic studies. Journal of Wind Engineering and Industrial Aerodynamics. 2013;123:1-11.

[81] Feynman RP, Leighton RB, Sands ML. The Feynman lectures on physics. 1963.

[82] SolidWorks I. Solidworks corporation. Concord, MA. 2002.

[83] Prestech Industrial Automation Pte Ltd. Available at: www.prestech.com.sg.

[84] Dimitrov D, Schreve K, De Beer N. Advances in three dimensional printingstate of the art and future perspectives. Rapid Prototyping Journal. 2006;12(3):13647. 
[85] Wohlers TT, Caffrey T. Wohlers Report 2015: 3D Printing and Additive Manufacturing State of the Industry Annual Worldwide Progress Report: Wohlers Associates, 2015.

[86] Karapatis N, Van Griethuysen J, Glardon R. Direct rapid tooling: a review of current research. Rapid Prototyping Journal. 1998;4(2):77-89.

[87] MakerBot. Available at: http://www.makerbot.com.

[88] MakerBot Replicator 2 Desktop 3D Printer User Manual. MakerBot.

[89] William H, Alan P. Low Speed Wind Tunnel Testing. Jonh Wiley \& Sons. $1984 ; 61$.

[90] Ross I, Altman A. Wind tunnel blockage corrections: Review and application to Savonius vertical-axis wind turbines. Journal of Wind Engineering and Industrial Aerodynamics. 2011;99(5):523-38.

[91] European Wind Energy Association. European statistics. Brussels; 2011.

[92] International Energy Agency. World energy outlook. Paris, France; 2013.

[93] Freere P, Sacher M, Derricott J, Hanson B. A low cost wind turbine and blade performance. Wind Engineering. 2010;34(3):289-302.

[94] Singh RK, Ahmed MR. Blade design and performance testing of a small wind turbine rotor for low wind speed applications. Renewable Energy. 2013;50:812-9. 
[95] Kishore RA, Coudron T, Priya S. Small-scale wind energy portable turbine (SWEPT). Journal of Wind Engineering and Industrial Aerodynamics. $2013 ; 116: 21-31$.

[96] Menet J-L. A double-step Savonius rotor for local production of electricity: a design study. Renewable energy. 2004;29(11):1843-62.

[97] Blackwell BF, Sheldahl RF, Feltz LV. Wind tunnel performance data for twoand three-bucket Savonius rotors: Sandia Laboratories, 1977.

[98] Kacprzak K, Liskiewicz G, Sobczak K. Numerical investigation of conventional and modified Savonius wind turbines. Renewable energy. $2013 ; 60: 578-85$.

[99] Baird J, Pender S. Optimization of a vertical axis wind turbine for small scale applications. Conference Optimization of a vertical axis wind turbine for small scale applications. Institution of Engineers, Australia, p. 431.

[100] Bergeles G, Athanassiadis N. On the flow field around a Savonius rotor. Wind Engineering. 1982;6:140-8.

[101] Sivasegaram S, Sivapalan S. Augmentation of power in slow-running vertical-axis wind rotors using multiple vanes. Wind Engineering. 1983;7:12-9.

[102] Bowden G, McAleese S. The properties of isolated and coupled Savonius rotors. Wind Engineering. 1984;8:271-88. 
[103] OGAWA T, YOSHIDA H. The effects of a deflecting plate and rotor end plates on performances of Savonius-type wind turbine. Bulletin of JSME. 1986;29(253):2115-21.

[104] Huda M, Selim M, Islam A, Islam M. The performance of an S-shaped Savonius rotor with a deflecting plate. RERIC International Energy Journal. 1992;14(1):25-32.

[105] Grinspan A. Development of a Low Speed Wind Tunnel and Testing of Savonius Wind Turbine Rotor with Twisted Blades. M Tech Thesis, Department of Mechanical Engineering, IIT Guwahati, India. 2002.

[106] Rajkumar M. Experimental investigation and flow simulation of Savonius rotor. M. Tech: Thesis. India: Department of Mechanical Engineering, IIT Guwahati, 2004.

[107] Chua CK, Leong KF. 3D printing and additive manufacturing: principles and applications: World Scientific, 2015. Fused deposition modeling, https://en.wikipedia.org/wiki/Fused_deposition_modeling. 


\section{Appendix A: Energy harvester model design}

\section{Harvester A}

Table 13 Design of harvester A

\begin{tabular}{ll}
\hline Design factor & Description \\
\hline Number of bladed $(N)$ & 3 \\
Geometric size $(S R)$ & 1 \\
Aspect ratio $(A R)$ & 1 \\
Type of central part & Solid \\
Presence of end plates & Yes \\
Orientation & Lower \\
\hline
\end{tabular}

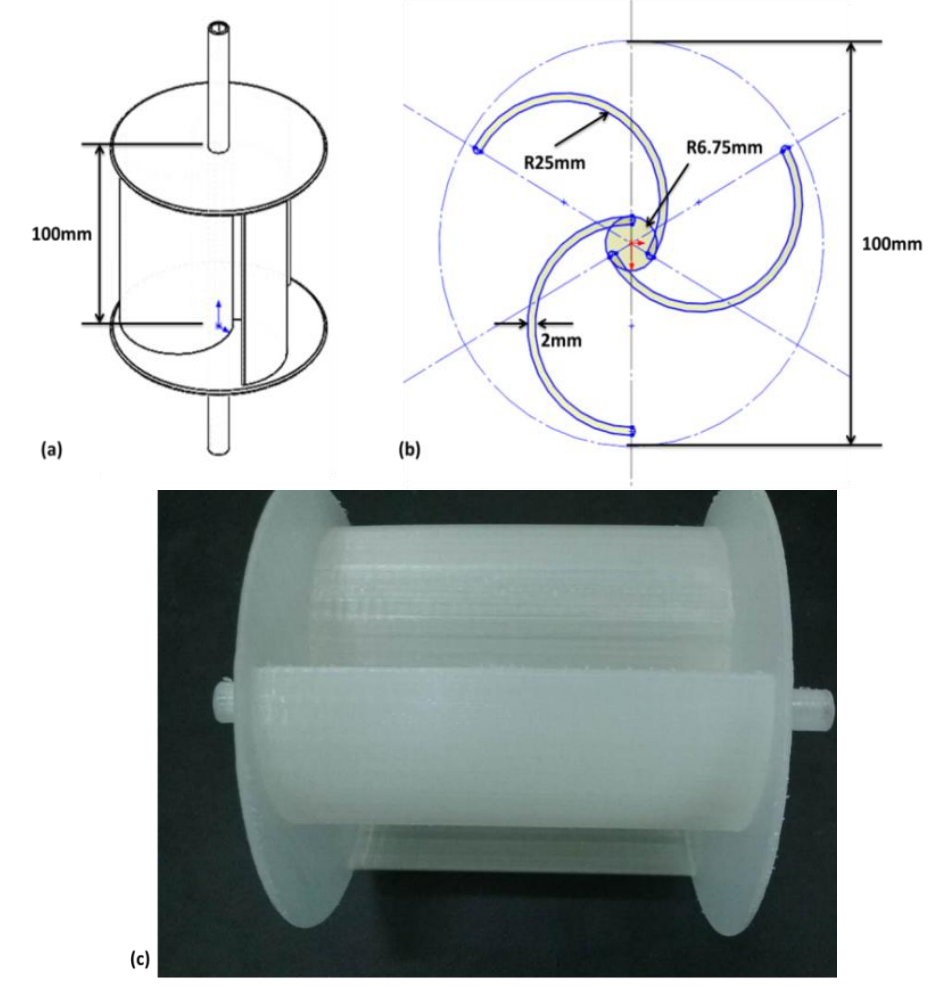

Fig.118 (a) 3D view, (b) Top view and (c) 3D printed model of harvester A 
Table 14 Characteristic parameters of harvester A

\begin{tabular}{ll}
\hline Parameter & Value \\
\hline Blade thickness & $2 \mathrm{~mm}$ \\
Blade overlap & $13.5 \mathrm{~mm}$ \\
Rotor diameter & $86.5 \mathrm{~mm}$ \\
End plate diameter & $100 \mathrm{~mm}$ \\
End plate thickness & $2 \mathrm{~mm}$ \\
Turbine length & $100 \mathrm{~mm}$ \\
\hline
\end{tabular}

\section{Harvester B}

Table 15 Design of harvester B

\begin{tabular}{ll}
\hline Design factor & Description \\
\hline Number of bladed $(N)$ & 2 \\
Geometric size $(S R)$ & 1 \\
Aspect ratio $(A R)$ & 1 \\
Type of central part & Solid \\
Presence of end plates & Yes \\
Orientation & Lower \\
\hline
\end{tabular}



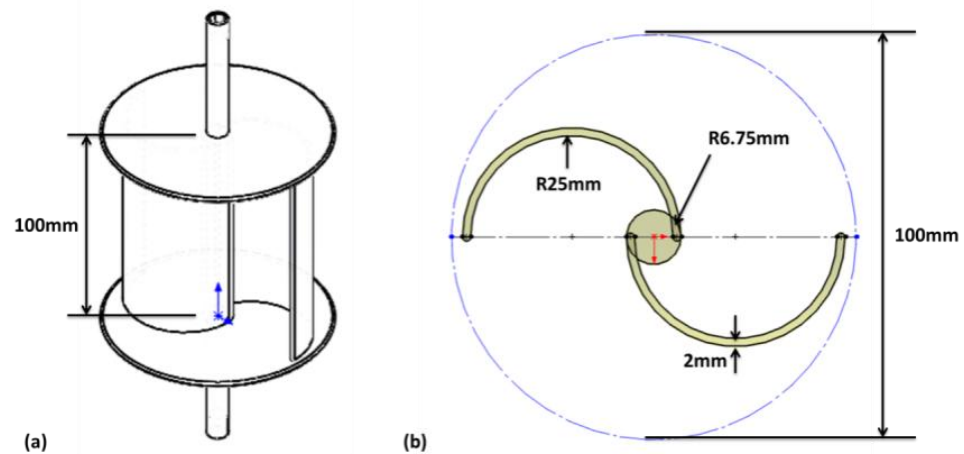

(b)

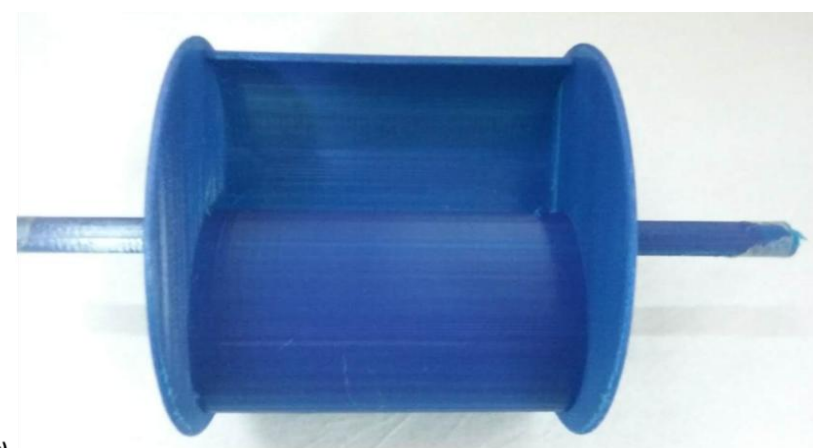

(c)

Fig.119 (a) 3D view, (b) Top view and (c) 3D printed model of harvester B

Table 16 Characteristic parameters of harvester B

\begin{tabular}{ll}
\hline Parameter & Value \\
\hline Blade thickness & $2 \mathrm{~mm}$ \\
Blade overlap & $13.5 \mathrm{~mm}$ \\
Rotor diameter & $86.5 \mathrm{~mm}$ \\
End plate diameter & $100 \mathrm{~mm}$ \\
End plate thickness & $2 \mathrm{~mm}$ \\
Turbine length & $100 \mathrm{~mm}$ \\
\hline
\end{tabular}




\section{Harvester C}

Table 17 Design of harvester C

\begin{tabular}{ll}
\hline Design factor & Description \\
\hline Number of bladed $(N)$ & 4 \\
Geometric size $(S R)$ & 1 \\
Aspect ratio $(A R)$ & 1 \\
Type of central part & Solid \\
Presence of end plates & Yes \\
Orientation & Lower
\end{tabular}

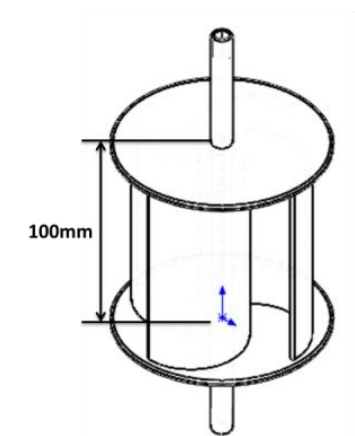

(a)

(b)

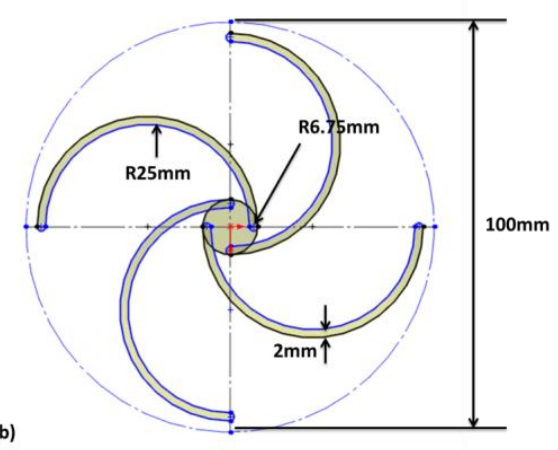

(c)

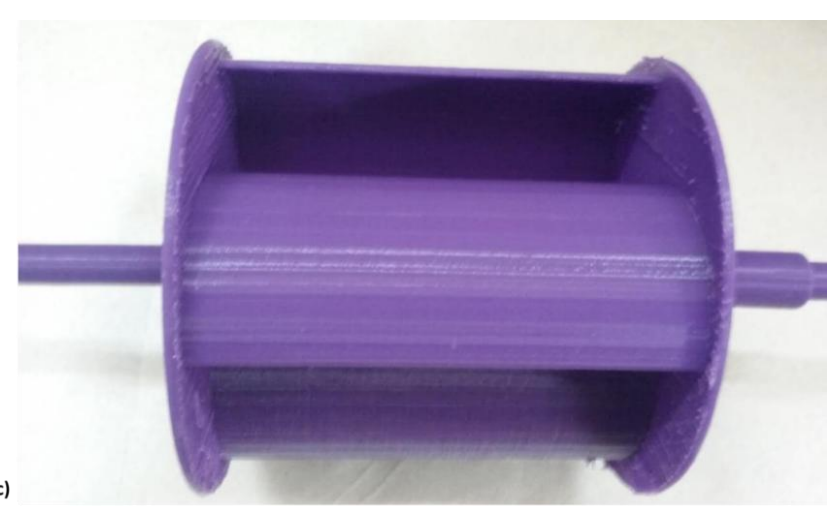

Fig.120 (a) 3D view, (b) Top view and (c) 3D printed model of harvester C 
Table 18 Characteristic Parameters of harvester C

\begin{tabular}{ll}
\hline Parameter & Value \\
\hline Blade thickness & $2 \mathrm{~mm}$ \\
Blade overlap & $13.5 \mathrm{~mm}$ \\
Rotor diameter & $86.5 \mathrm{~mm}$ \\
End plate diameter & $100 \mathrm{~mm}$ \\
End plate thickness & $2 \mathrm{~mm}$ \\
Turbine length & $100 \mathrm{~mm}$ \\
\hline
\end{tabular}

\section{Harvester D}

Table 19 Design of harvester D

\begin{tabular}{ll}
\hline Design factor & Description \\
\hline Number of bladed $(N)$ & 3 \\
Geometric size $(S R)$ & $2 / 3$ \\
Aspect ratio $(A R)$ & 1 \\
Type of central part & Solid \\
Presence of end plates & Yes \\
Orientation & Lower \\
\hline
\end{tabular}




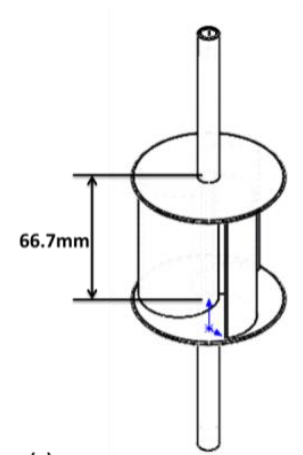

(a)
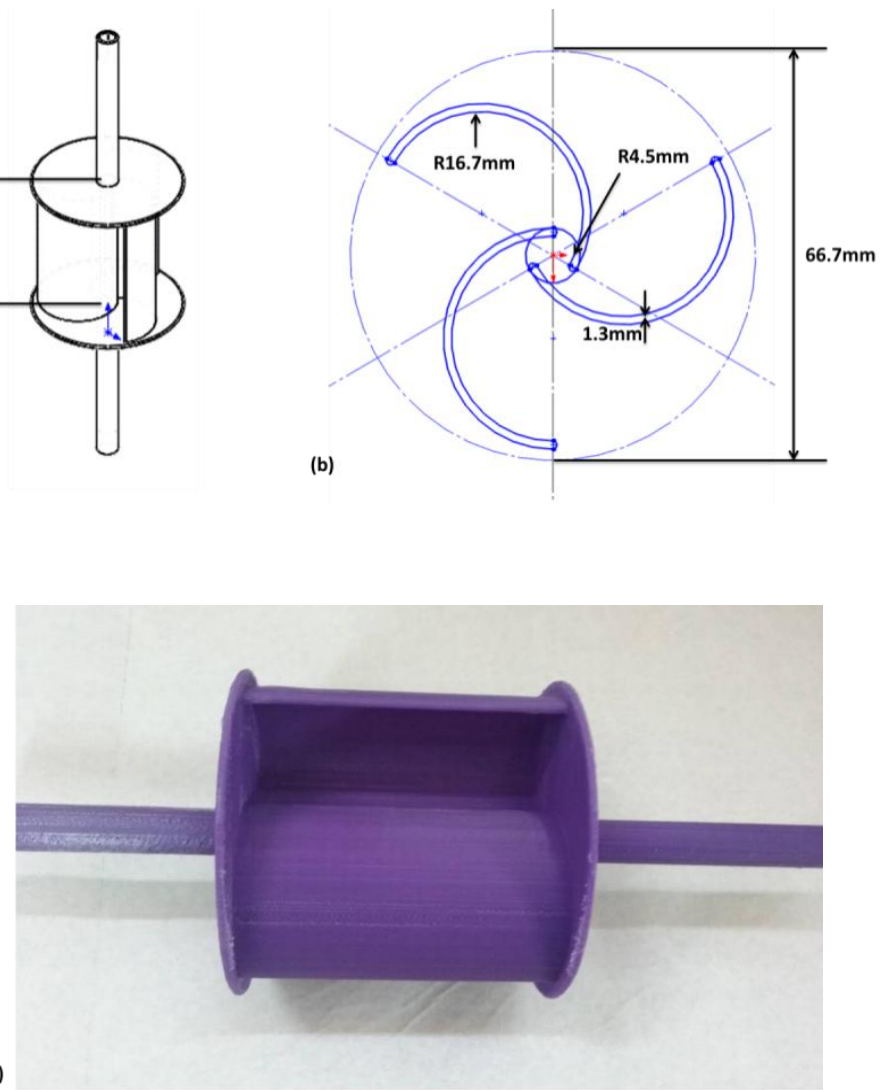

Fig.121 (a) 3D view, (b) Top view and (c) 3D printed model of harvester D

Table 20 Characteristic parameters of harvester D

\begin{tabular}{ll}
\hline Parameter & Value \\
\hline Blade thickness & $1.3 \mathrm{~mm}$ \\
Blade overlap & $9 \mathrm{~mm}$ \\
Rotor diameter & $57.7 \mathrm{~mm}$ \\
End plate diameter & $67.7 \mathrm{~mm}$ \\
End plate thickness & $1.3 \mathrm{~mm}$ \\
Turbine length & $67.7 \mathrm{~mm}$ \\
\hline
\end{tabular}




\section{Harvester E}

Table 21 Design of harvester $\mathbf{E}$

\begin{tabular}{ll}
\hline Design factor & Description \\
\hline Number of bladed $(N)$ & 3 \\
Geometric size $(S R)$ & $4 / 3$ \\
Aspect ratio $(A R)$ & 1 \\
Type of central part & Solid \\
Presence of end plates & Yes \\
Orientation & Lower
\end{tabular}
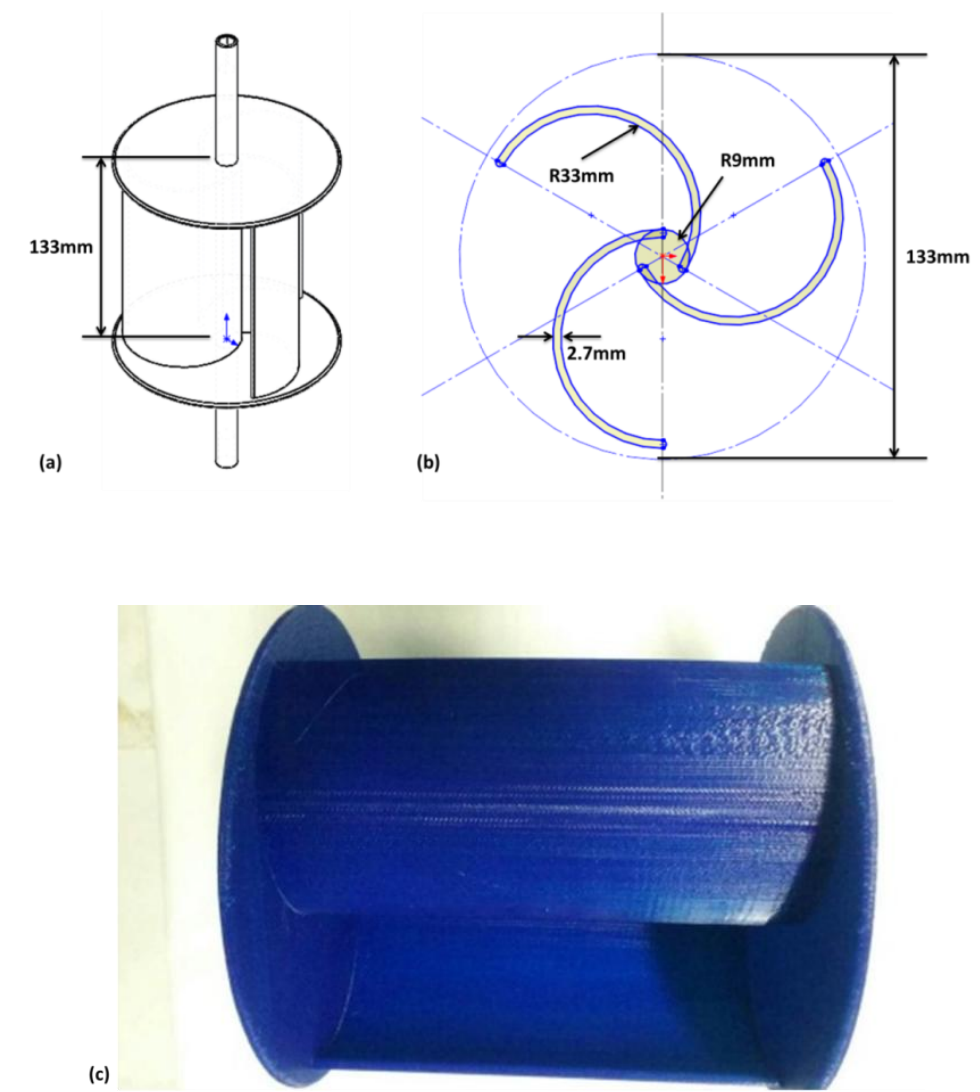

Fig.122 (a) 3D view, (b) Top view and (c) 3D printed model of harvester E 
Table 22 Characteristic parameters of harvester $\mathbf{E}$

\begin{tabular}{ll}
\hline Parameter & Value \\
\hline Blade thickness & $2.7 \mathrm{~mm}$ \\
Blade overlap & $9 \mathrm{~mm}$ \\
Rotor diameter & $115 \mathrm{~mm}$ \\
End plate diameter & $133 \mathrm{~mm}$ \\
End plate thickness & $2.7 \mathrm{~mm}$ \\
Turbine length & $133 \mathrm{~mm}$ \\
\hline
\end{tabular}

\section{Harvester F}

Table 23 Design of harvester $F$

\begin{tabular}{ll}
\hline Design factor & Description \\
\hline Number of bladed $(N)$ & 3 \\
Geometric size $(S R)$ & $1 / 2$ \\
Aspect ratio $(A R)$ & $1 / 2$ \\
Type of central part & Solid \\
Presence of end plates & Yes \\
Orientation & Lower \\
\hline
\end{tabular}




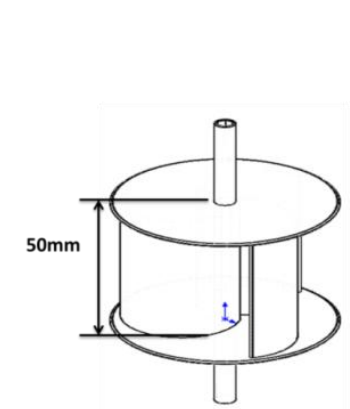

(a)

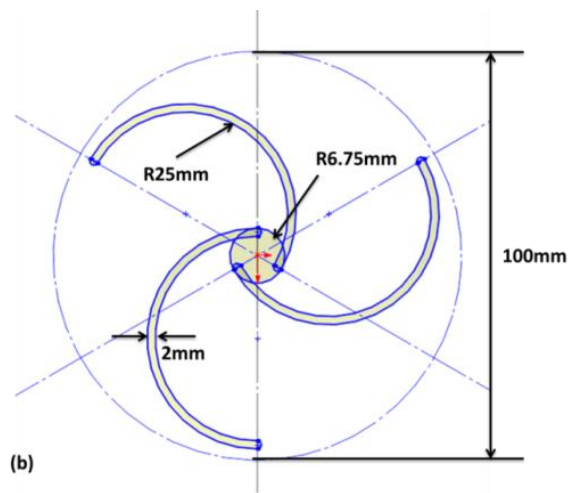

(c)

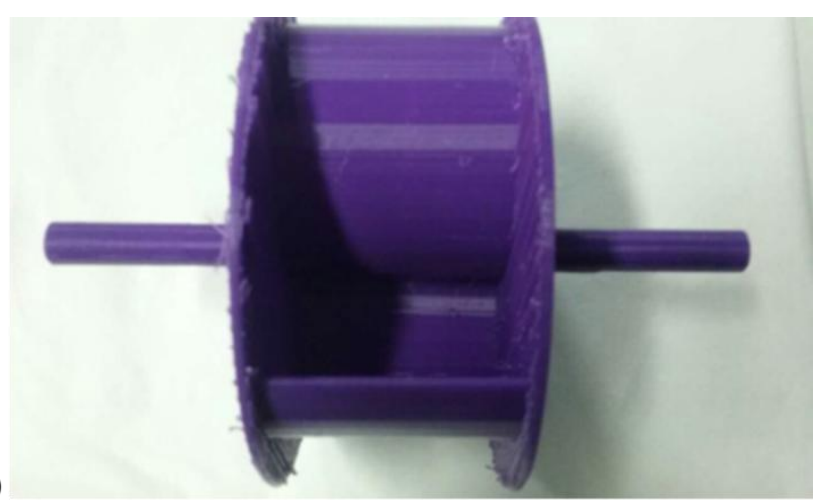

Fig.123 (a) 3D view, (b) Top view and (c) 3D printed model of harvester $F$

Table 24 Characteristic parameters of harvester $\mathbf{F}$

\begin{tabular}{ll}
\hline Parameter & Value \\
\hline Blade thickness & $2 \mathrm{~mm}$ \\
Blade overlap & $13.5 \mathrm{~mm}$ \\
Rotor diameter & $86.5 \mathrm{~mm}$ \\
End plate diameter & $100 \mathrm{~mm}$ \\
End plate thickness & $2 \mathrm{~mm}$ \\
Turbine length & $50 \mathrm{~mm}$ \\
\hline
\end{tabular}




\section{Harvester G}

Table 25 Design of harvester G

\begin{tabular}{ll}
\hline Design factor & Description \\
\hline Number of bladed $(N)$ & 3 \\
Geometric size $(S R)$ & $1 / 2$ \\
Aspect ratio $(A R)$ & 2 \\
Type of central part & Solid \\
Presence of end plates & Yes \\
Orientation & Lower
\end{tabular}

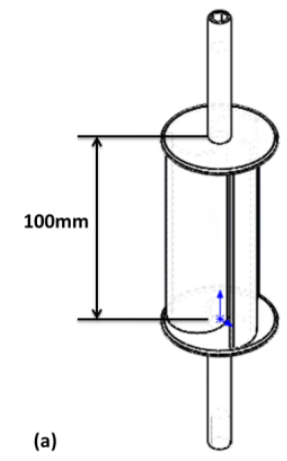

(b)
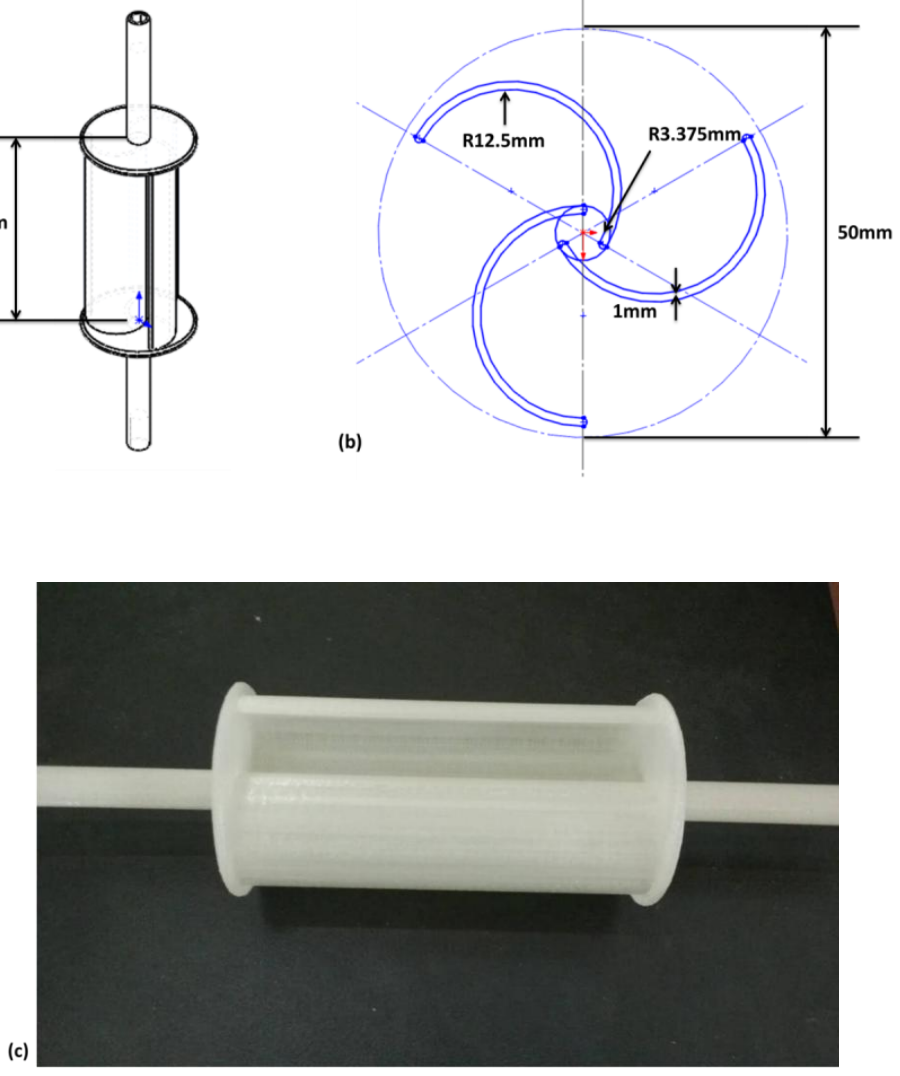

Fig.124 (a) 3D view, (b) Top view and (c) 3D printed model of harvester G 
Table 26 Characteristic parameters of harvester G

\begin{tabular}{ll}
\hline Parameter & Value \\
\hline Blade thickness & $1 \mathrm{~mm}$ \\
Blade overlap & $6.75 \mathrm{~mm}$ \\
Rotor diameter & $43.25 \mathrm{~mm}$ \\
End plate diameter & $50 \mathrm{~mm}$ \\
End plate thickness & $2 \mathrm{~mm}$ \\
Turbine length & $100 \mathrm{~mm}$ \\
\hline
\end{tabular}

\section{Harvester H}

Table 27 Design of harvester $\mathbf{H}$

\begin{tabular}{ll}
\hline Design factor & Description \\
\hline Number of bladed $(N)$ & 3 \\
Geometric size $(S R)$ & 1 \\
Aspect ratio $(A R)$ & 1 \\
Type of central part & Empty \\
Presence of end plates & Yes \\
Orientation & Lower \\
\hline
\end{tabular}



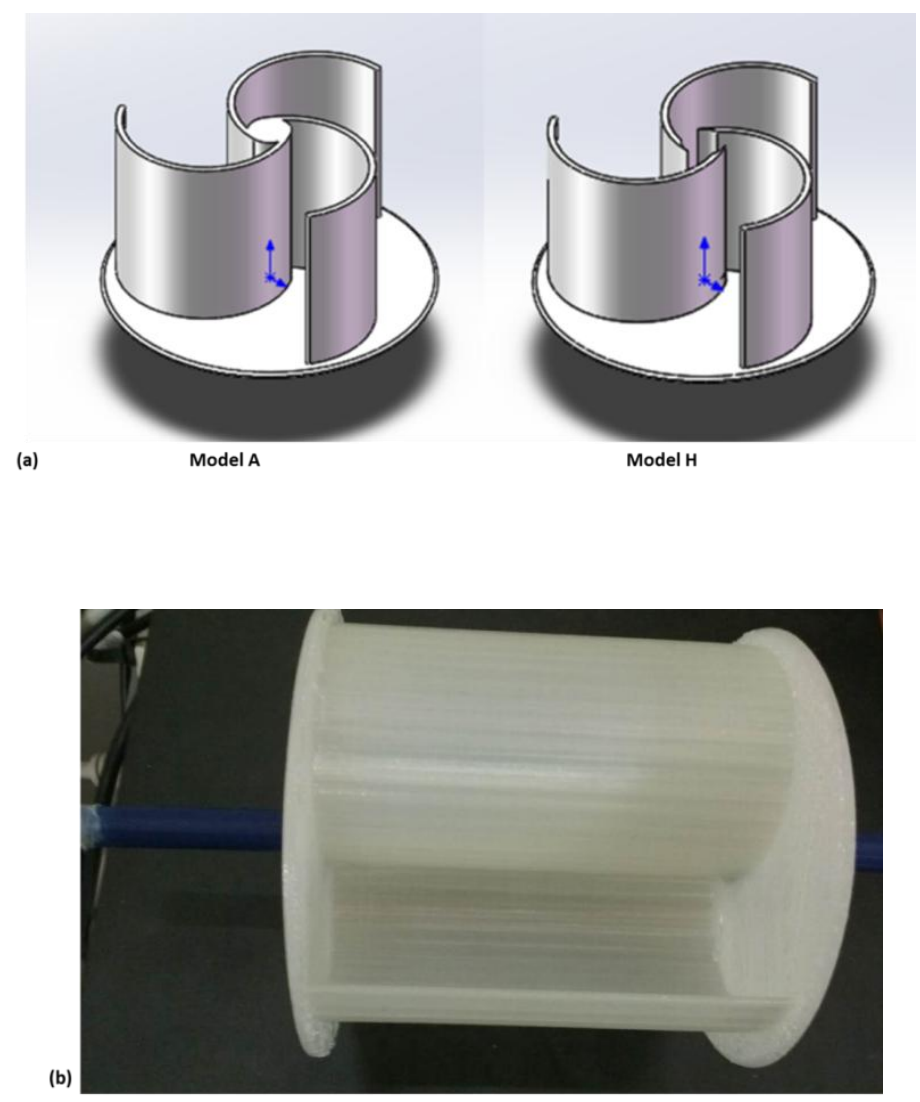

Fig.125 (a) Comparison between harvester $\mathrm{H}$ and A, and (b) 3D printed model of harvester $\mathbf{H}$

Table 28 Characteristic parameters of harvester $\mathbf{H}$

\begin{tabular}{ll}
\hline Parameter & Value \\
\hline Blade thickness & $2 \mathrm{~mm}$ \\
Blade overlap & $13.5 \mathrm{~mm}$ \\
Rotor diameter & $86.5 \mathrm{~mm}$ \\
End plate diameter & $100 \mathrm{~mm}$ \\
End plate thickness & $2 \mathrm{~mm}$ \\
Turbine length & $100 \mathrm{~mm}$ \\
\hline
\end{tabular}




\section{Harvester I}

Table 29 Design of harvester I

\begin{tabular}{ll}
\hline Design factor & Description \\
\hline Number of bladed $(N)$ & 3 \\
Geometric size $(S R)$ & 1 \\
Aspect ratio $(A R)$ & 1 \\
Type of central part & Solid \\
Presence of end plates & No \\
Orientation & Lower \\
\hline
\end{tabular}

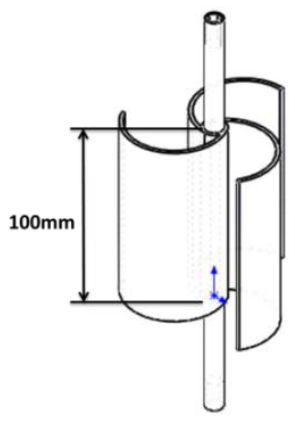

(a)

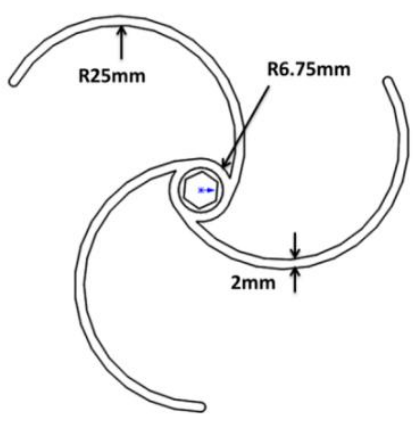

(b)

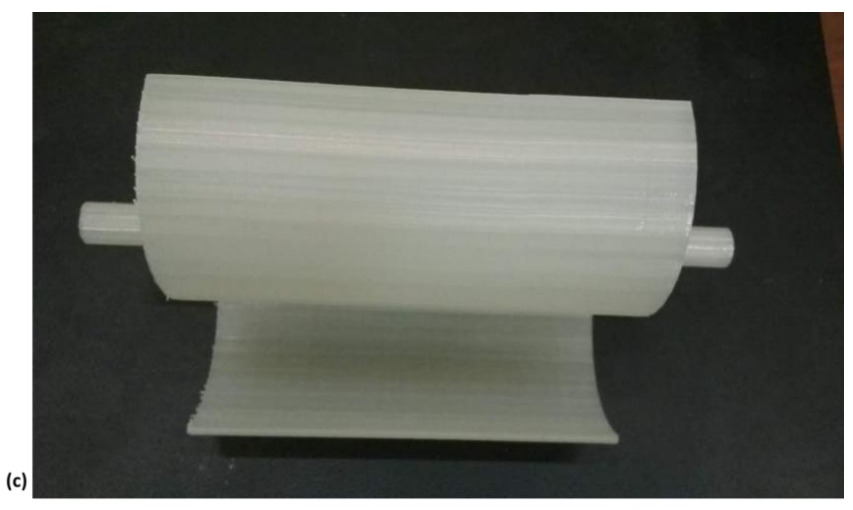

Fig.126 (a) 3D view, (b) Top view and (c) 3D printed model of harvester I 
Table 30 Characteristic parameters of harvester I

\begin{tabular}{ll}
\hline Parameter & Value \\
\hline Blade thickness & $2 \mathrm{~mm}$ \\
Blade overlap & $13.5 \mathrm{~mm}$ \\
Rotor diameter & $86.5 \mathrm{~mm}$ \\
Turbine length & $100 \mathrm{~mm}$ \\
\hline
\end{tabular}

\section{Harvester J}

Table 31 Design of harvester J

\begin{tabular}{ll}
\hline Design factor & Description \\
\hline Number of bladed $(N)$ & 3 \\
Geometric size $(S R)$ & 1 \\
Aspect ratio $(A R)$ & 1 \\
Type of central part & Solid \\
Presence of end plates & Yes \\
Orientation & Upper \\
\hline
\end{tabular}

Table 32 Characteristic parameters of harvester J

\begin{tabular}{ll}
\hline Parameter & Value \\
\hline Blade thickness & $2 \mathrm{~mm}$ \\
Blade overlap & $13.5 \mathrm{~mm}$ \\
Rotor diameter & $86.5 \mathrm{~mm}$
\end{tabular}


End plate diameter

End plate thickness

Turbine length
$100 \mathrm{~mm}$

$2 \mathrm{~mm}$

$100 \mathrm{~mm}$ 


\section{Appendix B: Wind tunnel results for preliminary parametric study}

\section{Harvester A}

Table 33 Experimental measurements results of energy harvester A

\begin{tabular}{ccc}
\hline Air flow speed $(\mathbf{m} / \mathbf{s})$ & RPM & Peak emf $(\mathbf{V})$ \\
\hline $\mathbf{4 . 2 3}$ & 115 & 0.357 \\
$\mathbf{4 . 6 7}$ & 178 & 0.636 \\
$\mathbf{5 . 0 6}$ & 219 & 0.824 \\
$\mathbf{5 . 1 2}$ & 236 & 0.884 \\
$\mathbf{5 . 7 7}$ & 303 & 1.175 \\
$\mathbf{6 . 5 8}$ & 643 & 1.841 \\
$\mathbf{7 . 4 4}$ & 877 & 3.451 \\
$\mathbf{8 . 7 8}$ & 1091 & 4.331 \\
$\mathbf{9 . 1 2}$ & 1174 & 4.767 \\
$\mathbf{9 . 7 6}$ & 1280 & 5.063 \\
$\mathbf{1 0 . 2 1}$ & 1284 & 5.176 \\
& & \\
\hline
\end{tabular}




\section{Harvester B}

Table 34 Experimental measurements results of energy harvester B

\begin{tabular}{ccc}
\hline Air flow speed $(\mathbf{m} / \mathbf{s})$ & RPM & Peak emf $(\mathbf{V})$ \\
\hline $\mathbf{5 . 6 5}$ & 229 & 0.97 \\
$\mathbf{5 . 8 5}$ & 323 & 1.166 \\
$\mathbf{6 . 9 8}$ & 589 & 2.251 \\
$\mathbf{7 . 6 3}$ & 659 & 2.319 \\
$\mathbf{8 . 3 4}$ & 728 & 2.553 \\
$\mathbf{8 . 3 7}$ & 755 & 2.635 \\
$\mathbf{8 . 9 8}$ & 753 & 2.645 \\
$\mathbf{9 . 7 3}$ & 756 & 2.672 \\
& & \\
\hline $\mathbf{1 0 . 1 3}$ & 758 & 2.671 \\
\hline & & \\
\hline
\end{tabular}



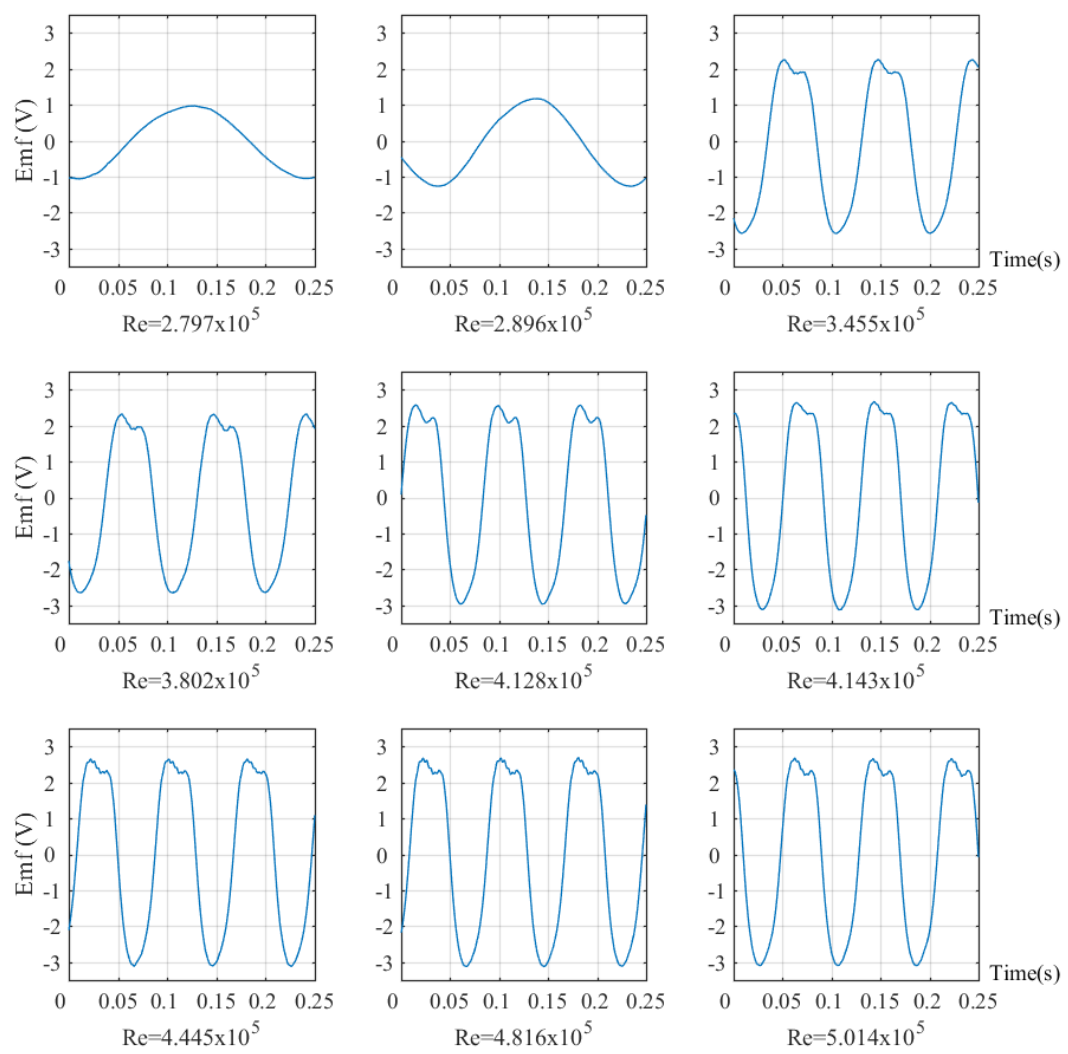

Fig.127 Time evolution of measured emf from harvester B at various Reynolds numbers. 

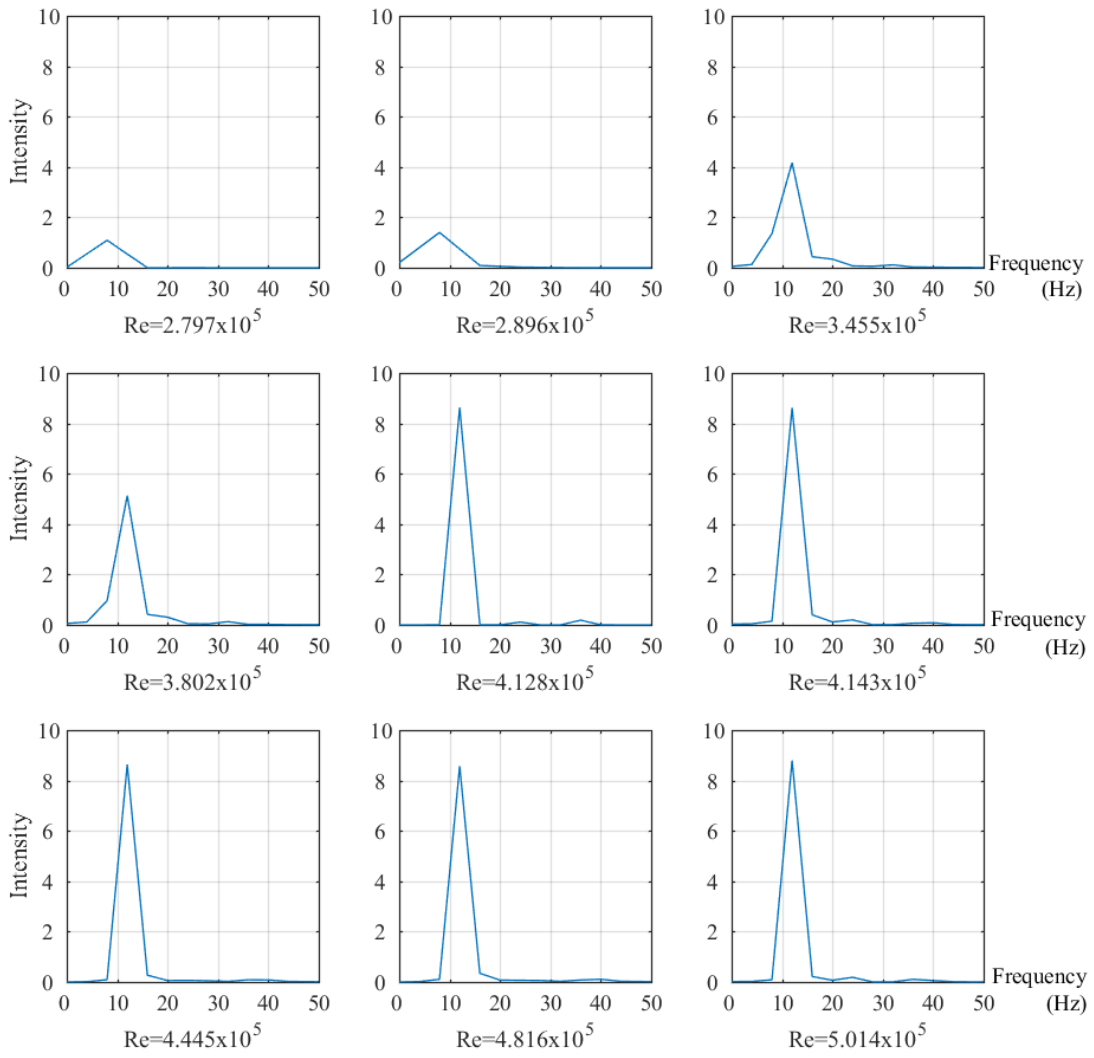

Fig.128 FFT plot of measured emf from harvester $B$ at various Reynolds numbers. 

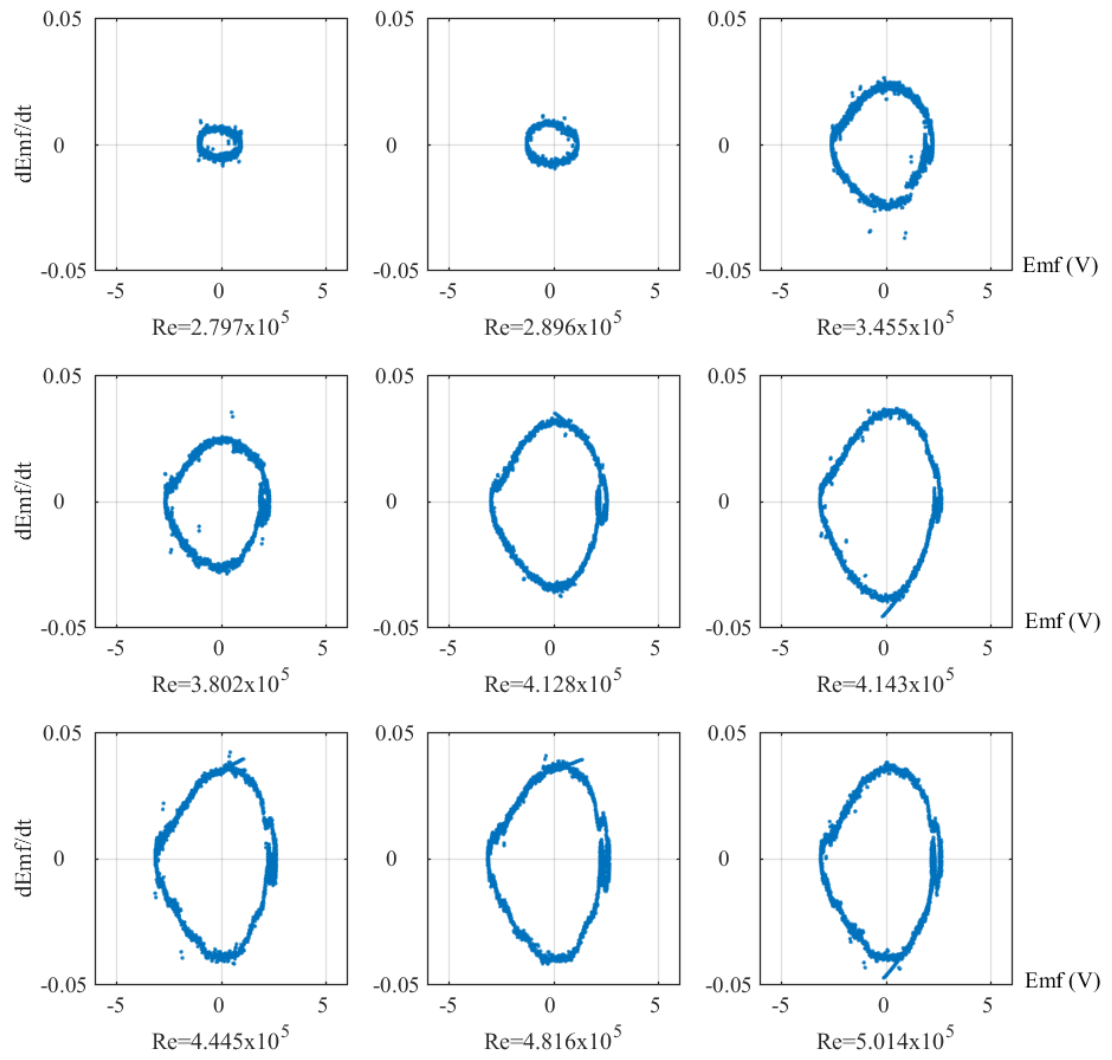

Fig.129 Phase plot of measured emf from harvester B at various Reynolds numbers.

\section{Harvester C}

Table 35 Experimental measurements results of energy harvester C

\begin{tabular}{ccc}
\hline Air flow speed $(\mathrm{m} / \mathrm{s})$ & RPM & Peak emf $(\mathrm{V})$ \\
\hline $\mathbf{7 . 4 6}$ & 174 & 0.718 \\
$\mathbf{8 . 3 3}$ & 367 & 1.316 \\
$\mathbf{8 . 4 5}$ & 442 & 1.752
\end{tabular}




\subsection{9}

9.41

10.15
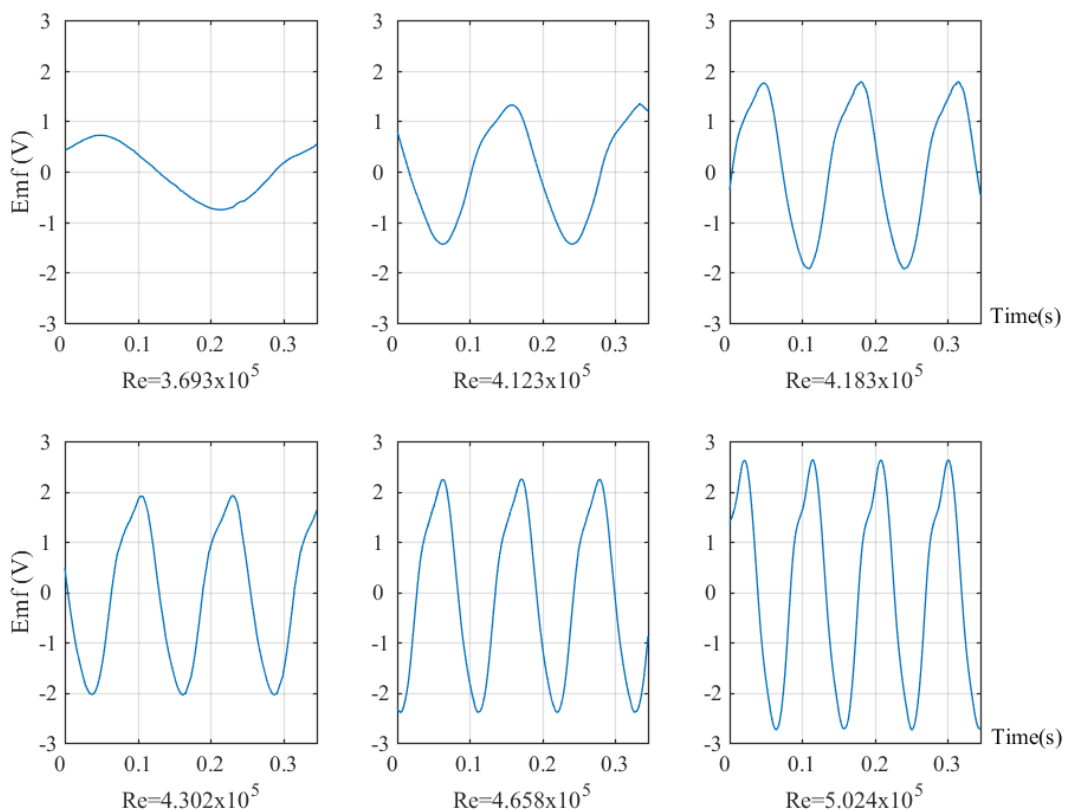

Fig.130 Time evolution of measured emf from harvester $C$ at various Reynolds numbers. 

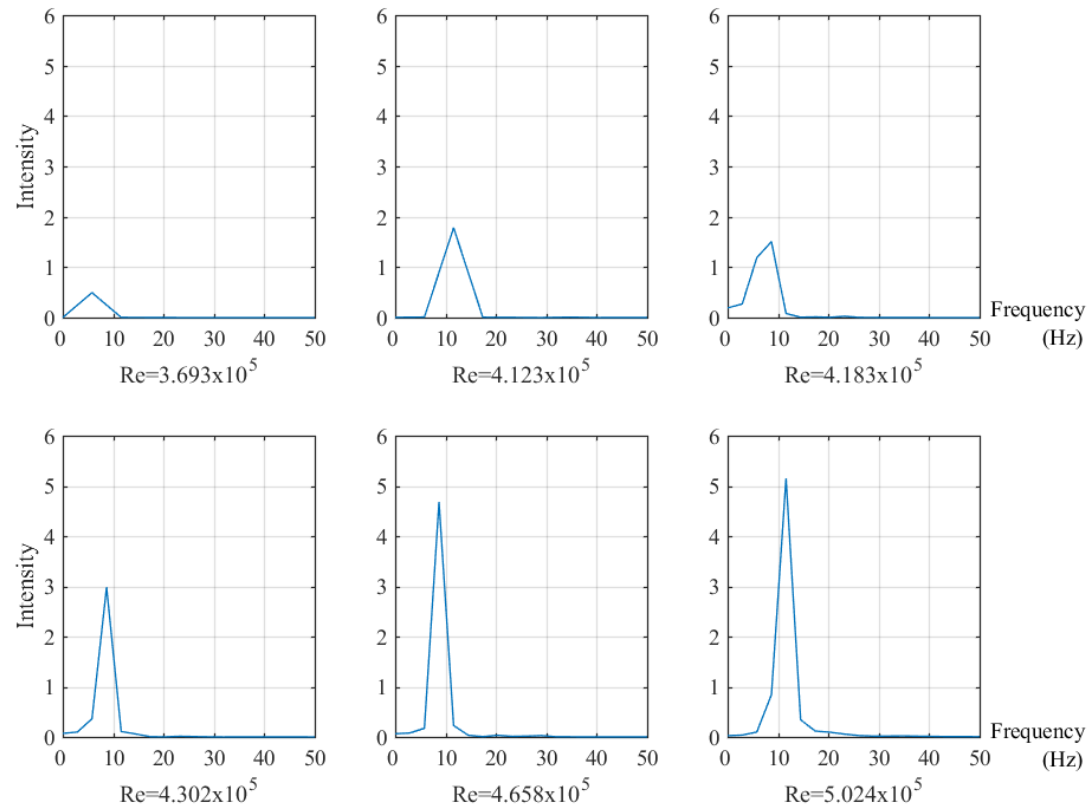

Fig.131 FFT plot of measured emf from harvester $\mathrm{C}$ at various Reynolds numbers.
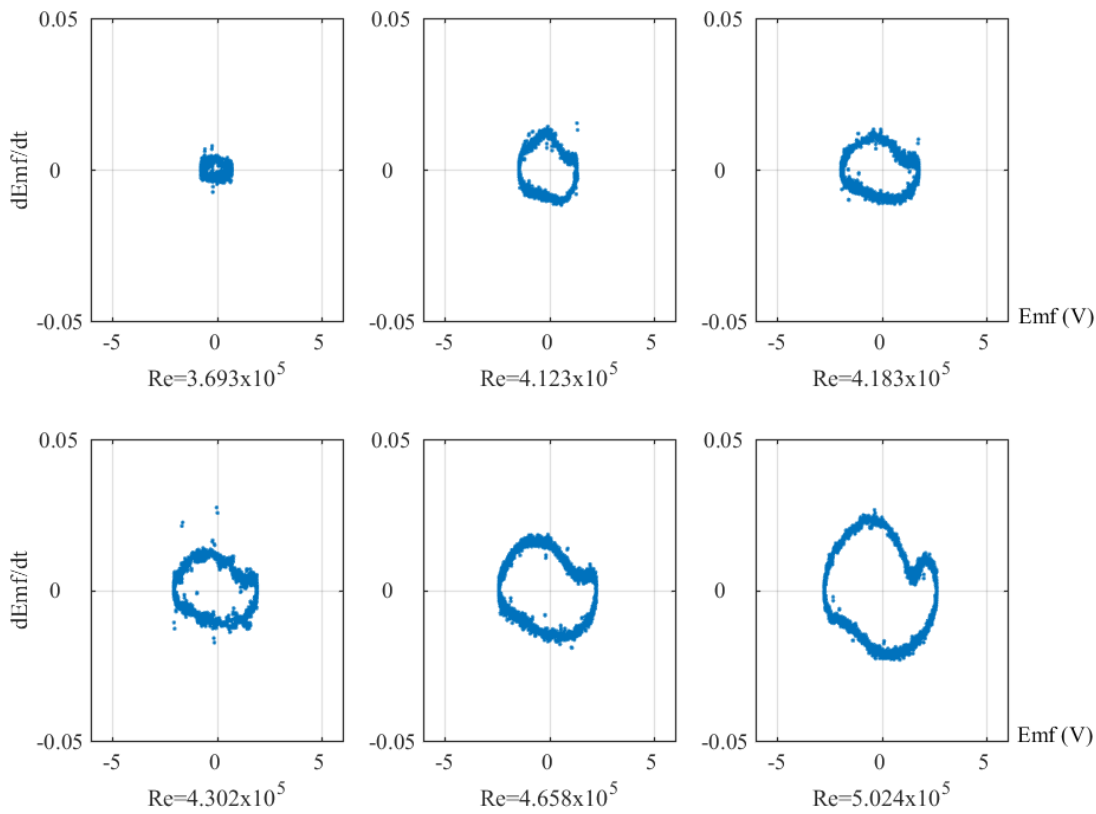

Fig.132 Phase plot of measured emf from harvester $\mathrm{C}$ at various Reynolds numbers.

\section{Harvester D}

Table 36 Experimental measurements results of energy harvester D 


\begin{tabular}{ccc}
\hline Air flow speed $(\mathbf{m} / \mathbf{s})$ & RPM & Peak emf $(\mathrm{V})$ \\
\hline $\mathbf{7 . 2 8}$ & 159 & 0.52 \\
$\mathbf{8 . 2 2}$ & 286 & 1.086 \\
$\mathbf{9 . 0 9}$ & 418 & 1.614 \\
$\mathbf{9 . 4 9}$ & 475 & 1.849 \\
$\mathbf{9 . 9 0}$ & & 2.1 \\
$\mathbf{1 0 . 3 3}$ & 536 & 2.289 \\
\hline
\end{tabular}
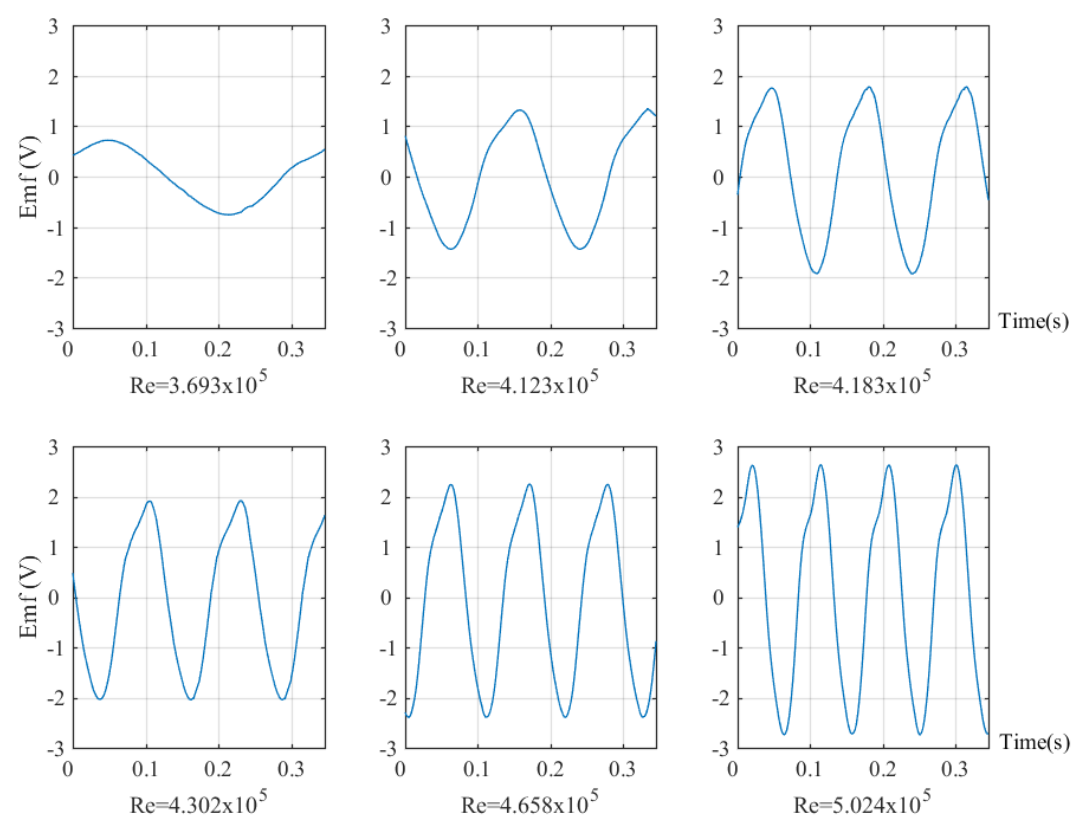

Fig.133 Time evolution of measured emf from harvester D at various Reynolds numbers. 

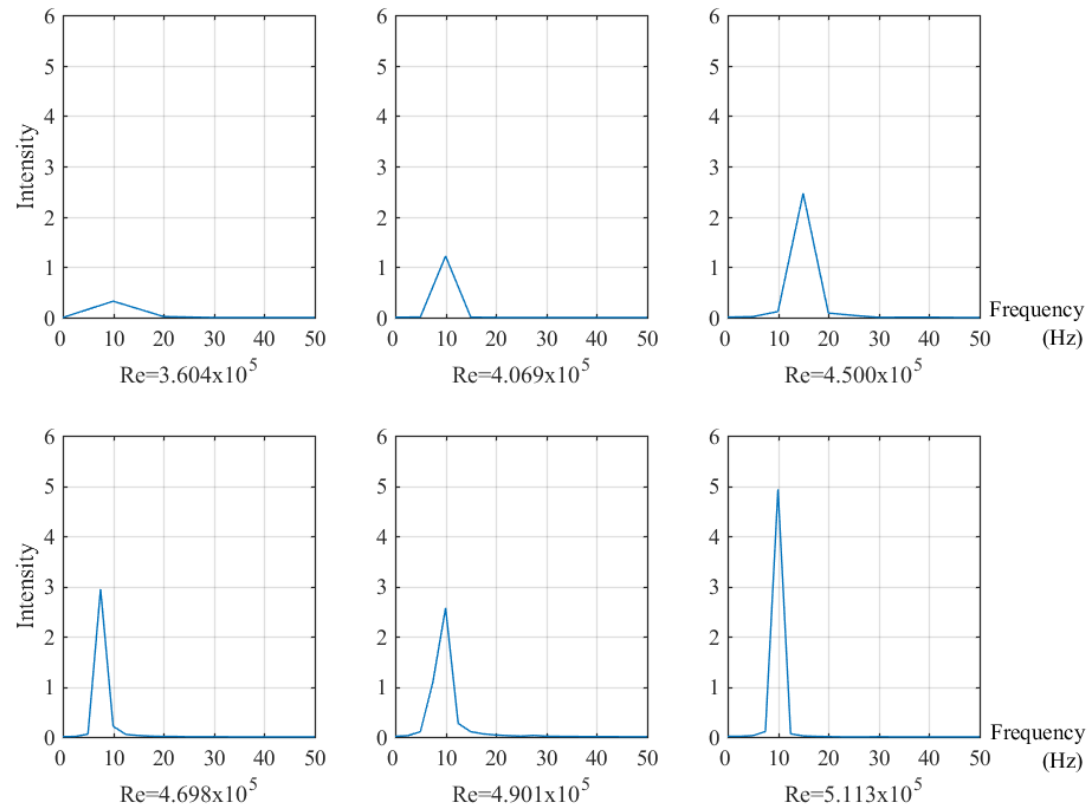

Fig.134 FFT plot of measured emf from harvester D at various Reynolds numbers.
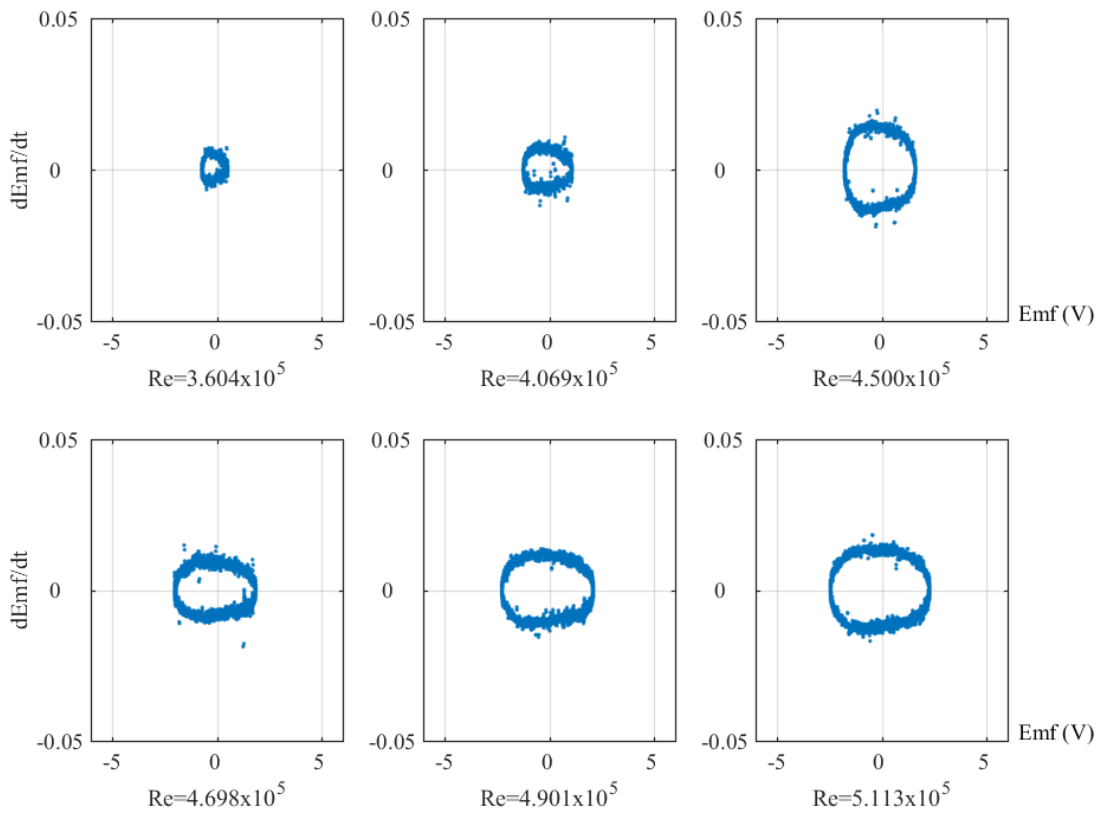

Fig.135 Phase plot of measured emf from harvester D at various Reynolds numbers. 


\section{Harvester E}

Table 37 Experimental measurements results of energy harvester E

\begin{tabular}{|c|c|c|}
\hline Air flow speed $(\mathrm{m} / \mathrm{s})$ & $\mathbf{R P M}$ & Peak emf $(V)$ \\
\hline 3.85 & 118 & 0.457 \\
\hline 4.45 & 155 & 0.628 \\
\hline 5.14 & 329 & 1.035 \\
\hline 5.92 & 474 & 1.840 \\
\hline 6.53 & 582 & 2.394 \\
\hline 7.59 & 692 & 2.806 \\
\hline 8.12 & 748 & 3.060 \\
\hline 9.15 & 877 & 3.614 \\
\hline 9.58 & 897 & 3.663 \\
\hline 10.51 & 942 & 3.891 \\
\hline
\end{tabular}



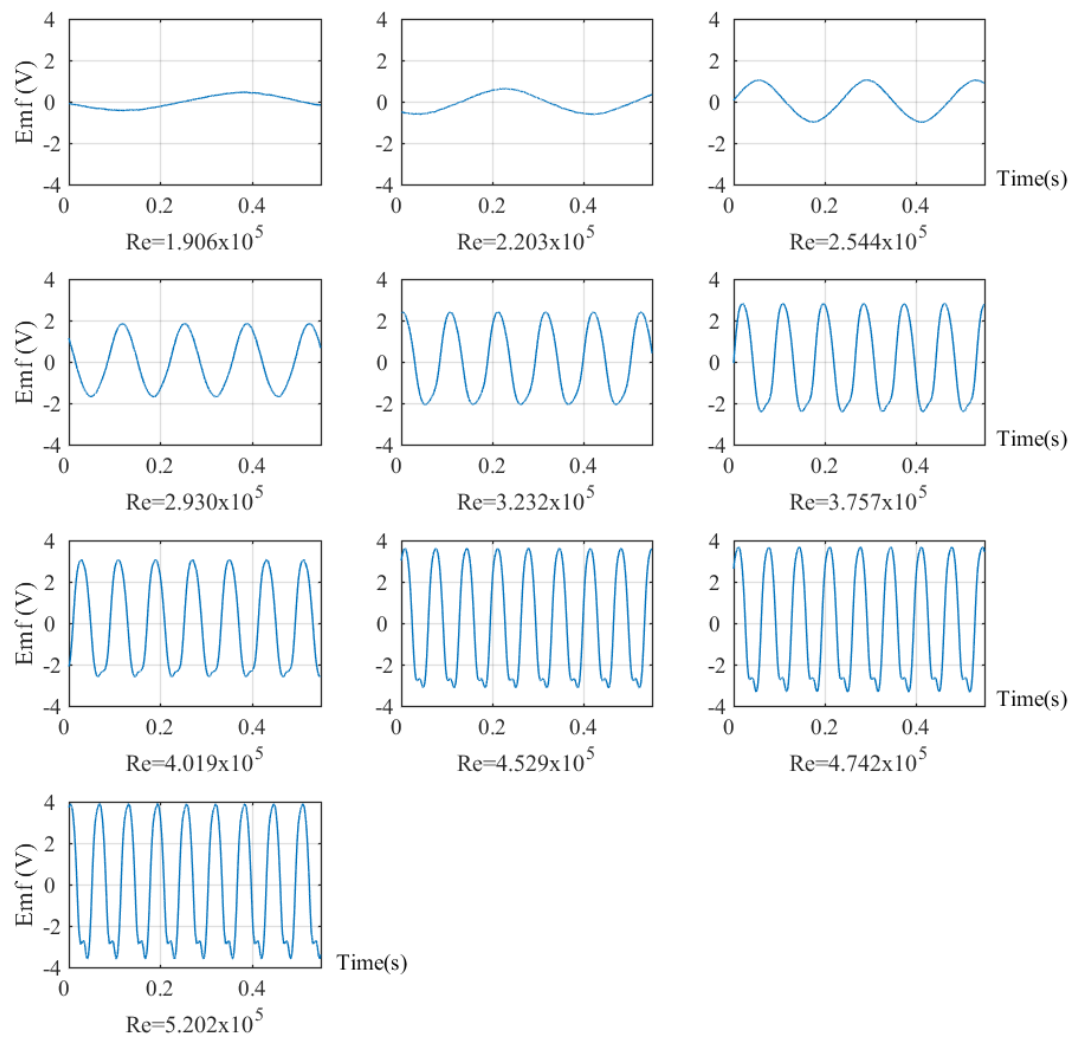

Fig.136 Time evolution of measured emf from harvester $\mathbf{E}$ at various Reynolds numbers. 

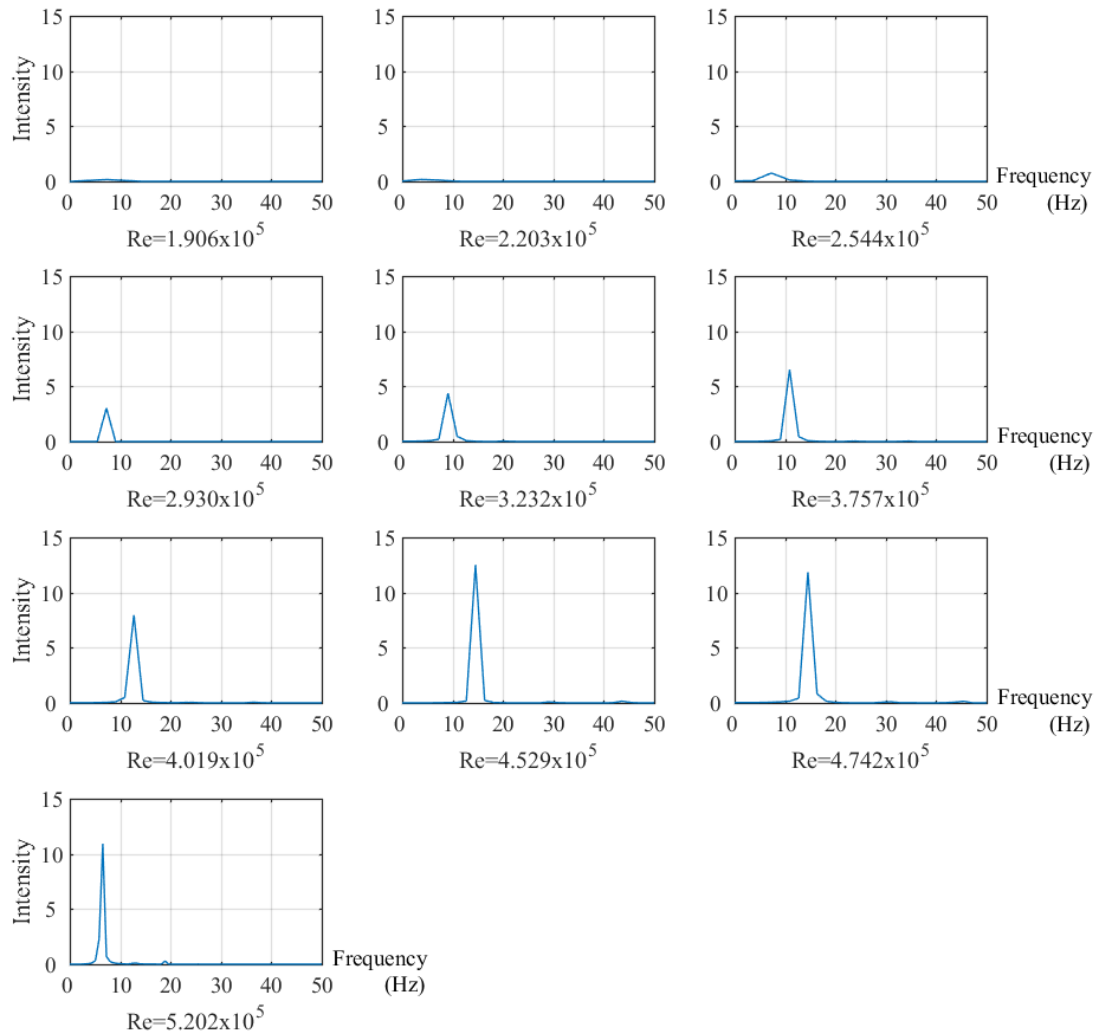

Fig.137 FFT plot of measured emf from harvester $\mathbf{E}$ at various Reynolds numbers. 

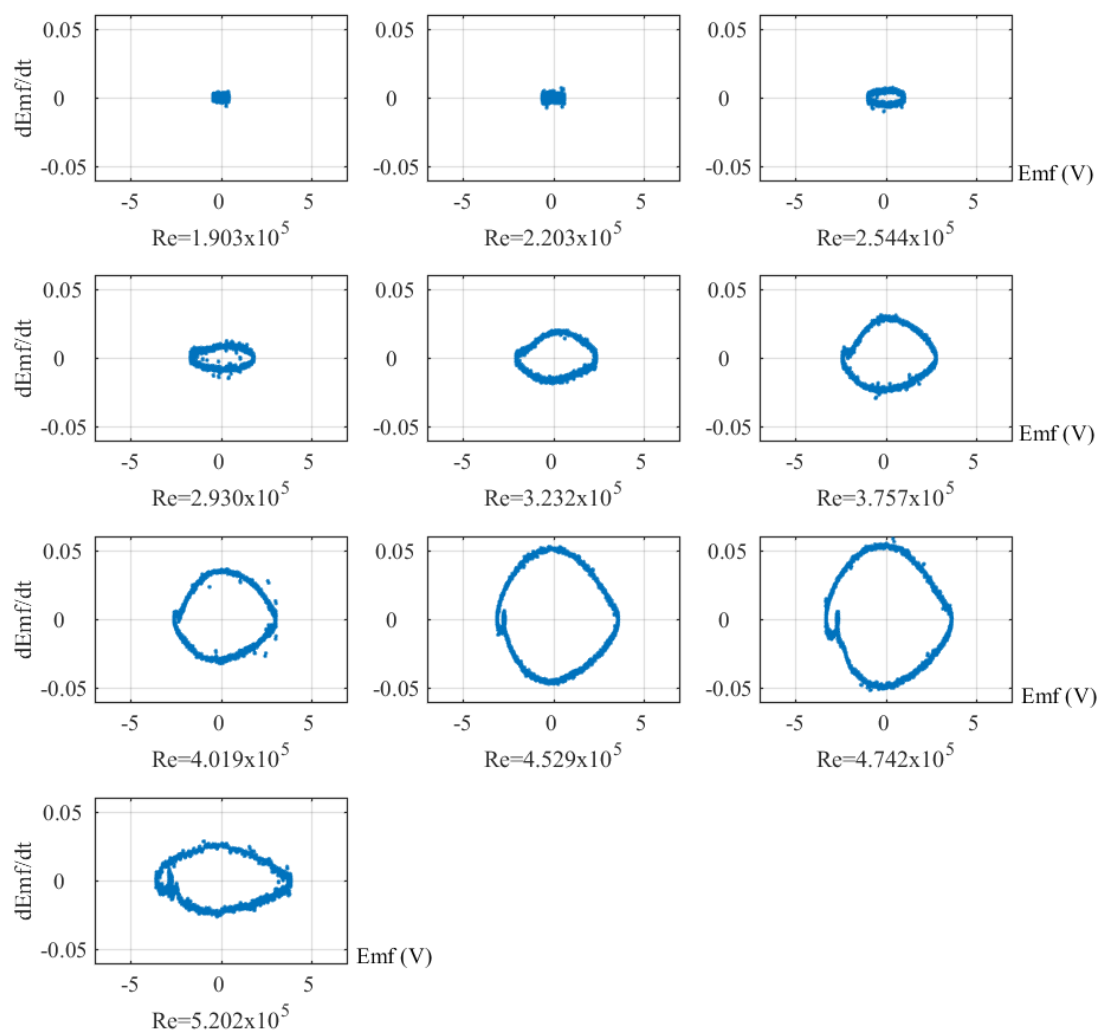

Fig.138 Phase plot of measured emf from harvester $\mathbf{E}$ at various Reynolds numbers.

\section{Harvester F}

Table 38 Experimental measurements results of energy harvester $\mathbf{F}$

\begin{tabular}{ccc}
\hline Air flow speed $(\mathbf{m} / \mathbf{s})$ & RPM & Peak emf $(\mathbf{V})$ \\
\hline $\mathbf{5 . 9 3}$ & 51 & 0.189 \\
$\mathbf{6 . 3 3}$ & 84 & 0.314 \\
$\mathbf{7 . 1 4}$ & 159 & 0.633
\end{tabular}



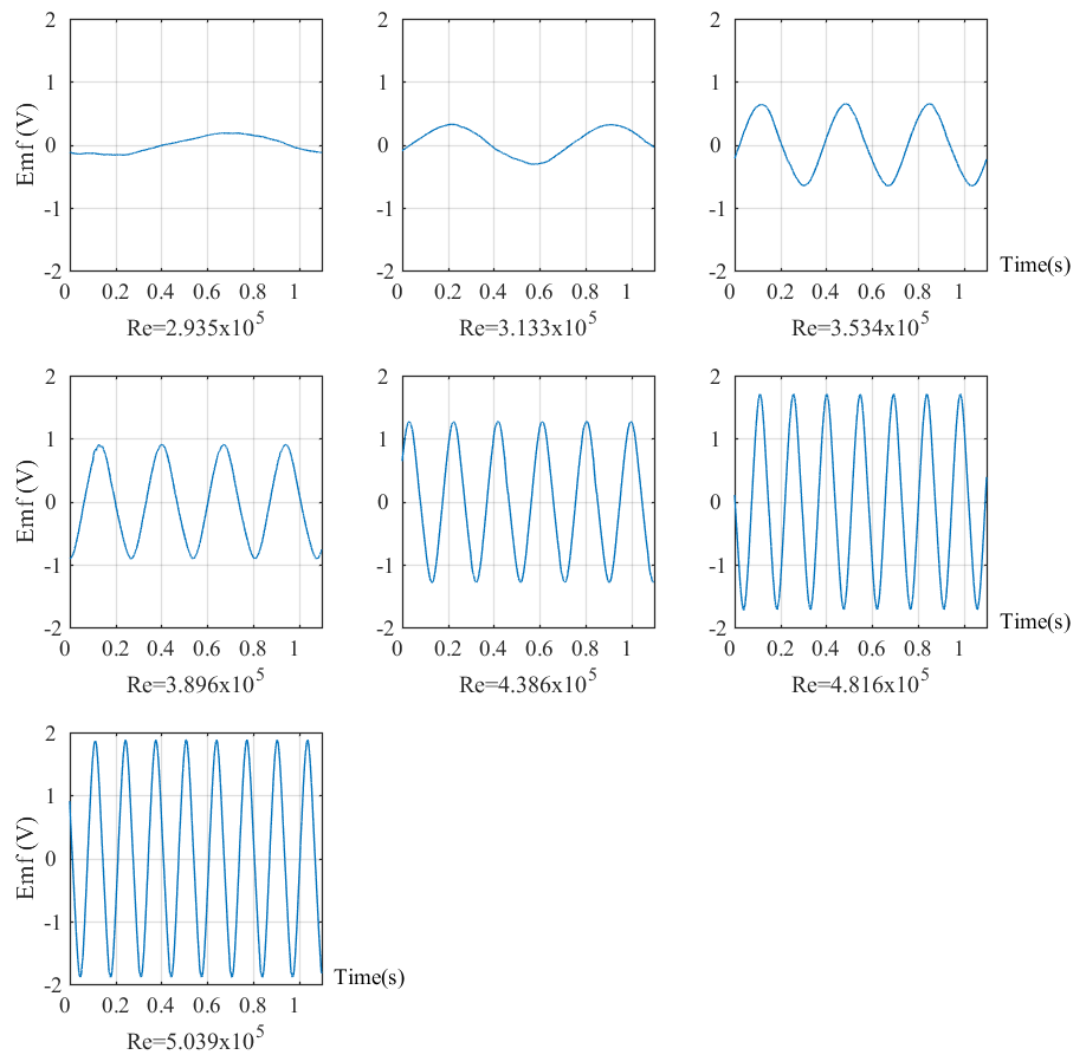

Fig.139 Time evolution of measured emf from harvester $F$ at various Reynolds numbers. 

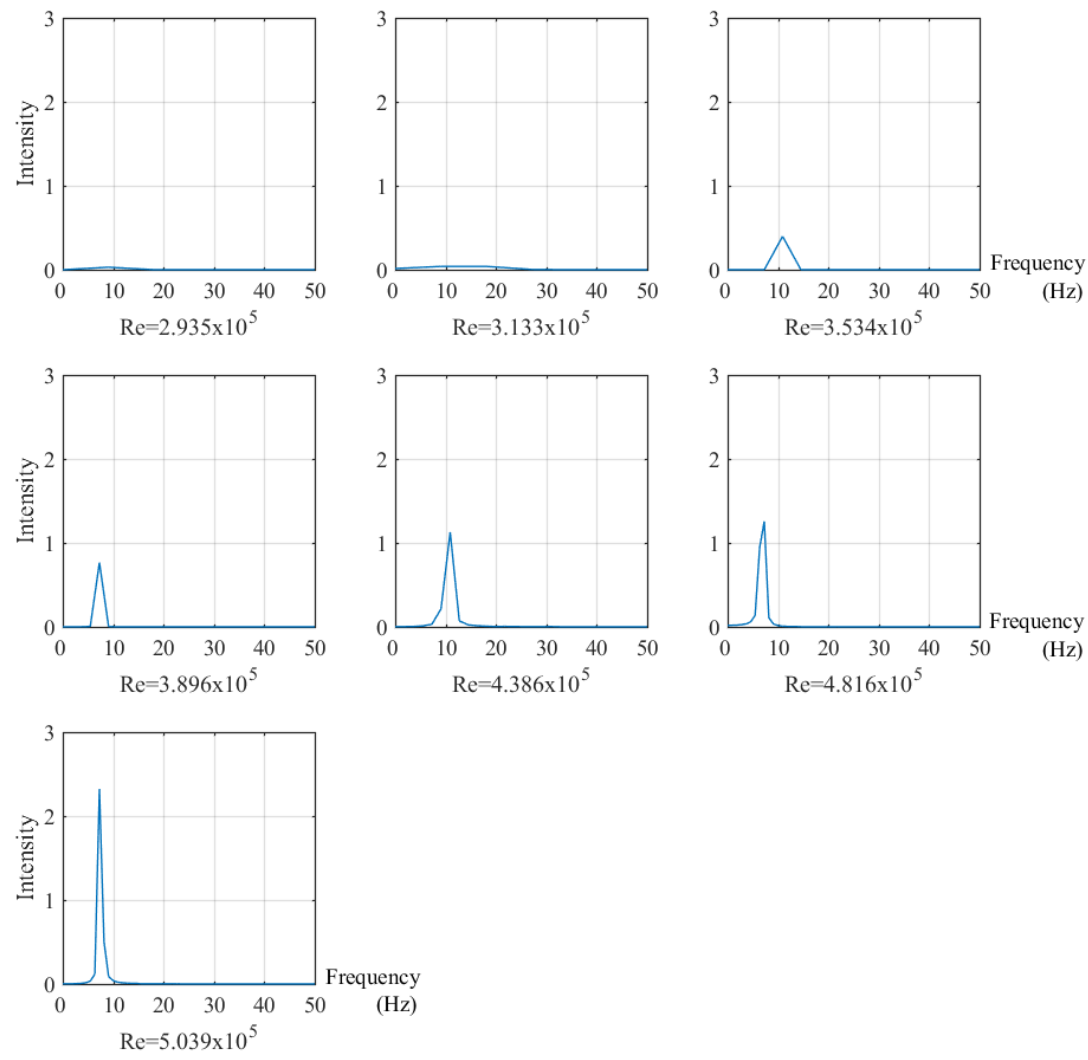

Fig.140 FFT plot of measured emf from harvester $F$ at various Reynolds numbers. 

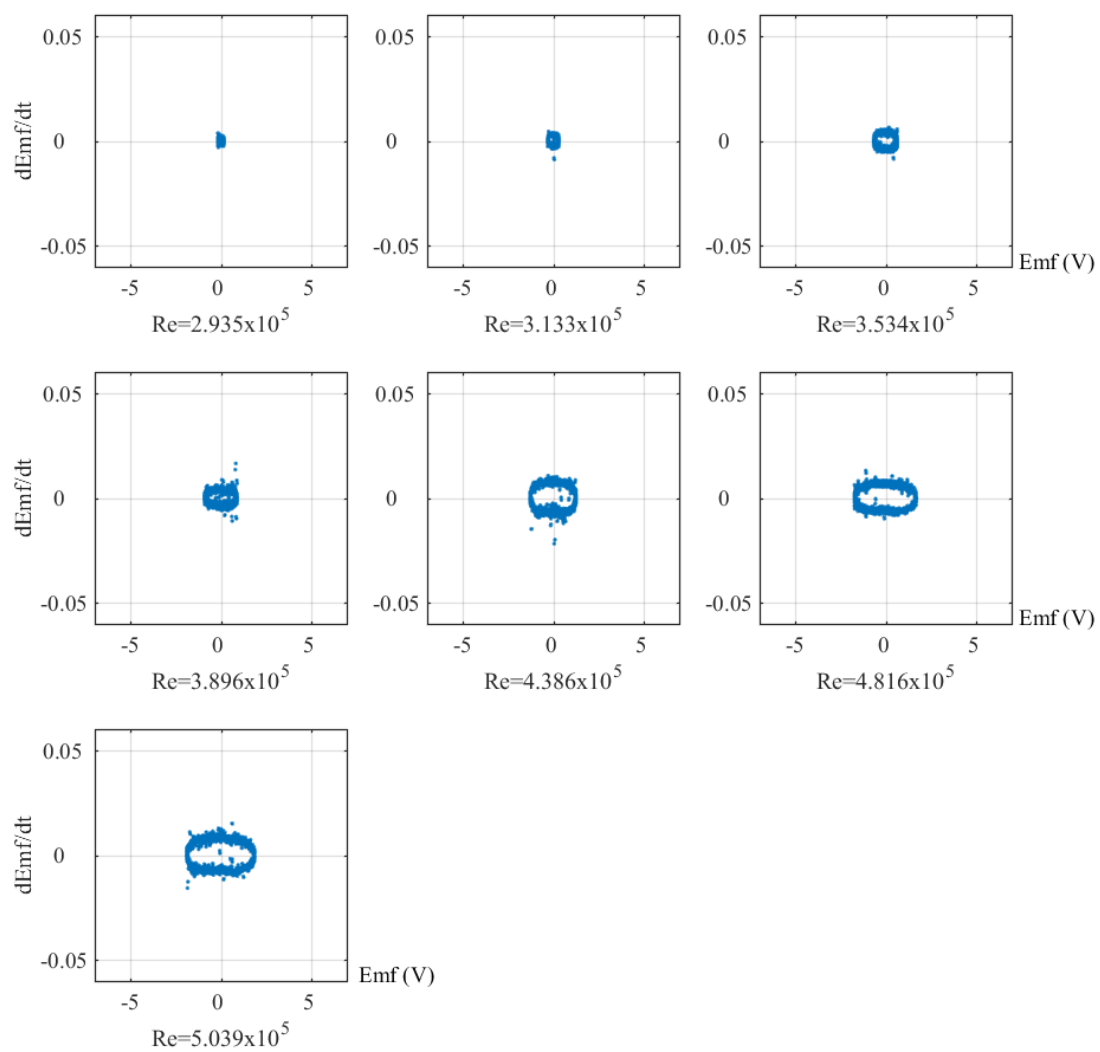

Fig.141 Phase plot of measured emf from harvester $\mathrm{F}$ at various Reynolds numbers.

\section{Harvester G}

Table 39 Experimental measurements results of energy harvester G

\begin{tabular}{ccc}
\hline Air flow speed $(\mathbf{m} / \mathbf{s})$ & RPM & Peak emf $(\mathbf{V})$ \\
\hline $\mathbf{7 . 6 6}$ & 323 & 1.281 \\
$\mathbf{7 . 8 7}$ & 351 & 1.399 \\
$\mathbf{8 . 5 6}$ & 451 & 1.809
\end{tabular}


9.18

9.38

10.13
497

607

740
2.004

2.431

2.991
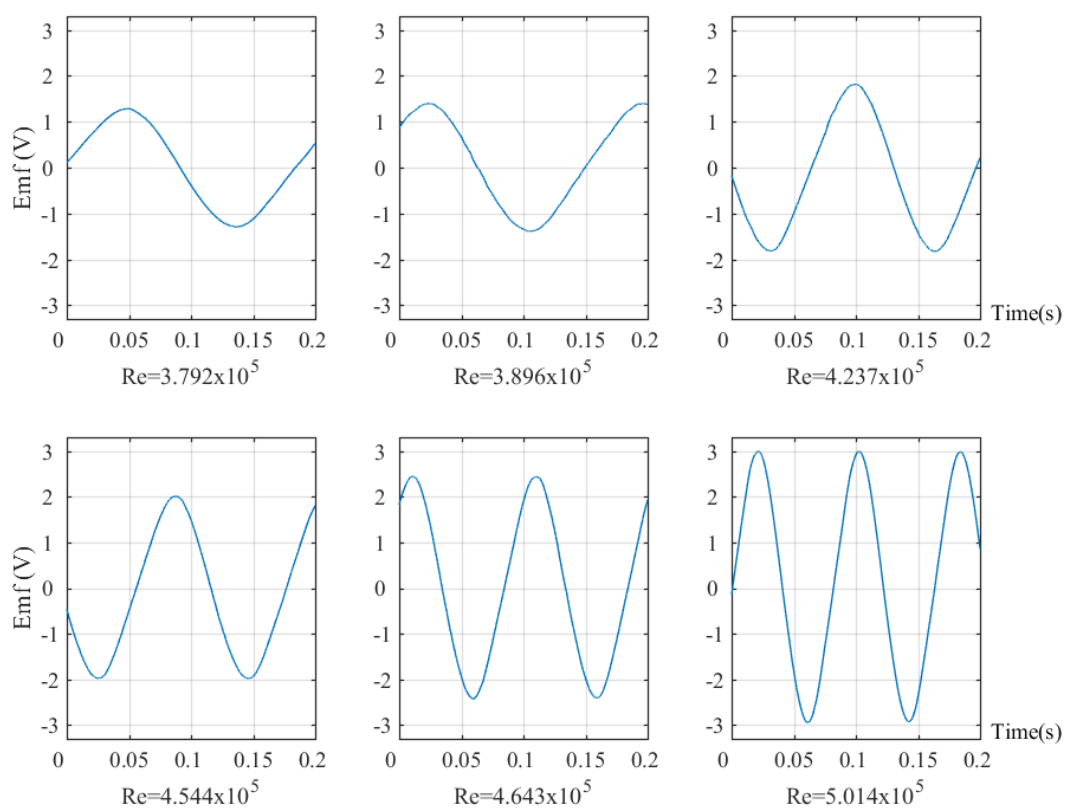

Fig.142 Time evolution of measured emf from harvester $G$ at various Reynolds numbers. 

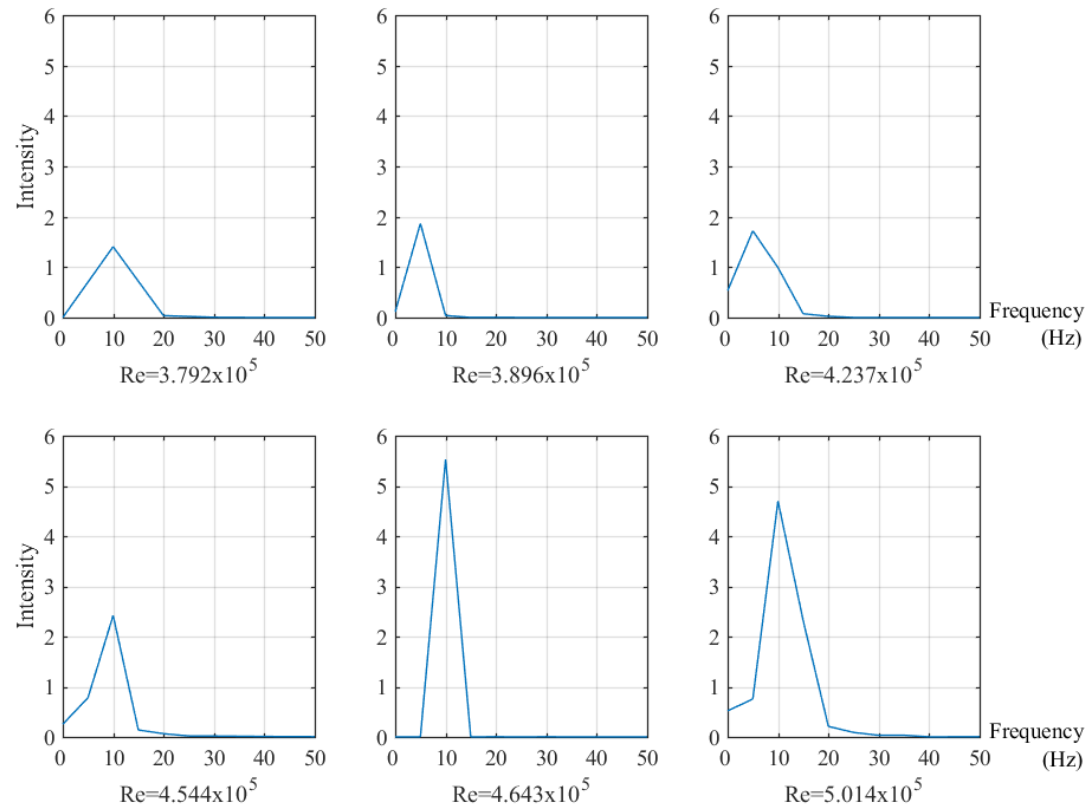

Fig.143 FFT plot of measured emf from harvester $G$ at various Reynolds numbers.
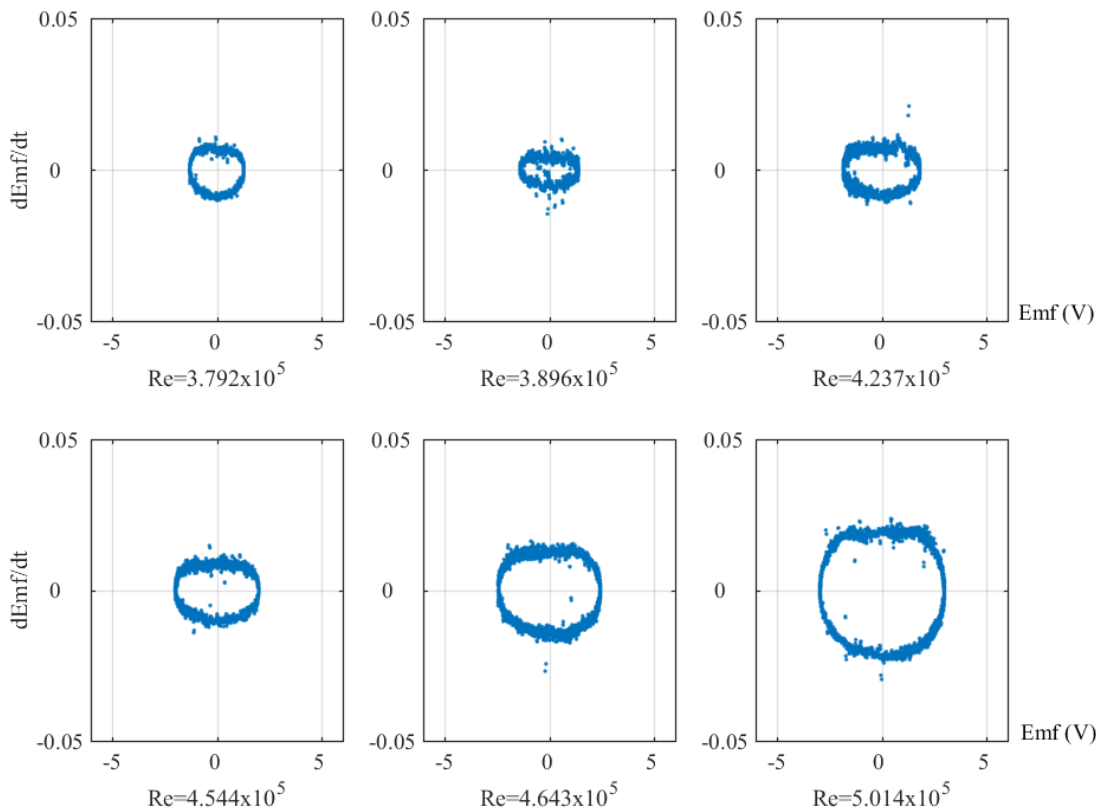

Fig.144 Phase plot of measured emf from harvester $G$ at various Reynolds numbers. 


\section{Harvester H}

Table 40 Experimental measurements results of energy harvester $\mathbf{H}$

\begin{tabular}{ccc}
\hline Air flow speed $(\mathrm{m} / \mathrm{s})$ & RPM & Peak emf $(\mathrm{V})$ \\
\hline $\mathbf{5 . 4 5}$ & 98 & 0.406 \\
$\mathbf{6 . 1 9}$ & 153 & 0.586 \\
$\mathbf{6 . 8 6}$ & 268 & 1.109 \\
$\mathbf{7 . 7 6}$ & 499 & 2.202 \\
$\mathbf{8 . 0 1}$ & 519 & 2.315 \\
$\mathbf{8 . 9 9}$ & 571 & 2.541 \\
$\mathbf{9 . 8 7}$ & 579 & 2.561 \\
$\mathbf{1 0 . 2 3}$ & 569 & 2.518 \\
\hline
\end{tabular}



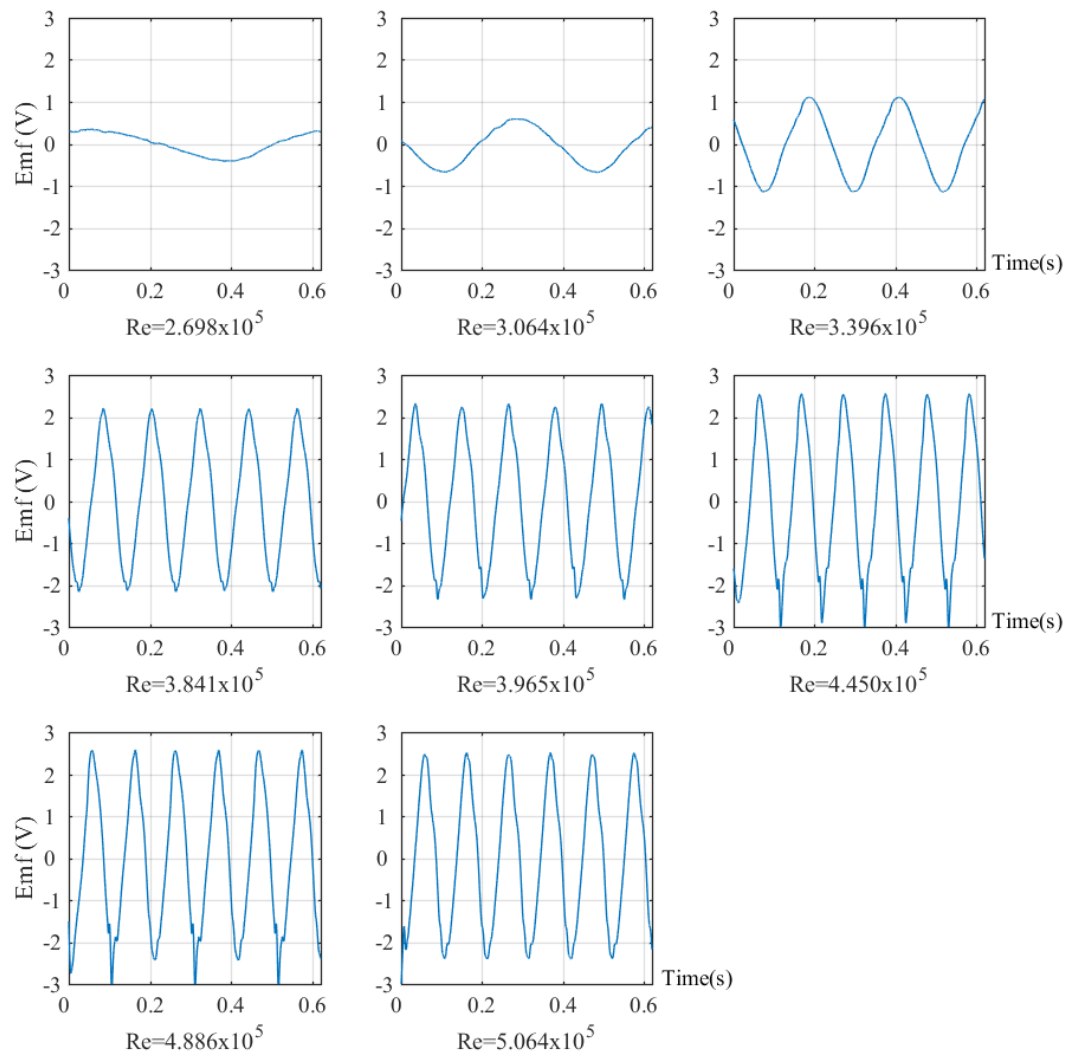

Fig.145 Time evolution of measured emf from harvester $H$ at various Reynolds numbers. 

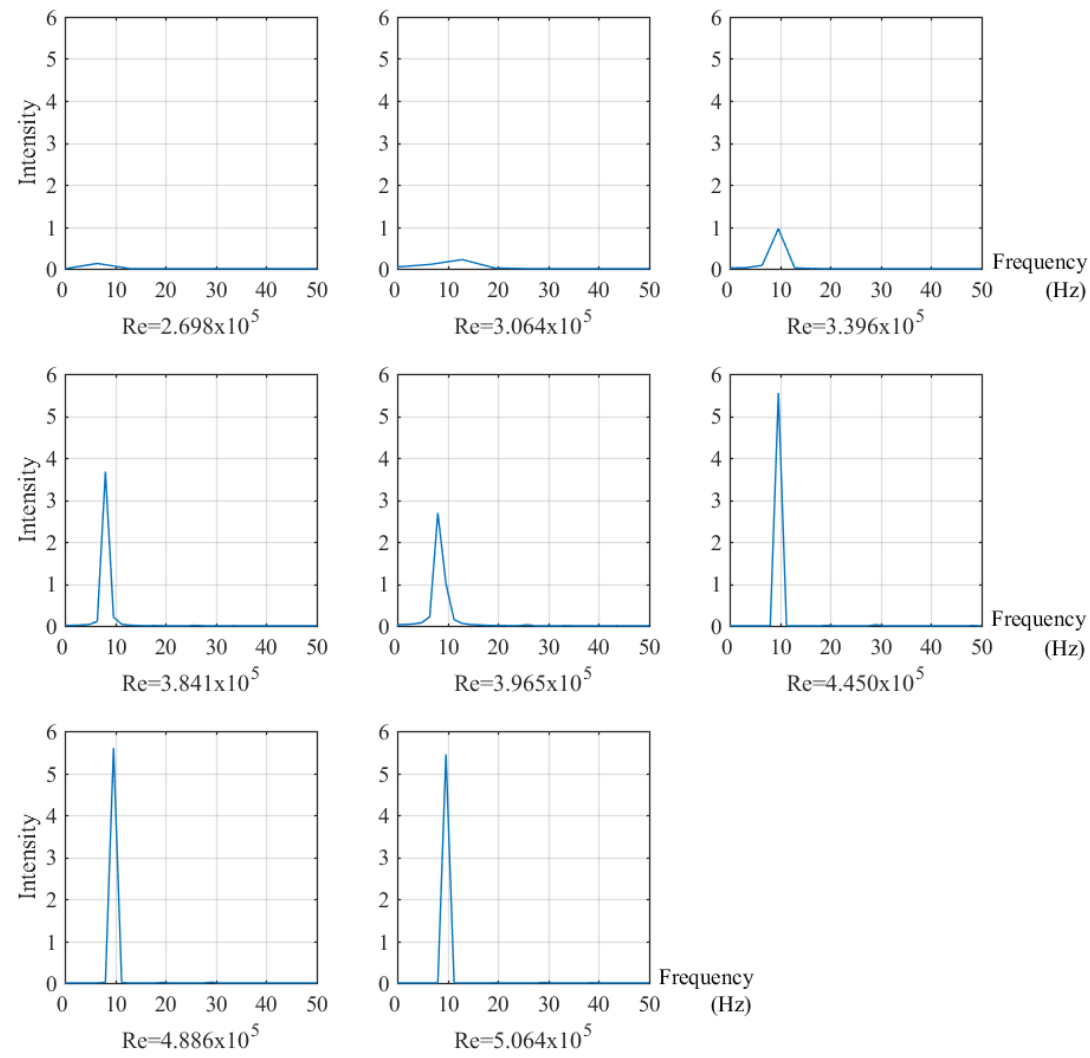

Fig.146 FFT plot of measured emf from harvester $H$ at various Reynolds numbers. 

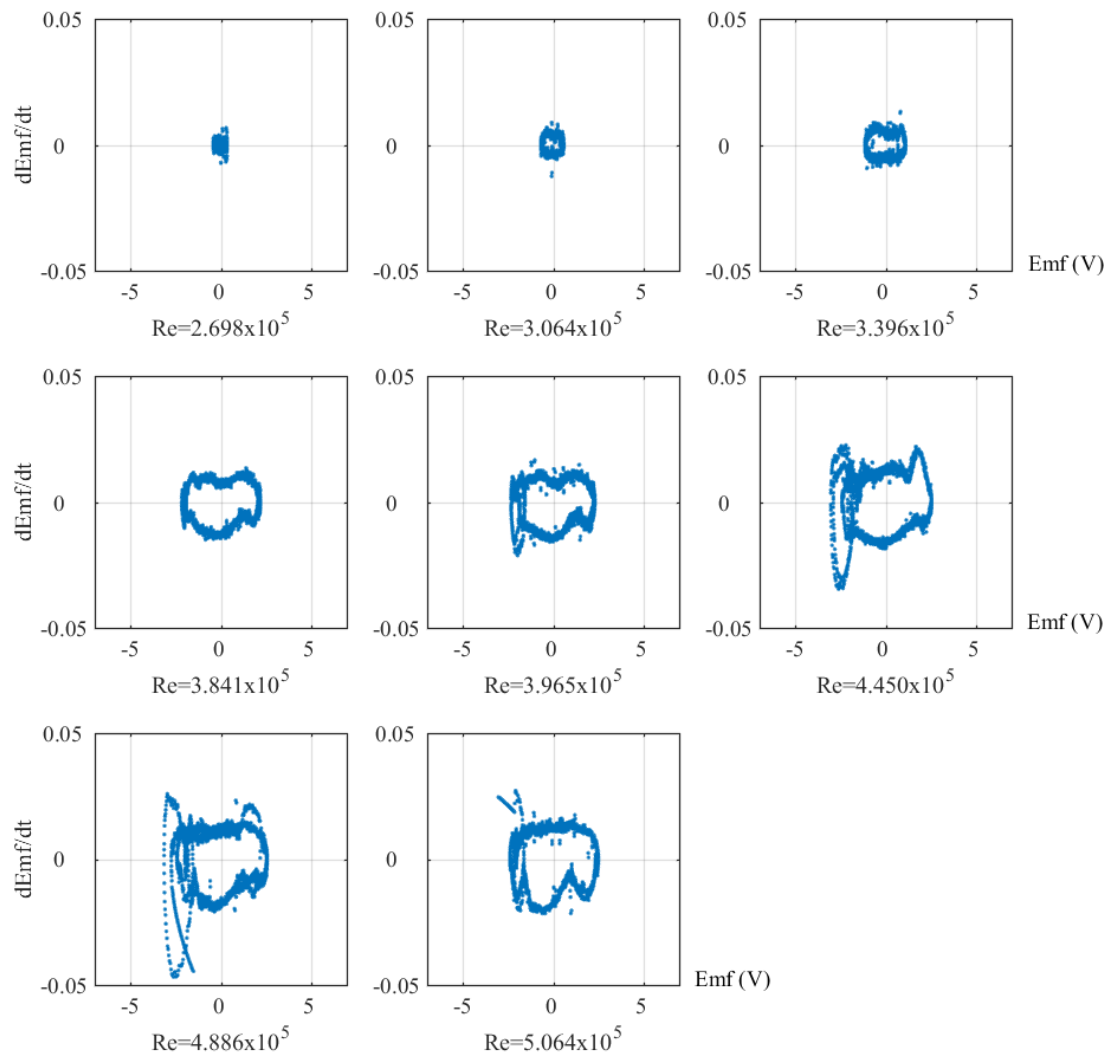

Fig.147 Phase plot of measured emf from harvester $\mathrm{H}$ at various Reynolds numbers.

\section{Harvester I}

Table 41 Experimental measurements results of energy harvester I

\begin{tabular}{ccc}
\hline Air flow speed $(\mathrm{m} / \mathrm{s})$ & RPM & Peak emf $(\mathrm{V})$ \\
\hline $\mathbf{6 . 0 6}$ & 93 & 0.319 \\
$\mathbf{6 . 6 5}$ & 154 & 0.604 \\
$\mathbf{7 . 2 3}$ & 234 & 0.936
\end{tabular}


8.37

8.88

9.53

10.19
363

404

452

546
1.401

1.640

1.835

2.224
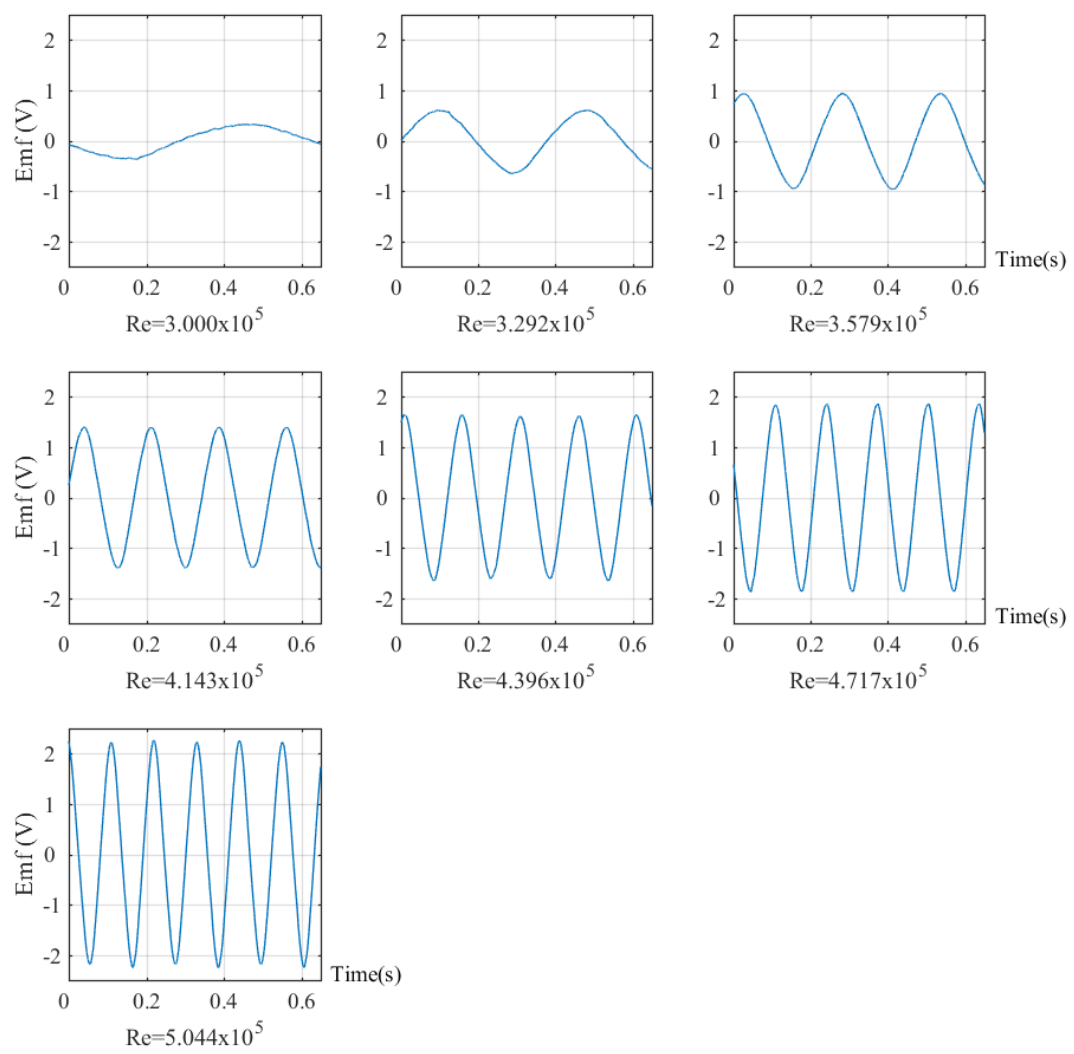

Fig.148 Time evolution of measured emf from harvester I at various Reynolds numbers. 

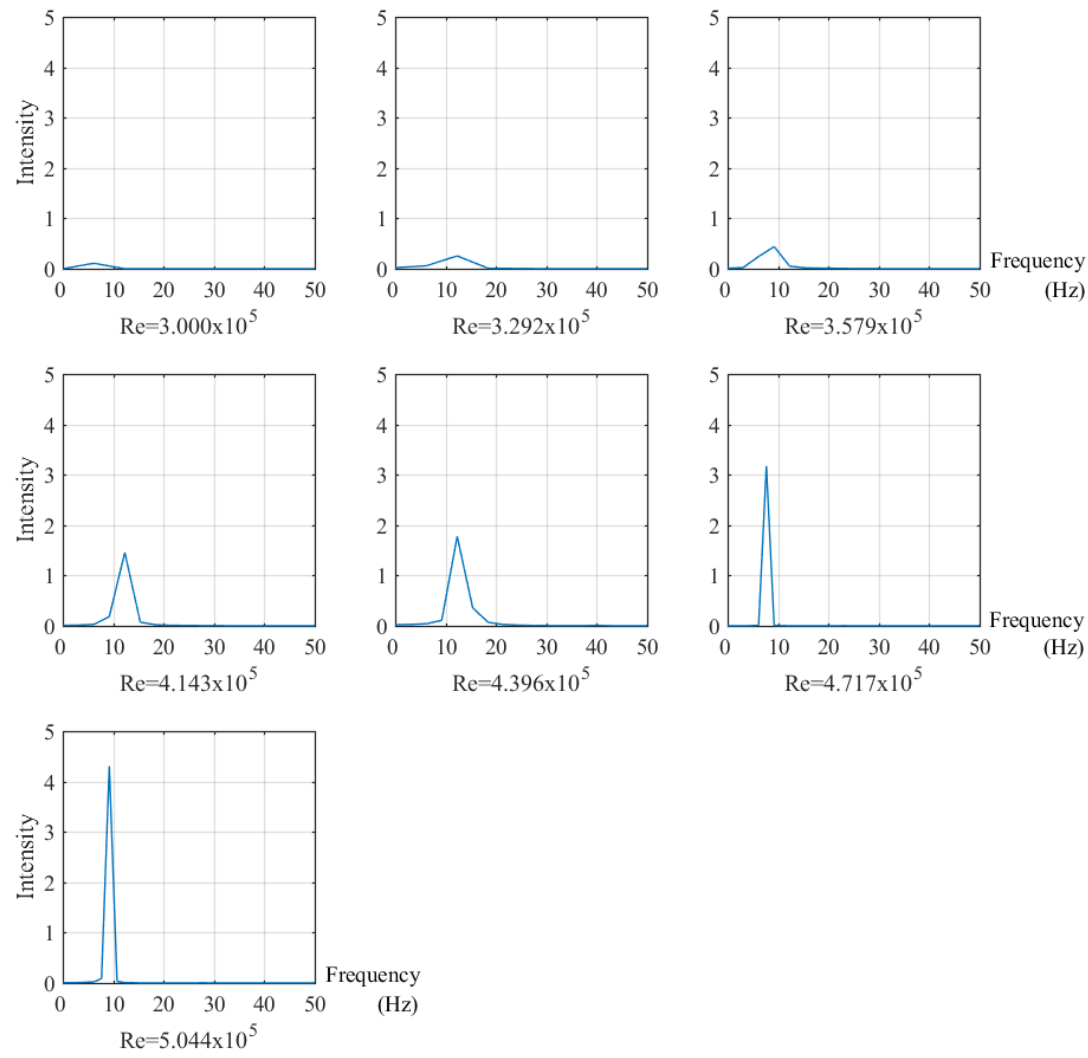

Fig.149 FFT plot of measured emf from harvester I at various Reynolds numbers. 

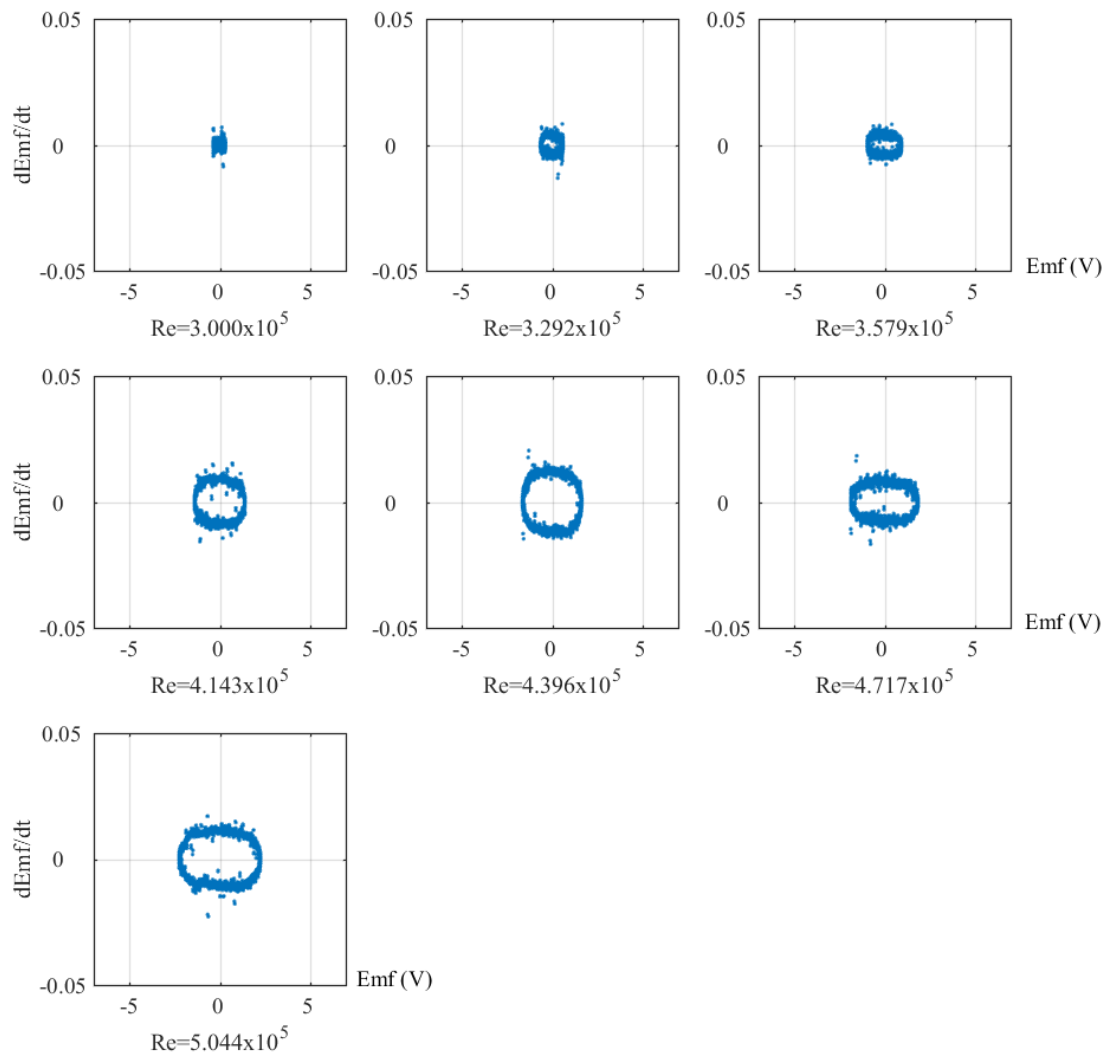

Fig.150 Phase plot of measured emf from harvester I at various Reynolds numbers.

\section{Harvester J}

Table 42 Experimental measurements results of energy harvester J

\begin{tabular}{ccc}
\hline Air flow speed $(\mathrm{m} / \mathrm{s})$ & RPM & Peak emf $(\mathrm{V})$ \\
\hline $\mathbf{4 . 0 4}$ & 194 & 0.369 \\
$\mathbf{4 . 5 1}$ & 260 & 0.601 \\
$\mathbf{4 . 9 5}$ & 505 & 1.609
\end{tabular}




\begin{tabular}{lll}
$\mathbf{5 . 2 5}$ & 586 & 2.019 \\
$\mathbf{5 . 8 1}$ & 720 & 2.591 \\
$\mathbf{6 . 6 5}$ & 885 & 3.354 \\
$\mathbf{7 . 3 0}$ & 962 & 3.768 \\
$\mathbf{8 . 2 4}$ & 1123 & 4.339 \\
$\mathbf{8 . 4 0}$ & 1173 & 4.619 \\
$\mathbf{9 . 0 2}$ & 1253 & 5.026 \\
$\mathbf{9 . 6 3}$ & 1309 & 5.224 \\
$\mathbf{1 0 . 3 1}$ & 1344 & 5.378 \\
\hline & & \\
\hline
\end{tabular}



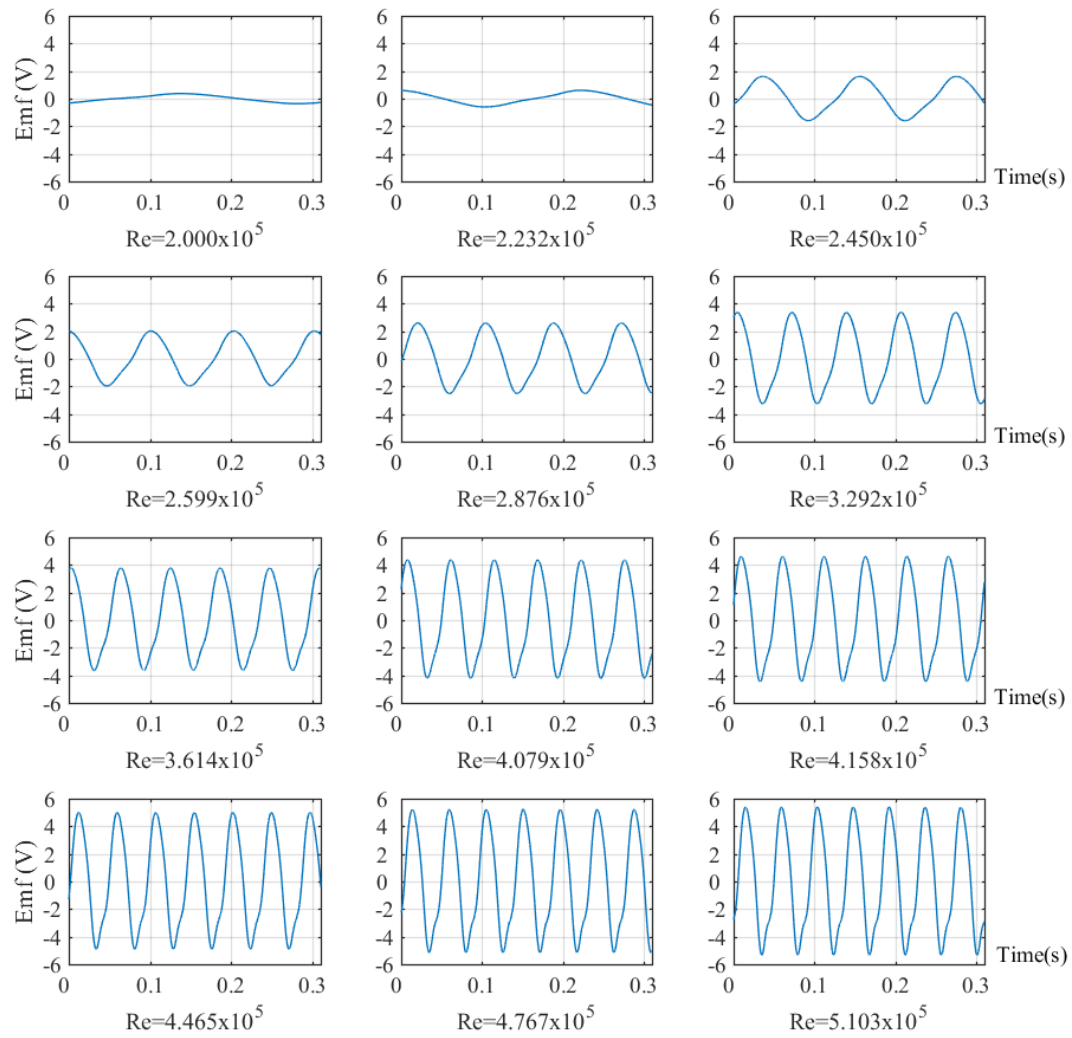

Fig.151 Time evolution of measured emf from harvester $J$ at various Reynolds numbers. 

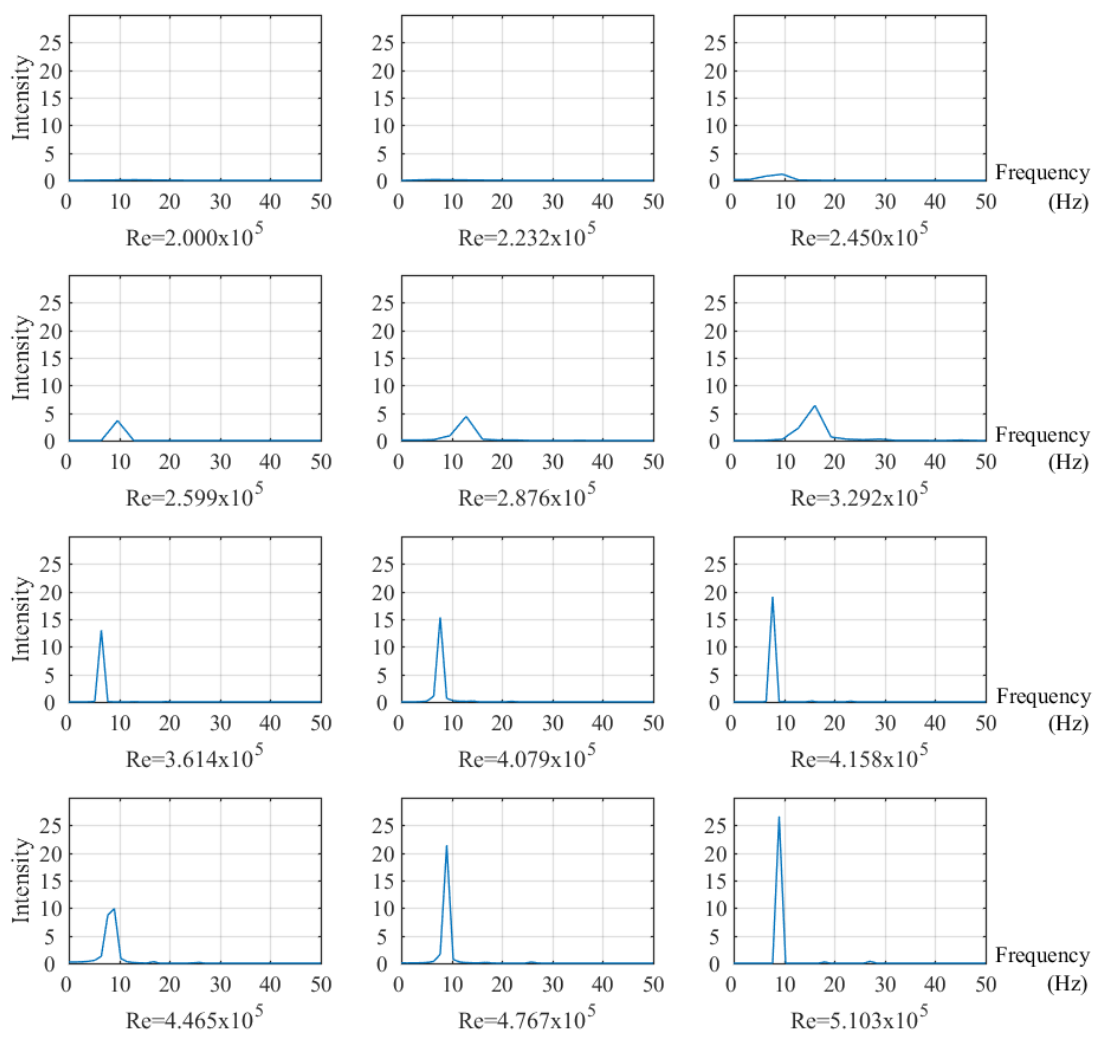

Fig.152 FFT plot of measured emf from harvester $\mathbf{J}$ at various Reynolds numbers. 

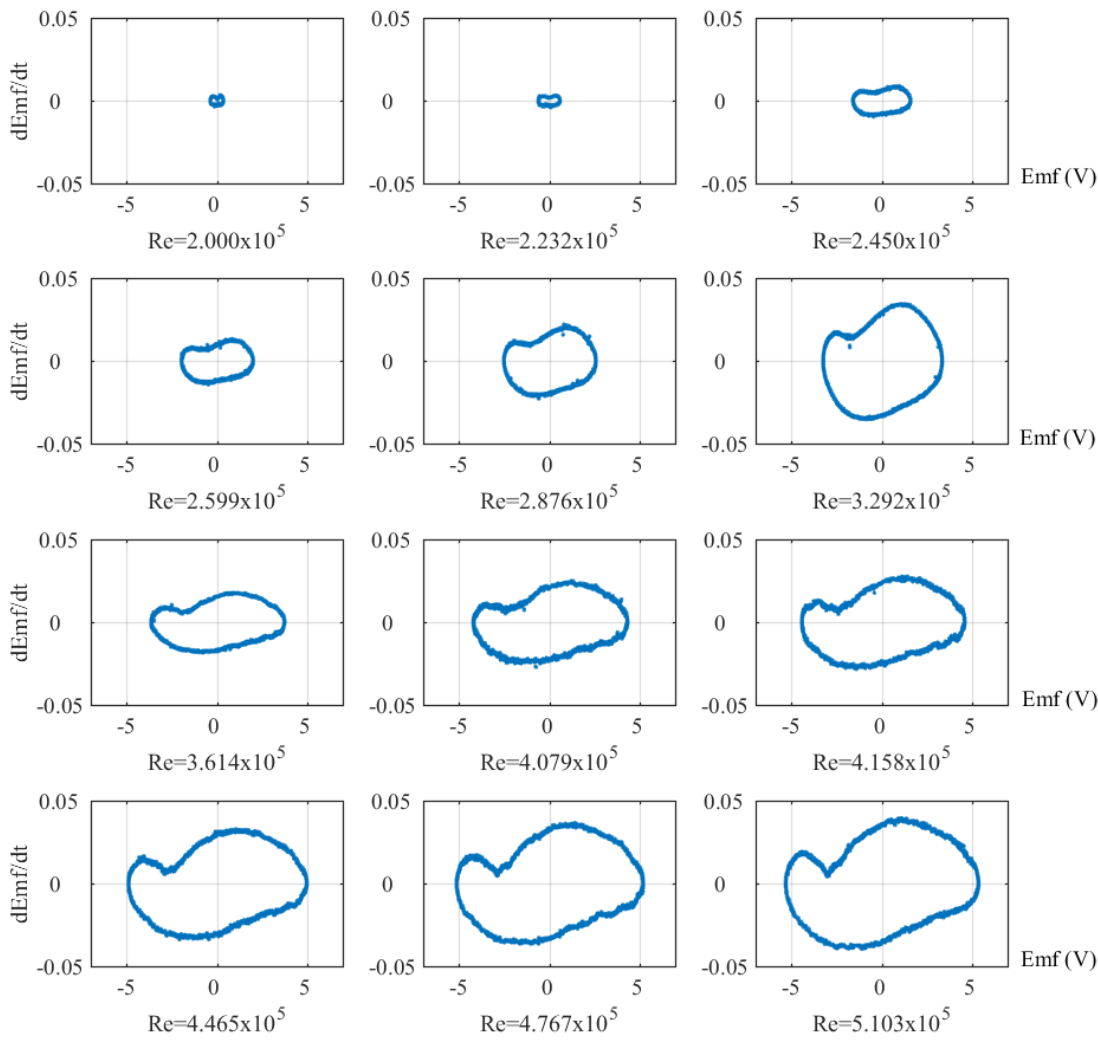

Fig.153 Phase plot of measured emf from harvester $\mathbf{J}$ at various Reynolds numbers. 


\section{Appendix C: Wind tunnel results for harvester orientation case study}

\section{Harvester B ('Anti-clockwise' orientation)}

Table 43 Experimental measurements results of energy harvester B in 'anti-clockwise' orientation

\begin{tabular}{ccc}
\hline Air flow speed $(\mathbf{m} / \mathbf{s})$ & RPM & Peak emf $(\mathbf{V})$ \\
\hline $\mathbf{4 . 6 0}$ & 134 & 0.158 \\
$\mathbf{5 . 6 5}$ & 434 & 1.34 \\
$\mathbf{6 . 3 4}$ & 653 & 2.16 \\
$\mathbf{7 . 1 1}$ & 850 & 2.96 \\
$\mathbf{7 . 6 0}$ & 956 & 3.40 \\
$\mathbf{8 . 3 2}$ & 1095 & 3.92 \\
$\mathbf{9 . 0 8}$ & 1154 & 4.24 \\
$\mathbf{9 . 6 1}$ & 1137 & 4.16 \\
$\mathbf{1 0 . 3 4}$ & 1179 & 4.24 \\
\hline
\end{tabular}

\section{Harvester C ('Anti-clockwise' orientation)}

Table 44 Experimental measurements results of energy harvester C in 'anti-clockwise' orientation 


\begin{tabular}{ccc}
\hline Air flow speed $(\mathbf{m} / \mathbf{s})$ & RPM & Peak emf $(\mathbf{V})$ \\
\hline $\mathbf{7 . 9 8}$ & 82 & 0.057 \\
$\mathbf{9 . 0 9}$ & 215 & 0.375 \\
$\mathbf{9 . 8 4}$ & 329 & 0.768 \\
$\mathbf{1 0 . 3 2}$ & 467 & 1.322 \\
\hline
\end{tabular}

\section{Harvester D ('Anti-clockwise' orientation)}

Table 45 Experimental measurements results of energy harvester D in 'anti-clockwise' orientation

\begin{tabular}{ccc}
\hline Air flow speed $(\mathbf{m} / \mathbf{s})$ & RPM & Peak emf $(\mathbf{V})$ \\
\hline $\mathbf{9 . 6 7}$ & 122 & 0.147 \\
$\mathbf{1 0 . 6 8}$ & 260 & 0.522 \\
$\mathbf{1 0 . 9 3}$ & 329 & 0.776 \\
\hline
\end{tabular}

\section{Harvester E ('Anti-clockwise' orientation)}

Table 46 Experimental measurements results of energy harvester E in 'anti-clockwise' orientation

\begin{tabular}{ccc}
\hline Air flow speed $(\mathrm{m} / \mathrm{s})$ & RPM & Peak emf $(\mathrm{V})$ \\
\hline $\mathbf{4 . 2 3}$ & 115 & 0.654
\end{tabular}


4.95

5.12

5.82

7.11

8.22

9.67

10.23
236

303

504

720

858

986

1154
0.821

0.890

1.136

1.510

1.871

2.252

2.500

\section{Harvester F ('Anti-clockwise' orientation)}

Table 47 Experimental measurements results of energy harvester F in 'anti-clockwise' orientation

\begin{tabular}{ccc}
\hline Air flow speed $(\mathbf{m} / \mathbf{s})$ & RPM & Peak emf $(\mathbf{V})$ \\
\hline $\mathbf{8 . 0 1}$ & 74 & 0.059 \\
$\mathbf{8 . 9 9}$ & 146 & 0.188 \\
$\mathbf{9 . 5 6}$ & 193 & 0.304 \\
$\mathbf{1 0 . 4 9}$ & 267 & 0.556 \\
\hline
\end{tabular}




\section{Harvester G ('Anti-clockwise' orientation)}

Table 48 Experimental measurements results of energy harvester $\mathbf{G}$ in 'anti-clockwise' orientation

\begin{tabular}{ccc}
\hline Air flow speed $(\mathrm{m} / \mathrm{s})$ & RPM & Peak emf $(\mathrm{V})$ \\
\hline $\mathbf{1 0 . 6 8}$ & 206 & 0.395 \\
$\mathbf{1 1 . 3 0}$ & 263 & 0.612 \\
\hline
\end{tabular}

\section{Harvester H ('Anti-clockwise’ orientation)}

Table 49 Experimental measurements results of energy harvester $H$ in 'anti-clockwise' orientation

\begin{tabular}{ccc}
\hline Air flow speed $(\mathbf{m} / \mathbf{s})$ & RPM & Peak emf $(\mathbf{V})$ \\
\hline $\mathbf{8 . 1 6}$ & 124 & 0.124 \\
$\mathbf{9 . 0 1}$ & 151 & 0.204 \\
$\mathbf{9 . 3 8}$ & 178 & 0.271 \\
$\mathbf{1 0 . 3 7}$ & 239 & 0.475 \\
\hline
\end{tabular}

\section{Harvester I ('Anti-clockwise' orientation)}

Table 50 Experimental measurements results of energy harvester I in 'anti-clockwise' orientation
Air flow speed (m/s)
RPM
Peak emf (V) 


\begin{tabular}{lcc}
\hline $\mathbf{5 . 1 6}$ & 100 & 0.083 \\
$\mathbf{5 . 9 5}$ & 433 & 1.16 \\
$\mathbf{6 . 6 2}$ & 516 & 1.56 \\
$\mathbf{7 . 4 0}$ & 620 & 1.99 \\
$\mathbf{8 . 4 1}$ & 748 & 2.56 \\
$\mathbf{8 . 9 9}$ & 794 & 2.78 \\
$\mathbf{9 . 5 8}$ & 870 & 3.04 \\
$\mathbf{1 0 . 2 3}$ & 942 & 3.35 \\
\hline $\mathbf{1 0 . 9 0}$ & 1021 & 3.72 \\
\hline
\end{tabular}

